\title{
Development of a Novel Underground Mine Reconnaissance Robot
}

by

Nick Thompson

\author{
A thesis \\ submitted to the Victoria University of Wellington \\ in fulfilment of the \\ requirements for the degree of \\ Masters \\ in Engineering.
}

Victoria University of Wellington

2019 



\begin{abstract}
Despite advancements in safety technology, underground mining disasters kill hundreds of people each year. Typically after a disaster, a manned response team will enter the hazardous mine to ascertain its condition and rescue any survivors. A robotic entry platform could significantly reduce the risk to the response teams and the time taken to recover any survivors. However, existing mine search and rescue robots have had limited success in past disasters. Two primary aspects caused the failure of the existing platforms; poor rough terrain ability and lack of ingress protection for the harsh mine environment.

HADES, a novel underground mine reconnaissance robot is developed to address these issues. A lightweight yet robust chassis is manufactured from fibreglass. To allow HADES to operate in the potentially explosive atmosphere, the chassis is protected with a positive pressure gas system, designed to meet the A/NZ60079.29 standard. This chassis is sealed against the mine environment with a series of O-rings and lip seals. Whegs are used as the primary locomotion method and are driven with a planetary gearbox and a brushless DC motor. To further improve a rough terrain capability of the locomotion system the rear arm of the chassis is mounted on an actuated pivot, increasing the rough terrain capability of HADES.
\end{abstract}

To ensure the operator can successfully assess and navigate the mine, HADES carries a comprehensive set of environmental and navigation sensors. The internal electronics and locomotion systems are powered with six Li-Po batteries that achieve an operating time of six hours and an expected range of $25 \mathrm{~km}$. 
HADES is $780 \times 800 \times 400 \mathrm{~mm}$ and is mostly sealed to the IP68 standard. The locomotion system is robust and can traverse the majority of the terrain expected in an underground mine. Loss of traction is the only problem encountered with the Wheg design. However, this can be easily fixed by changing the tip shape of the Wheg. 


\section{Acknowledgments}

This thesis would not be possible without the guidance and expertise of several key individuals.

Dale - my supervisor. Your tenacity, humour and legendary attention to detail has allowed me to exceed my own expectations. I am going to miss our weekly meetings and discussions on all things technical.

Tim, Jase and Brandon your technical expertise and ability to deal with my constant requests has been invaluable.

Nick, Alex and Allan your mechanical engineering and production advice helped form many of the key design decisions in this thesis.

Johnathon from Solpont, for the mechanical engineering and fibreglass manufacturing advice.

Hamish, for the countless hours you've put in proofreading, I will be forever indebted. 


\section{Contents}

1 Introduction $\quad 1$

1.1 Thesis Objectives ..................... 2

1.2 Thesis Structure . . . . . . . . . . . . . . . . . 3

2 Background $\quad 5$

2.1 Underground Mines ................. 5

2.1 Terrain ...................... 5

2.1.2 Atmospheric Conditions . . . . . . . . . . . 7

2.2 Other Robotic Platforms . . . . . . . . . . . . . . . . . 9

2.2.1 Non-Deployed Robots . . . . . . . . . . . . . . . . 10

2.2 .2 HADES ........................ 12

2.2 .3 Summary ........................ 16

2.3 Protection Features . . . . . . . . . . . . . . . . 17

2.3 .1 Positive Pressure . . . . . . . . . . . . . . . 18 
2.3.2 Flameproof Enclosures . . . . . . . . . . . . . . . . . . . . . 19

2.3.3 Intrinsic safety $\ldots \ldots \ldots \ldots \ldots \ldots \ldots$

2.3 .4 Summary $\ldots \ldots \ldots \ldots \ldots \ldots \ldots \ldots \ldots$

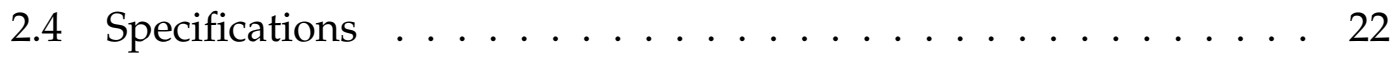

3 Mechanical - Chassis Design 25

3.1 Mechanical Design $\ldots \ldots \ldots \ldots \ldots \ldots \ldots$

3.1 .1 Requirements . . . . . . . . . . . . . . 26

3.1 .2 Implementation $\ldots \ldots \ldots \ldots \ldots \ldots \ldots$

3.1 .3 Motor Mounts . . . . . . . . . . . . . . . . . . . . . . . . 31

$3.1 .4 \quad$ View Ports . . . . . . . . . . . . . . . . . . . . 33

3.1.5 Access Panels . . . . . . . . . . . . . . . . . . . . . . . 35

3.1.6 Battery Mounting . . . . . . . . . . . . . . . . . . 40

3.1.7 Design for Manufacture . . . . . . . . . . . . . . 41

3.2 Rear Arm Pivot . . . . . . . . . . . . . . . . . . 44

3.2.1 Rear Arm Actuation . . . . . . . . . . . . . . . . . . 48

3.3 Positive Pressure Gas System … . . . . . . . . . . . . 50

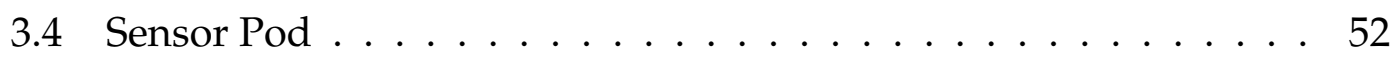

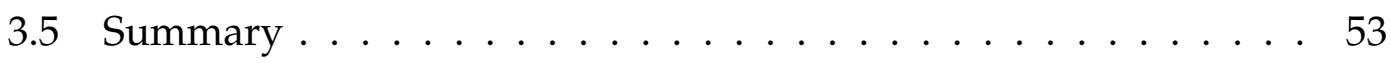


4 Mechanical - Locomotion $\quad 55$

4.1 Wheel Design ......................... 55

4.1 .1 Specifications .................... 55

4.1 .2 Implementation . . . . . . . . . . . . 57

4.1.3 Wheel Design Summary . . . . . . . . . . . . . . . . 60

4.2 Drive ............................... 60

4.2 .1 Gearbox ..................... 61

4.2 .2 Motors ..................... 62

4.3 Wheg and Chassis Support . . . . . . . . . . . . . 64

4.3.1 Revision One ................. 65

4.3 .2 Revision Two . . . . . . . . . . . . . . 68

4.4 Drive System Summary . . . . . . . . . . . . . . . . . 72

$\begin{array}{lll}5 & \text { Electronics - Sensor Systems } & 75\end{array}$

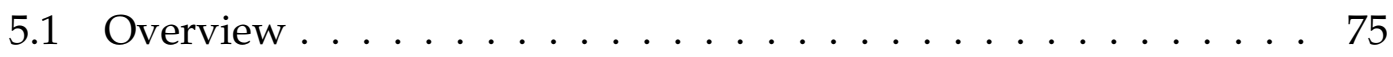

5.2 Environmental Sensors . . . . . . . . . . . . . . 76

5.2 .1 External Sensors . . . . . . . . . . . . . 77

5.2.2 Intrinsic Safety Barrier . . . . . . . . . . . . . . 89

5.2 .3 Internal Sensors . . . . . . . . . . . . . . . 93

5.2.4 Sensor Systems Summary . . . . . . . . . . . . . . . 99 
5.3 Camera Systems . . . . . . . . . . . . . . . . . . . 100

$5.3 .1 \quad$ Requirements . . . . . . . . . . . . . . 100

5.3.2 Implementation . . . . . . . . . . . . . . . . . . 101

5.3.3 Camera Systems Summary ～. . . . . . . . . . . . . 107

6 Electronics - Motor Drive and Power Management 109

6.1 Motor Drive and Control . . . . . . . . . . . . . . . . . . . . 109

6.1 .1 Summary . . . . . . . . . . . . . . . . . . . 114

6.2 Power Supply and Management $\ldots \ldots \ldots$. . . . . . . . 115

$6.2 .1 \quad$ Batteries . . . . . . . . . . . . . . . . . 115

6.2 .2 Battery management . . . . . . . . . . . . . . . 117

6.2.3 Regulated Supplies and Monitoring . . . . . . . . . . 124

6.2 .4 Power PCB . . . . . . . . . . . . . . . . . . . . . . 131

6.2 .5 Summary . . . . . . . . . . . . . . . . 134

7 Software - ROS Control 135

7.1 Robot Operating System . . . . . . . . . . . . . . . 135

7.1.1 Fault Codes . . . . . . . . . . . . . . . . . . 138

7.1.2 Video Controller . . . . . . . . . . . . . . . . . . . . . 139

7.1.3 Motor Control . . . . . . . . . . . . . . . . . . . . . 144 
7.1 .4 Summary . . . . . . . . . . . . . . . . . 148

7.2 Graphical User Interface . . . . . . . . . . . . . . . 148

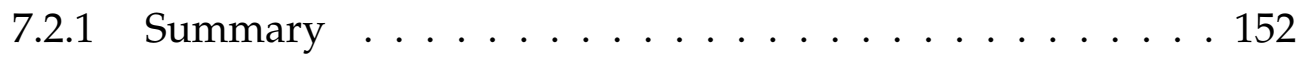

$\begin{array}{lll}8 & \text { Embedded Software } & 153\end{array}$

8.1 Low level Communication . . . . . . . . . . . . . . . . 155

8.2 Main Microcontroller . . . . . . . . . . . . . . . . . . . . . . 159

8.2.1 ROS Integration . . . . . . . . . . . . . . . . 160

8.2.2 Inertial Measurement Unit and Sensor Fusion . . . . . . 162

8.2.3 External and Internal Sensors . . . . . . . . . . . . 168

8.2.4 Positive Pressure Control System . . . . . . . . . . . . . . 172

8.2.5 Watchdog Microcontroller . . . . . . . . . . . . . . 175

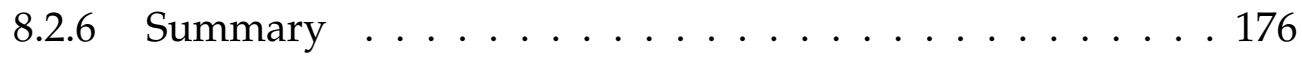

8.3 Power Controller . . . . . . . . . . . . . . . . . . . . 176

8.3.1 Battery Management System _ . . . . . . . . . . 177

8.3.2 Voltage Rail Monitoring . . . . . . . . . . . . . . . . 179

8.3 .3 Summary . . . . . . . . . . . . . . . 180

9 Evaluation and Discussion $\quad 181$

9.1 Locomotion . . . . . . . . . . . . . . . . . . . . . . . . 181 
9.1.1 Compact Dirt/Gravel and Small Boulders . . . . . . . . 182

9.1 .2 Loose Gravel . . . . . . . . . . . . . . . . 183

9.1.3 Large Boulders . . . . . . . . . . . . . . . . . . 185

9.1.4 Reduced Locomotion Power . . . . . . . . . . . . . 186

9.1 .5 Manoeuvrability . . . . . . . . . . . . . 188

9.1.6 Locomotion - Discussion and Summary . . . . . . . . . . 190

9.2 Power Consumption . . . . . . . . . . . . . . . . . 191

9.3 Chassis Evaluation . . . . . . . . . . . . . . . 192

9.3.1 Rear Arm Pivot . . . . . . . . . . . . . . . . . . . . 194

9.3 .2 Ingress Protection . . . . . . . . . . . . . 196

9.3 .3 Thermal Management . . . . . . . . . . . . . . . . 199

9.4 Summary . . . . . . . . . . . . . . . . 201

10 Conclusion and Future Work 203

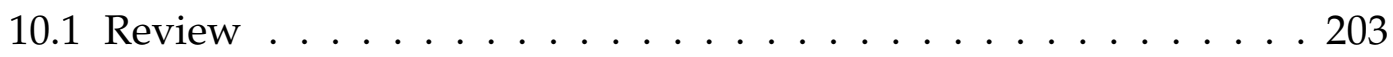

10.2 Future Work . . . . . . . . . . . . . . . . . 205

10.3 Summary . . . . . . . . . . . . . . . . . . . 208 


\section{List of Figures}

2.1 A example layout of an underground mine with a vertical access

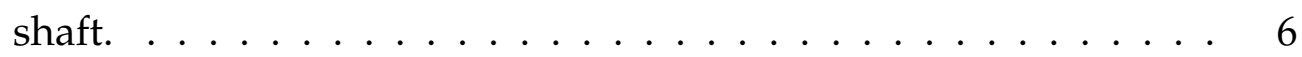

2.2 Underground mines post disaster environment. . . . . . . . . 7

2.3 Wolverine $\mathrm{V} 2$ mine entry robot. . . . . . . . . . . . . . 10

2.4 Defender ROV (left) and Inuktun VGTV (right . . . . . . . . . . . 11

2.5 Images of both Minbot and Numbat illustrating their different locomotion systems. . . . . . . . . . . . . . 14

2.6 HADES revision one climbing over an obstacle. . . . . . . . . . 15

2.7 The prototype node dispensing system modelled next to HADES in their proposed mounting location. [60]. . . . . . . . . . . 16

2.8 The flameproof enclosure used in Minbot-2 . . . . . . . . . . . . . 19

2.9 An example of an intrinsic safety barrier . . . . . . . . . . . . 20

3.1 A comparison between a short and long wheel base for obstacle climbing. . . . . . . . . . . . . . . 27 
3.2 The layer stack up of the fibreglass used in the construction of the HADES chassis. . . . . . . . . . . . . . . . . . . . . . . . . 29

3.3 A CAD view of the chassis internals without the top access hatch or camera systems. . . . . . . . . . . . . . . . 30

3.4 The HADES chassis with all the sub-systems and internal components installed. . . . . . . . . . . . . . 31

3.5 The steel insert used for retaining the mounting bolts for the motor and rear arm pivot sub-assemblies. . . . . . . . . . . . 32

3.6 The motor mount moulded into the fibreglass. . . . . . . . . 33

3.7 The front window and fibreglass mounting bracket. . . . . . . . . 34

3.8 The CAD model of the top access panel assembly without the sensor pod installed. . . . . . . . . . . . . . 37

3.9 The CAD model of the bottom access panel the with the ESCs, power management PCB and the rear arm actuation system. . . . 38

3.10 The rear access Panel without the camera or illumination system installed. . . . . . . . . . . . . . . . . . . . . . 39

3.11 The batteries mounted inside HADES during the field trails. . . . 41

3.12 The mould plug with the aluminium insert for the main chassis fibreglass component. . . . . . . . . . . . . . . . . . . . 42

3.13 The mould plug and mould with a fibreglass part curing in the mould, the indexing tab can be seen on the far side of the moulded part....................... 4 
3.14 An exploded view of the chassis components showing the alignment tabs and motor end caps. . . . . . . . . . . . . 44

3.15 The pivot assembly used in HADES revision one [49]. . . . . . . . 45

3.16 A labelled cross section of the aluminium rear arm pivot. . . . . . 47

3.17 The dissembled rear arm pivot used in HADES. . . . . . . . . . . 48

3.18 The drive unit for the rear arm articulation with the BaneBots P60 182:1 gear box and Justock brushless motor. . . . . . . . . . . . 49

3.19 The internal tank and regulation system for the positive pressure gas system. . . . . . . . . . . . . . . . . 52

3.20 The sensor PCB and sensors mounted to the top plate of the sensor pod. . . . . . . . . . . . . . . . 53

3.21 A 3D printed prototype of the sensor pod without the steel over the sensor protruding out of the top plate. . . . . . . . . . 54

4.1 A four spoke Wheg with $20 \%, 40 \%$ and $60 \%$ duty cycle (left to

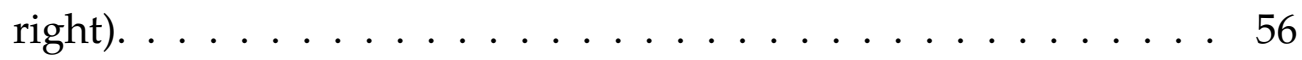

4.2 The Whegs used for the initial trials. . . . . . . . . . . . . . 57

4.3 An exploded view of the five spoke Wheg assembly. . . . . . . . . 59

4.4 HADES moving on loose gravel with the $490 \mathrm{~mm}$ Whegs. . . . . . 60

4.5 The Hobbystar 56112 brushless motor and the Banebots P80 64:1 gear connected together with a bespoke mounting block. . . . . . 63

4.6 The revision one gearbox-bearing block mount. . . . . . . . . . . . 67 
4.7 A cross section view of the revision one bearing block and gearbox support. . . . . . . . . . . . . . . 68

4.8 The disassembled gearbox front end (left) and bearing block (right), showing the alignment boss on the gear box front end. . . . . . . 69

4.9 The revision two gearbox front end, bearing block and shaft. . . . 70

4.10 The complete force diagram for a taper system compressed with a nut $[25] \ldots \ldots \ldots \ldots$. . . . . . . . . . . . . . . . . . . . . . . . .

4.11 The tapered hub mounted to the shaft and drive system. . . . . . 73

4.12 The complete locomotion system when it is not mounted inside HADES. . . . . . . . . . . . . . . . . 74

5.1 The top level block diagram for the electronics system in HADES 76

5.2 The block diagram for the HADES sensor system . . . . . . . . . . 77

5.3 The circuity for the counter, reference and working electrodes of an A4 series gas sensor . . . . . . . . . . . . . . 80

5.4 The circuity for the Auxiliary electrode of an A4 series gas sensor 81

5.5 The support circuity for the ME2-02 oxygen gas sensor. . . . . . . 82

5.6 The support circuity for the 4P-50 combustible gas sensor. . . . . 83

5.7 The circuity for the IRcell-CO2 sensor. . . . . . . . . . . . . . . 84

5.8 The circuity used for measuring the barometric pressure external to the chassis. . . . . . . . . . . . . . . 86 
5.9 The circuity used for measuring temperature and humidity external to the chassis. . . . . . . . . . . . . . 87

5.10 The bottom of the external sensor PCB showing the support circuity, JST and turret connectors . . . . . . . . . . . . 88

5.11 The ground plane cutout around the input pins of an OP296GSZ op-amp. ...................... 89

5.12 The external sensor PCB mounted to a prototype top plate of the external sensor pod. . . . . . . . . . . . . . . 90

5.13 An example of an ia intrinsic saftey barrier . . . . . . . . . . . 90

5.14 The resisitve ignition curves for common gasses found in underground coal mines $[13] \ldots \ldots$. . . . . . . . . . . . . 91

5.15 The layout of an intrinsic safety barrier used in HADES. . . . . . 92

5.16 The schematic implementation of the MPU9250 IMU. . . . . . . . 96

5.17 The circuit for converting the $5 \mathrm{~V}$ signal from the tank pressure sensor to a $3.3 \mathrm{~V}$ signal for the ADC. . . . . . . . . . . . . 97

5.18 The solenoid driving circuitry for the positive pressure control system. . . . . . . . . . . . . . . . 98

5.19 The internal sensing PCB, without the oxygen sensor. . . . . . . 99

5.20 Images of a test scene with a R200 (left) and SR300 (right) Realsense Camera. . . . . . . . . . . . . . . . . . 102

5.21 Depth images of a test scene with a R200 (left) and SR300 (right) Realsense Camera. . . . . . . . . . . . . . . . . . . . . 103

5.22 The front cameras and LEDs mounted inside HADES. . . . . . . . 103 
5.23 Thermal images of a human taken with a Pure Thermal 1 at 5 and $40 \mathrm{~m}$ in a dark hallway. . . . . . . . . . . . . . . . . . . 104

5.24 An image from the side looking fish eye camera. . . . . . . . . . 105

5.25 The Microsoft LifeCam with casing removed . . . . . . . . . . 106

5.26 The schematic implementation of the LDD-700 constant current drivers. . . . . . . . . . . . . . . . . 106

6.1 A comparison of trapezoidal and FOC commutation methods . . 111

6.2 The power and control infrastructure for the connection of one of the VESCs. . . . . . . . . . . . . . . . . . . . . . . 112

6.3 The circuity used to measure the voltage of a single cell. . . . . . . 118

6.4 The temperature measurement circuitry for the battery system in HADES. . . . . . . . . . . . . . . . . 120

6.5 The current sense and switching system for the the motor battery BMS. . . . . . . . . . . . . . . . . . 122

6.6 The two considered power distribution methods for the motor batteries in HADES. . . . . . . . . . . . . . . . . . . . . . . 123

6.7 The Hall Effect sensor used for measuring the current from the control and auxiliary batteries. . . . . . . . . . . . . . . . . 124

6.8 The TLV1117LV33 LDO regulator used for supplying $3.3 \mathrm{~V}$ to each of the sub-systems . . . . . . . . . . . . . . . 125

6.9 The $5 \mathrm{~V}$ regulators and TPS2110A power management switch used for the $5 \mathrm{~V}$ supply rail in HADES. . . . . . . . . . . . . . . 127 
6.10 The resistor divider and hall effect sensor used for monitoring the output from the $5 \mathrm{~V}$ regulators . . . . . . . . . . . 128

6.11 The LM2678S regulator and TPS2011 power management switch used for the $12 \mathrm{~V}$ supply rail. . . . . . . . . . . . . . . . 128

6.12 The high side current switch and hall effect sensor used for monitoring/controlling the power supply of the NUC. . . . . . . . . 130

6.13 The charge inverter and the linear regulator used for the $-5 \mathrm{~V}$ supply.

6.14 The 4.25 V regulator and intrinsic safety barrier for the explosive gas sensor. . . . . . . . . . . . . . . . . . . . 131

6.15 The power management switch for the external sensor pod. . . . 132

6.16 The completed power PCB mounted to the bottom hatch of HADES.132

6.17 The power planes used to route the high current traces for the front left motor and ESC. . . . . . . . . . . . . . . . . . 134

7.1 An example error code call from the video controller that has received no data from the Realsense camera. . . . . . . . . . . . . 139

7.2 A side by side comparison of the raw and corrected images from the side cameras. . . . . . . . . . . . . . . . . . . . . . . 141

7.3 The block diagram for the video system in HADES. . . . . . . . . 144

7.4 The data flow diagram for the hades_locomotion package . . . . . . 146

7.5 The mapping of the different control interfaces to each locomotion control. . . . . . . . . . . . . . . . . . . . 147 
7.6 The GUI presented by the connection_window allowing the operator to connect to the ROS servers and view the real-time log information. . . . . . . . . . . . . . . . . . . . 149

7.7 The GUI interface for the main window class. . . . . . . . . . . . 151

7.8 The configuration settings for updating the motor drive settings. 152

8.1 The communication diagram for the hardware system in HADES. 154

8.2 The state diagram for the embedded software system. . . . . . . . 154

8.3 The data flow diagram for reading and voting the data from the pressure sensors. . . . . . . . . . . . . . . . . 170

8.4 The start-up sequence for the power controller. . . . . . . . . . . . 177

9.1 A plot of motor current vs. battery current for flat ground locomotion. . . . . . . . . . . . . . . . . . . 183

9.2 HADES bottomed out when travelling up a loose gravel slope due to the Whegs digging into the gravel. . . . . . . . . . . . . 184

9.3 HADES travelling over a large bolder field with the two front Whegs not in contact with the ground. . . . . . . . . . . 185

9.4 The battery current supplied to each driven system when travelling at $1.4 \mathrm{~ms}^{-1} \ldots \ldots \ldots \ldots 1$

9.5 The scrapes on the bottom of the HADES chassis from the locomotion tests. . . . . . . . . . . . . . . . . . . 193

9.6 The damage to the running faces of the rear arm pivot assembly. . 194

9.7 A cross section of the steel and bronze rear arm pivot. . . . . . . 196 
9.8 One of the motor mounts with an irregular sealing surface caused by the manufacturing process. . . . . . . . . . . . . . . 197

9.9 HADES floating at the surface for the buoyancy tests. . . . . . . 198

9.10 HADES being held underwater for the submersion testing of the IP68 standard . . . . . . . . . . . . . . . . . . . . 198

9.11 Thermal images of the chassis internals after 30 mins of continuous operation. . . . . . . . . . . . . . 200 


\section{List of Tables}

2.1 A table of common gases in underground mines and their effects [2] 8

2.2 Robotic platforms deployed in underground mining disasters adapted from [17]. . . . . . . . . . . . . . . . . . . . 12

2.3 The summary of pre-existing underground mine rescue robots. . 13

2.4 Protection methods that have been used in previous underground mine exploration robots. . . . . . . . . . . . . . 21

5.1 Target Gasses and their associated sensors. . . . . . . . . . . . 78

5.2 A4 Series sensor load and gain resistors. . . . . . . . . . . . . 81

5.3 Expected ranges and sensor selection for the other external sensors. 85

5.4 Failure mode analysis table for the external pressure sensing system. ........................... 85

5.5 The location and use of the different temperature sensors throughout the HADES chassis . . . . . . . . . . . . . . . . . . . . . . . . . 94

5.6 A comparison between the Microsoft Kinect, SR300 and R200 depth cameras. . . . . . . . . . . . . . . . . . 101 
6.1 A comparison of the different ESCs available for the HobbyStar 56112 BLDC motors. . . . . . . . . . . . . . . . . 111

6.2 The operational limits for the properties of the VESC that trigger a shutdown or soft back-off state. . . . . . . . . . . . . . 113

6.3 Voltage and current requirements for the battery systems on HADES.116

6.4 The safe operating limits for the batteries used in HADES. . . . . 117

6.5 The resistor values used in the divider networks for cell voltage measurement. . . . . . . . . . . . . . . . . . . . . . 119

6.6 A comparison of the two power distribution methods for the motor batteries. . . . . . . . . . . . . . . . . . . 123

6.7 The power requirements for the $5 \mathrm{~V}$ and $12 \mathrm{~V}$ supply Rails. . . . . 126

7.1 The names and functions of the ROS packages used in HADES. . 137

8.1 The standard packet for communication on the I2C bus. . . . . . . 155

8.2 The ROS messages subscribed and published by the main microcontroller . . . . . . . . . . . . . . . . . . . . . 161

8.3 The relationships between the ADC voltages and the sensor target metric . . . . . . . . . . . . . . . . . . . . 170

9.1 Compact gravel and small boulder locomotion success rates. . . . 182

9.2 The success rates of the loose gravel locomotion tests. . . . . . . . 183

9.3 The success / failure table for different scenarios of reduced locomotion testing on flat ground. . . . . . . . . . . . . 187 
9.4 The success / failure table for different scenarios of reduced locomotion testing on sloped ground. . . . . . . . . . . . . 187

9.5 The turning circle test results for HADES on different terrains. . . 188

9.6 The amplitude of the vertical oscillations and the displacement from straight line travel for different travel speeds. . . . . . . . . . 189

9.7 Key system temperatures of HADES after 30 mins of continuous operation. ........................ 200 


\section{Listings}

7.1 The message definition for the motor_ctrl.msg message. . . . . . 137

7.2 The service callback for converting a fault code into a human readable string . . . . . . . . . . . . 138

7.3 The ROS callback for the video_ctrl message. . . . . . . . . . . 141

7.4 The function for setting the compression settings of the theora_image_transport using the parameter server. . . . . . . . . . . . 143

7.5 The ROS callback for an incoming joy message from the X-box controller. . . . . . . . . . . . . . . . . . 145

7.6 The ROS callback for the motor_data message and the function for emitting the data to the main_window class. . . . . . . . . . 150

8.1 The CRC-8 calculation method using the lookup table . . . . . 156

8.2 A snippet of the receivedEvent() method, highlighting the process for setting the BMS relays . . . . . . . . . . . . 157

8.3 The data union between the bat_data struct and an unsigned char array. . . . . . . . . . . . . . . 158 
8.4 A snippet of the ISR that services the fault line in the main microcontroller. . . . . . . . . . . . . . . . 158

8.5 The ROS callback for the motor control data. . . . . . . . . . . . . 161

8.6 The scheduling thread that generates and publishes the IMU data message. . . . . . . . . . . . . . . . . 162

8.7 A snippet of the scheduling thread that reads the IMU and updates the filter values. . . . . . . . . . . . . . . . . 163

8.8 A snippet of the header file for the VESC CAN class. . . . . . . . . 165

8.9 The set RPM method for controlling the target RPM of a VESC. . 166

8.10 The data strucutre that is requested from the VESC . . . . . . . 167

8.11 The start of the InternalSensors class declaration. . . . . . . . . . . 168

8.12 The function for voting on the pressure sensor outputs. . . . . . . 171

8.13 The Zilch thread for controlling the positive pressure regulation system. . . . . . . . . . . . . . . . . . . . 173

8.14 The method used for controlling the activation time of purge solenoid. . . . . . . . . . . . . . . . . . . . . 174

8.15 The public methods of the BMS class used to monitor the batteries in HADES. . . . . . . . . . . . . . . . . . . . . 178

8.16 The header of the $\mathrm{C}++$ class for monitoring the supply rails. . . . 179 


\section{Chapter 1}

\section{Introduction}

Despite recent advancements in safety technology, mining is one of the most dangerous professions in the 21st century. Hundreds of people die in mines every year. A sample of some of the worst mining disasters in the last 20 years include; Pasta de Conchos accident (2006, 65 dead), Upper Big Branch Mine disaster (2010, 31 dead), Copiapo mining accident (2010, 33 trapped), Lily mine disaster (2016, 3 dead) and the motivation for this thesis: The Pike River Mine disaster (2010, 29 dead).

Typically, a manned rescue team responds to an underground mine disaster and is tasked with ascertaining the condition of the mine and rescuing any survivors. By entering the underground mine, the response teams are putting themselves in danger. A robotic vehicle could be used to enter the mine before any manned rescue team, reducing the risk to the response teams. However, historically, the robots that have entered underground mines post-disaster were not well developed to handle the hazardous environment. A new design is required to develop an effective and reliable system that could be deployed to disasters to assess the condition of the mine and search for survivors. The Pike River mine disaster, [57], is an excellent example of how a dedicated mine res- 
cue robot could have expedited the manned entry process had a suitable robotic platform been available.

The Pike River mine disaster occurred on the 19th of December 2010. A methane explosion resulted in 29 deaths and severely damaged the underground infrastructure. Due to the lack of suitable underground mine robots, two New Zealand Defence Force (NZDF) robots designed for bomb disposal were initially deployed. These both failed, one due to water ingress and the other was too large to pass through the damaged workings of the mine. After the NZDF robots failed, two robots from the Australian water industry attempted entry into the mine. These robots were too large and could not pass through the obstructed tunnel. A specialised mine rescue robot was en-route from the United States of America, but rescue operations were terminated before it could arrive. Pike River mine highlighted the need for a local robotic solution dedicated to post-disaster operations in an underground mine.

\subsection{Thesis Objectives}

This project aims to produce a search and rescue robot that can operate safely in the conditions inside a post-disaster mine. This robot would be able to search for survivors and assess the condition of the mine for potential human re-entry. Due to the challenges of the underground mine environment (discussed in Section 2.1), the robot will have to be designed specifically for this purpose. Typically search and rescue robots do not replace manned entry teams, rather they offer an option to enter when it is either unsafe or difficult for a manned entry. The robot can report information on the condition inside the mine and the location of survivors (if there are any). The robot should be able to carry out the following tasks to achieve the goals mentioned above: 
- Traverse debris and rubble.

- Navigate through confined spaces and over uneven terrain.

- Have suitable ingress protection against water, dust and dirt.

- Monitor environmental and atmospheric conditions.

- Meet mine safety standards.

- Operate for extended periods to reach deep into underground mines.

- Self-monitor and arrest potentially dangerous faults.

- Acquire real-time video in dark conditions.

- Communicate with an operator outside the mine.

- Be able to withstand falls and possible tipping.

\subsection{Thesis Structure}

This chapter summarised the motivations for an underground mine entry robot and defined high-level objectives based on previous disasters. Chapter 2 reviews the conditions in an underground mine and summarises pre-existing systems that have been designed to enter underground mines. Chapter 2 concludes with a set of requirements for the robotic platform based on the expected conditions in the mine and existing underground mine entry platforms. Chapter 3 details the design and implementation of a chassis that can safely work in an underground mine environment. The locomotion method and drive systems are developed in Chapter 4. Chapter 5 introduces a comprehensive set of sensors that allow an operator to navigate the robot through the mine and assess the condition of the mine. The motor drive and power delivery systems 
are detailed in Chapter 6. Chapters 7 and 8, cover how the high-level and embedded software systems, respectively, interface with the sensors and actuators of the robot to inform the operator of the robot's status. Chapter 9 evaluates the performance of the completed robotic platform. Chapter 10 summarises and makes recommendations for future advances in the platform. 


\section{Chapter 2}

\section{Background}

\subsection{Underground Mines}

Underground mines are known to be inhospitable places in normal working conditions. After a disaster, when critical infrastructure could be damaged, these conditions will worsen. By evaluating the working conditions of underground mines and the post-disaster conditions of historical disasters, an understanding of expected robot requirements can be gained. A post-disaster underground mine poses two main issues to a robotic entry platform; these are the terrain and the atmospheric conditions.

\subsubsection{Terrain}

An underground mine typically consists of rock wall tunnels that vary between 1.2 and $7.3 \mathrm{~m}$ wide [51]. The tunnels are typically braced with wooden or steel supports that are placed at regular intervals along the tunnels. Depending on the style of the mine there is either a vertical or horizontal access shaft. The 
access shaft leads to the inner workings of the mine. The access tunnel can be up to $2 \mathrm{~km}$ long. Figure 2.1 shows an example layout for a vertical access shaft in an underground mine.

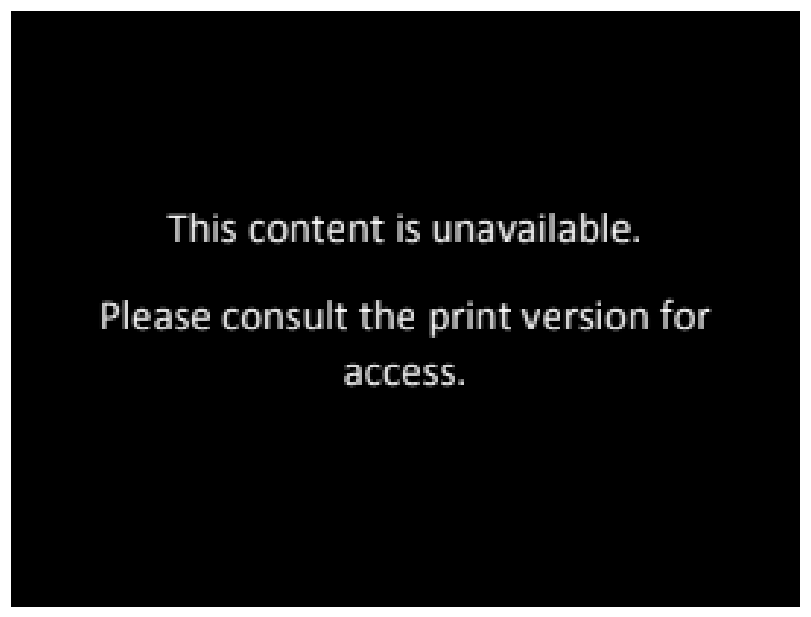

Figure 2.1: A example layout of an underground mine with a vertical access shaft.

The terrain inside an underground mine can be classified into five broad categories; compact dirt/gravel, loose gravel/dirt, train tracks, small boulders (50-200 $\mathrm{mm}$ diameter) and large boulders (>200 mm diameter). Underground mines typically do not contain slopes steeper than $18^{\circ}$ (excluding rubble piles) [51].

Gravel and dirt are usually left on the floor of the mine to allow machinery to drive through the workings. Additionally, to remove the mined ore from the inner workings, train tracks are often run, typically $600 \mathrm{~mm}$ wide and $115 \mathrm{~mm}$ tall [51]. After a disaster, the supports bracing the roof often collapse, leaving the supports or rubble on the floor of the mine. Figure 2.2 shows some images of the inside of various underground mines after a disaster.

A post-disaster mine will often have occluded access due to collapsed tunnel supports/roof and abandoned machinery. Any robotic entry platform will need to be able to climb the expected terrain and move through confined spaces. 

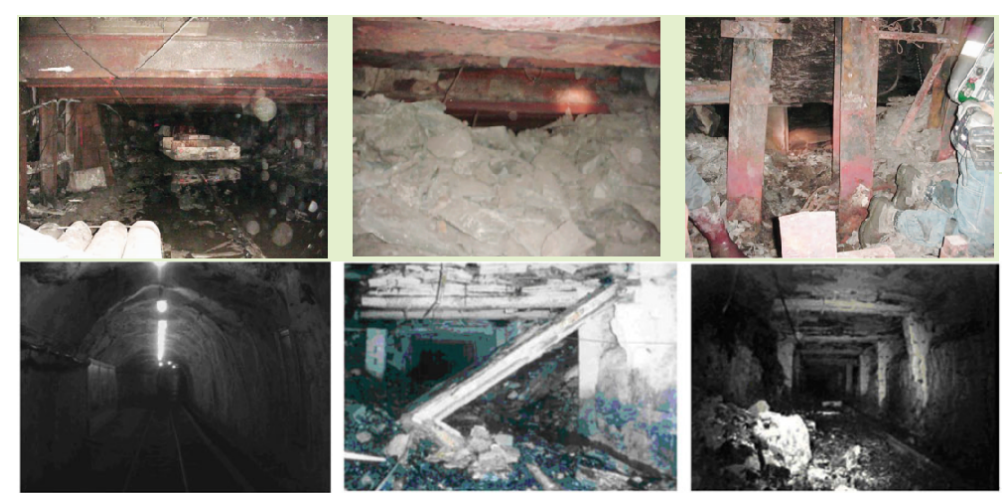

Figure 2.2: Underground mines post disaster environment.

\subsubsection{Atmospheric Conditions}

The atmospheric conditions inside a post-disaster underground mine inform the sensing system that is needed to evaluate and navigate through the mine. The conditions can be assessed by examining historical disasters and government regulations.

After a disaster, the power supply system inside underground mines is typically cut to prevent an electrical fault causing an explosion. This will extinguish any pre-existing lighting systems that are in place within the mine. Therefore the robotic entry platform will have to carry onboard lighting systems.

Underground mines are typically wet places due to the water flowing out of the rock and into the void left by the mine. After a disaster, pumps or other water management system may become damaged, causing the mine to partially or fully flood. The robotic platform will need to be able to operate when surrounded by water and will need to be buoyant.

The New Zealand Government has regulations that control the safe working conditions inside a mine. These cover the maximum allowable concentrations of toxic and explosive gases and air flow requirements [11, 2]. The standards cover eight common gases in underground mines, these are summarised in Ta- 
ble 2.1.

Table 2.1: A table of common gases in underground mines and their effects [2]

\begin{tabular}{|c|c|c|c|}
\hline Gas & Source & Explosivity & Toxicity \\
\hline Nitrogen $\left(\mathrm{N}_{2}\right)$ & $78 \%$ of Atmosphere & No & Oxygen deficiency \\
\hline Oxygen $\left(\mathrm{O}_{2}\right)$ & $20 \%$ of Atmosphere & No & Oxygen deficiency \\
\hline $\begin{array}{l}\text { Carbon } \\
\text { Dioxide }\left(\mathrm{CO}_{2}\right)\end{array}$ & $\begin{array}{l}\text { Burning, rotting, } \\
\text { fuel fumes }\end{array}$ & No & Oxygen deficiency \\
\hline $\begin{array}{l}\text { Carbon } \\
\text { Monoxide (CO) }\end{array}$ & $\begin{array}{l}\text { Burning, rotting, } \\
\text { fuel fumes, blasting }\end{array}$ & $\begin{array}{l}\text { Yes } \\
(12.5-74 \%)\end{array}$ & $\begin{array}{l}\text { High Conc causes: } \\
\text { Vomiting, nausea, } \\
\text { coma and death }\end{array}$ \\
\hline $\begin{array}{l}\text { Hydrogen } \\
\text { Sulfide }\left(\mathrm{H}_{2} \mathrm{~S}\right)\end{array}$ & $\begin{array}{l}\text { Rotting Timbers, } \\
\text { mine water, } \\
\text { rock strata }\end{array}$ & $\begin{array}{l}\text { Yes } \\
(4.5-45 \%)\end{array}$ & $\begin{array}{l}\text { Low Conc: } \\
\text { Irritation of eyes } \\
\text { and throat. } \\
\text { High conc causes } \\
\text { coma and death }\end{array}$ \\
\hline Sulfur Dioxide $\left(\mathrm{SO}_{2}\right)$ & $\begin{array}{l}\text { Fires, some petrolum } \\
\text { based fuels }\end{array}$ & No & $\begin{array}{l}\text { Respiratory } \\
\text { irritation. } \\
\text { Corneal burns }\end{array}$ \\
\hline Methane $\left(\mathrm{CH}_{4}\right)$ & $\begin{array}{l}\text { Coal and rock strata, } \\
\text { rotting timbers }\end{array}$ & $\begin{array}{l}\text { Yes } \\
(5-15 \%)\end{array}$ & Oxygen deficiency \\
\hline Hydrogen $\left(\mathrm{H}_{2}\right)$ & $\begin{array}{l}\text { Coal and rock strata, } \\
\text { rotting timbers }\end{array}$ & Yes $(4.1-74 \%)$ & Oxygen deficiency \\
\hline
\end{tabular}

Methane is the most commonly found explosive gas in underground coal mines as it is produced in the coal-forming process and released when the coal is fractured during mining. Carbon monoxide is also expected in high concentrations after a coal explosion. The robotic entry platform should be able to measure the gases covered in Table 2.1.

An explosion in an underground mine could also cause a fire in the coal 
seam [43]. The temperature and pressure of the atmosphere inside an underground mine will give an indication of air-flow and the presence of fire. If the temperature and the pressure are elevated, then the explosion risk is also elevated as the atmosphere has more energy [71]. In addition to the gas concentrations, the robotic sensor system should measure the pressure and the temperature of the atmosphere.

\subsection{Other Robotic Platforms}

This section provides a brief (due to thesis word count) overview of other robotic platforms. The reader is referred to the references for more detail. However, this section reviews the most salient points of other existing systems to inform the design specifications of the mine inspection robot.

Due to the challenges faced in a post-disaster underground mine, very few dedicated robots have been developed. Even fewer of these robots have been deployed in an actual underground mine disaster. Table 2.2 summarises the deployed robots and their failure modes.

The most commonly deployed robot is Wolverine V2 (Figure 2.3) as it was the only mine certified robot at the time of the disasters [64]. Wolverine V2 is $1.2 \mathrm{~m}$ tall and weighs $500 \mathrm{~kg}$. A substantial portion of this weight is due to the large 7-axis manipulator arm on the front of the robot.

Other deployed robots include Defender ROV [77] and Inuktun VGTV [50]. Defender ROV (Figure 2.4) is large bomb disposal robot $(1.5 \times 0.73 \times 1.2 \mathrm{~m})$, that was adapted for underground mine entry. Like Wolverine V2, Defender ROV has a large manipulator arm that contributes significantly to the size and weight of the robot. Inuktun (Figure 2.4$)$ is small $(0.48 \times 0.3 \times 0.15 \mathrm{~m})$, as it was designed to be lowered through a borehole into the workings of the mine. Both Defender ROV and Inuktun feature a large set of atmospheric and visual 


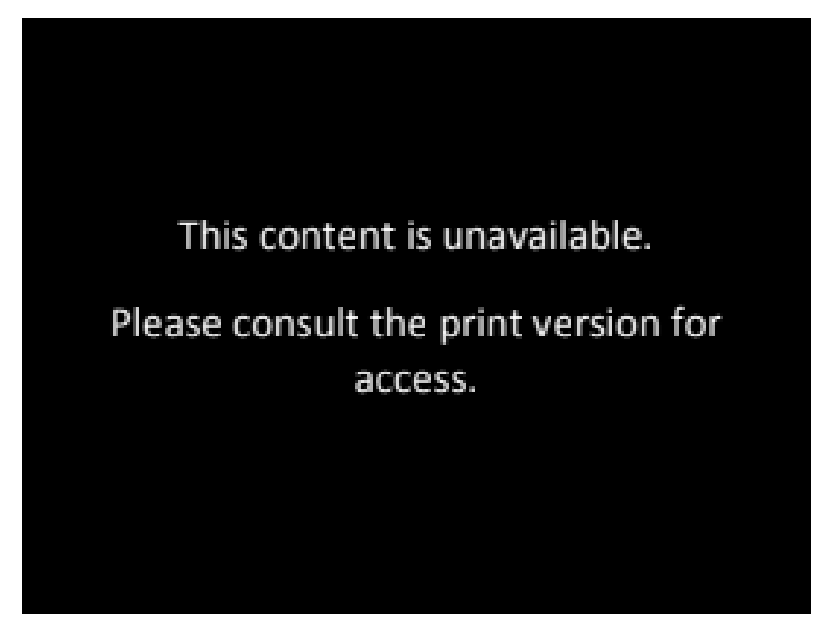

Figure 2.3: Wolverine V2 mine entry robot.

sensors.

The largest failure mode of any of the deployed robots has been the communications tether snapping. The second largest failure mode was because the robotic entry was occluded, prohibiting entry as the robot was too large to pass through the workings of the damaged mine.

\subsubsection{Non-Deployed Robots}

The since the development of Wolverine V2, a number of additional robots have been developed that are specifically designed for underground mine entry. These robots are summarised in Table 2.3.

The robots presented can be categorised into two classes, small form factor and large form factors. Minbot, Lurker 3 and Gemini Scout are small form factor robots, and TeleRescuer, MPI, GMRI, Inspection Robot and Numbat are large form factor robotic platforms. Large form factor robots are typically designed for large mines that do not have occluded access after a disaster. The large form factor designs commonly also carry a large manipulator help clear 


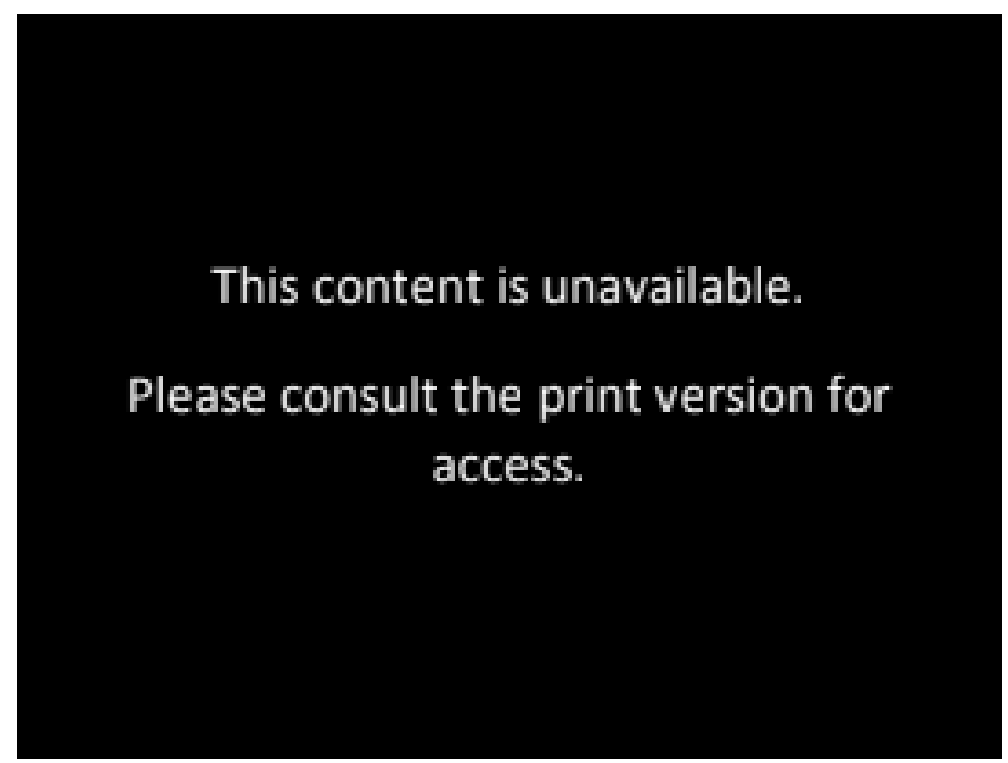

Figure 2.4: Defender ROV (left) and Inuktun VGTV (right

the path of the robot. This increases the size and weight of the robot.

Tracks are the most popular locomotion method for the surveyed underground mine re-entry robots. Tracks commonly need additional degrees of freedom to improve their rough terrain capability. The most common design is the paddle track design, shown on Minbot in Figure 2.5a. The paddles allow the robot to lift the tracks onto an obstacle that a non-paddle track system would not be able to gain traction on.

However, paddle tracks are complex and require many moving parts and drive shafts. Every moving part and drive protrusion through the chassis present another potential failure mode. Reliability issues have been identified with the tracked system on Wolverine-V2 where in one instance the system de-tracked causing the robot to crash mid-rescue.

Wheels are most effective over flat ground. However, they perform poorly when climbing rough terrain. Complex suspension systems are often integrated into wheeled designs to improve their rough terrain ability. The swing arm sus- 
Table 2.2: Robotic platforms deployed in underground mining disasters adapted from [17].

\begin{tabular}{|c|c|c|c|c|}
\hline Year & Mine & Country & Robot & Failure Mode \\
\hline 2001 & Jim Walter No. 5 & USA & Wolverine V2 & \\
\hline 2002 & Barrick Gold Dee & USA & Wolverine V2 & \\
\hline 2004 & Browns Fork & USA & Wolverine V2 & Too tall \\
\hline 2004 & Excel No. 3 & USA & Wolverine V2 & Tether Severed \\
\hline 2005 & DR No. 1 & USA & Wolverine V2 & Tether Severed \\
\hline 2005 & McClane Canyon & USA & Wolverine V2 & Tether Severed \\
\hline 2006 & Sago & USA & Wolverine V2 & Stuck/Crashed \\
\hline 2007 & Midas Gold & USA & $\begin{array}{l}\text { Defender ROV } \\
\text { \& Inuktun }\end{array}$ & \\
\hline 2007 & Crandall Canyon & USA & Inuktun & $\begin{array}{l}\text { Stuck \& } \\
\text { Sensor Fouling }\end{array}$ \\
\hline 2010 & Wangjialing Coal & China & Unknown & \\
\hline 2010 & Upper Big Brach & USA & Wolverine V2 & \\
\hline 2010 & Pike River & $\mathrm{NZ}$ & $\begin{array}{l}\text { NZDF bomb-squad \& } \\
\text { Western Australia } \\
\text { Water Company } \\
\text { robots }\end{array}$ & $\begin{array}{l}\text { Stuck/Short-Circuit, } \\
\text { Stuck/Power loss, } \\
\text { Too large }\end{array}$ \\
\hline
\end{tabular}

pension system used for pairs of wheels on Numbat can be seen in Figure 2.5b.

\subsubsection{HADES}

One innovative, and inexpensive form of a mine inspection robot is HADES. HADES is an underground mine rescue robot developed in 2015 by Molyneaux et al [49]. HADES is a fibreglass robot that is $0.7 \times 0.6 \times 0.1 \mathrm{~m}$ and weighs $20 \mathrm{~kg}$. HADES uses spoked-wheels (or Whegs) as its locomotion method. Whegs aim 
Table 2.3: The summary of pre-existing underground mine rescue robots.

\begin{tabular}{|l|l|l|l|}
\hline Robot & Size & Weight & Locomotion Method \\
\hline TeleRescuer [52, 53] & $2.1 \times 0.74 \times 0.92 \mathrm{~m}$ & $590 \mathrm{~kg}$ & Tracks \\
\hline MPI [38] & $2.4 \times 1.1 \times 1.8 \mathrm{~m}$ & $1100 \mathrm{~kg}$ & Wheels \\
\hline GMRI [40, 39] & & & Wheels \\
\hline Inspection Robot [76] & & & Tracks \\
\hline Numbat [63] & $2.5 \times 1.65 \mathrm{~m}$ & & Wheels \\
\hline Gemini Scout [33] & $0.6 \times 1.2 \times 0.6 \mathrm{~m}$ & $86 \mathrm{~kg}$ & Tracks \\
\hline Minbot [54] & $0.75 \times 1.5 \times 0.59 \mathrm{~m}$ & $130 \mathrm{~kg}$ & Tracks \\
\hline Lurker 3 [67] & & $20 \mathrm{~kg}$ & Tracks \\
\hline
\end{tabular}

to have the rough terrain ability of a legged robot while maintaining the simple, low-cost locomotion system needed for a wheeled system [48]. HADES revision one is shown in Figure 2.6.

The chassis of HADES was manufactured from fibreglass, which is a strong, lightweight material that is formed in a moulding process. The HADES chassis was able to withstand the stresses associated with moving over rough terrain without failing or deforming. Fibreglass is a good thermal insulator, meaning that the internal components could not dissipate thermal energy. This caused heat build-up around the motors, causing them to overheat.

The shape of the chassis kept the centre of gravity low, preventing HADES from rolling over. The spacing between the forward and rear Whegs means that occasionally HADES would bottom out when climbing large obstacles. The rear axle tube pivots relative to the main body of the chassis allowing the rear Whegs to adapt to the terrain.

The chassis was designed to meet the IP68, ingress protection standard. This was achieved using O-rings and lip seals around all the hatches and other subsystem mounts. However, during testing, the chassis did not meet this stan- 


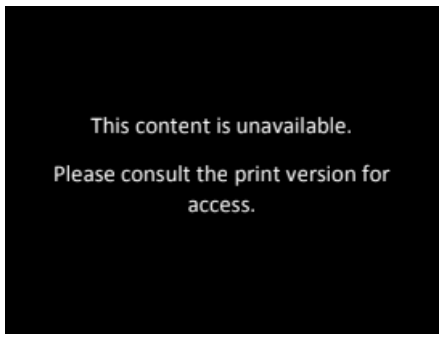

(a) Minbot climbing over an obstacle with the paddle track design.

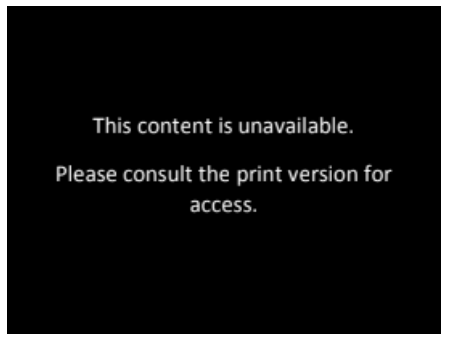

(b) Numbat showing the swing arm system on each wheel.

Figure 2.5: Images of both Minbot and Numbat illustrating their different locomotion systems.

dard. The chassis leaked when submerged, but was able to remain watertight when exposed to high-pressure water jets.

\subsubsection{HADES Communication}

Due to the complexity of the terrain inside an underground mine, HADES was designed to be teleoperated. As identified in Section 2.2, tethers are the primary cause of failure for robotic entry systems. To allow the transmission of video and robot telemetry wireless commutation system is often preferentially used.

The rock surrounding an underground mine will block any wireless signal that has the bandwidth required to transmit video for teleoperation. A repeater node system is proposed in the HADES system to allow wireless communication to function in an underground mine. Two prototype repeater designs have been developed, one using 2.4 GHz and one using $900 \mathrm{MHz}$ [29, 78]. The $900 \mathrm{MHz}$ system is preferred as the lower frequency means the signal propagates further inside an underground mine, although this is achieved at the cost of lower operating bandwidth. 


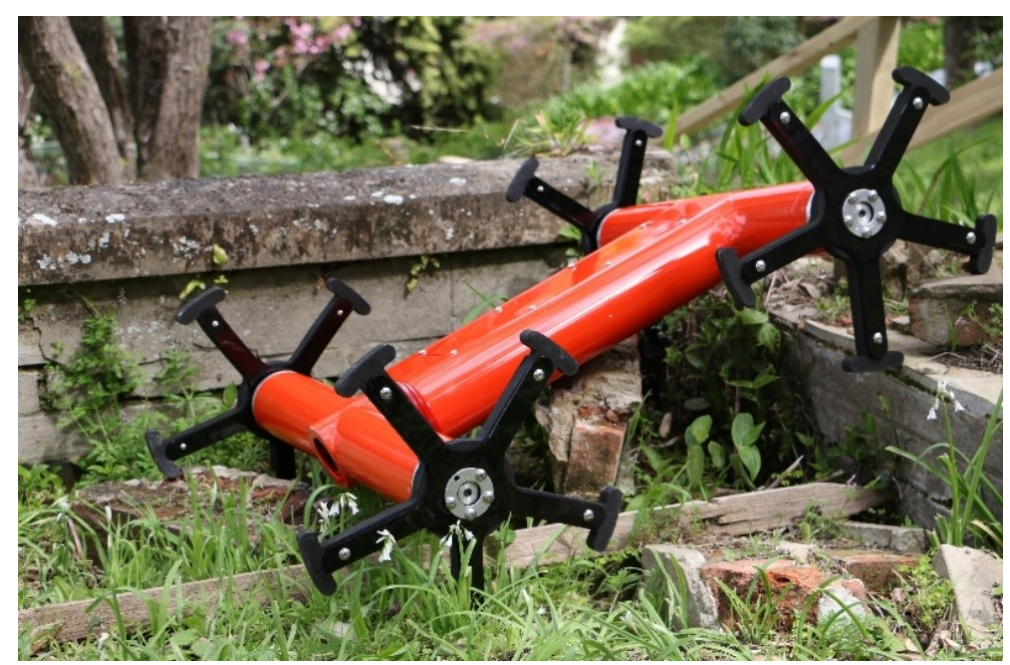

Figure 2.6: HADES revision one climbing over an obstacle.

The typical line of sight range of each repeater is $150 \mathrm{~m}$. To reach the head of an underground mine like Pike River up to 30 nodes could be needed. HADES will need to carry these and drop them at critical points throughout the mine. Two prototype solutions have been developed for carrying and dispensing the communication nodes.

The first of these uses leadscrews and guide rails to hold the nodes on HADES. When the leadscrews are turned, the nodes are pushed off the guides and fall to the ground. The system was designed to mount onto the side of HADES and could carry up to 20 nodes. This design relied on the nodes landing with the correct antenna orientation to function.

To minimise the risk of dropping the nodes in an incorrect orientation, a node design that self-rights was subsequently developed [60]. The node has folding arms that slowly release once the node has been dispensed, forcing the node into the correct orientation. The node dispenser was also updated to reflect the changes in the node design and can carry up to 36 nodes. Figure 2.7 shows a CAD model of the prototype node design models next to HADES. 


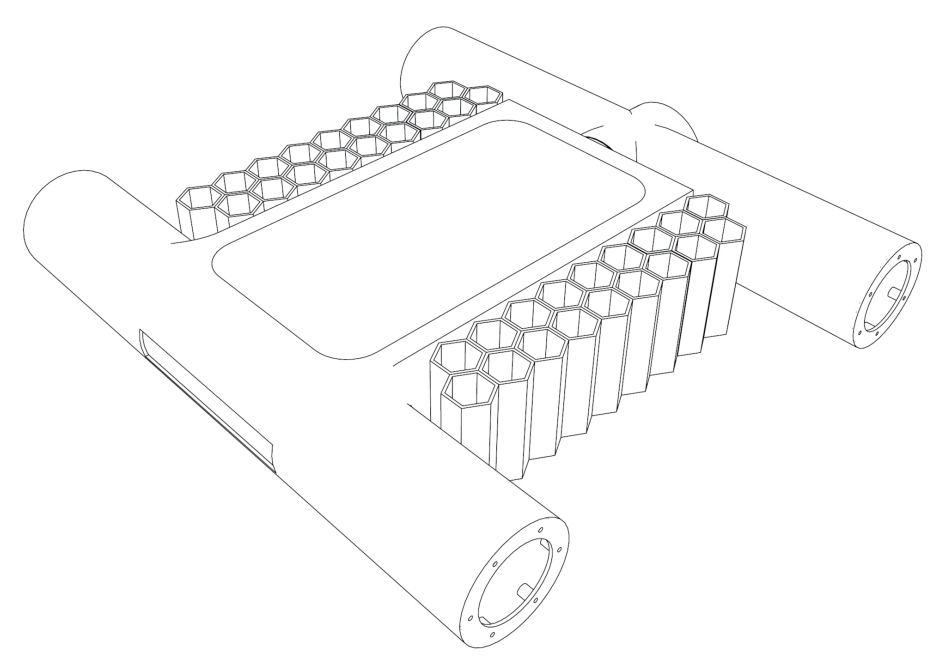

Figure 2.7: The prototype node dispensing system modelled next to HADES in their proposed mounting location. [60].

\subsubsection{Summary}

Large form factor robots are hard to deploy and limited in their ability to navigate through an underground mine. This is due to their size and weight. Small form factor robots are therefore much better suited to deployment into a remote mine, especially one that might have partially occluded access due to damage from an explosion.

Tracks and wheels are the two most common locomotion methods for robotic recuse platforms. However, tracks are often expensive system components as there are many moving parts. Wheels are simple and easy to obtain. However, they often need to be combined with a sophisticated suspension system to be able to traverse the complex terrain found in underground mines. HADES presented an alternative that has neither the cost of tracks or the poor locomotion ability of wheels. Spoked-wheels only require a single part, per spoked-wheel, to be in contact with the ground and are cheap to produce. 


\subsection{Protection Features}

As an underground mine can contain potentially explosive levels of methane and other flammable, gases, any device inside the mine has to comply with strict safety regulations (Section 2.1.2). In New Zealand, these regulations are set out in the A/NZ 60079.X standards, which are drawn from standards set by the International Electrotechnical Commission (IEC) [37]. The IEC standards dictate the minimum protection levels needed, but it is generally accepted that these specifications should be exceeded [2].

There are numerous protection levels outlined in the 60079.X standards [11, 12]. There are three protection classes I, II and III for different ignition sources: gas, dust and fibre respectively. These are broken down into groups A, B and C based on the energy needed for ignition of the explosive material. Finally, there are two different divisions based on the likelihood of explosive levels being present around the device. Div 1 for normal operating conditions and Div 2 for abnormal conditions.

While the standards describe a large number of approaches for managing explosive atmospheres, only a few are typically applied to robotic platforms. These approaches are flameproof enclosures, positive pressure enclosures and intrinsic safety barriers. Flameproof enclosures do not prevent a fire/explosion. Instead, they contain the energy and release it over time through small flame paths. A positive pressure enclosure houses the electronics in an inert atmosphere that is at a higher pressure than the surrounding atmosphere. This prevents explosive gasses reaching the internal electronics and extinguishes any internal fires immediately. An intrinsic safety barrier is an electronic device that sits between the protected electronics and any electronics contacting the explosive atmosphere. The intrinsic safety barrier prevents current and voltage conditions that could cause an ignition from reaching the atmosphere. 


\subsubsection{Positive Pressure}

Keeping an enclosure at a positive pressure prevents explosive gases from contacting internal ignition source(s) as the explosive gases are pushed out of the enclosure by the higher pressure. Ensuring that the internal atmosphere inside the enclosure is inert prevents a fire from starting inside the enclosure.

Positive pressure systems can be broken down into two approaches: freeflowing and static. A free-flowing positive pressure relies on a constant external airflow to maintain positive pressure whereas the static approach maintains a constantly sealed and pressurised vessel, with minimal or no gasflow.

The constant gasflow method is commonly used to protect large industrial equipment (electrical generators) from explosive atmospheres. The static approach is the most common with robotic platforms as a constant gas source is not needed. A static positive pressure system must keep the enclosure at $50 \mathrm{~Pa}$ higher pressure than the external atmosphere to ensure that the external atmosphere does not enter the enclosure. To be considered inert, the internal atmosphere must also be less than $1 \%$ oxygen and not contain any explosive gasses.

Five of the robotic platforms discussed in section 2.2 implement a positive pressure explosion prevention system: these are Lurker-3, Numbat, the Inspection Robot, MPI and HADES. All the systems use nitrogen as the positive pressure gas. Lurker-3, MPI and HADES maintain a positive pressure differential with an onboard pressure system, whereas the Inspection Robot is filled before entry and relies on the sealed enclosure to maintain the pressure differential. The literature for the Numbat robot states that the enclosure is nitrogen flooded, but does not discuss the mechanism or other details. 


\subsubsection{Flameproof Enclosures}

Unlike a positive pressure system, flameproof enclosures allow the contents to circulate air with the external atmosphere. However, the path between the external atmosphere and internal electronics is long, has sharp turns and limited width. This arrests any flames or explosion, preventing the external atmosphere from igniting.

Five of the previously discussed robotic platforms use flame-proof enclosures; these are Wolverine v2, Defender ROV, Gemini Scout, MPI, Minbot and Telerescuer chassis design. CMDR, Minbot and the rocker-type chassis all use flat seals to seal the entire chassis to prevent the explosive energy escaping, whereas Wolverine V2 only seals the motor enclosures. Figure 2.8 shows the sealing surfaces used in Minbot-2.

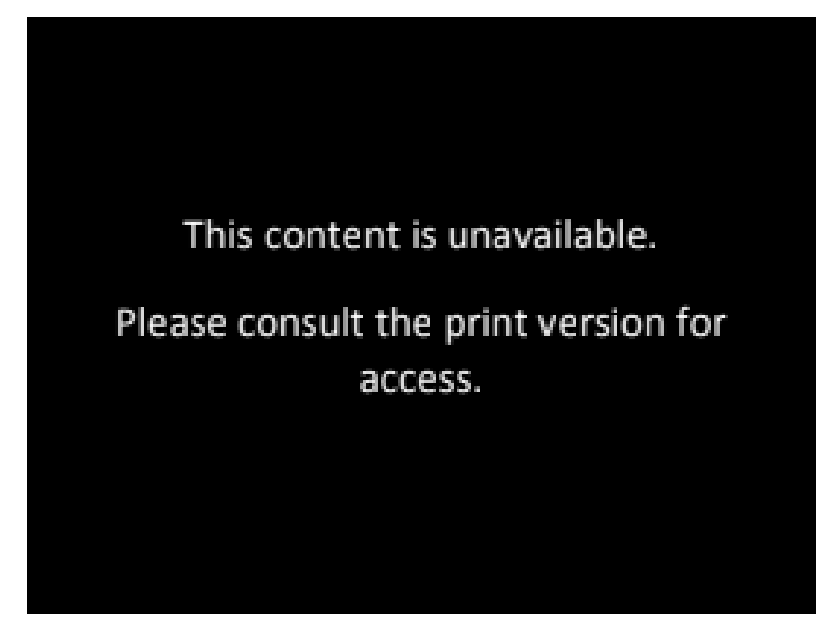

Figure 2.8: The flameproof enclosure used in Minbot-2.

The IEC standards state that any electronics contained in a flameproof enclosure need to be de-energised when the gas concentrations surrounding the enclosure become explosive. Additionally, the materials needed to satisfy the tensile strength requirement for a flameproof enclosure will increase the weight of the robotic chassis significantly. This makes flame-proof enclosures imprac- 
tical for a lightweight rescue robot that needs to operate in a post-disaster underground mine.

\subsubsection{Intrinsic safety}

Intrinsic safety is needed for any electronics that are not protected by the previously discussed protection methods. Any component or circuit that is exposed to the explosive atmosphere and is capable of dissipating enough energy to ignite the atmosphere must be protected with an intrinsic safety barrier. The barrier consists of power resistors, Zenner diodes and fuses to limit the voltage and currents in the external circuit. The 60079.11 standard dictates the maximum currents and voltages allowed for different applications and protection levels which then governs component choice. Figure 2.9 shows an example intrinsic safety barrier.

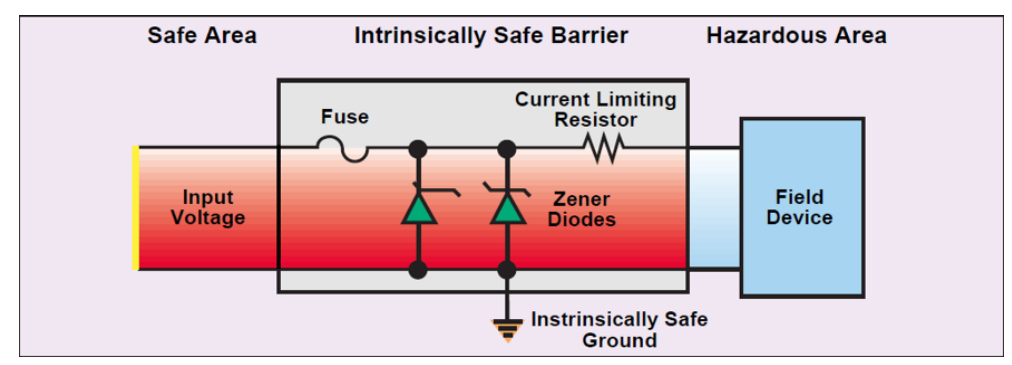

Figure 2.9: An example of an intrinsic safety barrier

HADES and MPI are the only two platforms to report the use of intrinsic safety barriers, none of the other platforms previously discussed document such use. 


\subsubsection{Summary}

Explosive atmosphere certification is critical for protecting the robotic platform and any people operating around it. Components inside the robots chassis can either be protected by a flame-proof enclosure or a positive pressure gas system. Electronics that are exposed to the atmosphere need to be protected by an intrinsic safety barrier. Gaining explosive atmosphere certification is complicated and time-consuming, so few robotic platforms obtain official certification. From the robotic platforms surveyed in Section 2.2, ten have implemented explosion protection measures, these are summarised in Table 2.4.

Table 2.4: Protection methods that have been used in previous underground mine exploration robots.

\begin{tabular}{|l|l|}
\hline Robot & Protection Features \\
\hline Wolverine V2 & Flameproof \\
\hline Inktun & - \\
\hline Defnder ROV & Flameproof \\
\hline TeleRescuer & Flameproof \\
\hline MPI & Flameproof, Intrinsic Safety and Positive Pressure \\
\hline GMRI & - \\
\hline Inspection Robot & Positive Pressure \\
\hline Numbat & Positive Pressure \\
\hline Gemini Scout & Flameproof \\
\hline Minbot & Flameproof \\
\hline Lurker 3 & Positive Pressure \\
\hline HADES & Intrinsic Safety Barriers, Positive Pressure \\
\hline
\end{tabular}




\subsection{Specifications}

HADES represents a significant advancement in the field of underground mine robots. Due to the similarity between the chassis shapes and locomotion methods, the new robotic platform will be named HADES. To disambiguate, the initial HADES will be referred to as HADES V1 for the remainder of this thesis. To work within the confines of the underground mine, HADES cannot be larger than $1 \times 1 \times 0.6 \mathrm{~m}$. HADES must be lightweight to allow it to easily and quickly be transported to remote disaster sites. To prevent damage to the internal components, the chassis temperature should be maintained below $50{ }^{\circ} \mathrm{C}$.

As discussed in Section 2.1, underground mines are muddy wet environments. HADES should be buoyant so that it can pass through flooded areas of the mine without sinking. To be able to move through flooded areas and prevent water, dust or mud ingress, the robot should meet the IP68 waterproofing standard.

HADES will continue the use of Whegs as they have demonstrated that they are capable over differing terrain types and are significantly cheaper and simpler than tracked systems. The drive system will need to enable HADES to keep up with a human walking pace at $1.4 \mathrm{~ms}^{-1}$. HADES should have an operating time that allows it to explore pike river mine extensively. This involves travelling down the $4 \mathrm{~km}$ access tunnel, exploring the workings and then exiting the mine. When travelling at $1.4 \mathrm{~ms}^{-1}$, HADES will need to be able to operate for a minimum of four hours.

A battery system is required to supply power to the drive motors and control electronics. The battery should be able to provide the required currents and voltages to the system components. Additionally, the battery system should monitor the state of the batteries and protect them from unsafe conditions.

HADES will need to operate in an atmosphere that contains explosive levels 
of gas, and so will need to be designed to meet the Class 1 Division 1 explosive atmosphere standards. As flameproof enclosures are large and heavy, HADES will use a positive pressure explosion prevention system to protect the internal electronics. Intrinsic safety barriers will be implemented to safeguard any sensors or other electronics that are outside the chassis.

HADES will carry a comprehensive set of sensors that help the operator navigate the underground mine and inform rescue efforts. The sensor system will sample the eight most common gasses in an underground mine as well as the external pressure and temperature. To allow the operator to navigate HADES though the mine, a set of cameras will provide visual feedback. As the mine will be extremely dark after a disaster, an internal lighting system is needed.

The operation of HADES will be primarily completed with an 802.11 wireless network for prototyping. Integrating with the communication system eventually once it has matured. To integrate the communication system, HADES will need a mounting point for the node dispensers. The software system will also need to carefully manage bandwidth utilisation for the control and telemetry data to prevent overuse of the communication system. 


\section{Chapter 3}

\section{Mechanical - Chassis Design}

A post-disaster underground mine will contain many challenges for robotic exploration. For example, the mine could have falling debris or partial collapses that obstruct the access tunnel. Additionally, underground mines are often wet environments and, after a disaster event, can be fully or partially flooded.

The chassis of HADES needs to house and protect the electronics, sensors and drive systems while traversing through a post-disaster mine. Therefore, the chassis will need to be able to withstand impacts from falling debris, fall short distances and be water-tight. The underground mine may also contain explosive gases. Therefore the chassis will need to comply with the explosive atmosphere series of safety standards covered in Section 2.3. 


\subsection{Mechanical Design}

\subsubsection{Requirements}

The chassis needs to be able to be able to manoeuvre in the confines of an underground mine reliably. To achieve this, HADES will need to be small enough to enter and turn in confined spaces. Molyneaux et al. identified $1000 \times 1000 \mathrm{~mm}$ as a maximum size for HADES revision one, this specification is continued for HADES revision two [49]. HADES will also need a low centre of gravity to remain stable on steep and uneven terrain.

During the testing of HADES revision one, it was noted that HADES would bottom out when climbing over large obstacles. This was due to the long distance between the forward and rear axles (wheelbase). By decreasing this distance relative to the wheel diameter, the risk of bottoming out can be reduced. Figure 3.1 shows the difference between short and long wheelbases when climbing an obstacle. HADES revision one has a wheel diameter to wheelbase ratio of 0.63 , to decrease the chances of bottoming out, this ratio needs to be increased.

The chassis should be able to adapt to terrain features to increase the rough terrain capability of HADES. In revision one of HADES, this was achieved by allowing the rear arm of the chassis to pivot relative to the main chassis. The pivot proved effective for HADES revision one, a second iteration of the pivot should be implemented for HADES revision two [49].

The chassis will need mounting points for the internal and external electronics, sensors and drive systems. The mounting points for the motors and other high load sub-systems will need to be reinforced due to the varying terrain that HADES will need to overcome. The chassis will also need to carry the communication node dispenser systems, discussed in Section 2.2.2. These will mount 


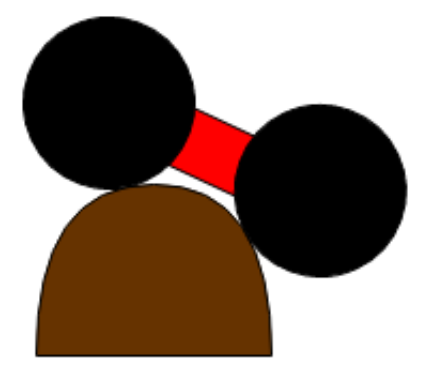

(a) A short wheelbase successfully maintaining traction over an obstacle.

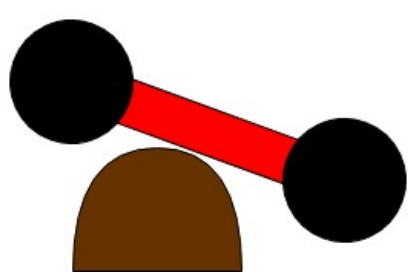

(b) A long wheelbase bottoming out over an obstacle.

Figure 3.1: A comparison between a short and long wheel base for obstacle climbing.

to the outside of the chassis and will need to access power and signals from the internal control electronics. As the finished form of the dispenser systems is not yet fully known, there should be a generic mounting system on HADES that can be modified once the form of the dispenser systems is finalised.

To protect the internal electronics from water damage, HADES will need to seal to prevent water and particulate matter ingress into the chassis. The International Protection Marking, IP code, is a common method for defining the degree of protection an enclosure provides. Ideally, HADES should seal to IP standard, IP68. An IP68 rating means that HADES should be able to seal against all particulate matter ingress and seal against water ingress up to $1 \mathrm{~m}$ of submersion depth.

The camera systems will need view ports to see the environment surrounding the chassis. The view ports will need to be optically and thermally clear and made from a tough, scratch resistant material. Additionally, the external gas sensor systems will require a mounting point that is protected from physical damage but has good air flow for the sensors. 


\subsubsection{Implementation}

\subsubsection{Material Selection}

When climbing rough terrain, the chassis will be put under high torsional loads. The rough terrain could also collide with and scrape along the chassis. The chassis material must, therefore, be able to withstand these torsional and impact loads. Additionally, the chassis needs to be lightweight to reduce the locomotion power required. Keeping the weight of the chassis low also enables easier deployment as mines are often in remote locations that could need HADES to be transported some distance before deployment.

Molyneaux et al. identified fibreglass as a suitable material for HADES revision one [49] having compared it to aluminium, stainless steel, titanium and carbon fibre. Fibreglass was selected because it has the highest specific strength to cost ratio from the examined materials. Fibreglass can also be easily manufactured into complex shapes. It can be strengthened in key areas by adding additional fibres or metal inserts to the resin at manufacture.

Fibreglass material is made from glass fibres impregnated in an epoxy resin. The orientation and number of fibres determine the axis and magnitude of the fibreglass' strength. As advanced finite element analysis to determine ideal fibre orientation and type for the chassis is outside the scope of this project, HADES is constructed with a "cover all" fibre layer stack up. The fibre used in HADES consists of four layers of continuous fibre laid at $0^{\circ}, 90^{\circ}, 45^{\circ}$ and $-45^{\circ}$ and two layers of $50 \mathrm{~mm}$ Chopped Strand Mat (CSM). The two layers of CSM are laid between the layers of continuous fibre. On the outside of the stack up there is a layer of gel-coat to provide a smooth but tough surface finish. Figure 3.2 shows the layer stack up for the fibreglass used in HADES.

The fibreglass is hand laid in a mould and resin is impregnated through all the layers. Vacuum forming is not used to reduce cost. This results in a non- 


\begin{tabular}{|c|}
\hline CSM \\
\hline-45 deg continuous \\
\hline 45 deg continuous \\
\hline CSM \\
\hline 90 deg continuous \\
\hline 0 deg continuous \\
\hline Gel-coat \\
\hline
\end{tabular}

Figure 3.2: The layer stack up of the fibreglass used in the construction of the HADES chassis.

uniform fibreglass sheet that varies between $2 \mathrm{~mm}$ and $5 \mathrm{~mm}$ thick. Where the design tolerances are critical, care will have to be taken in the manufacture of the chassis to ensure that the variance in sheet thickness is minimised. The design considerations and implementation of the moulds and moulding process are covered in Section 3.1.7.

\subsubsection{Design}

As stated in Section 3.1.1, the chassis cannot be larger than $1000 \times 1000 \mathrm{~mm}$ to manoeuvre within the confines of the underground mine. The internal components were selected to fit within a small chassis. Once the internal components had been selected, the chassis was designed to house them with minimum free space. Figure 3.3 shows a CAD model of the chassis design with the internal components.

As mentioned, the wheel-diameter to wheelbase ratio needs to be increased to minimise the likelihood of bottoming out. The wheels used in HADES are $570 \mathrm{~mm}$ in diameter (Section 4.1), thus to increase the ratio, the wheelbase needs to be less than $890 \mathrm{~mm}$. The shortest achievable wheelbase of the chassis, given the space required for the internal components, is $640 \mathrm{~mm}$, this increases the 


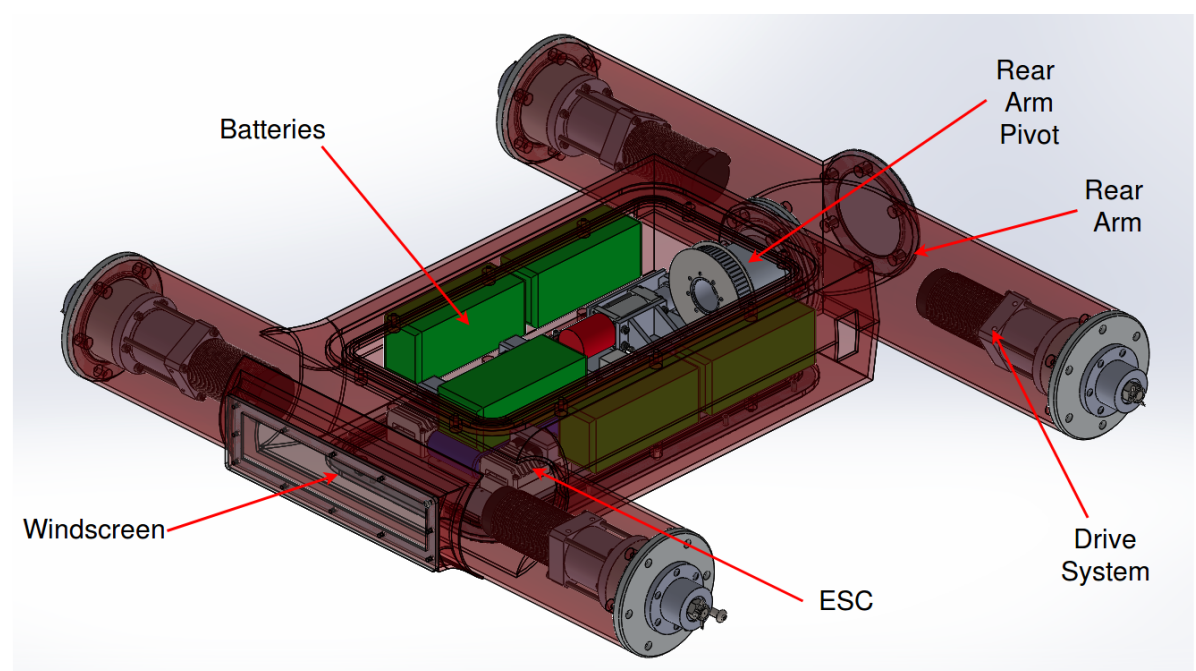

Figure 3.3: A CAD view of the chassis internals without the top access hatch or camera systems.

ratio to 0.89 .

To mount the communication dispenser systems to the outside of the chassis, the sides of the main body are flat surfaces. This allows bolt holes and other cutouts to be added when the dispensing system is finished. Like the main hatch (Section 3.1.4) and motor mounts (Section 3.1.3), threaded inserts can be bonded into the chassis for secure mounting.

The chassis will be manufactured from moulded fibreglass. This process requires that the chassis is made from two half-shell designs that are identical. To ensure the finished parts can be released from the moulds, a minimum draft angle of $3^{\circ}$ on all surfaces is required. To ensure that the draft angle is correct, SolidWorks was used to analyse and rectify the chassis design.

The resultant chassis is $780 \times 800 \times 140 \mathrm{~mm}(\mathrm{~L} \times \mathrm{W} \times \mathrm{H})$, and weighs an estimated $30 \mathrm{~kg}$. The requirements state that the chassis must be buoyant (Section 3.1.1). For this to be true, the density of the chassis must be less than the density of water $\left(1000 \mathrm{kgm}^{-3}\right)$. 
Using SolidWorks to analyse the chassis design, the displacement volume is $0.048 \mathrm{~m}^{3}$ and the mass is $30 \mathrm{~kg}$. This results in a density of $641 \mathrm{kgm}^{-3}$ which is significantly less than the density of water. Therefore, the chassis will float. Figure 3.4 shows the chassis with all the sub-systems installed.

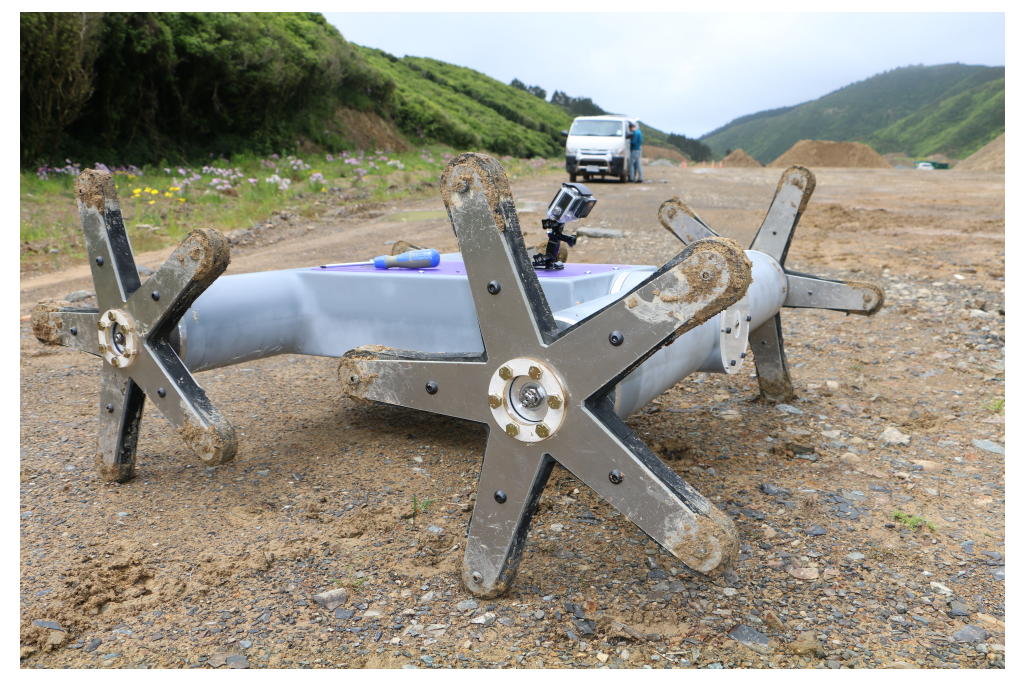

Figure 3.4: The HADES chassis with all the sub-systems and internal components installed.

\subsubsection{Motor Mounts}

The chassis needs to mount to the rear arm pivot (Section 3.2) and the drive systems (Section 4.2). The points at which these systems mount to the fibreglass chassis will be subjected to large loads in normal operation. As the fibreglass is not strong enough to withstand these loads, the chassis needs to be reinforced around these mounting points. The mounting points should all be identical to minimise the engineering effort and production cost. They will also need to seal to meet the IP68 standard.

The drive systems and rear arm pivot need to be removable from the chassis to allow for maintenance. Six M5 bolts are used to allow for this. These 
are placed in a circular pattern with a Pitch Circle Diameter (PCD) of $114 \mathrm{~mm}$. These bolts will be transferring all the load from the sub-assemblies to the main chassis. The largest calculated load on a sub-assembly is from the rear arm pivot which needs to support torques of up to $147 \mathrm{Nm}$ (Section 3.2). Equation 3.1 gives the maximum torsional strength $\left(\tau_{\max }\right)$ of a joint clamped with a circular pattern of bolts (ignoring the frictional component of the joint strength).

$$
\tau_{\text {max }}=A_{n} R_{m} r_{p c d} n,
$$

where $R_{m}$ is the minimum tensile strength of the bolt, $A_{n}$ is the nominal root area of the bolt, $r_{p c d}$ is the radius of the bolt hole pattern, and $n$ is the number of bolts in the pattern. The nominal root area $\left(A_{n}\right)$ of a grade $8.8 \mathrm{M} 5$ bolt is $14.2 \mathrm{~mm}^{2}$, and the minimum tensile strength is $800 \mathrm{MPa}$. For a six hole pattern of M5 bolts on a PCD of $114 \mathrm{~mm}$, the maximum shear torque is $3885 \mathrm{Nm}$. When derated by a safety factor 0.6 (as per [70]) the maximum torsional strength is $2331 \mathrm{Nm}$, which is higher than the $147 \mathrm{Nm}$ expected maximum load.

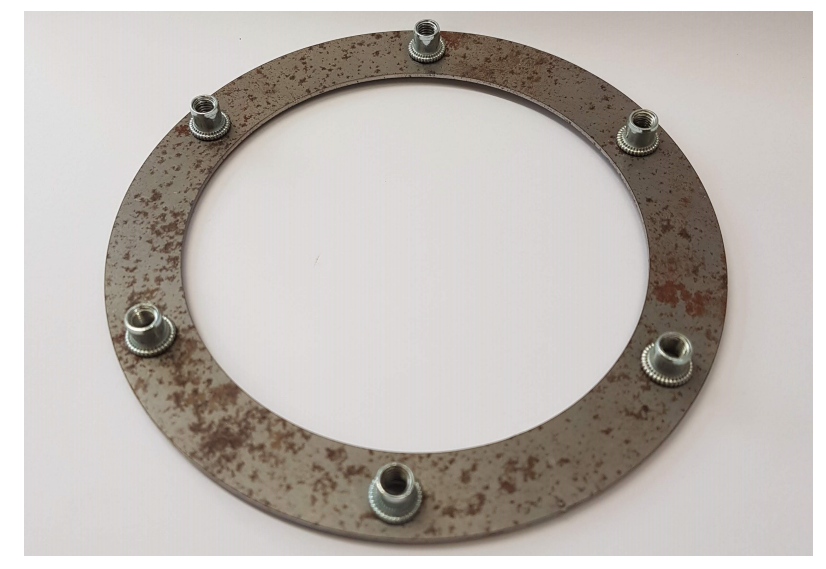

Figure 3.5: The steel insert used for retaining the mounting bolts for the motor and rear arm pivot sub-assemblies.

The fibreglass needs reinforcing to be able to withstand the loads from the six bolts, and the bolts also need to mount into threaded holes. A $1.2 \mathrm{~mm}$ laser 
cut steel insert is used to strengthen the fibreglass which also has six M5 rivnuts to receive the mounting bolts. The steel insert is bonded into the fibreglass at manufacture and acts to distribute the torsional and radial loads from the bolts throughout the fibreglass. The steel insert can be seen in Figure 3.5.

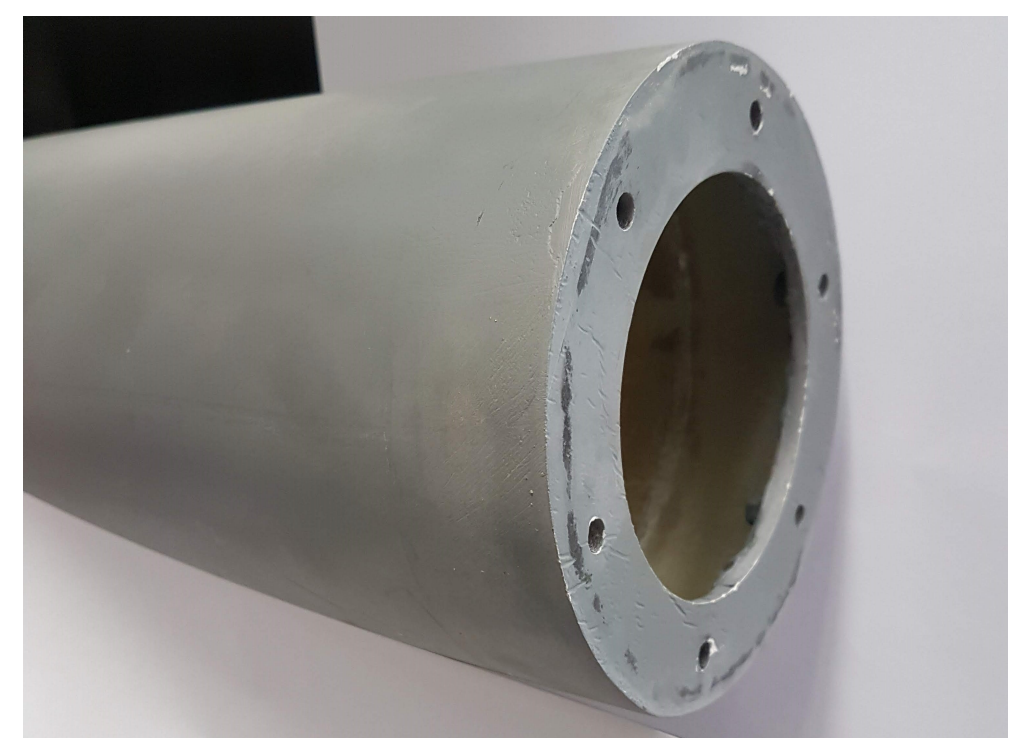

Figure 3.6: The motor mount moulded into the fibreglass.

To seal the bolts holes to prevent water and dust ingress, the fibreglass is bonded over the rear of the bolt holes. To allow for the drive and rear arm pivot system to mount to the chassis, a $90 \mathrm{~mm}$ hole is cut in the fibreglass. Figure 3.6 shows the mounting feature on the fibreglass.

\subsubsection{View Ports}

The camera system in HADES needs view ports so it can see the environment surrounding the chassis. Four view ports are required, the forward and side looking view ports are bonded directly to the chassis, the rear looking view port is integral to the rear access panel (Section 3.1.5.2). The view ports need to be optically transparent to maximise image clarity and to minimise internal 
reflections from the illumination LEDs. The forward looking view port material also needs to be able to pass emissions from the infrared spectrum for the thermal camera.

The most common material for thermal camera windows is calcium fluoride glass. However, calcium fluoride glass is not robust and is expensive. An alternative to calcium fluoride glass is VPFR polycarbonate which is optically transparent, has high tensile strength and scratch resistance making it an ideal choice for HADES. However, VPFR is very expensive (3000 NZD per $\mathrm{m}^{2}$ ) and as the final form of the window is not yet known, a cheaper alternative is needed for the initial testing. The thermal camera does not need to be used for the initial testing of HADES. Therefore a thermally transparent material is not initially required.

PCS004AR polycarbonate was used to complete the initial testing. This has a similar tensile strength and scratch resistance to the VPFR polycarbonate but is significantly cheaper (600 NZD per $\mathrm{m}^{2}$ ), however, it is not transparent to the thermal spectrum. The PCS004AR polycarbonate window was machined from a $4.5 \mathrm{~mm}$ thick sheet and is attached to HADES with 12 mounting holes as shown in Figure 3.7.

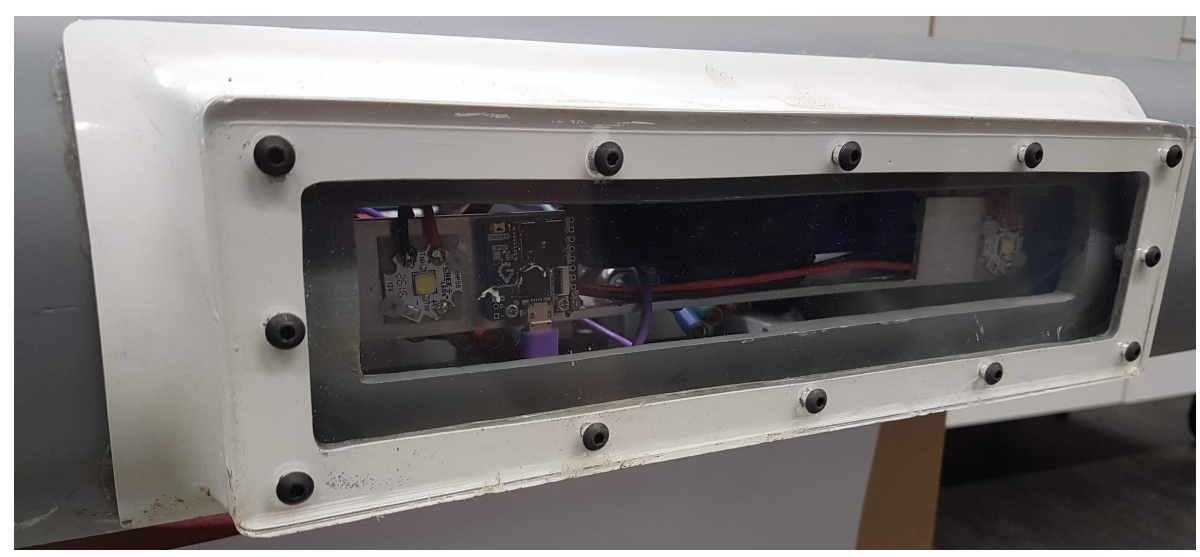

Figure 3.7: The front window and fibreglass mounting bracket.

A mounting bracket is required to mount the window material to the fibre- 
glass chassis. The bracket needs to hold the view port in front of the cameras and seal to the IP68 standard. The window material could be damaged in operation, so it needs to be easily removed and replaced.

The window mount is made from an additional piece of fibreglass that is bonded to the main chassis. The fibreglass mount has a flat section to support the windscreen material and a concave curvature to match the chassis. To allow for easy removal/replacement of the windscreen, there are $12 \mathrm{M} 4$ bolts that clamp the windscreen material to the mount. The threads for the clamping bolts are supplied by riv-nuts that are mounted to an aluminium insert bonded to the inside of the window mount. The aluminium insert also strengthens the fibreglass to prevent it distorting under the clamping pressure.

To seal the windscreen to the mount, a $1 \mathrm{~mm}$ silicone gasket is placed between the mount and the windscreen. The $12 \mathrm{M} 4$ clamping bolts provide an even clamping pressure over the surface, preventing the gasket from leaking. As in the motor mounts (Section 3.1.3), the threads are sealed by fibreglassing over the rear of the nuts.

\subsubsection{Access Panels}

There are three access panels on HADES to allow for the installation and accessing of the internal componentry. To allow for easy maintenance and installation, the internal components should mount to these access panels. As the fibreglass chassis is a good thermal insulator, the ESCs and Intel NUC need to dissipate thermal energy through the access panels. 


\subsubsection{Main Access Hatches}

The two main access panels cutouts are on the top and bottom of the main chassis. These are both $500 \times 265 \mathrm{~mm}(\mathrm{~L} \times \mathrm{W})$ and have a $45 \mathrm{~mm}$ radius on each corner. The access panel is secured to the chassis with 14 M5 bolts, where threaded inserts bonded into the fibreglass receive and seal the mounting bolts.

To seal the panels, a $6 \mathrm{~mm}$ O-ring is used. A larger O-ring is needed compared to the motor mounts as the O-ring groove is moulded into the fibreglass and has a potentially larger depth variance compared to the machined aluminium groove for the motor mounts (Section 3.1.3). The O-ring for the main panels is also larger as the required feature size for a $3 \mathrm{~mm}$ O-ring groove is below the resolution achievable for the fibreglass moulding process used (Section 3.1.7).

After the bolt hole pattern and O-ring groove have been added, the remaining workable area of the main panels is reduced to $440 \times 205 \mathrm{~mm}$. To allow for heat to dissipate from the ESCs and Intel NUC, the access panels are made from machined aluminium. However, to reduce the cost, for initial testing the main access panels are constructed from laser cut acrylic. Once the position and function of each internal component has been tested and confirmed, the final aluminium panels can be machined.

The top access panel will be the most commonly opened panel on HADES as it is where the batteries are installed and accessed through for recharging. Attached to the top access panel is the Intel NUC, the positive pressure gas system, the main control PCB and the sensor pod. To allow for easy mounting, the NUC, main control PCB and gas system are mounted to a gear-tray which allows for quick replacement of these systems. It also allows the component placement to be redesigned without having to machine a new aluminium cover plate. 


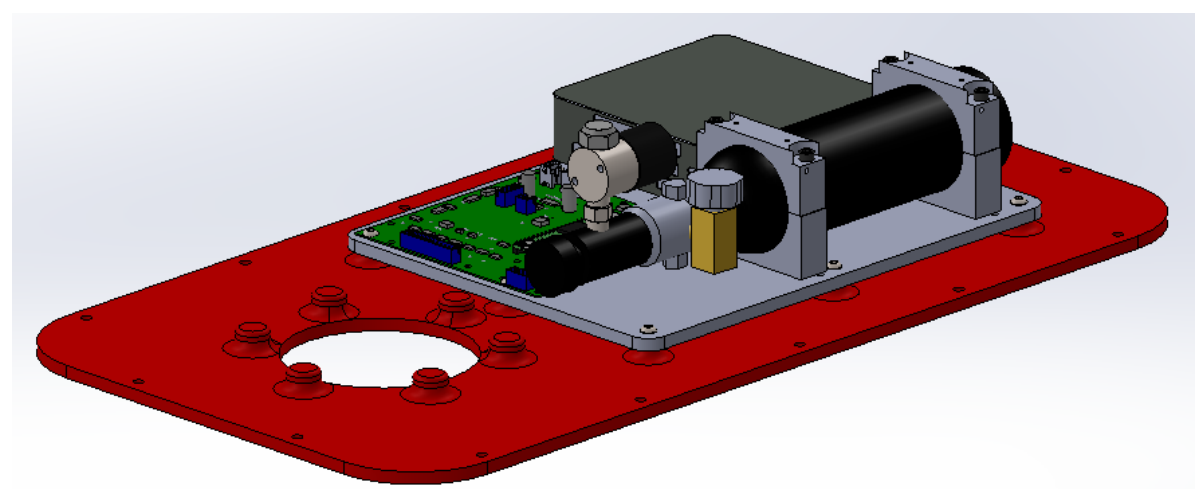

Figure 3.8: The CAD model of the top access panel assembly without the sensor pod installed.

The gear-tray is mounted to the panel with nine M4 mounting bolts that thread into blind holes in the $6 \mathrm{~mm}$ aluminium panel. The electronics and gas system mount to the access panel with M3 and M4 bolts though the back of the gear-tray into the components. The NUC and the gas system are both supported with 3D printed brackets to secure them during the high amplitude vibrations caused by the Whegs.

The sensor pod (Section 3.4) mounts to the forward section of the top panel. It is secured with the same style of mounting hole pattern as the motor mounts. This allows the same O-ring to be used. Figure 3.8 shows the CAD design of the top panel.

The bottom access panel provides mounting for the ESCs, the control batteries, the power management PCB and the rear arm actuation system. The ESCs, battery mount and power PCB are mounted to the access panel with M3 bolts threaded into blind tapped holes. To allow for good heat transfer between the ESC heat-sink and the panel, thermal compound is spread underneath the ESCs before they are clamped down. To prevent the aluminium from contacting the bottom of the power management PCB and causing a short circuit, a laser cut piece of plastic is placed underneath the $\mathrm{PCB}$ isolating it from the aluminium. Figure 3.9 shows the CAD model of the bottom panel. 


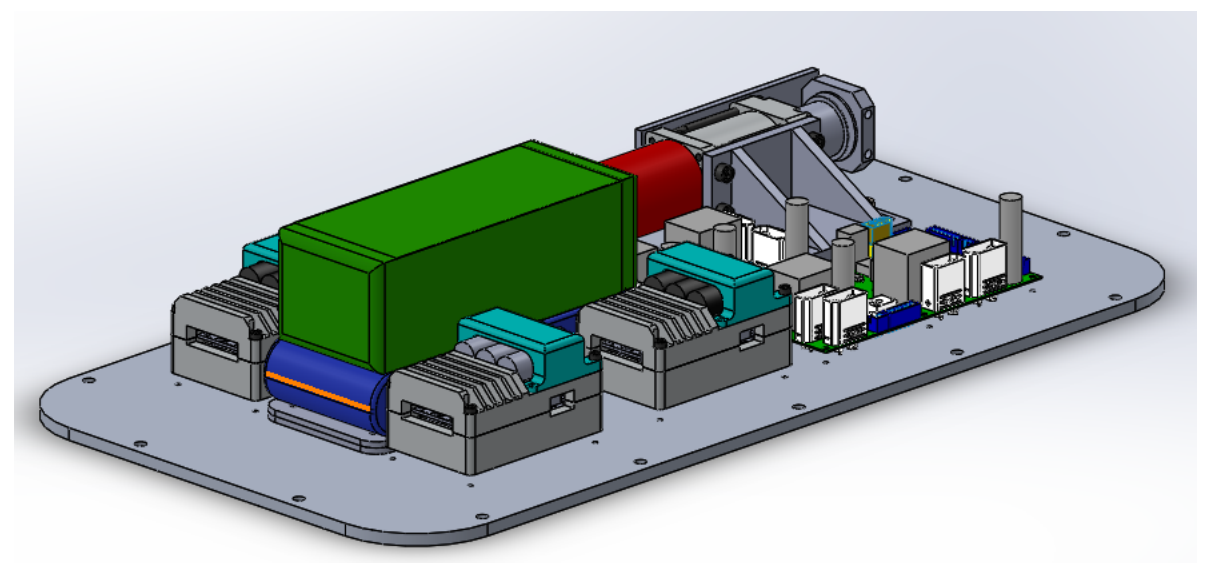

Figure 3.9: The CAD model of the bottom access panel the with the ESCs, power management $\mathrm{PCB}$ and the rear arm actuation system.

The rear arm articulation system will be under high load when it is active. To prevent the M5 bolts from pulling out from the aluminium panel, or the panel distorting, a second $6 \mathrm{~mm}$ plate is welded onto the access panel. The second plate increases the thread depth of the bolts and increases the rigidity of the access panel surrounding the actuation system.

\subsubsection{Rear Access Panel}

The rear access panel mounts at the back of the robot on the rear arm. Its primarily function is to allow access for running cables though the rear arm pivot assembly (Section 3.2). It also serves as a mounting point for the rear looking camera, positive pressure fill and purge valves and the debug connector.

The rear access panel is made from machined $10 \mathrm{~mm}$ aluminium, it mounts to the chassis with the same system as is used for the motor and rear arm pivot mounts (Section 3.1.3). The panel is sealed to the chassis with a $3 \mathrm{~mm}$ O-ring that is compressed between the chassis and panel. The six M5 mounting bolts are counter bored into the surface to prevent them from interfering with the gas 
connections

There are two connections on the rear panel for the positive pressure gas system; one for filling the chassis and one for purging the chassis. As discussed in Section 3.3, the two gas connections should accept a 1/8 NPT gas fitting. It is likely that the fitting used will be steel. To prevent these fittings from damaging the aluminium panel, steel threaded inserts are used. These are threaded into the aluminium panel and are locked in place with lock nuts. On the inside of the steel threaded inserts, the two control solenoids are mounted which are held in place with a rigid $1 / 8$ NPT joiner.

To allow the camera to see outside the chassis, a $4.5 \mathrm{~mm}$ polycarbonate window is inset into the aluminium housing. The window is secured with ten M3 bolts that are threaded into blind tapped holes in the aluminium. The window is sealed with a $1 \mathrm{~mm}$ silicone gasket that is compressed between the windscreen material and the aluminium housing with the mounting screws. The camera is mounted behind the window with a 3D printed ABS bracket that also secures the illumination LEDs.

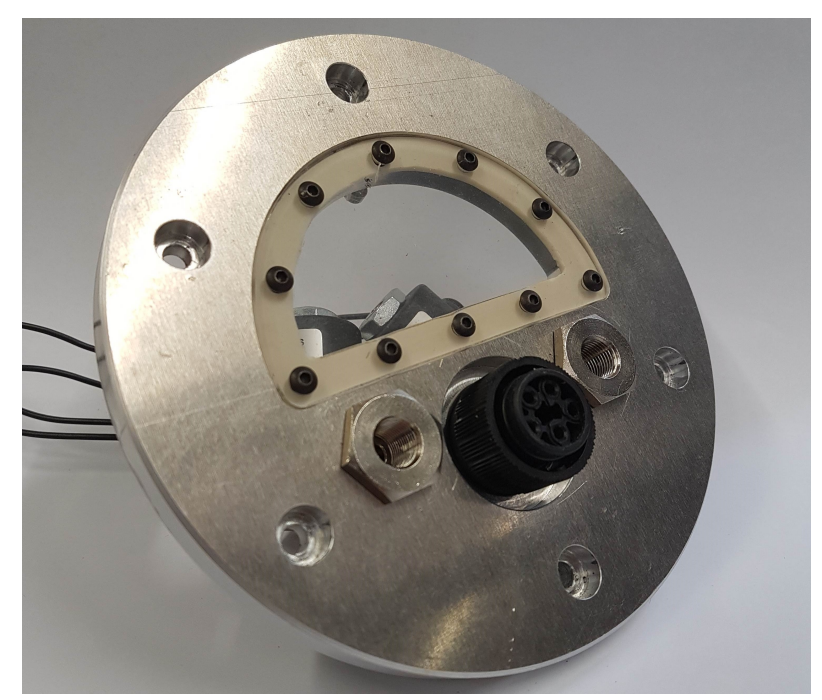

Figure 3.10: The rear access Panel without the camera or illumination system installed. 
The debug connector allows connection to the internal chassis electronics without opening the chassis, should the communication systems fail. As the connector could be in contact with a potentially explosive atmosphere, it must meet the A/NZ60079.11 safety standard [11]. This standard states that the connector contacts need to have a spark distance of greater than $3 \mathrm{~mm}$. To meet this requirement, the RST mini 5 Wieland connector was chosen. The RST mini- 5 is a locking connector that also meets the IP68 standard and has five contacts.

To protect the connector from physical damage, it is recessed into the panel. The mounting hole is keyed so that the connector will not rotate when inserting the mating cable connector. The connector is secured into the chassis with a locking nut and sealed with an integral gasket. Figure 3.10 shows the rear access panel.

\subsubsection{Battery Mounting}

The lithium polymer (Li-Po) batteries that are used in HADES are potentially flammable if they are ruptured or placed under excess physical stress. The battery mounting system must therefore protect the batteries and prevent them from moving when HADES is in operation. The battery mounting system must also allow the batteries to be removed for charging or replacement.

As the batteries cannot be ruptured, screw fixings cannot be used to secure them. Instead the batteries are held down with Velcro tape. Four of the batteries are held in the spaces beside the main hatches. To support these batteries, a laser cut piece of acrylic is bonded onto the chassis on 3D printed ABS brackets. The batteries are then strapped to this acrylic plate with Velcro. To prevent the batteries from sliding out of the straps, 3D printed brackets are mounted at the end of the acrylic plates. Figure 3.11 shows the battery mounting.

The remaining two batteries are stacked and then strapped to an acrylic 


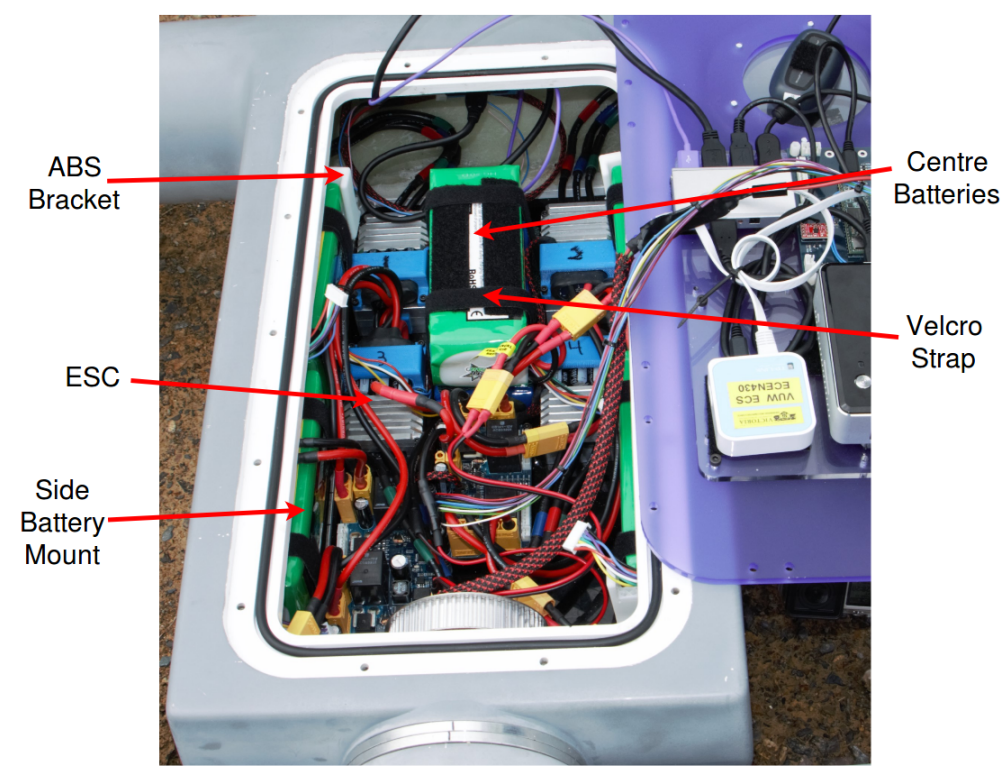

Figure 3.11: The batteries mounted inside HADES during the field trails.

bracket on the bottom panel. As there is no space around these batteries to stop them from sliding out of the straps with a physical stop, non slip matting is used. The matting is placed between each battery and between the mounting plate and the bottom battery. When the compression from the straps is applied the batteries cannot move. Additionally, adhesive-backed Velcro tape is applied to the batteries which bonds with the Velcro hold-down straps.

\subsubsection{Design for Manufacture}

The HADES fibreglass chassis is formed using a moulding process that was contracted to Solpont Composite Manufacturing. Constructing the moulds for the chassis is done in two steps; first a mould plug is machined, then a mould is cast from the mould plug. One mould plug is typically used to make many moulds. To manufacture the fibreglass components, Solpont requires 3D models of the mould plugs. The chassis is constructed from four bespoke mould plugs; the main chassis, the rear arm, the view port mount and the motor end 
caps. The mould plugs are machined from tooling board; a high density easily machinable foam.

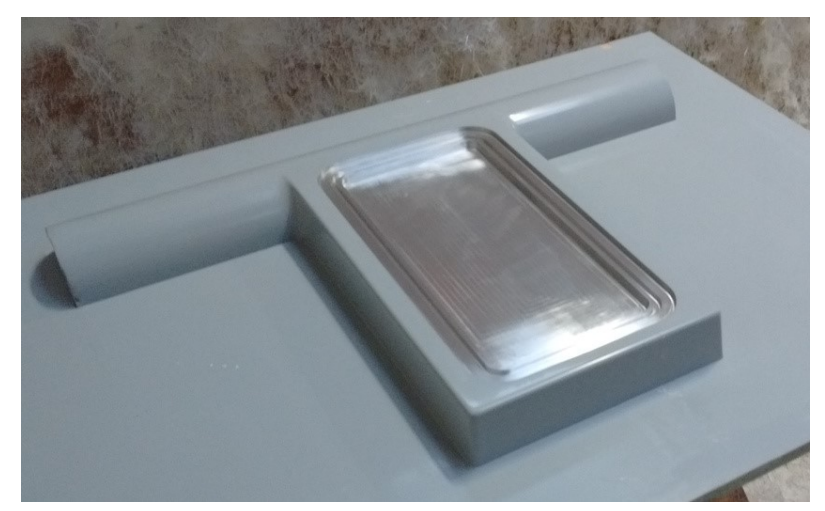

Figure 3.12: The mould plug with the aluminium insert for the main chassis fibreglass component.

A mould plug consists of the main chassis shape against a flat plane. When the mould is formed from the mould plug the result is a flat surface with an indent that matches the required component. The fibreglass and epoxy resin are then laid in the indent and cured. When the fibreglass is removed from the mould it matches the form of the mould plug.

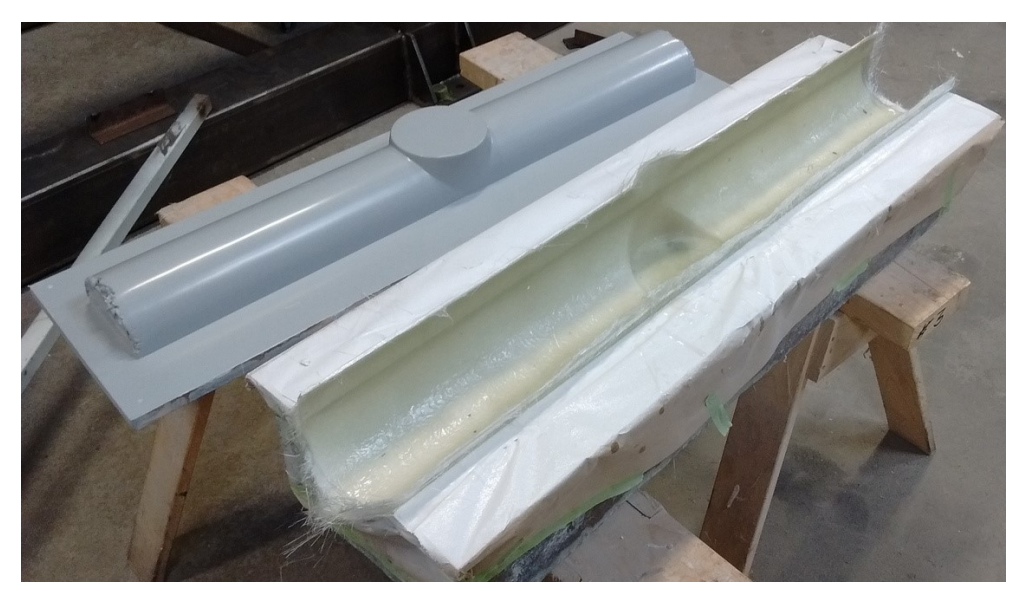

Figure 3.13: The mould plug and mould with a fibreglass part curing in the mould, the indexing tab can be seen on the far side of the moulded part. 
The main chassis mould plug is the largest moulded component, it also has the smallest feature size for the O-ring used to seal the main hatch. Small features made from tooling board can be easily broken when the plug is removed from the mould. To prevent breakages from occurring, the main hatch features are machined from an aluminium insert that is then added to the tooling board mould plug. To maintain the $3^{\circ} \mathrm{draft}$ angle, the aluminium insert was machined with a customised tapered end-mill, reducing the machining time and cost. Figure 3.12 shows the mould plug for the main chassis with the aluminium insert.

The rear arm mould is similar to the main chassis mould, except the symmetry plane for the moulded components is rotated to better suit the parts' geometry. To index the clam-shell parts together, a tab is run around half the outside of the mould. This tab then interlocks with the other mating component. As well as aligning the parts together, the tab helps with forming a seal and increasing the contact area of the gluing surface. The mounting tabs can been seen in Figures 3.13 and 3.14.

The majority of the features on HADES are designed with a draft angle, however, the motor mounts need to be square to the chassis and hence a separate component is required. Constructing the motor mounts as separate components allows easy mounting of the steel reinforcing rings in the chassis. The part has cutouts for the tabs that keep the main chassis halves indexed together.

The mould for the motor mounts is constructed from two interlocking pieces that form a cavity matching the shape of the motor end cap. The mould splits along the axis of the tube allowing the finished piece to be removed. To precisely drill the mounting holes in the fibreglass motor end caps, a drill guide was cut from steel. This ensures that the holes are precisely centred and repeatable over all the end caps. Figure 3.14 shows an exploded view of the motor end caps and chassis halves. 


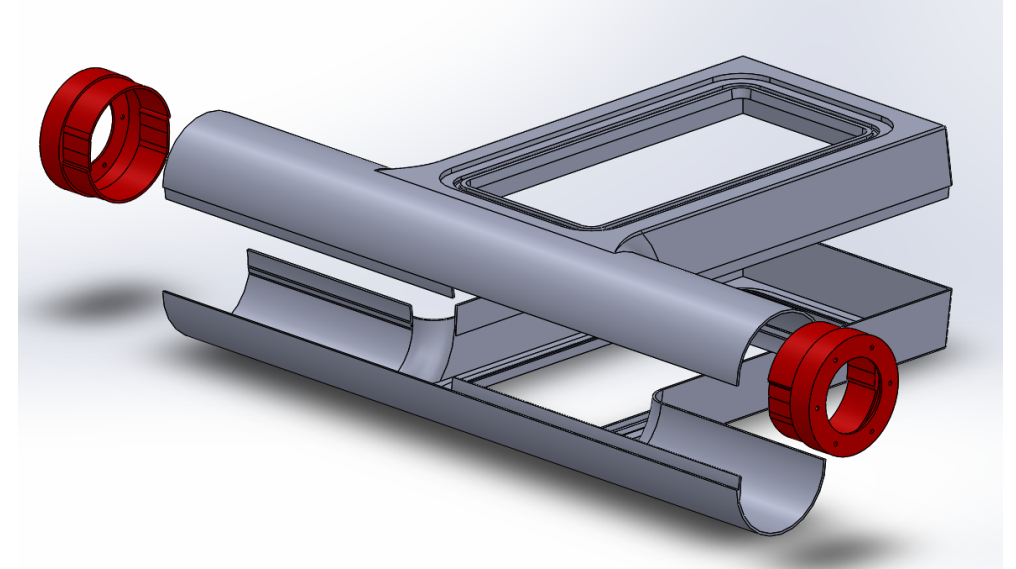

Figure 3.14: An exploded view of the chassis components showing the alignment tabs and motor end caps.

\subsection{Rear Arm Pivot}

Allowing the rear arm of the chassis to pivot increases the rough terrain ability of HADES as the rear Whegs can adapt to the terrain. Actuating the rear arm pivot was identified as a way to increase the usefulness of the pivot, as the Whegs could be lifted onto obstacles or the robot self-righted [49].

The rear arm pivot needs to allow the high current motor cables and the data cables to pass between the chassis and the rear arm. Due to the high number of connections and the high motor currents, a slip ring cannot be used. Cumulatively all the cables passing through to the rear arm are approximately $25 \mathrm{~mm}$ in diameter. The arm actuation system (Section 3.2.1) will need to connect to the rear arm though the pivot. To facilitate this a 60 tooth, $15 \mathrm{~mm}$ T5 belt pulley needs to be mounted to the pivot component that is connected to the rear arm.

The pivot mechanically links the drive forces from the rear arm with the drive forces from the main chassis and therefore will be subjected to high radial and axial loads during operation. The pivot will need to withstand these loads 
with minimum deformation.

The rear arm pivot follows the design from the pivot developed for revision one of HADES [49]. The original HADES pivot was constructed from two aluminium tubes that rotated concentrically inside one another. The two tubes were retained axially with a shoulder bolt. The shoulder bolt also served to limit rotational travel to $120^{\circ}$. Figure 3.15 shows the rear arm pivot developed for HADES revision one [49].

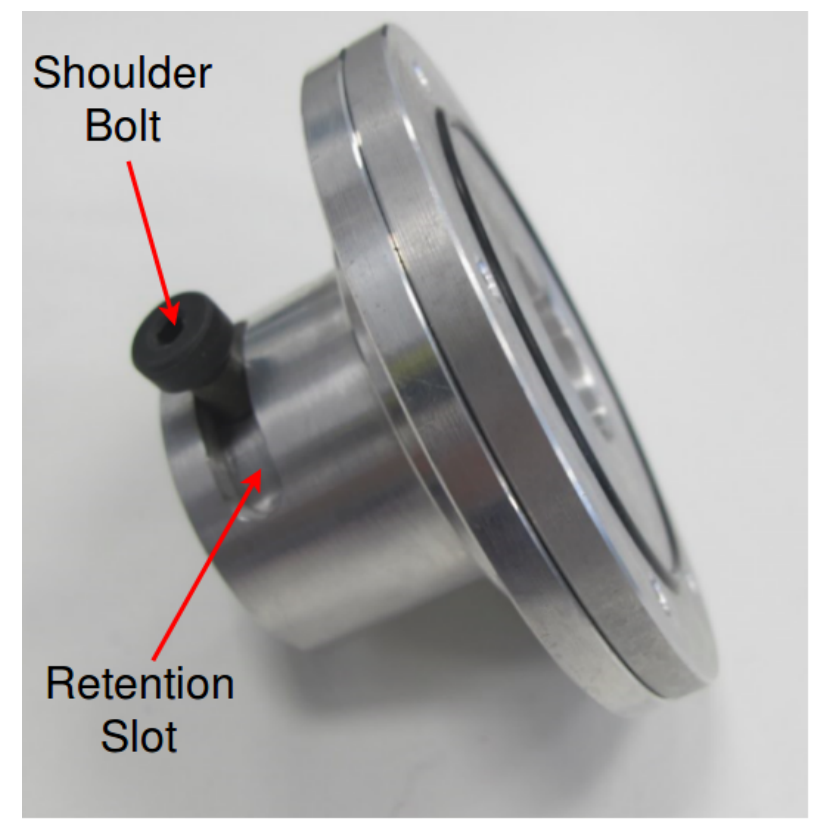

Figure 3.15: The pivot assembly used in HADES revision one [49].

During the trials of HADES revision one, the axial forces on the pivot caused the shoulder bolt to damage the aluminium slot it travels in. It was also noted that the $120^{\circ}$ travel was restrictive when travelling over large obstacles. The revision one pivot is too small for the new chassis design (Chapter 3) and a larger more robust design is needed.

The new pivot system has an internal diameter of $60 \mathrm{~mm}$ and the pivot tube is $74 \mathrm{~mm}$ long, with a nominal wall thickness of $6 \mathrm{~mm}$. The larger dimensions 
(compared to the revision one design), increase the strength and distribute the loads over a much larger surface area. To mount the pivot assembly to the chassis and rear arm, 12 M5 countersunk bolts are used and these attach to the mounting system covered in Section 3.1.3. To meet the IP68 standard, the pivot is sealed with a $3 \mathrm{~mm}$ diameter O-ring. The rotating pivot assembly is sealed with a low pressure lip seal, which is pressed into the outer tube of the pivot assembly.

To spread the axial load over a larger area than the revision one pivot, the inner tube of the pivot is axially retained in the outer tube by the belt pulley. The pulley is bolted onto the front on the inner tube with eight M4 bolts. To account for the M4 bolts, the wall thickness of the inner tube is $10 \mathrm{~mm}$. The inner tube has a $40 \mathrm{~mm}$ diameter bore to allow the cables to pass though freely. Figure 3.16 shows a cross section view of the pivot assembly.

Instead of mechanically limiting the maximum angle of the pivot, the actuation system will monitor and engage when the angle reaches a user defined threshold. This allows the user to limit the maximum angle depending on the encountered terrain.

When two aluminium surfaces are moving and are in contact under high pressures, they can gall. Galling is when the running surfaces are damaged, as the pressure causes them to fiction weld and then to separate, leaving pitting on the surfaces [27]. The main barrel of the pivot assembly has a relatively large surface area and therefore the surface pressures will be low. However, the axial retaining force for the pivot assembly is applied between the actuation pulley and the end part of the pivot. This is a small surface area and therefore galling is likely.

To mitigate the galling effect between the pulley and the pivot assembly, a custom laser cut steel flange was added to the pulley. A steel and aluminium running surface is less likely to gall as steel is much harder than aluminium [27]. The steel flange also keeps the drive belt from falling off the pulley. Due to the 


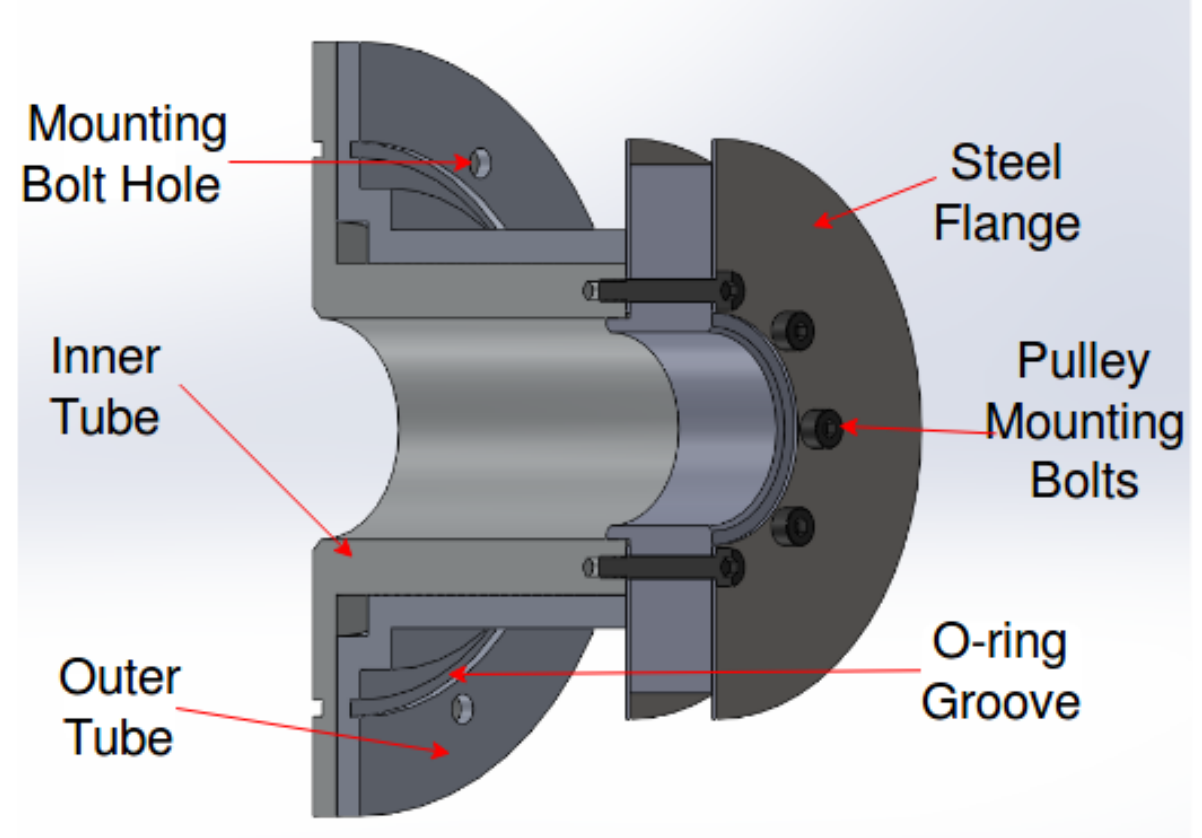

Figure 3.16: A labelled cross section of the aluminium rear arm pivot.

hardness difference, it is likely that the aluminium pivot assembly will wear faster than the pulley flange, reducing the total operational life of the pivot assembly. To additionally prevent galling between all the aluminium surfaces lubrication is needed. To lubricate the running surfaces Molyslip grease is used. To allow grease to be added to the pivot without disassembling it, a grease nipple is added to the outer tube.

The aluminium pivot design as the potential to gall during operation, a much better design would be bronze and steel design. However, as HADES is a prototype and a bronze and steel design is significantly more expensive the aluminium pivot is manufactured. The outcomes from testing the pivot system can be seen in Section 9.3.1. The finished pivot can be seen disassembled in Figure 3.17. 


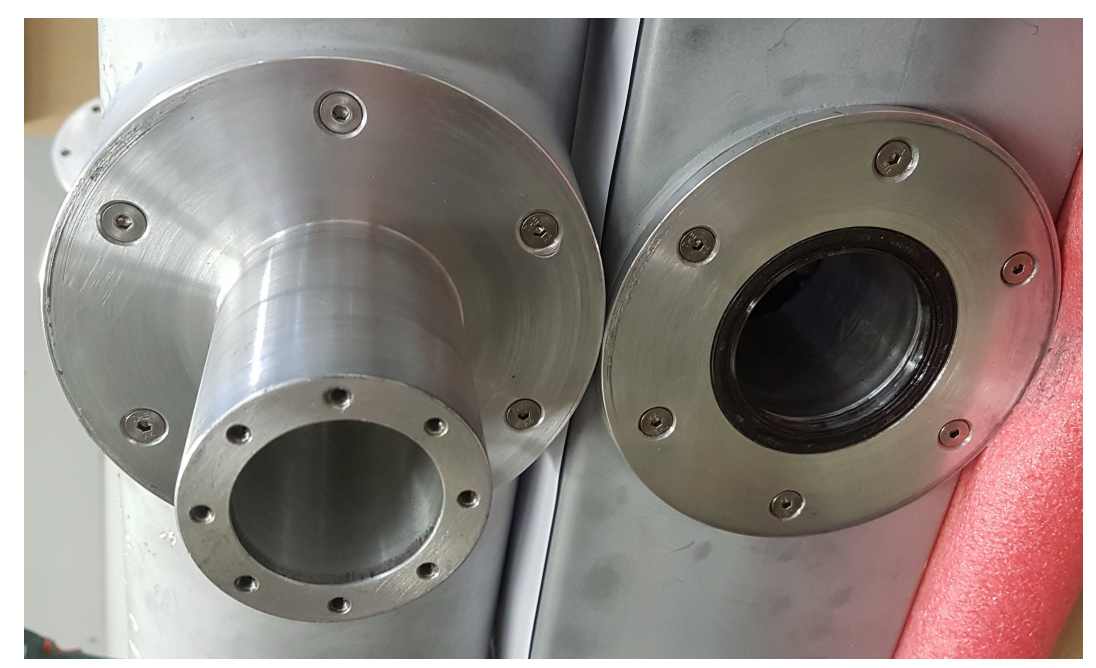

Figure 3.17: The dissembled rear arm pivot used in HADES.

\subsubsection{Rear Arm Actuation}

To actuate the rear arm, the pivot assembly needs to be connected to a drive system. The drive system will need to be compact and be able to provide the necessary torque to move the weight of HADES. As small electric motors cannot supply large amounts of torque, a reduction gearbox is needed. The maximum expected torque at the pivot is given by Eq. 3.2.

$$
\tau_{p}=1.25 \mathrm{mg} r_{a r m}
$$

where $\tau_{p}$ is the torque at the pivot, $m$ is the mass of the chassis, $g$ is the acceleration due to gravity and $r_{\text {arm }}$ is radius of the rear arm. A factor of 1.25 is included in the equation to account for the frictional losses in the rear arm pivot. The length of the rear arm is $800 \mathrm{~mm}$, so the radius of the pivot is $400 \mathrm{~mm}$ and HADES weighs $30 \mathrm{~kg}$, therefore, the torque at the pivot is $147 \mathrm{Nm}$.

To connect the rear arm pivot to the actuation system, a $15 \mathrm{~mm}$ wide T5 toothed belt is used. To reduce the torque from the pivot before the gearbox, a 
60 tooth pulley is used on the pivot, driven by a 20 tooth pulley on the actuation system. The two pulleys provide an initial torque reduction of 1:3, therefore the actuation system only needs to provide $49 \mathrm{Nm}$ of torque.

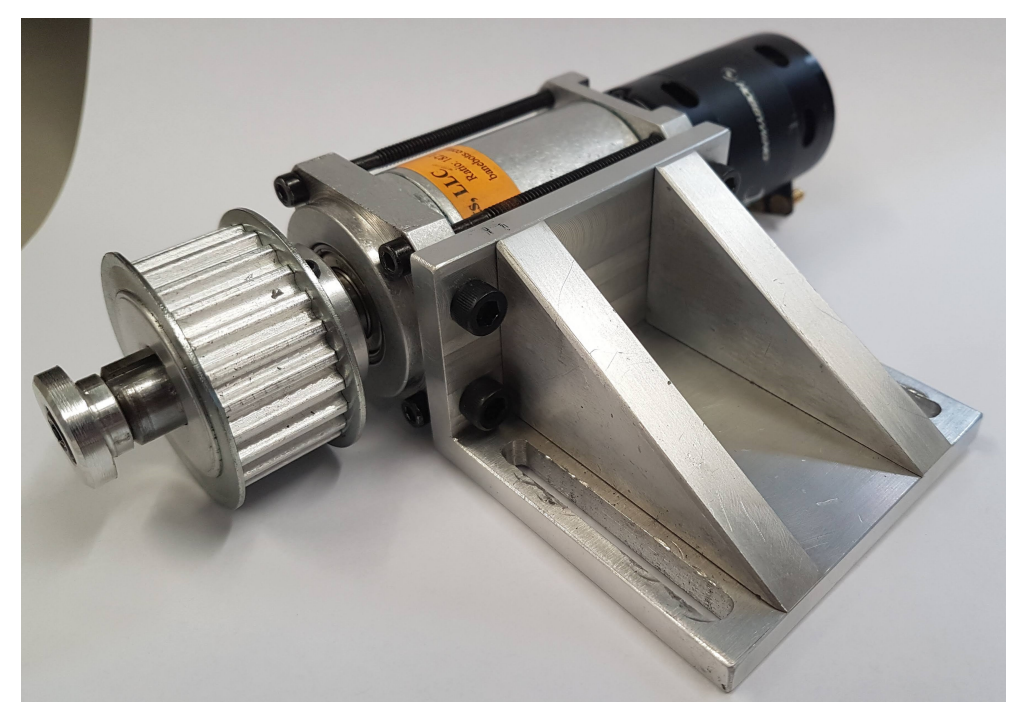

Figure 3.18: The drive unit for the rear arm articulation with the BaneBots P60 182:1 gear box and Justock brushless motor.

To supply the torque to the 20 tooth pulley, a BaneBots 182:1 P60 gearbox is used. The P60 series of gearboxes have a maximum output torque of $61 \mathrm{Nm}$. To drive the BaneBots gearbox, a Justock 3650 brushless motor is used. The Justock motor also has an encoder to provide feedback for the pivot's position. After the reduction from the pulley system and the gearbox, the peak load at the motor is $0.27 \mathrm{Nm}$ which can easily be driven by the Justock motor. The motor, gearbox and drive pulley are illustrated in Figure 3.18.

The drive system will be under high loads when it is active and therefore a secure mounting is needed to mount it into the chassis. To attach the drive system to the chassis, a custom angle plate was machined. The angle plate secures to the BaneBots gearbox with four 10-32 bolts and to the chassis with four M5 bolts. The M5 bolts mount in slots to allow the plate to slide which results in the tension of the belt to be adjusted. 


\subsection{Positive Pressure Gas System}

To meet the A/NZ60079.11 standard the chassis needs to be kept at a higher pressure than the surrounding atmosphere by $50 \mathrm{~Pa}$. The atmosphere inside the chassis must also be inert and contain less than $1 \%$ oxygen. The inert, higher pressure atmosphere must be maintained while HADES is inside the underground mine. A system is needed on HADES to monitor and control the pressure/atmosphere inside the chassis.

The gas system needs to allow the chassis to be filled with an inert gas. It also must allow a small volume of gas to be carried on HADES in case the chassis develops a leak and the pressure needs replenishing. The gas pressure in the chassis needs to be carefully controlled with the control electronics. An over-pressure event could damage the chassis and internal components.

The atmosphere in the chassis must be inert and contain less than $1 \%$ oxygen. To achieve this, the existing atmosphere in the chassis needs to be purged and replaced with an inert gas. Three commonly available inert gases are carbon dioxide, argon and nitrogen. The gas sensor system samples the carbon dioxide concentrations outside the chassis. Therefore, carbon dioxide cannot be used inside the chassis as it would contaminate the sensor reading.

Nitrogen is significantly cheaper than argon gas as it is a lot more common in the atmosphere. Nitrogen gas is also used in underground mines already to purge explosive gases out. Therefore, nitrogen is used as the inert gas to fill HADES. Nitrogen gas can be purchased in two forms, wet and dry. Wet nitrogen contains water molecules in solution with the gas. As the water could condense out and potentially damage the internal electronics, dry nitrogen is used.

Before deployment the chassis will need to be filled with nitrogen, filling the chassis will also purge any non-inert gases out. To allow for the filling and 
purging of the chassis two solenoid valves are used, these are both mounted to the rear access panel (Section 3.1.5.2). Internally a pipe is used to take the incoming nitrogen to the front of the chassis, where it will push the remaining gases in the chassis towards to the purge valve at the rear. To prevent a high-velocity stream of gas from damaging internal components or objects surrounding the chassis, sintered brass gas diffusers are fitted to both the fill and purge gas lines.

Ideally, the chassis will not leak, and the pressure will be maintained throughout the mission. However, as the pressure differential is critical for HADES to operate inside an underground mine a backup system is needed. The system needs to be able to replenish the gas in the chassis should the pressure drop or the oxygen concentration increase.

To carry the gas inside the chassis, a 13 cubic inch $\left(213 \mathrm{~cm}^{3}\right)$ paintball tank is used as a reservoir. The paintball tank can be pressurised to up to 3000 psi (20.7 MPa). If the chassis is pressurised at $100 \mathrm{~Pa}$ above atmospheric pressure (twice the standards requirements), the tank can carry enough gas to fill the internal volume $\left(0.048 \mathrm{~m}^{3}\right)$ of HADES 1.5 times. To control the gas flow into the chassis and prevent over-pressurisation, a B071ZNP5BS regulator is used, which reduces the pressure to 30 PSI. A solenoid valve is then used to control the gas flow into the chassis.

To monitor the pressure in the tank and obtain an approximate fill level, a TE Connectivity M5231 sensor is used. To connect all the system components together, a custom manifold was machined. This can be seen with the rest of the internal gas system in Figure 3.19. 


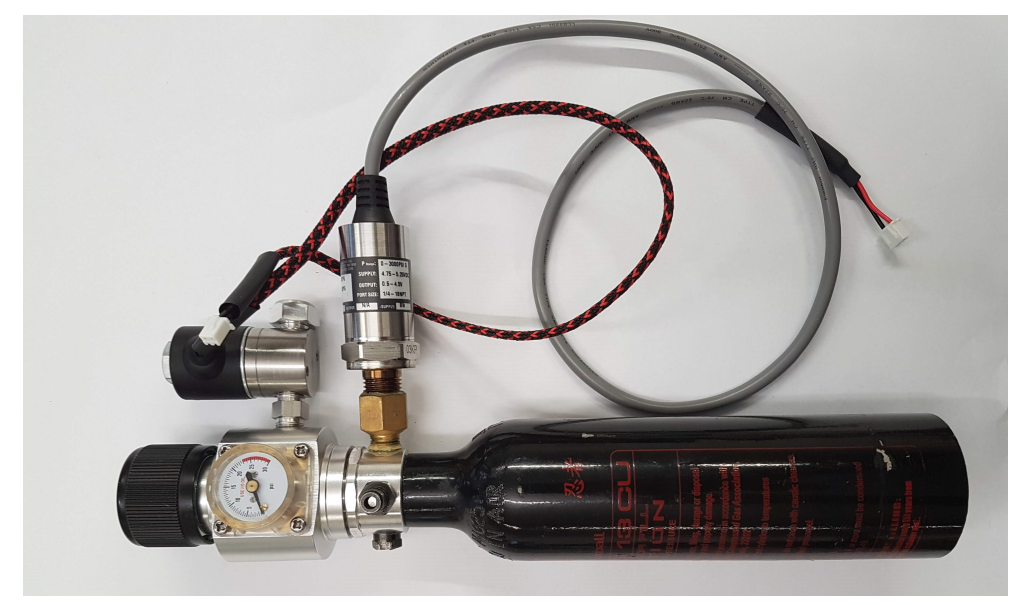

Figure 3.19: The internal tank and regulation system for the positive pressure gas system.

\subsection{Sensor Pod}

The hazardous gas and other atmospheric sensors (Chapter 5) need a mounting point on HADES that is protected from physical damage but still has high air flow. The sensor mount will need to support the sensors and their electronics and isolate them from the vibration from the Whegs. The sensor mounting system will need to seal the sensors and chassis mount to the IP68 standard.

To mount the sensors a custom sensor pod was developed, this attaches to the front of the top access panel. The mounting bolt hole and sealing configuration is similar to the one used for the motor mounts (Section 3.1.3) which allows the same O-ring and fasteners to be used. The sensor PCB is mounted inside the sensor pod with five standoffs, the sensors then mount to this PCB and protrude through the top of the sensor pod. To prevent water from flowing through the sensor cut-outs, they are sealed with a $1.5 \mathrm{~mm}$ O-ring. Additionally, to prevent water pooling next to the sensors, the top of the sensor pod is slightly domed so the water will flow off. Figure 3.20 shows an internal view of the sensor PCB mounting to the back of the top plate. 


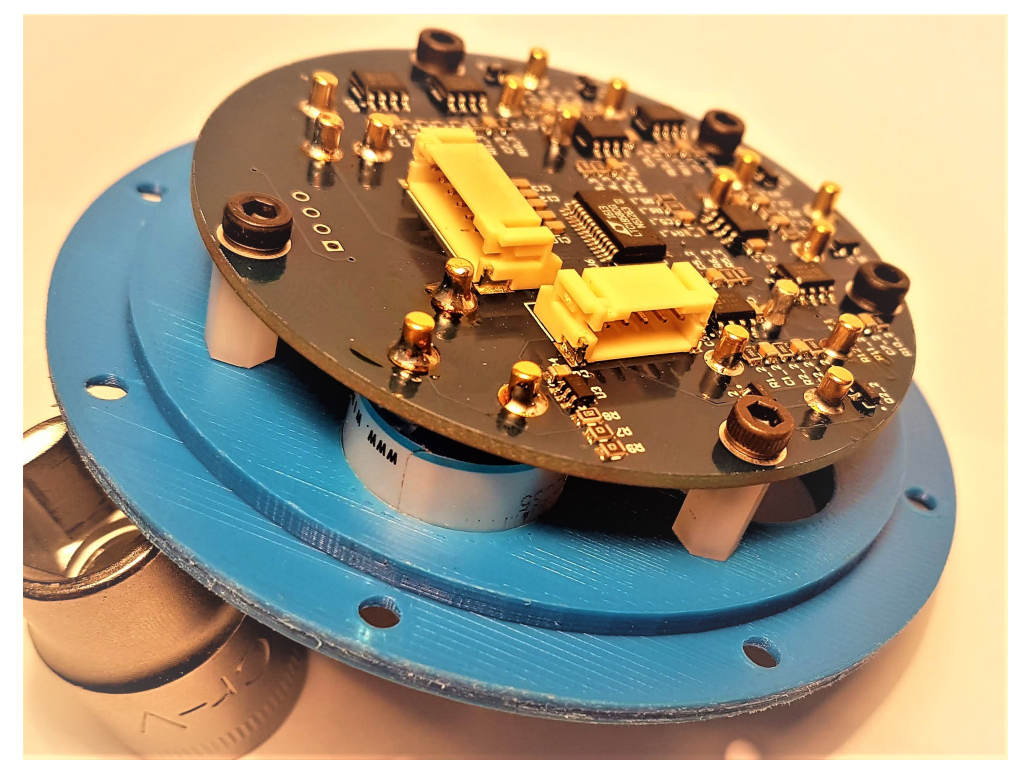

Figure 3.20: The sensor PCB and sensors mounted to the top plate of the sensor pod.

To protect the sensors from physical damage the sensor pod is protected by a steel flange. This protects the sensor from falling debris but is open on the sides to allow air flow. A 3D printed prototype of the sensor pod can be seen in Figure 3.21.

\subsection{Summary}

The chassis design of HADES enables it to successfully and safely enter and navigate through an underground mine after a disaster event. The chassis is manufactured from a fibreglass material that is both strong and lightweight. The HADES chassis houses all the electronics and drive systems internally. Due to a number of components being housed internally, there are multiple access panels installed in HADES chassis. The access panels allow internal components to be mounted, provide thermal dissipation of the internal com- 


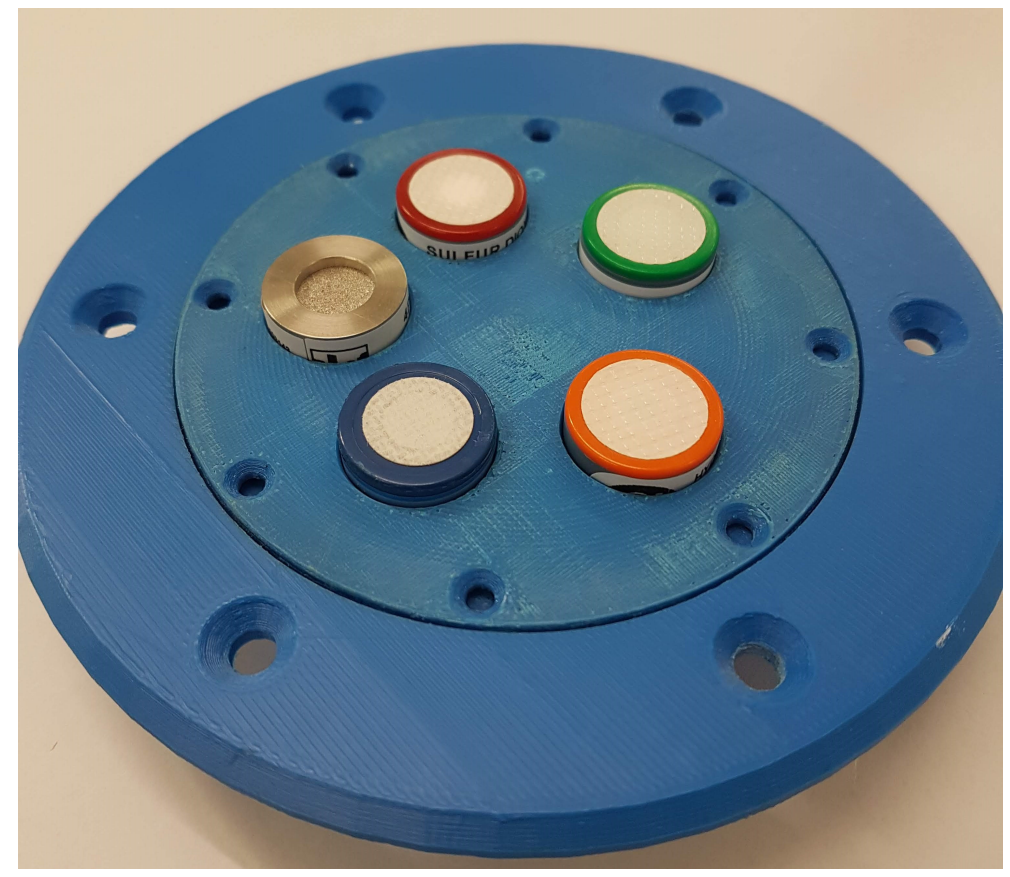

Figure 3.21: A 3D printed prototype of the sensor pod without the steel over the sensor protruding out of the top plate.

ponents, and allow maintenance access. To improve the rough terrain capability of HADES, the rear arm can pivot. This pivot is actuated to increase the capabilities of HADES further. 


\section{Chapter 4}

\section{Mechanical - Locomotion}

\subsection{Wheel Design}

Chapter 2 identified that wheeled locomotion systems perform poorly over rough terrain, and tracked systems are complex and therefore expensive. Spokedwheels or wheel-legs (Whegs) were identified as a suitable replacement. Whegs (like wheels) only require one rotating member to generate locomotion and are therefore simple and low cost to implement. Whegs also have a much better rough terrain climbing capability compared to wheels, as the spokes can "grab" onto obstacles to climb over them. Depending on the shape of the spokes, Whegs can also be used for paddling through water.

\subsubsection{Specifications}

Three aspects of Wheg design determine their rough terrain climbing capability: duty cycle, diameter and material selection. The chassis must have at least $150 \mathrm{~mm}$ of ground clearance in order to climb stairs and the train tracks 
commonly found in underground mines (Section 2.1). The HADES chassis is $140 \mathrm{~mm}$ in depth (Chapter 3), and so to meet the ground clearance requirement, the Whegs must not be smaller than $440 \mathrm{~mm}$ in diameter. The largest Wheg diameter is determined by the axle spacing between the forward and rear axles $(640 \mathrm{~mm})$ and so is limited to a maximum diameter of $590 \mathrm{~mm}$, allowing $50 \mathrm{~mm}$ clearance between the forward and rear Whegs.
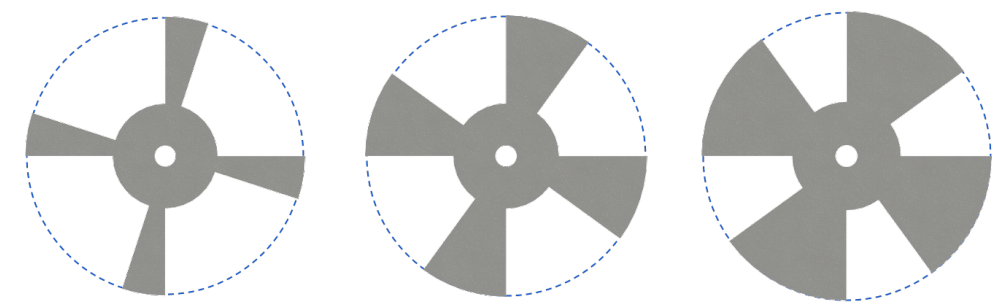

Figure 4.1: A four spoke Wheg with $20 \%, 40 \%$ and $60 \%$ duty cycle (left to right).

The duty cycle is the percentage of the circumference that is supported by the spoke of a Wheg. Figure 4.1 shows three different duty cycles for a fourspoke Wheg. The duty cycle provides a trade-off between the size of obstacles that can be climbed and the flat ground speed/ride smoothness. To determine a suitable compromise between rough terrain capability and ride smoothness, initial testing was undertaken.

The initial testing was carried out with the Whegs developed for HADES revision one [48]. These are made from $18 \mathrm{~mm}$ BS1154 rubber strengthened with two $6 \mathrm{~mm}$ aluminium plates and have a diameter of $400 \mathrm{~mm}$. The Whegs used were in a five-spoke configuration, with hammerhead rubber tips on each spoke. Figure 4.2 shows a CAD model of the Whegs used in the initial trials.

The trials were carried out on varying ground topography to ascertain the Whegs' capability to navigate rough terrain. The 33\% duty cycle of the Whegs performed well over all the tested terrains and inclines. The Whegs were able to gain traction on all surfaces and vertical vibrations were minimal. However, 


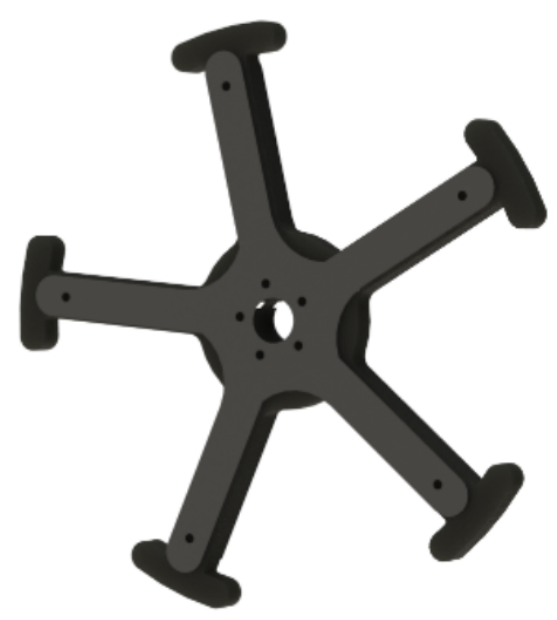

Figure 4.2: The Whegs used for the initial trials.

if all four Whegs became in phase with one another, HADES would gain a large amount of vertical movement on the downward stroke of each spoke.

The hammerhead tips performed poorly as they deformed under load. The deformation caused the tips to become caught on rocks when traversing rough terrain and when performing skid turns. As the loads on the tips changed, the rubber in the spokes also deformed. This caused the spokes to twist and forced the aluminium plates and rubber to delaminate. The testing indicated that a new design of the Whegs could significantly improve their capability.

\subsubsection{Implementation}

As discussed in [48], it is not possible to develop a Wheg that can navigate over all terrain types. Rather the Wheg design should be selected for the type of terrain that HADES will be traversing. As the terrain may differ between mines, it may be required to swap out the installed Whegs to Whegs that are more suited to the terrain HADES will be traversing. To allow for quick Wheg changes, a quick mount system was developed which is discussed in Section 4.2. The 
Wheg attaches to the mounting system with eight M8 bolts.

As mentioned, the initial trials identified that when the Whegs become in phase, HADES would gain large vertical oscillations on the downward stroke of each Wheg. To mitigate this, the rear Whegs were moved to a six spoke system. Thus, the five-spoke front Whegs could never stay in phase with the rear Whegs when they are rotating at a similar speed. Increasing the number of spokes increases the duty cycle from $33 \%$ to $40 \%$, decreasing rough terrain capability of the rear Whegs. However, this duty cycle reduction is not an issue as most of the large obstacle climbing will be done with the front Whegs and HADES can lift itself with one Wheg (Section 4.2).

The $400 \mathrm{~mm}$ diameter Whegs used in testing do not meet the ground clearance requirements, therefore, a larger Wheg is needed. To allow for a ground clearance of $215 \mathrm{~mm}$ ( $65 \mathrm{~mm}$ more than the minimum specification), a $570 \mathrm{~mm}$ diameter Wheg was designed. To support the rubber in the Wheg, $6 \mathrm{~mm}$ aluminium supports are used, as these performed adequately in the initial testing.

The initial trails also identified that the strength of the rubber needs to be increased to prevent deformation. To replace the BS1154 rubber, a $25 \mathrm{~mm}$ fibre reinforced FRNR rubber was selected. The FRNR rubber has a similar coefficient of friction to the BS1154 rubber, so it will still maintain traction on smooth surfaces. The FRNR rubber also includes reinforcing, strengthening the rubber and limiting deformation. To stop the rubber from deforming along the spokes of the Whegs, two additional $8 \mathrm{~mm}$ bolts were added along the spoke to clamp the $25 \mathrm{~mm}$ rubber between the aluminium supports.

The hammerhead tips on each of the spokes performed poorly in the initial trials. These tips were originally added to dampen the impact when the spoke came into contact with the ground on its downward stroke [48]. To achieve the same dampening effect, the rubber tips were rounded with a $30 \mathrm{~mm}$ radius. To allow for the rubber to dampen and grip the ground, it protrudes $10 \mathrm{~mm}$ from the aluminium support around the tip. Figure 4.3 shows an exploded CAD 
view of the new five-spoke Wheg.

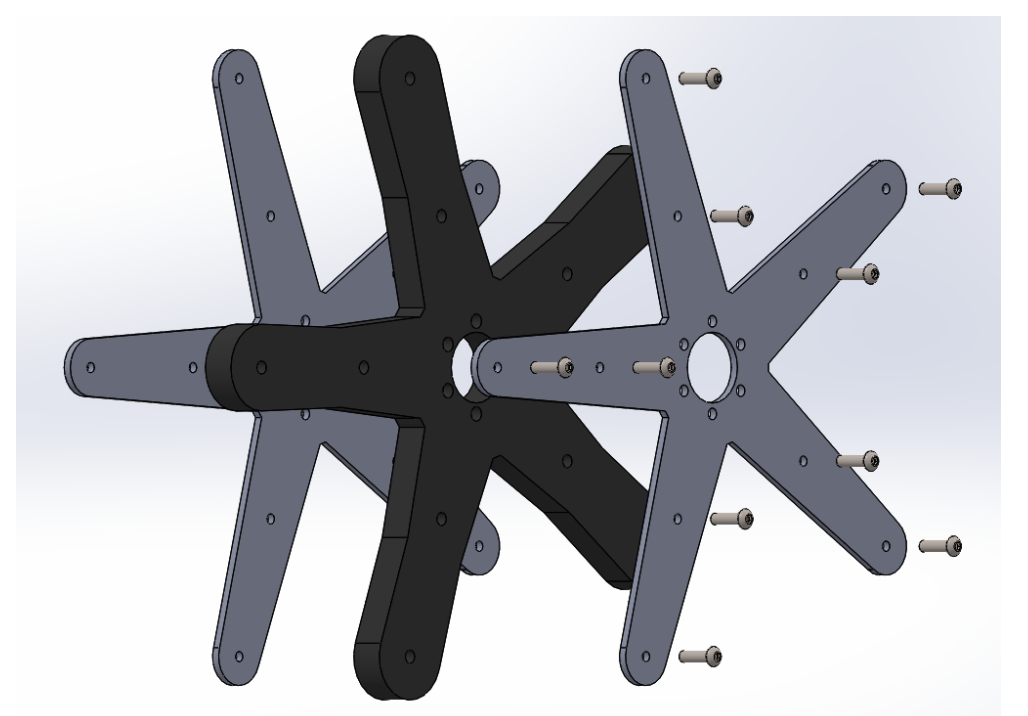

Figure 4.3: An exploded view of the five spoke Wheg assembly.

Unfortunately, due to the shortage of available $25 \mathrm{~mm}$ rubber sheet, the Wheg's diameter had to be reduced from $570 \mathrm{~mm}$ to $490 \mathrm{~mm}$. The six-spoke Whegs could also not be produced, as they did not fit on the available rubber sheeting. Thus, four of the five-spoke Whegs were produced, with a diameter of $490 \mathrm{~mm}$. The reduced diameter still meets the ground clearance requirement, however, the system is $40 \mathrm{~mm}$ lower than initially designed and therefore it is at risk of bottoming out over large obstacles. Removing the six-spoke Whegs from the rear axles will also re-introduce the vertical oscillations seen in the initial testing. However, the quick change Wheg mounting system will allow replacement Whegs to be quickly fitted at a later date.

For quick and accurate manufacture, both the rubber and the aluminium sheets were cut with a CNC water-jet. The bolt holes in the inner aluminium support were cut at $6.8 \mathrm{~mm}$ so they could be tapped to accept a M8 bolt. The bolts pass through the outer aluminium support and rubber and are threaded into the inner support. To prevent the bolts from un-threading during opera- 
tion, Loctite ${ }^{\mathrm{TM}}$ thread locker was added to each bolt. To prevent the sharp edges of the aluminium supports from damaging the rubber, all the edges were bevelled with a file.

\subsubsection{Wheel Design Summary}

The finished Whegs have a diameter of $490 \mathrm{~mm}$ and are made from $25 \mathrm{~mm}$ fibre reinforced rubber. The rubber is additionally strengthened with two $6 \mathrm{~mm}$ aluminium sheets clamped either side of the rubber with 18 M8 bolts. The rubber of each spoke of the Whegs is rounded to dampen the impact of the spoke hitting the ground. Figure 4.4 shows four of the Whegs mounted to HADES.

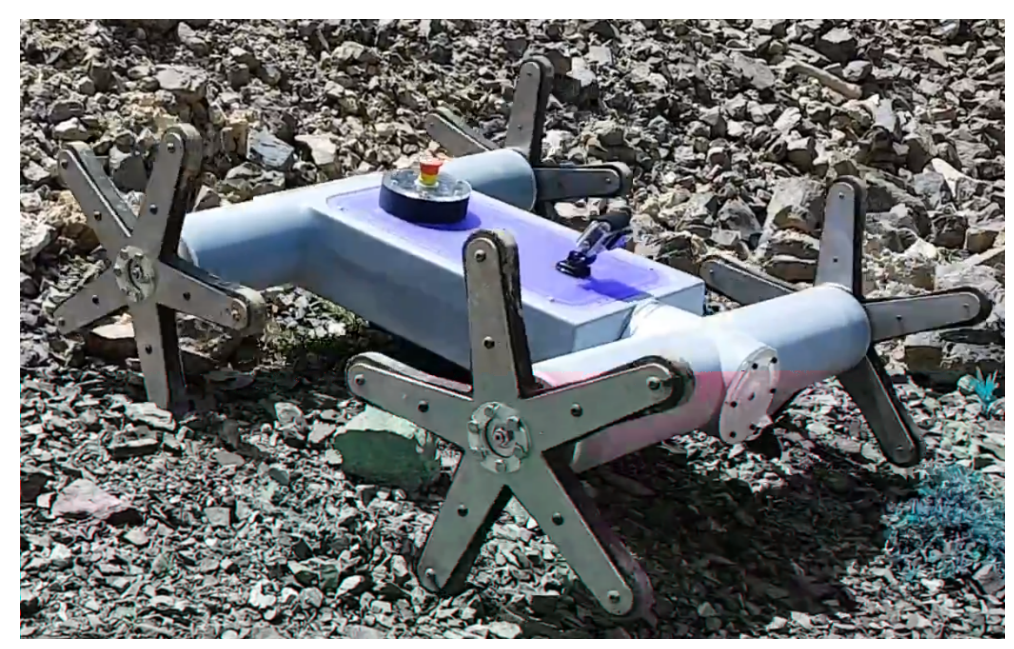

Figure 4.4: HADES moving on loose gravel with the $490 \mathrm{~mm}$ Whegs.

\subsection{Drive}

HADES should have an operational cruising speed of $1.4 \mathrm{~ms}^{-1}$ as per the requirements outlined in Section 2.4. Additionally, the drive system needs to provide sufficient torque for the Whegs to climb uneven and steep terrain. The 
drive systems used should be lightweight, and as low power as possible to extend the operational run-time of HADES. Four drive systems need to be implemented, one for each Wheg. The rotational speed of the drive shaft can be calculated from the Wheg diameter and the linear speed with Eq. 4.1.

$$
\omega=\frac{2 \cdot V_{\text {lin }}}{D},
$$

where $V_{\text {lin }}$ is the linear speed, $D$ is the diameter of the Wheg and $\omega$ is the required angular velocity of the drive system. To reach a linear speed of $1.4 \mathrm{~ms}^{-1}$ with the $490 \mathrm{~mm}$ Whegs, the shaft needs to rotate at $5.7 \mathrm{rads}^{-1}$.

The drive system also needs to provide torque for the worst-case scenario, which would be HADES lifting itself over an obstacle with only one Wheg in contact with the ground. In this case, the mass of the chassis (30 kg) is being lifted against gravity about a pivot point with a radius (r) of $D / 2(245 \mathrm{~mm})$. The worst case torque can be calculated using Eq. 4.2.

$$
\tau=m g r
$$

where $\tau$ is the required torque, $g$ is the acceleration due to gravity $\left(9.81 \mathrm{~ms}^{-2}\right)$ and $m$ is the mass of the chassis. Using Eq. 4.2 the maximum torque is calculated to be $72.1 \mathrm{Nm}$. In normal operation, the torque requirement will be much lower as the there will four Whegs in traction, and the chassis will not be climbing directly against gravity.

The speed and torque values were used to determine the gearbox and motor selection. In addition, to supplying the correct angular velocity and torque outputs, the drive systems need to be compact, lightweight and produce a minimum amount of excess heat.

\subsubsection{Gearbox}

Most small form factor DC motors are designed to deliver a high speed, low torque output, and therefore cannot provide the torque needed to meet the 
drive requirements. A reduction gearbox is needed to reduce the output speed and increase the torque. Planetary and strain wave gearboxes are the two most common gearbox configurations used in robotic applications [59]. Planetary gearboxes are cheaper and more common than strain wave gearboxes, however, they are larger for a given gear reduction and are less efficient.

Banebots P80 planetary gearboxes were selected due to their low cost and availability in low quantities. The P80 series of gearboxes are available in various reductions, ranging from 3:1 to 265:1. To meet the torque requirements, a 64:1 three-stage gearbox was selected. The $64: 1$ gearbox is rated to a maximum output torque of $115 \mathrm{Nm}$, which exceeds the $72.1 \mathrm{Nm}$ requirement for the drive system.

After the gearbox reduction, to supply torque for the worst case scenario, the motor will need to supply $1.12 \mathrm{Nm}$ of torque to the input sun gear of the gearbox. To meet the speed requirement, the input of the gearbox will need to be driven at $364.8 \mathrm{rads}^{-1}$.

\subsubsection{Motors}

A motor is needed to drive the input pinion of the gearbox to supply torque to the Wheg for movement. Continuous rotation DC electric motors are divided into two broad categories, brushed motors and brushless motors. Brushed motors are cheaper than brushless motors, however, brushless motors are usually lighter and more efficient. As brushless motors are more efficient, they do not produce as much heat energy, and do not spark in normal operation, unlike brushed motors. Eliminating sparking components is critical for any device that is operating in a potentially explosive atmosphere [11]. For these reasons, brushless motors are used in HADES. The torque $(\tau)$ and output speed $(\omega)$ of a brushless motor for a supplied current $(I)$ and voltage $(V)$ is given by Eq. 4.3 and 4.4 . 


$$
\begin{gathered}
\tau=I \cdot \frac{1}{K_{v}} \\
\omega=V \cdot K_{v}
\end{gathered}
$$

where $K_{v}$ is the motor speed constant expressed in $\mathrm{rads}^{-1} \mathrm{~V}^{-1}$. The HADES motor needs to supply high torque and relatively low angular velocity, and for this reason, a motor with a low $K_{v}$ value is needed. To meet this requirement, the Hobbystar 56112 brushless in-runner motor is selected. The Hobbystar motor has a $K_{v}$ of $33.5 \mathrm{rads}^{-1} \mathrm{~V}^{-1}$ with a maximum current and voltage of $180 \mathrm{~A}$ and $53 \mathrm{~V}$ respectively. Thus, the Hobbystar motor can supply a maximum torque of $5.4 \mathrm{Nm}$ and a maximum angular velocity of $1775.5 \mathrm{rads}^{-1}$. Both of these numbers are well in excess of the specifications needed for the drive systems.

The Banebots P80 gearboxes do not natively support mounting to the Hobbystar 56112 motors. A bespoke mounting system is needed to mount the motor concentrically to the first sun gear in the planetary gearbox. To connect the motor to the gearbox, a custom mounting block was machined from aluminium. The block can be seen in Figgure 4.5.

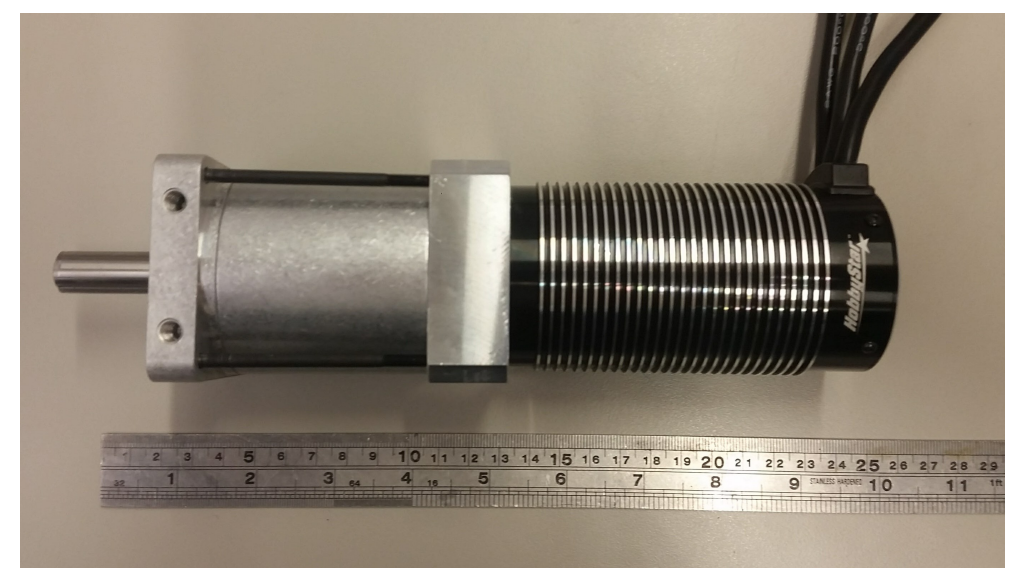

Figure 4.5: The Hobbystar 56112 brushless motor and the Banebots P80 64:1 gear connected together with a bespoke mounting block. 


\subsection{Wheg and Chassis Support}

The motor and gearbox need to be securely mounted to the chassis. The Wheg also needs to be mounted to the output shaft from the motor drive system. The Banebots' gearboxes only have a single output bearing and cannot support large radial and axial loads on their output shafts. The drive gearbox mounting system will need to add support bearings to the gearbox output shaft to support the large impacting loads from the Whegs. The gearbox mounting system will also need to seal against water and dust ingress to meet the IP68 standard.

A robust mounting system is needed for coupling the Wheg to the output shaft of the gear. To allow the Wheg to be changed or removed for transport, the mounting system needs to allow for easy removal/attachment of the Wheg. The Wheg mounting system also needs to be able to withstand the torque and axial forces associated with operating HADES.

To meet the A/NZ60079.11 positive pressure system standard, the chassis must be able to fall from $1 \mathrm{~m}$ and still be able to maintain a gas seal [11]. The average force $\left(F_{a}\right)$ needed to stop a falling object is given by Eq. 4.5.

$$
F_{a}=\frac{m g h}{d_{\text {stop }}}
$$

where $m$ is the mass of the object, $g$ is the acceleration due to gravity $\left(9.81 \mathrm{~ms}^{-2}\right)$, $h$ height is the height the object is falling from and $d_{\text {stop }}$ is the stopping distance. For HADES (30 kg) falling from a height of $1 \mathrm{~m}$ and stopping in $50 \mathrm{~mm}$, the average stopping force is $8820 \mathrm{~N}$. Assuming HADES lands on at least two Whegs, the maximum force per drive shaft is $4410 \mathrm{~N}$. 


\subsubsection{Revision One}

To minimise cost and engineering effort, the initial design of the drive system support used as many off-the-shelf components as possible. BaneBots offer extended shafts as an extra for their gearboxes, the $82.55 \mathrm{~mm}\left(3.25^{\prime \prime}\right)$ long versions of these shafts were purchased for integration into the drive system.

To meet the requirements, the output shaft needs to transmit $72.1 \mathrm{Nm}$ of torque to the Wheg. The BaneBots' shafts are made from AISI 4140 high tensile steel and have a diameter of $12.7 \mathrm{~mm}\left(0.5^{\prime \prime}\right)$. There is a $3.175 \mathrm{~mm}\left(0.125^{\prime \prime}\right)$ by $19 \mathrm{~mm}\left(0.75^{\prime \prime}\right)$ keyway in the shaft. The maximum shear torque of the shaft with a keyway is given by Eq. 4.6.

$$
\tau=\frac{T d L h}{2}
$$

where $\tau$ is the maximum shear torque of the shaft, $T$ is the yield stress of the shaft/key, $d$ is the diameter of the shaft and $L$ and $h$ are the length and depth of the keyway respectively. For the 4140 steel shaft, the yield strength is $415 \times 10^{6} \mathrm{Nm}^{-2}$, which results in maximum shear torque of $158.9 \mathrm{Nm}$. This is more than double the expected torque, and therefore the BaneBots' shaft meets the torque requirements.

To meet the design requirements, the shaft support needs to be able to withstand a maximum impact load of 4410 N. Two R8-2Z deep groove ball bearings are used to support the shaft, these are spaced apart by $28.575 \mathrm{~mm}\left(1.125^{\prime \prime}\right)$; the first bearing is located $22.225 \mathrm{~mm}\left(0.875^{\prime \prime}\right)$ from the load point of the shaft. The R8-2Z bearings have a dynamic load rating of $4.42 \mathrm{kN}$. Equation 4.7 shows the calculation for bearing load for an overhung shaft load.

$$
F_{b}=\frac{F_{l} L}{L_{1}-L_{2}}
$$


where $F_{b}$ is the force on the bearing, $F_{s}$ is the force on the shaft, $L$ is the distance from the load to the bearing, $L_{1}$ is the distance from the load to the inner bearing $(50.8 \mathrm{~mm})$ and $L_{2}$ is the distance from the load to the outer bearing $(22.225 \mathrm{~mm})$. When the maximum force on the shaft is $4410 \mathrm{~N}$, the outer and inner bearings have maximum loads of $7.84 \mathrm{kN}$ and $3.4 \mathrm{kN}$ respectively. This exceeds the dynamic load rating of the outer bearing. However, stronger $12.7 \mathrm{~mm}$ bearings are considerably more expensive and harder to source. As this drive mounting system is being developed to quickly test HADES' locomotion capabilities it is unlikely to experience any large shock loads. Once validation of the drive system is complete, a second revision of the bearing block is developed.

A bearing block is needed to hold the bearings and gearbox in position. The bearing block will need to mount to the chassis using the six bolt holes covered in Section 3.1.3. To prevent water ingress, the bearing block will also need to seal the rotating shaft and chassis mount to the IP68 standard.

To mount the gearbox to the bearing block, the four 1/4-20 tapped holes on the side of the gearbox front plate are used. A square front plate aligns with two bosses on the rear of the bearing block; these centre the gearbox over the bearings. Figure 4.6 shows the bearing block mounted to the drive system.

To seal the bearing block to the chassis, a $98 \mathrm{~mm}$ ID, $3 \mathrm{~mm}$ profile O-ring is used. This O-ring mounts in a $3.4 \mathrm{~mm}$ wide by $2 \mathrm{~mm}$ deep groove on the back of the mounting flange. The groove is sized such that the O-ring protrudes forward of the face and has enough room to compress, forming a seal. The empty O-ring groove can be seen in Figure. 4.6. The spinning shaft is sealed with a OS.050112 low-pressure lip seal, this can be seen in Figure 4.7.

The bearings mount through a bore in the centre of the bearing block. A journal in the centre of the block is used to set the spacing. The bore is toleranced such that the bearings are retained with a press-fit. Figure 4.7 shows a cross-section of the bearing block. 


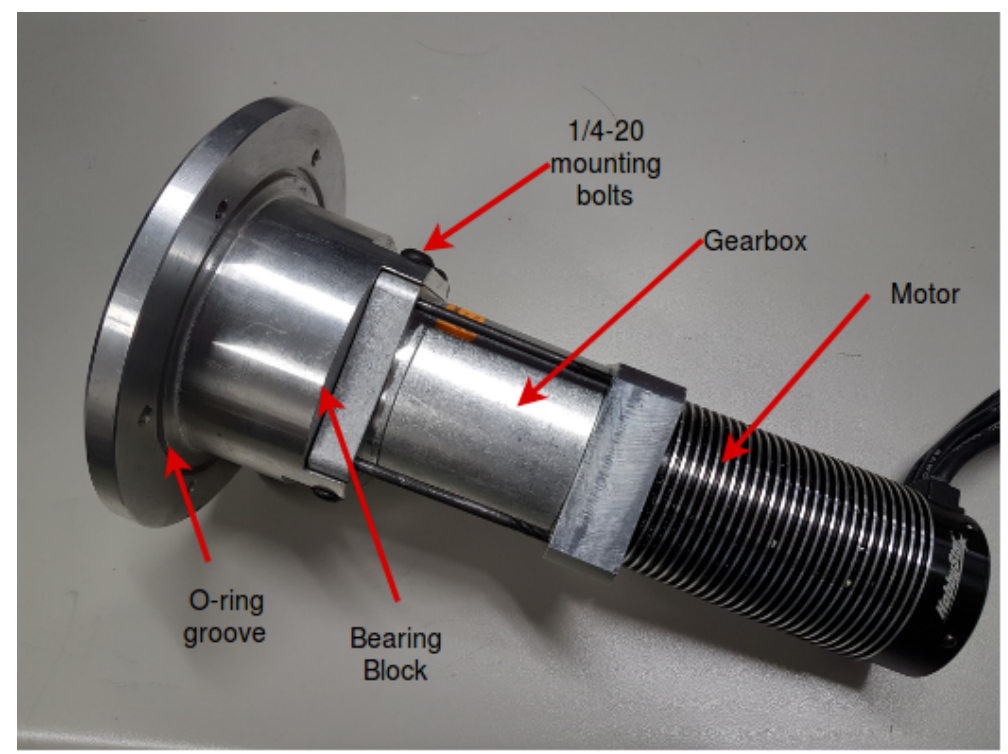

Figure 4.6: The revision one gearbox-bearing block mount.

During initial trials and assembly of the drive system mount, some problems were discovered. The square front face of the gearbox is made from cast aluminium and is not machined to a precise size. Thus, in some cases, when the bolts holding the gearbox are tightened, the gearbox is pulled out of alignment with the bearings in the bearing block. Misalignment of the bearings and the shaft causes the gearbox to wear unevenly and in one case caused the gearbox to bind.

Removing and mounting the Whegs to/from the shafts also proved problematic due to the different grades of material in the mounting system. The shafts are made from high tensile steel, the keys from 1018 mild steel and the hubs from 2014 free machining aluminium. The 2014 aluminium is much softer than the two steels used. Thus, when the system was under load, the aluminium would deform and bind to the key and the shaft, making the Wheg extremely difficult to remove. Additional to the problems found in the testing, the shaft bearings cannot survive the $1 \mathrm{~m}$ fall that is necessary for the A/NZ60079.29 standard. The bearings in the bearing block are not actively 


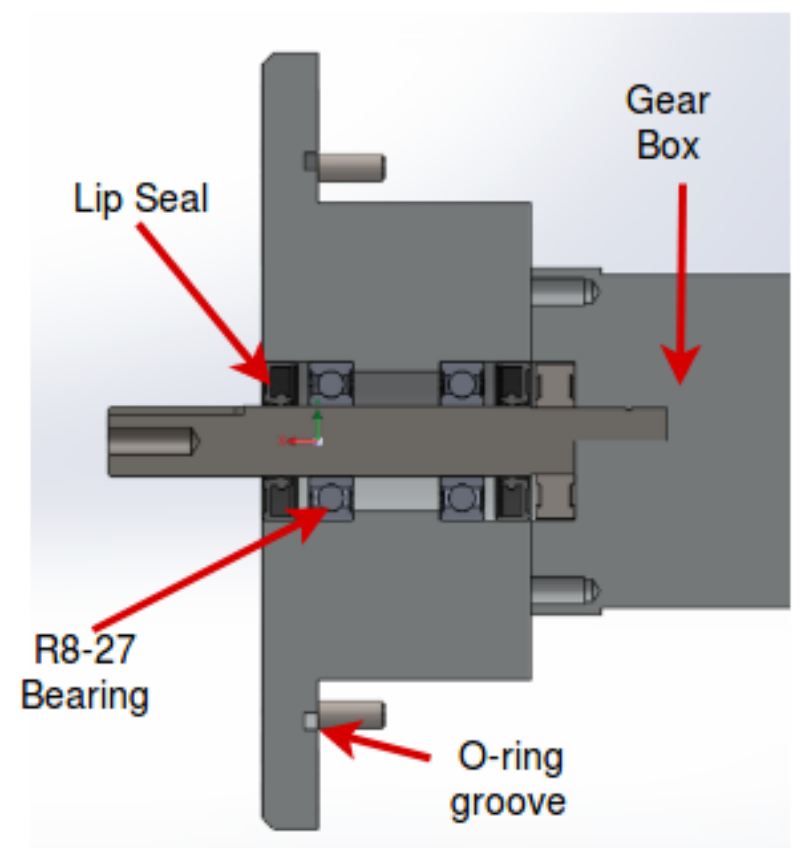

Figure 4.7: A cross section view of the revision one bearing block and gearbox support.

retained, relying only on a press-fit. For high vibration applications, it is recommended that bearings/shafts are retained with an active retention method [70].

\subsubsection{Revision Two}

Revision two improved on revision one by mitigating the gearbox alignment issue, increasing the shaft support strength, implementing an easier to use method of Wheg mounting and improving bearing retention.

The gearbox shaft binds when the gearbox and bearing supports are not concentric. To ensure that the gearbox and bearings remain concentric, a bespoke front end for the gearboxes was developed. The new front end replaces the square front end supplied with the gearboxes. It has a circular boss that lo- 
cates in a bore on the bearing block to ensure accurate alignment. The circular boss also locates and axially secures the inner shaft support bearing. The boss is sized so that it only clamps on the outer race of the bearing, preventing the bearing from binding.

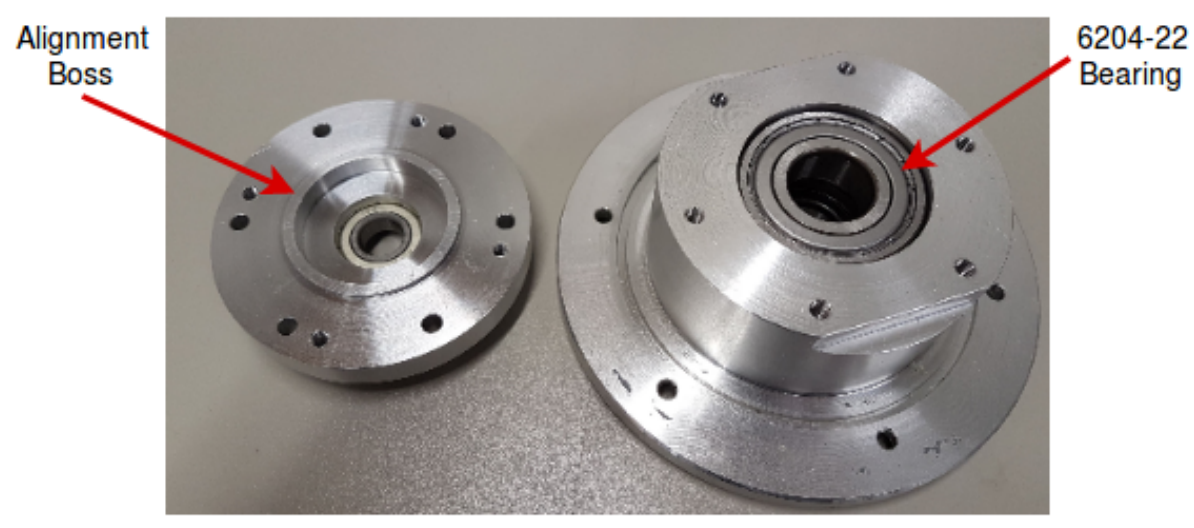

Figure 4.8: The disassembled gearbox front end (left) and bearing block (right), showing the alignment boss on the gear box front end.

To keep the weight down and allow for easy machining, the new front end is made from 2011 free machining grade aluminium. The new front end needs to align with the outer barrel/ring gear of the gearboxes. The gearbox barrels are aligned with four $2 \mathrm{~mm}$ pins on a $55 \mathrm{~mm}$ pitch circle diameter (PCD). As aluminium and mild steel pins would sheer under the torque from the drive motors, hardened steel pins are pressed into the aluminium front end. To support the shaft as it enters the gearbox, a $6001-\mathrm{ZZ} 12 \mathrm{~mm}$ bearing is mounted inside the new front plate. Figure 4.8 shows the disassembled gearbox - bearing block mount, where the circular alignment boss can be seen on the left of the figure.

To secure the gearbox together, four M5 lag bolts secure the motor mount to the front plate. The gearbox front end is mounted to the bearing block with six M5 bolts in a $72 \mathrm{~mm}$ PCD pattern. To increase the nominal strength of the shaft, its diameter is increased from $12.7 \mathrm{~mm}$ to $20 \mathrm{~mm}$. 
To support the larger shaft size, two 6204-ZZ $20 \mathrm{~mm}$ ID bearings are mounted inside the bearing block. The larger bearings have a larger dynamic load rating of $13.5 \mathrm{kN}$, thus they will survive the $4.41 \mathrm{kN}$ impact load when HADES falls from $1 \mathrm{~m}$. The revision one shaft was axially retained in the bearing block with a $12 \mathrm{~mm}$ circlip. The front bearing also relied on a press-fit to prevent it from falling out from the bearing block.

To retain the new shaft in the bearing block, two circlips are used, one on the $12 \mathrm{~mm}$ section and one on the $20 \mathrm{~mm}$ section. The front bearing is retained with a $3 \mathrm{~mm}$ journal on the shaft. To transmit the required torque, the new shaft is constructed from AISI 4140 high tensile steel. Figure 4.9 shows a cross-section view of the gearbox front end, shaft and shaft support bearings.

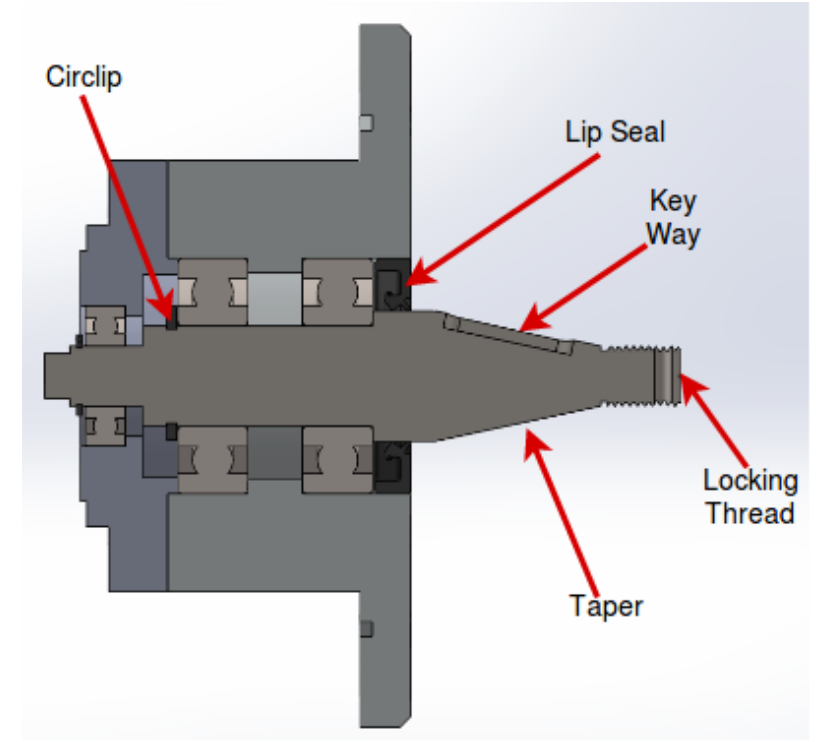

Figure 4.9: The revision two gearbox front end, bearing block and shaft.

To improve the repeatability and ease of use for the Wheg mounting system, a bespoke tapered fitting was implemented. A tapered fitting consists of a male and female taper that are compressed inside one another allowing for torque transfer. Tapered fittings are typically compressed together with a form of threaded fastener, however, some are permanently bonded with a hydraulic 
press. Tapered couplings provide accurate radial positioning and when designed correctly, they have high torque transfer properties. If the tapered fit alone cannot transfer the necessary torque, a key can be added to the taper. Adding a key also increases the reliability of the taper as all the torque transfer is not dependent on the taper clamping force.

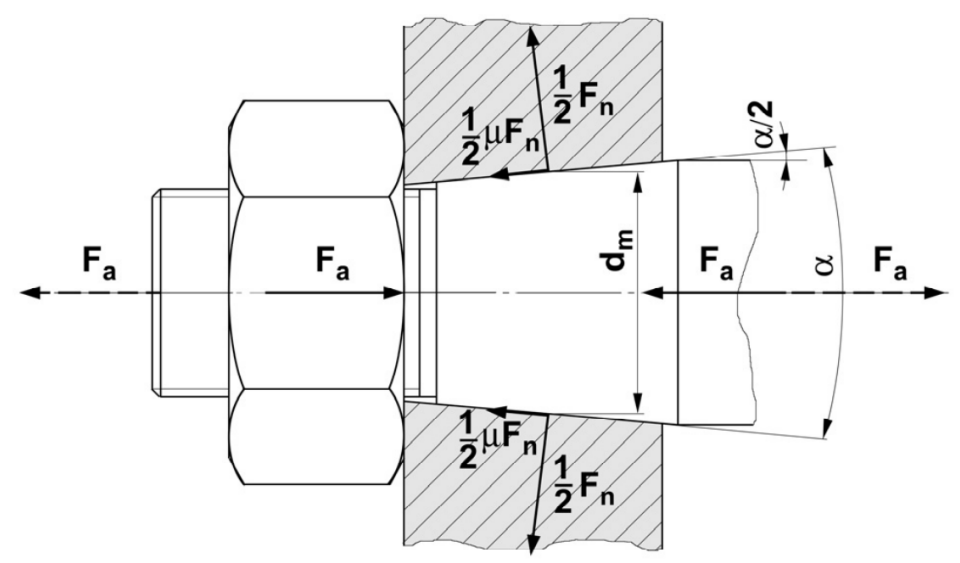

Figure 4.10: The complete force diagram for a taper system compressed with a nut [25]

Tapers rely on the interference fit between the two parts for power transmission. The transmitted torque is dependant on several factors, such as: taper angle, taper material, compression force and the su rface finish of the tapered parts. For use in HADES, the tapered fitting must be able to transmit $72.1 \mathrm{Nm}$ of torque. The torque is a function of the clamping force between the two tapered surfaces, the median diameter of the taper and coefficient of friction between the two surfaces. The relationship is given in Eq. 4.8.

$$
\tau=\mu \cdot F_{n} \frac{d_{m}}{2},
$$

where $\tau$ is the output torque, $F_{n}$ is the clamping force normal to the taper, $\mu$ is the coefficient of friction between the two surfaces and $d_{m}$ is the median diameter of the taper. When a threaded fastener supplies the clamping force holding the tapers together, the normal force $F_{n}$ can be given by Equation 4.9 . 


$$
F_{n}=\frac{\frac{\tau_{n}}{K d}}{\sin \frac{\alpha}{2}+\mu \cos \frac{\alpha}{2}}
$$

where $\tau_{n}$ is the torque applied to the threaded fastener, $d$ is the diameter of the fastener, $K$ is the lubrication coefficient of the fastener and $\alpha$ is the included angle of the taper. To supply the clamping pressure for the taper an M12 castellated nut is used; to retain the nut on the shaft, a split pin is used. A thread cut in AISI 4140 steel is equivalent to a grade 10.9 bolt, when tightened to the correct torque $(65.1 \mathrm{Nm})$ an M12 thread can supply $27.1 \mathrm{kN}$ of clamping pressure [70]. To meet the torque requirements at a median diameter of $19 \mathrm{~mm}$, a taper angle of $24^{\circ}$ was calculated.

To increase the safety factor and prevent damage if the nut did work loose, a $6 \mathrm{~mm}$ key was added to the taper. The taper and key mate with a tapered hub that then mounts to the Wheg. The tapered hub needs to be made from a hard, but lightweight material to prevent the taper from deforming under the clamping pressure. To meet these criteria, the tapered hub is made from 7075-grade aluminium, which was chosen as it is the hardest grade of commonly available aluminium. To attach the Wheg to the hub, eight M8 threaded holes are used. The tapered hub also has a circular boss for aligning the Wheg concentrically. Figure 4.11 shows the hub mounted to the taper on the front of the breaking block.

\subsection{Drive System Summary}

Four locomotion systems are used to allow HADES to climb the varying terrain that are found in underground mines. The drive torque for each system is supplied with a Hobbystar 56112 planetary gearbox and a BaneBots 64:1 planetary gearbox. The output shafts from the gearboxes cannot natively support the axial and radial loads needed for HADES. To support these loads and mount the drive systems into the chassis, a bespoke bearing block and shafting system 


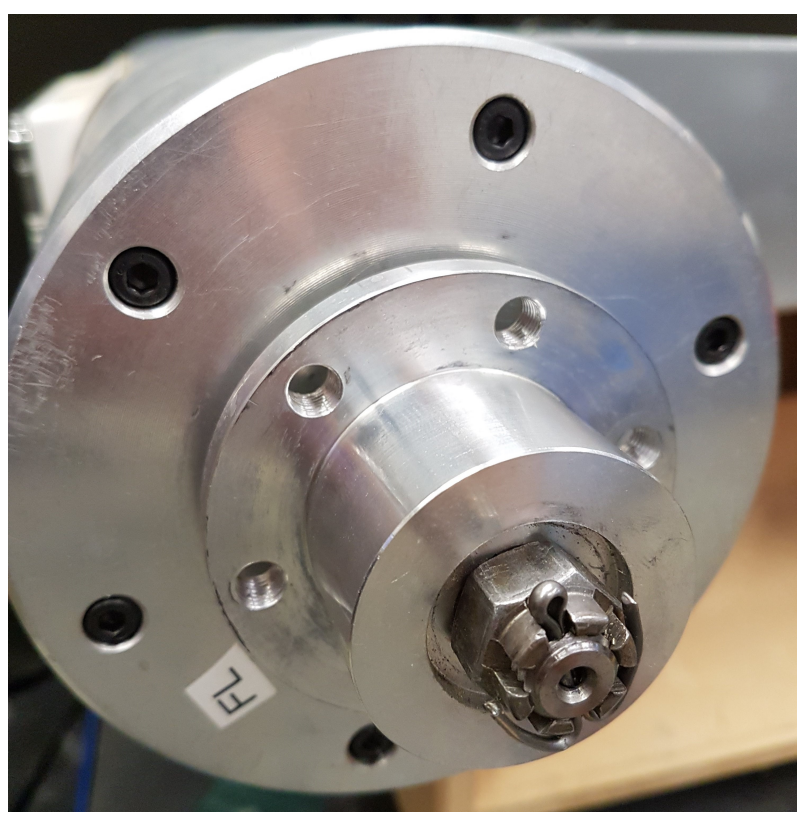

Figure 4.11: The tapered hub mounted to the shaft and drive system.

was developed. The shafting system mates with a tapered hub to transfer the torque to the Whegs. 


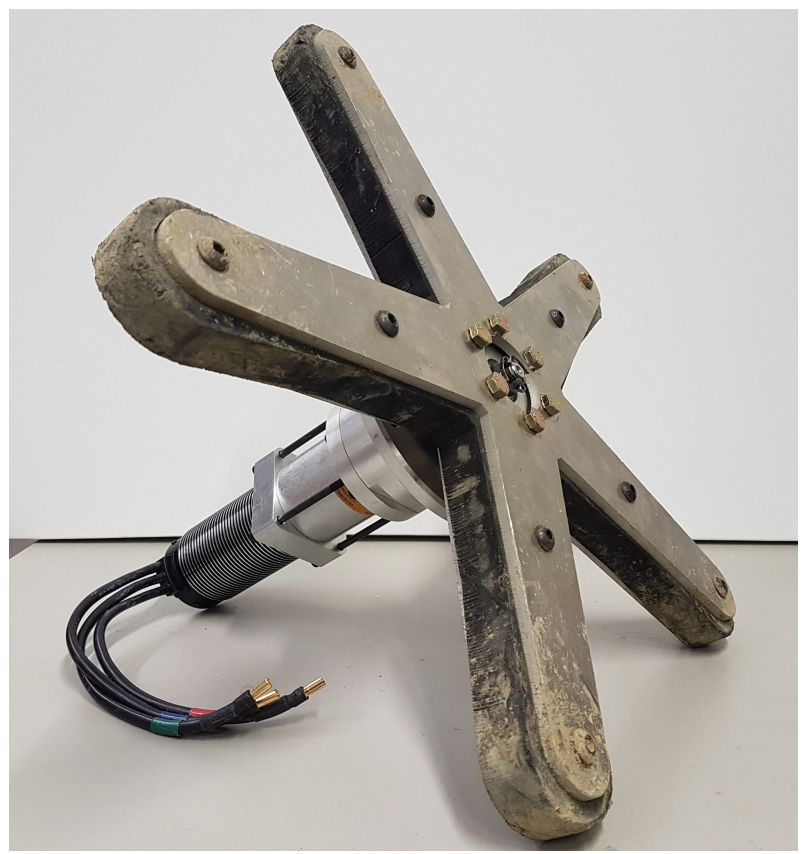

Figure 4.12: The complete locomotion system when it is not mounted inside HADES. 


\section{Chapter 5}

\section{Electronics - Sensor Systems}

\subsection{Overview}

As detailed in Chapter 1, the main purpose of sending HADES into an underground mine post-disaster is to locate survivors and ascertain the condition of the mine. To achieve this, HADES will need to carry a comprehensive set of atmospheric, visual and localisation sensors.

The sensors represent two sub-systems of the HADES system, the environmental sensors and the camera system. To prevent a fault in any sub-system from cascading to the rest of the system, the modules are implemented as independent systems (Section 2.4). The complete system diagram of HADES' electronics can be seen in Figure. 5.1. This chapter covers the implementation of the two green blocks; the sensor and camera systems, the implementation of the other sub-systems are discussed in Chapter 6. 


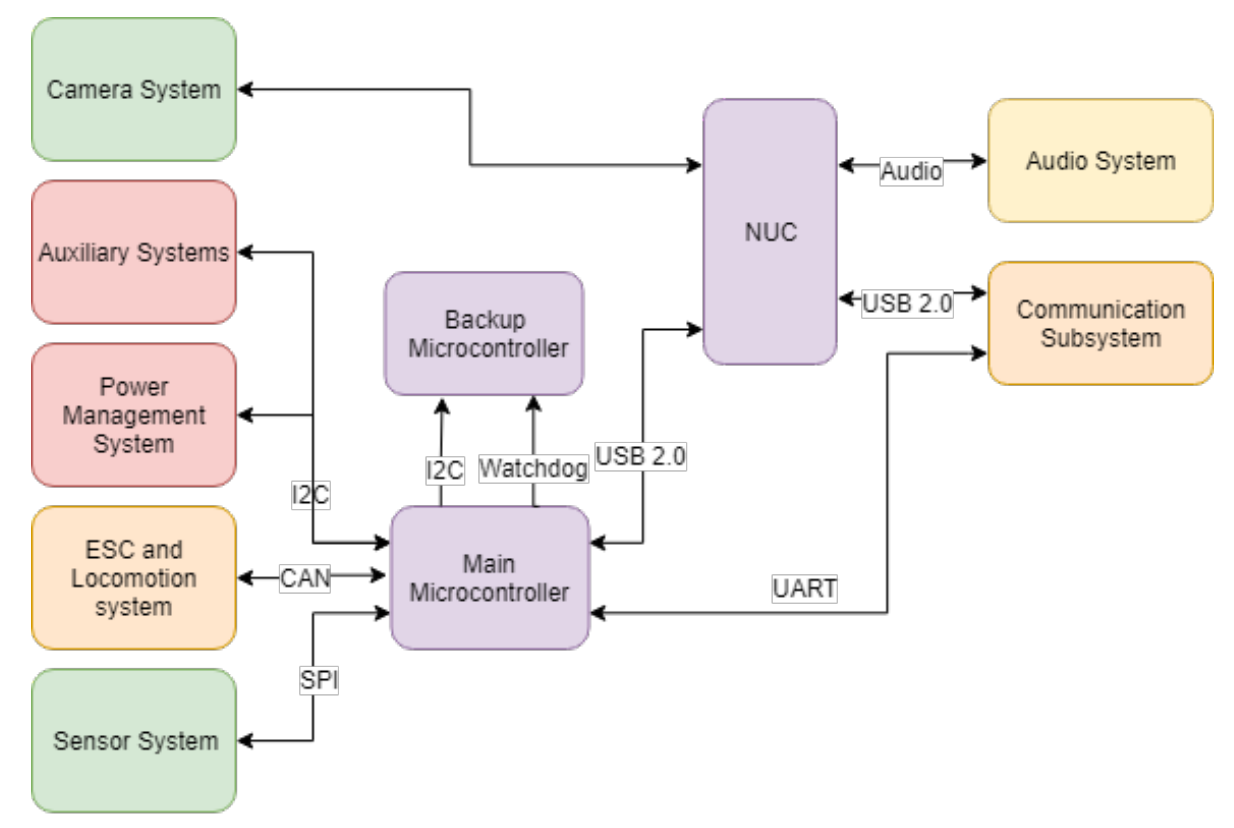

Figure 5.1: The top level block diagram for the electronics system in HADES

\subsection{Environmental Sensors}

As discussed in Section 2.1.2 underground mines often have high concentrations of toxic and explosive gasses. Before any manned rescue attempts can be made, an assessment of these gas levels is needed. Rescue teams will also benefit from an assessment of the temperature, humidity and barometric pressure. Knowledge of the barometric pressure is also needed for the positive pressure explosion prevention system (Section 5.2.3.2).

The environmental sensor system consists of two sub-modules, the internal and external sensors. As defined in in Figure 5.1, to integrate with the overall electronics system, the sensor system should have an SPI bus interface. Figure 5.2 shows a block diagram of the sensor system. 


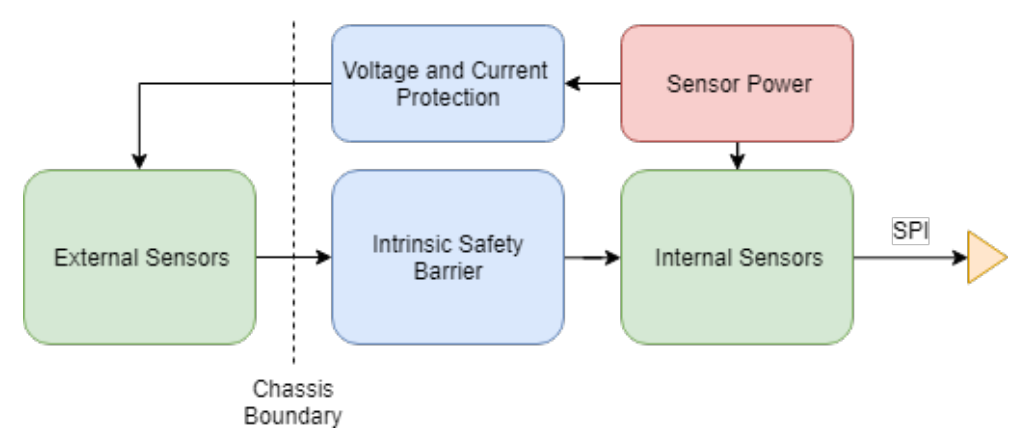

Figure 5.2: The block diagram for the HADES sensor system

\subsubsection{External Sensors}

The external sensors on HADES are sensors that are not contained within the chassis. These sensors are used for monitoring the gas concentrations, temperature, humidity and pressure of the atmosphere surrounding the chassis. As these sensors are in contact with a potentially explosive atmosphere, they will need to meet the intrinsic safety requirements outlined in A/NZ60079.11, see Section 5.2.2 [11].

\subsubsection{Gas Concentration Sensors}

As discussed in Section 2.1.2 the New Zealand Government prescribes maximum concentrations of gasses that are acceptable for human entry, and this informs the selection of appropriate sensors, specifically their precision and range. Gas concentrations sensors are grouped into three categories: electrochemical sensors, IR sensors and spectrometers. Initial investigation of IR and spectrometry sensors revealed that they are expensive and large devices, often only capable of measuring one or two gasses. The cost and size of electrochemical sensors make them much more suitable for use in a compact, low-cost robotic system such as HADES. 
Electrochemical sensors work by reducing or oxidising the target gas to produce a current proportional to the gas concentration. In most cases, a catalyst is used to assist the reaction, and in some cases the reaction chamber must be heated. Electrochemical sensors have a limited lifespan as the chemical reaction will eventually corrode the sensor electrodes. Operational life typically ranges from six months to three years, depending on the sensors and the environment.

To produce accurate gas readings, the sensors need to be regularly calibrated against known gas concentrations. Each manufacturer typically specifies a recommended recalibration period; the New Zealand Government mandates that any gas sensor needs to be calibrated at least annually [12].

City Technology and Alpahsense sensors are used in HADES due to their low cost, ease of purchase in low quantities and good supporting documentation. Where possible, the preference is for Alphasense sensors as they already meet numerous international intrinsic safety standards. Using seven sensors, seven out of the eight target gasses (Section 2.1.2) can be sampled. Nitrogen is not sampled, as with sufficient oxygen it is not considered toxic and it is an inert gas [1]. Table 5.1 outlines the target gases and the associated sensors.

Table 5.1: Target Gasses and their associated sensors.

\begin{tabular}{|l|l|l|}
\hline Gas (Symbol) & Sensor & Range \\
\hline Oxygen $\left(\mathrm{O}_{2}\right)$ & City Technology ME2-02 [34] & $0-25 \%$ \\
\hline Methane $\left(\mathrm{CH}_{4}\right)$ & City Technology 4P-50 [23] & $0-120 \%$ LEL $^{1}$ \\
\hline Hydrogen $\left(\mathrm{H}_{2}\right)$ & City Technology 4P-50 & $0-120 \%$ LEL \\
\hline Carbon Dioxide $\left(\mathrm{CO}_{2}\right)$ & City Technology IRcell - CO2 [22] & $0-10 \%$ \\
\hline Carbon Monoxide $(\mathrm{CO})$ & Alphasense CO-A4 [7] & $0-2000$ ppm \\
\hline Hydrogen Sulfide $\left(\mathrm{H}_{2} \mathrm{~S}\right)$ & Alphasense H2S-A4 [6] & $0-100$ ppm \\
\hline Sulfur Dioxide $\left(\mathrm{SO}_{2}\right)$ & Alphasense SO2-A4 [5] & $0-100$ ppm \\
\hline Nitrogen $\left(\mathrm{N}_{2}\right)$ & - & - \\
\hline
\end{tabular}

${ }^{1}$ Lower Explosive Limit 
The Alphasense A4 sensors are four electrode electrochemical cells, used to sample $\mathrm{H}_{2} \mathrm{~S}, \mathrm{SO}_{2}$ and $\mathrm{CO}$. The sensor works by reducing or oxidising the target gas between the working and counter electrodes. The reference electrode is used to track the potential of the working electrode so current can be supplied to the counter electrode. The auxiliary electrode is used to remove temperature and other offsets from the working electrode. The A4 sensors have sensitivities in the 100 to $1500 \mathrm{nA} / \mathrm{ppm}$ range and therefore to accurately sense these small currents supporting, circuitry is needed.

A potentiostatic circuit is used to control and read the four electrode sensors. This circuit is responsible for balancing the current in the working and counter electrodes, maintaining a minimum potential difference between the working and reference electrodes and amplifying the output currents to a value detectable by an ADC.

Op-amp U1A (Figure 5.3) is configured as an inverting amplifier between the counter and reference electrodes. As the working electrode uses more current, the potential of the reference electrode lowers thus raising the potential of the counter electrode allowing for more current flow. Circuit stability and noise control are completed with R1, R2, C1 and C2 acting as filters to prevent oscillation and remove noise. $\mathrm{R} 1$ and $\mathrm{R} 2$ also prevent the op-amp from sinking over $5 \mu \mathrm{A}$ from the reference electrode (as specified in the sensor data sheet [8]).

As the concentration of the gas changes, the working electrode will sink or source more current. U1B (Figure. 5.3) is configured as a trans-impedance amplifier. The sensor current is reflected across R5, generating a voltage relative to ground. The gain of the circuit is set by $\mathrm{R} 4$, with $\mathrm{C} 5$ reducing high-frequency noise and preventing oscillation. U1 was chosen for its ability to sink and source similar amounts of current and for its high current output that is needed to drive the counter electrode.

The auxiliary electrode functions identically to the working electrode, ex- 


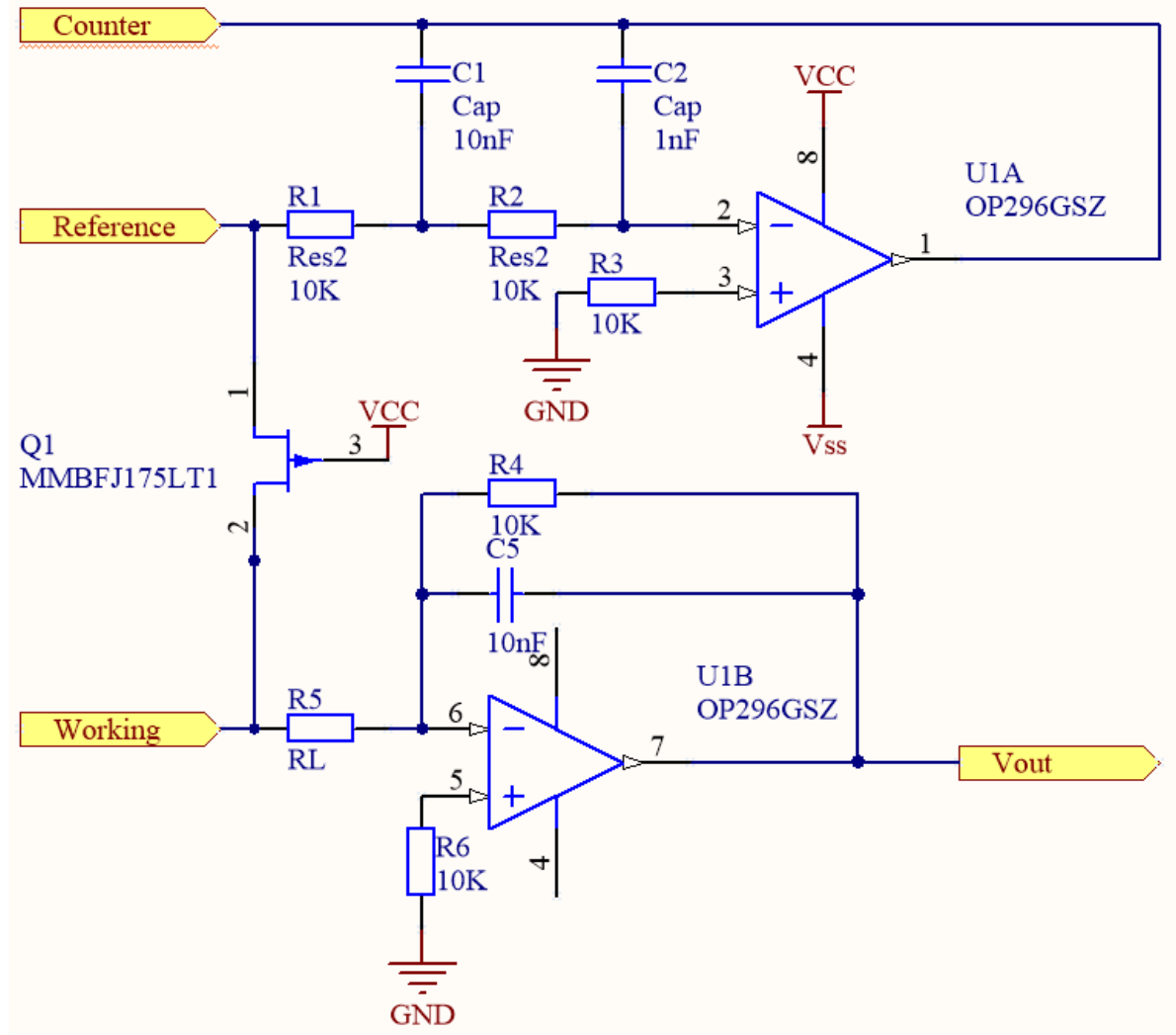

Figure 5.3: The circuity for the counter, reference and working electrodes of an A4 series gas sensor

cept the current does not vary with gas concentration. This means the output current can be used for off-set nulling the current from the working electrode. To simplify the software conversion, the same trans-impedance amplifier configuration is used as the working electrode, as shown in Figure. 5.4.

When the circuit is not powered, it is recommended that the auxiliary and working electrodes are shorted to the reference electrode. Connecting the three electrodes in an unpowered state also reduces the sensor start time by 1-4 minutes. To short the electrodes, a depletion mode P-MOS transistor is used (Q1 and Q2 in Figure. 5.3 and Figure. 5.4). When power is applied, the transistor turns off and allows the reference electrode potential to be controlled by U1A. 


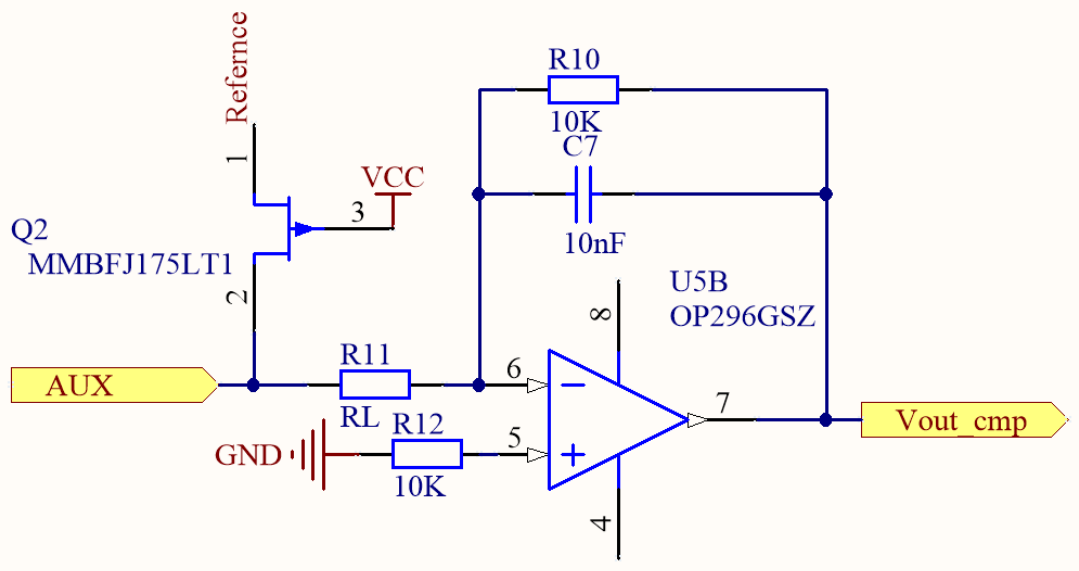

Figure 5.4: The circuity for the Auxiliary electrode of an A4 series gas sensor

When power is removed, the transistor begins conducting and connects the two electrodes.

The sensitivity and operating range of each potentiostatic circuit is set by $R_{L}$ and $\mathrm{R}_{G}$. The values of these resistors are chosen so that at full output current for each sensor the output voltage does not saturate the $\pm 5 \mathrm{~V}$ ADC input. Table 5.2 shows the resistor values used and the output voltage range.

Table 5.2: A4 Series sensor load and gain resistors.

\begin{tabular}{|l|l|l|l|l|l|l|}
\hline Sensor & Sensitivity & Full Scale & $\begin{array}{l}\text { Full Scale } \\
\text { Current }\end{array}$ & $\mathbf{R}_{L}$ & $\mathbf{R}_{G}$ & $\begin{array}{l}\text { Full scale } \\
\text { Voltage }\end{array}$ \\
\hline $\mathrm{CO}-\mathrm{A} 4$ & $375 \mathrm{nA} / \mathrm{ppm}$ & $2000 \mathrm{ppm}$ & $750 \mu \mathrm{A}$ & $100 \Omega$ & $5.6 \mathrm{k} \Omega$ & $4.2 \mathrm{~V}$ \\
\hline $\mathrm{H}_{2} \mathrm{~S}-\mathrm{A} 4$ & $1850 \mathrm{nA} / \mathrm{ppm}$ & $100 \mathrm{ppm}$ & $185 \mu \mathrm{A}$ & $91.5 \Omega$ & $24 \mathrm{k} \Omega$ & $4.44 \mathrm{~V}$ \\
\hline $\mathrm{SO}_{2}-\mathrm{A} 4$ & $480 \mathrm{nA} / \mathrm{ppm}$ & $100 \mathrm{ppm}$ & $48 \mu \mathrm{A}$ & $100 \Omega$ & $100 \mathrm{~K} \Omega$ & $4.8 \mathrm{~V}$ \\
\hline
\end{tabular}

City Technology sensors are used to measure the concentration of combustible gasses, oxygen and carbon dioxide surrounding the chassis. The oxygen sensor is a passive ME2-02 sensor, which produces a current proportional to the oxygen concentration [34]. The output current of the oxygen sensor is converted 
into a voltage using a trans-impedance amplifier (U3, Figure 5.5). The gain of the trans-impedance amplifier is set with $\mathrm{R}_{g}$ to ensure that the output uses the full range of the ADC. $R_{g}$ is calculated by,

$$
V_{o}=I_{s} R_{L}\left(1+\frac{R_{g}}{R_{L}}\right)
$$

where $I_{s}$ is the maximum current from the sensor, $R_{L}$ is the load resistor and $V_{o}$ is the output voltage. The maximum current from the ME2-02 is $0.250 \mathrm{~mA}$, when used with a $3.3 \mathrm{~V}$ ADC and an $100 \Omega$ load resistor; a gain resistor of $13 \mathrm{k} \Omega$ is needed.

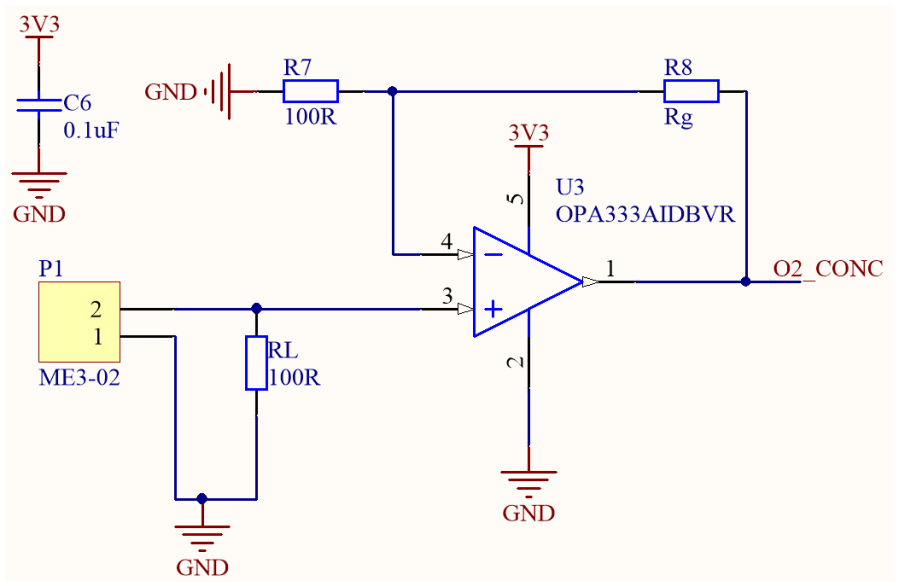

Figure 5.5: The support circuity for the ME2-02 oxygen gas sensor.

The City Technology 4P-50 is a pellistor gas sensor that can sense 17 different hydrocarbons (including methane) and hydrogen gas [23].

A pellistor consists of small pellets of loaded ceramics whose resistance changes in the presence of the target gas. To work correctly the pellets have to be heated. To ensure the heating element does not cause an explosion, a sintered mesh housing shields the sensor. The pellistor sensor is interfaced to the ADC with a Wheatstone bridge and a differential amplifier.

This sensor is used in a half bridge configuration, where the sensor is one half of the bridge. Resistors R1, R2 and R3 (Figure 5.6) are the remaining half of 


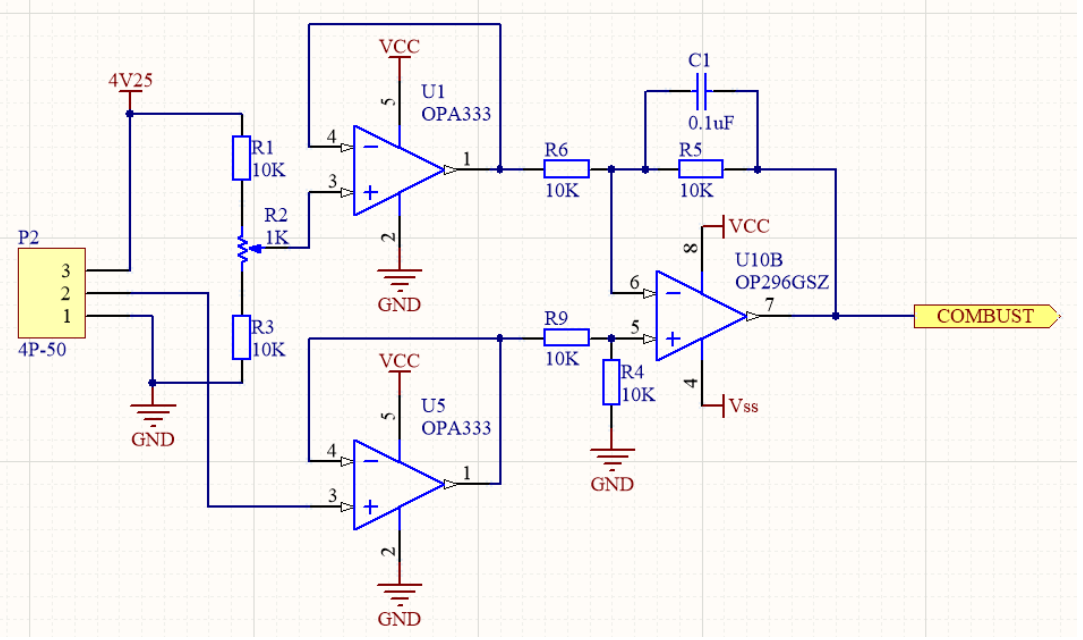

Figure 5.6: The support circuity for the 4P-50 combustible gas sensor.

the Wheatstone bridge. To set the zero point, $\mathrm{R} 2$ is variable. To isolate the input impedance of the differential amplifier from the Wheatstone bridge, U1 and U5 are configured as voltage followers. U10 is configured as a unity gain differential amplifier, $\mathrm{C} 1$ acts as a low pass filter for noise and oscillation suppression.

A suitable electrochemical cell is not available for sampling $\mathrm{CO} 2$, thus the City Technology IRcell-CO2 is a Non-Dispersive Infra-Red (NDIR) sensor, with inbuilt temperature and pressure compensation [22]. The IRcell sensor measures the adsorption of a chopped infra-red light source through the gas sample, the wavelength of the light source determines the target gas. The IRcell sensor has a radiometric output that can be directly connected to an ADC. Figure 5.7 shows the circuity for the IRcell-CO2 sensor.

There are a number of unused op-amps in the external sensor support circuity. To prevent these from oscillating and drawing power, they are configured as a voltage follower and then the input is tied to ground. To sample the gas sensors, an ADC is needed with a voltage resolution of at least $4.2 \mathrm{mV}$ to detect a change of 2 ppm in the carbon monoxide sensor. The ADC also needs to be dual rail as the A4 sensor series can produce both negative and positive 


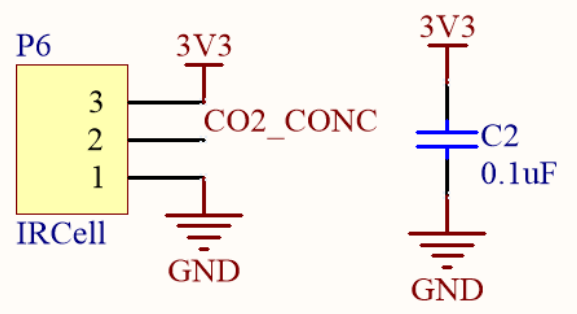

Figure 5.7: The circuity for the IRcell-CO2 sensor.

voltages. To meet these requirements, the LTC1859, 8 channel, 16 bit ADC was chosen. This ADC gives a voltage resolution of $0.15 \mathrm{mV}$ when used with a $\pm 5 \mathrm{~V}$ supply. The ADC's SPI interface means it will easily integrate with the sensor systems.

\subsubsection{Other External Sensors}

To complement the gas concentration sensors, several other aspects of the external environment can be measured to assist in rescue operations. Using barometric pressure and temperature sensors, the air velocity can be measured. Temperature sensors can be used to detect fires and when complemented with humidity sensors, they can be used to detect the presence of water.

To accurately measure the gas concentrations, the temperature and humidity of the atmosphere are used to compensate the sensors. The external air pressure is also needed to maintain the positive pressure explosion prevention system (Section 3.3 and 5.2.3.2). Table 5.3 specifies the requirements for the other atmospheric sensors and the sensors used to meet these requirements.

The external pressure sensors play a critical role in maintaining the positive pressure differential between the chassis and the external atmosphere. This pressure differential is one of vital explosive atmosphere prevention methods employed on HADES. For this reason it is paramount that these sensors return 
Table 5.3: Expected ranges and sensor selection for the other external sensors.

\begin{tabular}{|l|l|l|}
\hline Sensor & Range & Sensor chosen \\
\hline Temperature & $-5-40{ }^{\circ} \mathrm{C}$ & TMP36GRT [10] \\
\hline Humidity & $0-100 \% \mathrm{RH}$ & HIH-5030 [35] \\
\hline Pressure & $900-2000 \mathrm{mbar}$ & NPB-DPNN030PA [36] \\
\hline
\end{tabular}

an accurate reading. To ensure the readings are reliable, failure mode analysis was conducted on the pressure signal chain in order to design safeguards (Table 5.4).

Table 5.4: Failure mode analysis table for the external pressure sensing system.

\begin{tabular}{|l|l|}
\hline Failure Mode & Failure Control/ Detection Method \\
\hline Sensor Failure & Three redundant sensors, with voting algorithm \\
\hline Op-Amp failure & Voltage reference passed though Op-Amp \\
\hline ADC failure & Voltage reference for comparison \\
\hline Voltage Supply Droop & Voltage reference for comparison \\
\hline
\end{tabular}

Three sensors are used to allow the implementation of majority sensor voting (Section 8.2.3.1) [58]. To ensure the ADC is returning an accurate reading, the final pin is connected to a $1.024 \mathrm{~V}$ reference voltage, generated independently of the supply rails with a ISL21010 voltage reference IC [65]. $1.024 \mathrm{~V}$ was chosen as it approximately half of the $A_{R e f}$ voltage. If the ADC reading of the final pin changes, it indicates a fault in the op-amp or a change the $A_{\text {Ref }}$ voltage. While none of these safeguards provide full system redundancy, any fault in the pressure sensors can be identified and the operator can decide on how / if to proceed with a compromised explosive protection method.

The air pressure in underground mines can vary between 950 mbar and 1100 mbar. To sample this, an NPB-DPNN030PA pressure sensor is used. The pressure sensors used are internally configured as a Wheatstone bridge. To ensure that the full range of the sensor can be used, one side of the bridge 


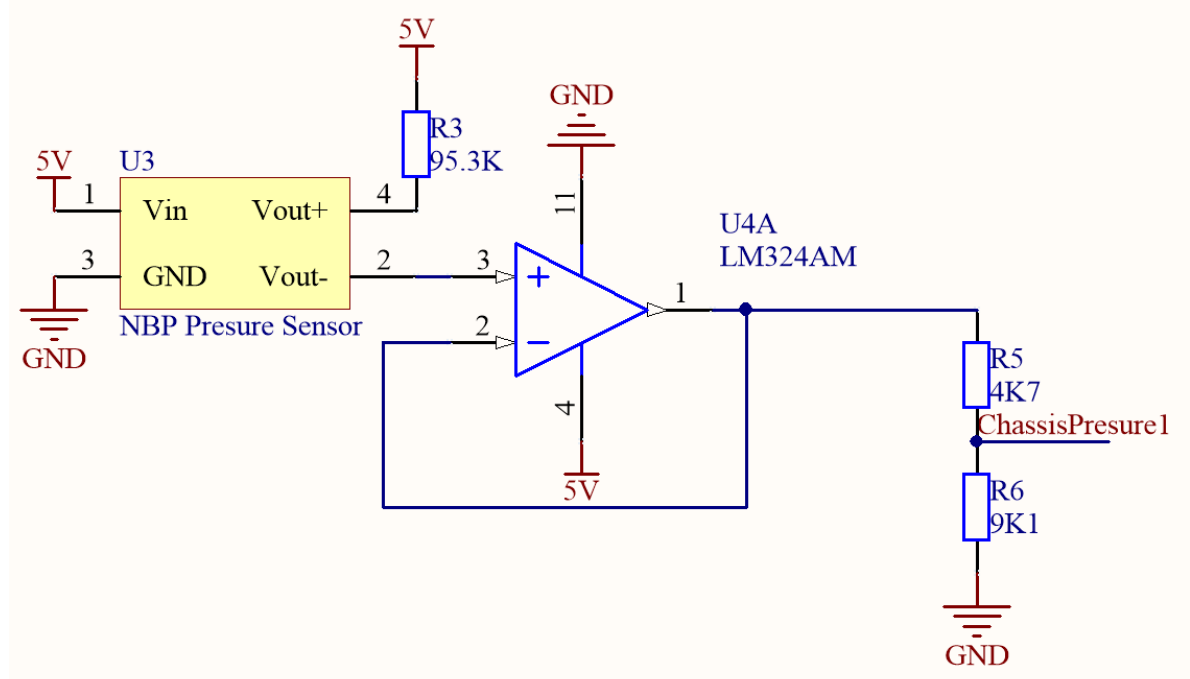

Figure 5.8: The circuity used for measuring the barometric pressure external to the chassis.

needs to be biased to keep the output voltage positive. This is achieved with R3 (Figure. 5.8), a value of $95.3 \mathrm{k} \Omega$ is used to provide a basis voltage between $35 \mathrm{mV}$ and $80 \mathrm{mV}$ depending on the ambient temperature [36].

U4A is then used in conjunction with R5 and R6 to buffer and step the 0-5 V signal down to a 0-3.3 V signal for the ADC. To prevent inaccurate ADC readings, R5 and R6 were chosen so that their combined impedance is lower than half the input impedance of the ADC. The completed pressure sensor circuit can be seen in Figure. 5.8.

The external humidity can vary from 0 to $100 \%$ relative humidity and to sample this, an HIH-5030 from Honeywell is used. This sensor produces a radiometric output voltage relative to the humidity [35]. To function correctly, the sensor needs a $100 \Omega$ bias resistor (R22, Figure. 5.9) [35]. The external temperature is sampled with two TMP36GRT sensors from Analogue Devices. These sensors produce a temperature dependent radiometric output and are accurate to $1^{\circ} \mathrm{C}$ over a rage of $-40{ }^{\circ} \mathrm{C}$ to $125^{\circ} \mathrm{C}$. 


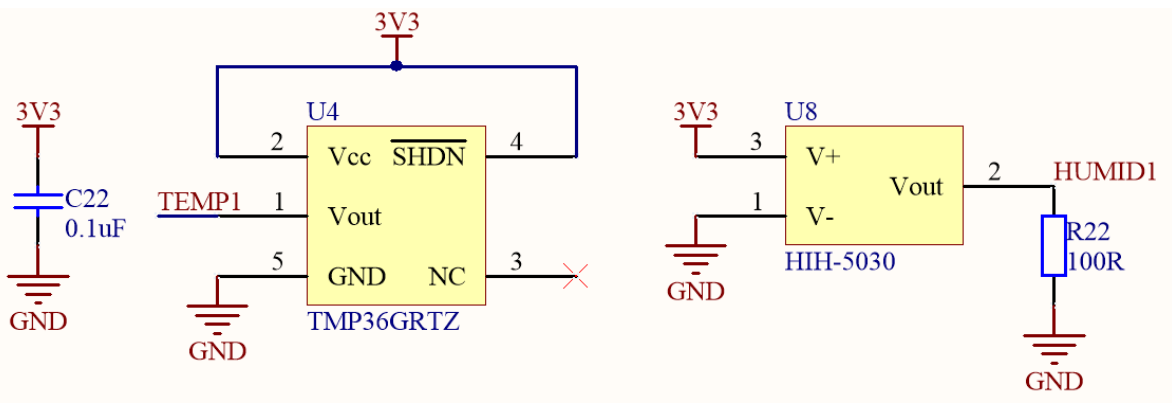

Figure 5.9: The circuity used for measuring temperature and humidity external to the chassis.

\subsubsection{External Sensor PCB}

The external sensors need to be mounted within the sensor pod and electrically connected to their support circuity via a bespoke PCB. To fit within the sensor pod (Section 3.4), the PCB should have a diameter smaller than $73 \mathrm{~mm}$ and components should not extend more than $8 \mathrm{~mm}$ above or below the PCB. As the gas sensors have a limited lifespan, the PCB should allow for easy removal of the gas sensors from the sensor pod for servicing.

The gas sensing circuity is very sensitive to noise due to the high sensitivity op-amps needed. When routing these signals, they will need to be isolated from any signal that could cause interference. The gas sensors are also temperature dependent, so care needs to be taken to avoid thermal build up around the sensors.

The gas sensor modules cannot be soldered to the PCB as the heat will damage the electrodes and as mentioned, the sensors also need to be easily replaceable due to their limited lifespan. To achieve this, $2.59 \mathrm{~mm}$ turret connectors are used, with a holding force of $0.3 \mathrm{~N}$ per connector. This is enough to retain the heaviest gas sensor (4P-50, $24 \mathrm{~g}$ ) though accelerations of up to $37 \mathrm{Nm}^{-2}$, while still being easily removable. The turret connectors can be seen in Fig. 5.10. 


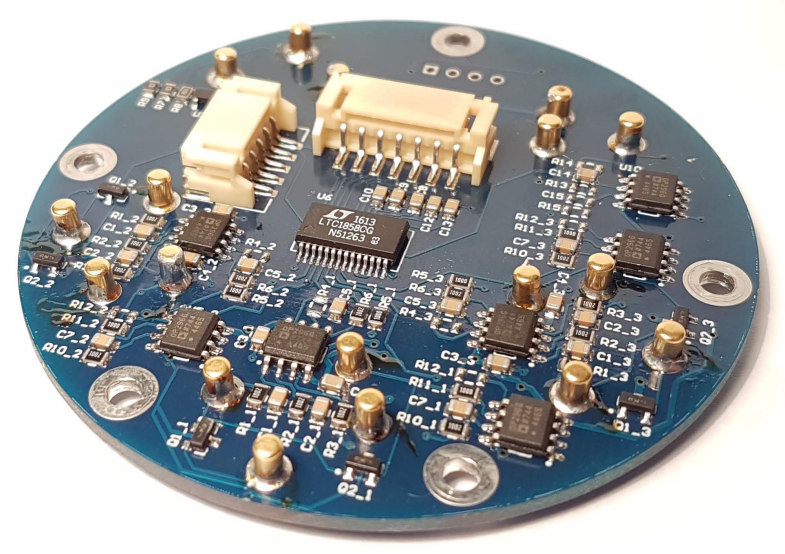

Figure 5.10: The bottom of the external sensor PCB showing the support circuity, JST and turret connectors

To allow for easy removal of the PCB from the sensor pod, the wired connections are completed with JST-PH connectors. To improve isolation, two connectors are used; one for power and one for signals. The connector wiring is designed with keyed connectors so that if the cables are accidentally swapped in installation, no components will be damaged. Figure 5.10 shows the two connectors on the bottom of the external sensor PCB.

The OP296GSZ is a $450 \mathrm{kHz}$ bandwidth op-amp [9]. It has high impedance inputs and hence is prone to oscillation and input noise. To reduce the capacitance between the op-amp inputs and ground, the ground plane is removed underneath the input pins. To minimise the chance of oscillation, the input traces of the op-amp are kept to a minimum length. Figure 5.11 shows the ground plane cut-out around the input pins on the op-amps.

To reduce the likelihood of any component heating the gas sensors, power components are placed far away from the sensors. The external temperature sensors are then placed directly next to the gas sensors so that they can be used for temperature compensation. A ground plane is placed between all the sup- 


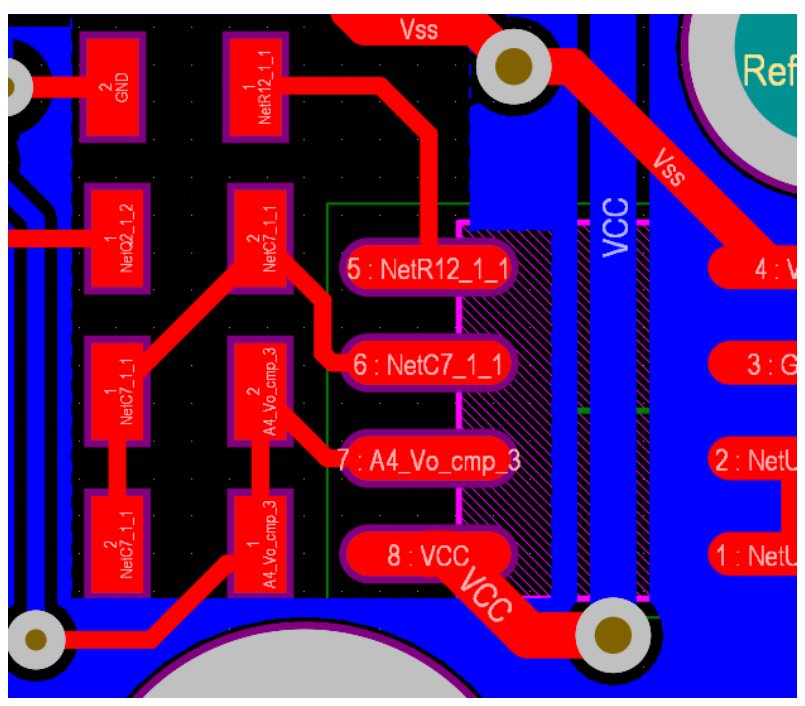

Figure 5.11: The ground plane cutout around the input pins of an OP296GSZ op-amp.

port circuity and the gas sensors to help distribute any heat.

The finished PCB is $71 \mathrm{~mm}$ in diameter and weighs a total of $75 \mathrm{~g}$ with the gas sensors installed. There are five holes for M3 screws for mounting in the sensor pod. The JST connectors allow for disconnection of the PCB from the sensor pod, with the turret connectors making replacing sensors easy. Figure 5.12 shows the finished PCB mounted to a prototype top plate of the sensor Pod.

\subsubsection{Intrinsic Safety Barrier}

As mentioned in Section 2.3 any component that is exposed to an explosive atmosphere needs to be intrinsically safe. An intrinsically safe component cannot generate or store greater than $1.5 \mathrm{~V}, 100 \mathrm{~mA}$ or $25 \mathrm{~mW}$ as defined in the A/NZ60079.11 standard [11]. Under normal operating conditions, none of the external sensors will breach these conditions. However, under fault condi- 


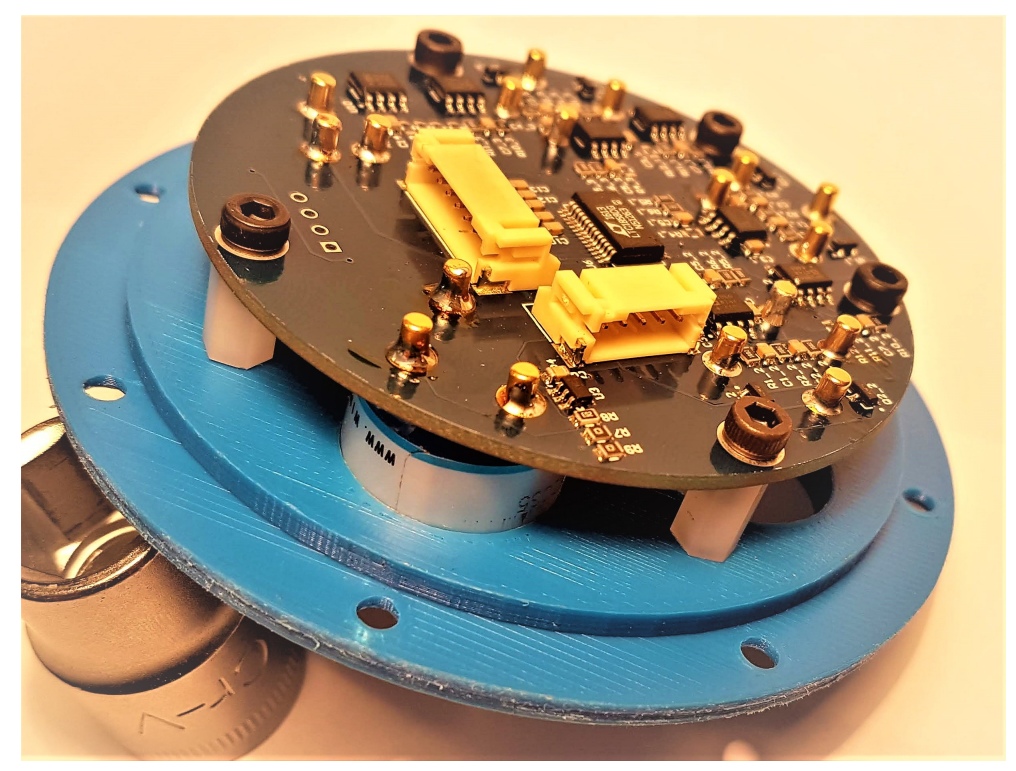

Figure 5.12: The external sensor PCB mounted to a prototype top plate of the external sensor pod.

tions, the sensors could experience large current or voltage spikes. To prevent these spikes from occurring, an intrinsic safety barrier is used. The intrinsic safety barrier is located inside the purged and pressurised chassis, so the energy spikes can be dissipated safely.

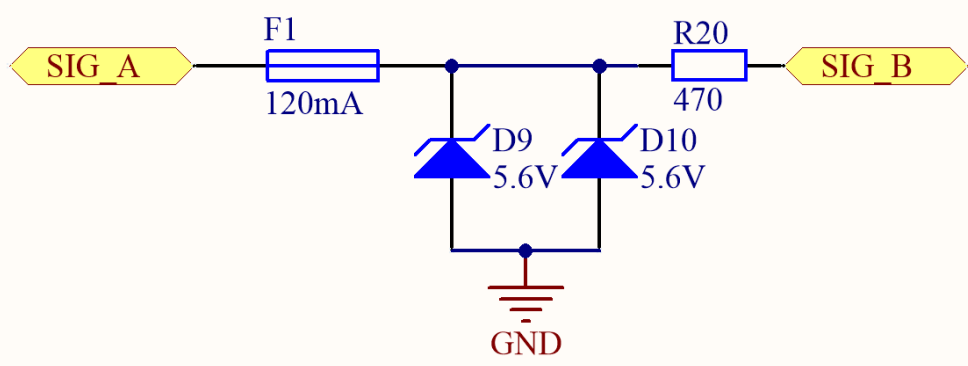

Figure 5.13: An example of an ia intrinsic saftey barrier

The New Zealand standard defines three levels of protection for an intrinsic safety barrier ( $\mathrm{ia}, \mathrm{ib}$ and ic). The protection level is defined as the number of circuit faults that can occur in the barrier before hazardous conditions are reached. 
The highest level of protection (ia) allows for two faults, with ib and ic allowing for one and zero faults respectably. Figure 5.13 shows an example of an ia level intrinsic safety barrier. Two TVS diodes are used to clamp the voltage, with a fuse and a resistor used to limit the current. In this arrangement at least two protection devices would have to fail before hazardous conditions are reached. The New Zealand standard also specifies a $1.5 \mathrm{~mm}$ minimum distance between a protected and unprotected circuit in order to prevent sparks from jumping across components.

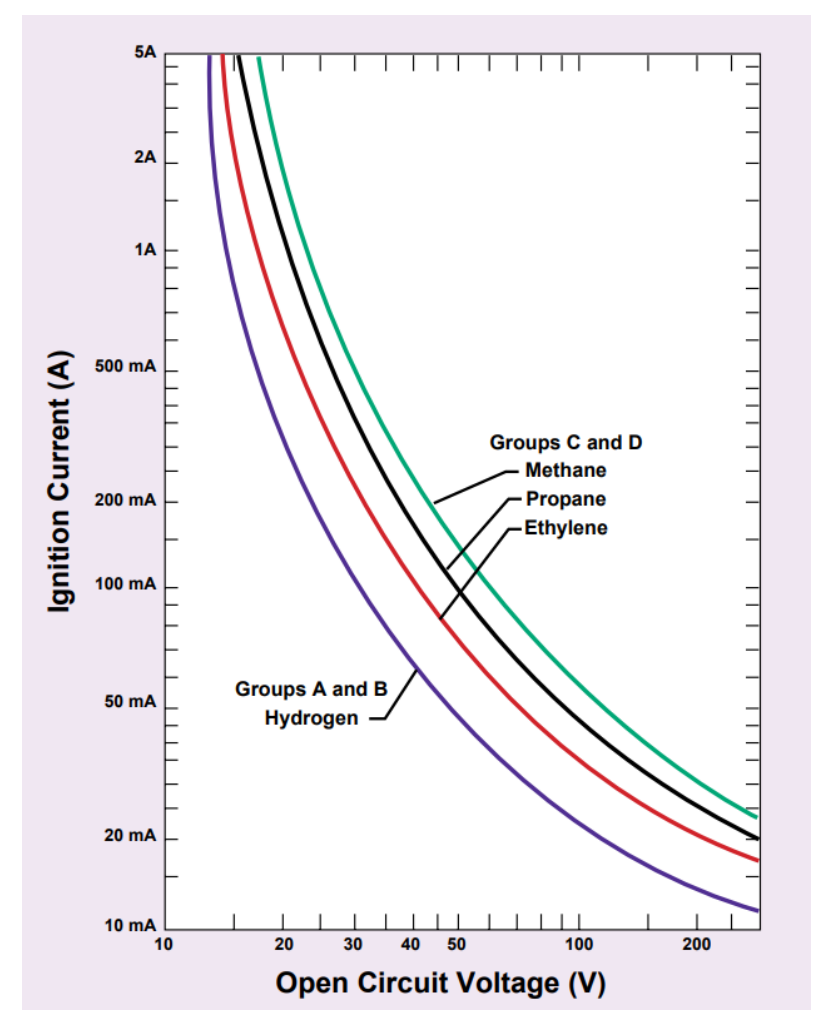

Figure 5.14: The resisitve ignition curves for common gasses found in underground coal mines [13].

Methane is the most common flammable gas in underground mines with hydrogen the most explosive. As the external sensors have no significant capacitance or inductance, the resistive ignition curve for these gasses can be used (Figure. 5.14) [13]. From this curve it can been seen that voltages below $10 \mathrm{~V}$ are 
very unlikely to cause an explosion. As all the signals for the external sensors are below $5 \mathrm{~V}$, TVS diodes with a maximum clamping voltage of $5.6 \mathrm{~V}$ are used, this falls below the $10 \mathrm{~V}$ threshold with a safety factor of 1.8. Two TVS diodes are used to provide ia level protection, each diode can sink 75 A of current before the clamp voltage climbs.

As the external sensor PCB will draw more than $100 \mathrm{~mA}$, the supply is limited to $100 \mathrm{~mA}$ with current clamps. If a current of $100 \mathrm{~mA}$ is maintained, the voltage would have to increase to $30 \mathrm{~V}$ before hazardous conditions are reached, this allows for a safety factor of 8 . A $120 \Omega$ resistor is used to limit the current to $46 \mathrm{~mA}$, thin film resistors are used as they will always fail to an open circuit [15]. To pass the current and voltage, the resistor must have a power rating of at least $0.35 \mathrm{~W}$. To achieve this, imperial 1206 package resistors are used which can dissipate $0.5 \mathrm{~W}$ of heat. The 1206 resistors also meet the circuit separation requirements of the A/NZ60079.11 standard.

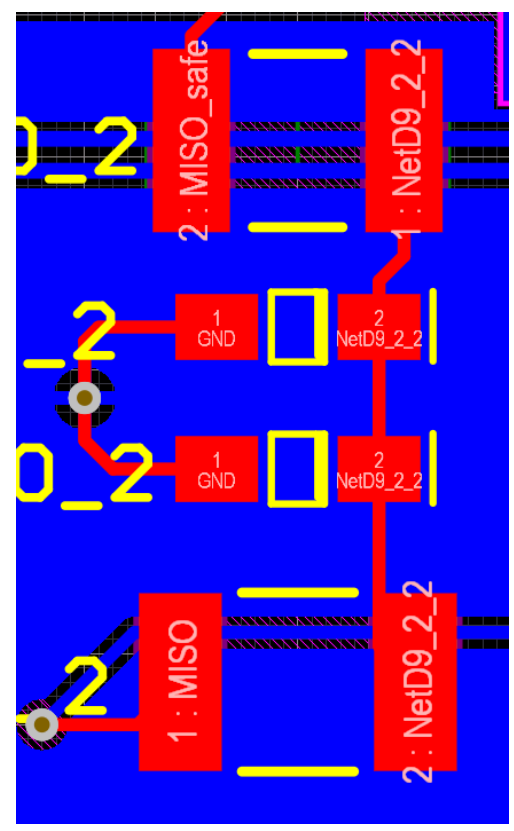

Figure 5.15: The layout of an intrinsic safety barrier used in HADES.

A PTC poly-fuse is used as the fuse element in the barrier and is chosen to 
have a continuous current of $120 \mathrm{~mA}$ with a trip current of $200 \mathrm{~mA}$. While this exceeds the design specification, the increased current will not cause hazardous conditions as the energy levels are still well below the ignition curve.

The intrinsic safety barriers are implemented on the internal sensor PCB (Section 5.2.3). The PCB layout is arranged so that the $1.5 \mathrm{~mm}$ minimum clearance is maintained. Figure 5.15 shows the PCB layout of the safety barriers. To prevent the ground potential raising during fault conditions, care is taken to ensure a low impedance ground return. Because during fault conditions the barriers can dissipate up to $0.75 \mathrm{~W}$ of power, a power plane is placed below the components to help dissipate the heat build-up.

\subsubsection{Internal Sensors}

The internal conditions of the chassis need to be monitored to ensure that all components are functioning correctly. The temperature, humidity and pressure all affect the operation of the internal electronics. The internal sensors should report motion feedback to the operator for navigation and mapping. The internal sensors will also be used for maintaining the positive pressure explosion prevention system.

The internal temperature of the chassis must remain below $50{ }^{\circ} \mathrm{C}$ so all the electronics can function correctly (Section 2.4). Accurate temperature monitoring of key electronic and mechanical components is needed so that the operator can identify and act on potential faults. There are 23 independent temperature sensors on key components throughout HADES; Table 5.5 identifies their location and purpose.

The HADES chassis is designed to meet the IP68 ingress protection standard and remain airtight during operation (Chapter 3 ). This means that in normal operation, no water should be able to enter the chassis as it could damage 
Table 5.5: The location and use of the different temperature sensors throughout the HADES chassis

\begin{tabular}{|l|l|l|}
\hline Num Sensors & Location & Use \\
\hline 6 & Batteries & Li-Po battery management \\
\hline 4 & ESCs & ECS drive transistor monitoring \\
\hline 4 & Motors & Motor/gearbox monitoring \\
\hline 2 & Power PCB & Power regulator monitoring \\
\hline 2 & Power PCB & Relay/motor current sense monitoring \\
\hline 2 & Internal sensor PCB & Internal air temperature monitoring \\
\hline 2 & IMU breakouts & IMU calibration \\
\hline 1 & Front Camera Bar & LED monitoring \\
\hline
\end{tabular}

the electronics. Therefore the sensor system needs a method for sensing water ingress.

The nitrogen gas used by the positive pressure explosion prevention system is a dry gas and so any water will be quickly absorbed [79]. By monitoring the humidity of the atmosphere inside the chassis, any water ingress can be detected. An HIH-5030 humidity sensor is used, this is the same sensor as used on the external sensors [35].

\subsubsection{Robot Pose Sensing}

To navigate and map after a disaster, the operator needs to have reliable and accurate data about the position and pose of the robot. This data must allow the operator to understand how far the robot has travelled as well as the current orientation of the chassis. The data should be in a frame of reference that the operator understands (i.e. relative to Earth's magnetic field and gravity).

The positional data can be gathered from the odometry by integrating the 
motor commutation cycles (Section 6.1). However, this method is likely to be inaccurate and will drift significantly over time. Therefore, additional position data is needed.

Inertial Measurement Units (IMU) measure angular and linear accelerations to provide motion feedback. To reference the pose to Earth's magnetic field, the IMU will need to contain a magnetometer. To maintain an accurate pose estimate, given possible robot motions, the IMU should to be able to sample linear accelerations up to $100 \mathrm{~ms}^{-2}$ and rotational accelerations up to $500 \mathrm{degs}^{-2}$ at a sample rate of greater than $500 \mathrm{~Hz}$.

The MPU-9250 is a nine-axis accelerometer, gyroscope and magnetometer, capable of measuring linear accelerations up to $160 \mathrm{~ms}^{-2}$ and rotational accelerations up to $2000 \mathrm{degs}^{-2}$. To measure the pose of the robot, two MPU-9250 IMUs are used, one in the main body and one in the rear arm.

When all nine axes are combined with a sensor fusion algorithm, the pitch, yaw and roll of the robot can be calculated. The sensor fusion algorithm can also integrate the accelerations to provide an estimation of the distance travelled. The sensor fusion algorithms are discussed in Section 8.2.2.

The MPU-9250 can communicate with I2C or SPI. To meet the sensor system design requirements (Section 5.1) SPI is used which allows a maximum sample rate of $10 \mathrm{kHz}$. Figure 5.16 shows the schematic for the IMU. To prevent high currents from the motors interfering with the magnetometer, care is taken to place the IMUs as far as possible from the motors, ESCs and cables.

\subsubsection{Positive Pressure Explosion Protection System}

For the system to meet the A/NZS60079.2 standard, the chassis must maintain a higher pressure than atmosphere by $50 \mathrm{~Pa}$ and the atmosphere inside the chassis must be less than $1 \%$ oxygen [12]. To achieve this, the chassis is filled 


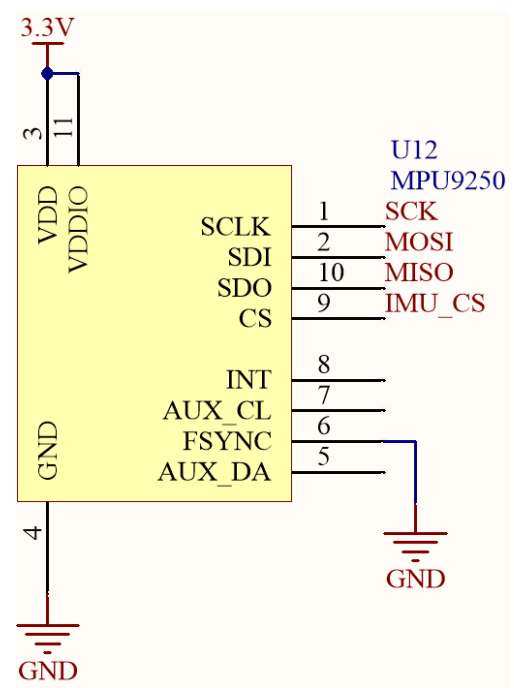

Figure 5.16: The schematic implementation of the MPU9250 IMU.

with inert nitrogen gas from an on-board $213 \mathrm{~cm}^{3}, 3000$ PSI tank. The release of nitrogen from the tank is controlled with a solenoid valve. Two extra solenoid valves control the external fill and purge ports. The full implementation of the positive pressure gas system is discussed in Section 3.3.

To maintain the pressure differential, the internal chassis pressure must first be known. Like the external pressure sensors, the internal pressure sensors are critical for maintaining the positive pressure explosion prevention system. For this reason, the same failure mode analysis is applied to the internal sensors, resulting in an identical pressure measurement system to the external sensors. Using the same sensing setup simplifies the control loop as the two pressure measurements can be directly compared without sensor compensation. Care will need to be taken in the control system to compensate for the $3 \mathrm{~s}$ response time of sensors, as the gas system response is much faster than the pressure sensors.

The pressure of the nitrogen tank will indicate how much gas is remaining. To assess this, a M5231-03KPG 0 - 3000 PSI pressure transducer is used [72]. The 


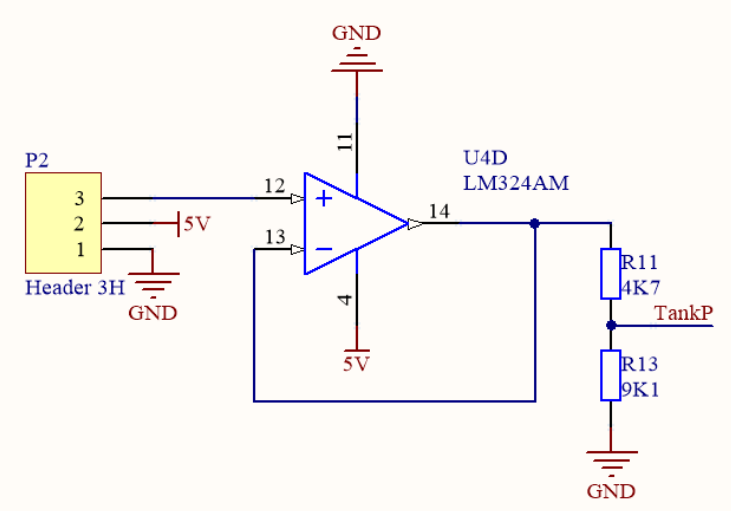

Figure 5.17: The circuit for converting the $5 \mathrm{~V}$ signal from the tank pressure sensor to a $3.3 \mathrm{~V}$ signal for the ADC.

sensor has a $0.5-5 \mathrm{~V}$ radiometric output, this is reduced to a $0.3-3.3 \mathrm{~V}$ signal for the ADC, using a buffer and voltage divider (Figure. 5.17). A sensor fault condition can be detected if the voltage falls bellow $0.3 \mathrm{~V}$.

To measure the internal oxygen concentration, the same ME2-O2 sensor is used as the external sensors. However, the load resistors of the support circuitry are changed from $100 \Omega$ to $470 \Omega$, to increase the sensor's response linearity between 0 - $5 \%$ [34].

The solenoids are controlled with a low-side N-MOS transistor and the flyback voltage is suppressed by D3 (Figure. 5.18). To smooth the in-rush current to the solenoid, a $100 \mu \mathrm{F}$ capacitor is placed near the switching transistor. To allow for servicing and testing, buttons are added to control each solenoid. To assist in tuning the control system, an indicator LED is added to the gate of each transistor. 


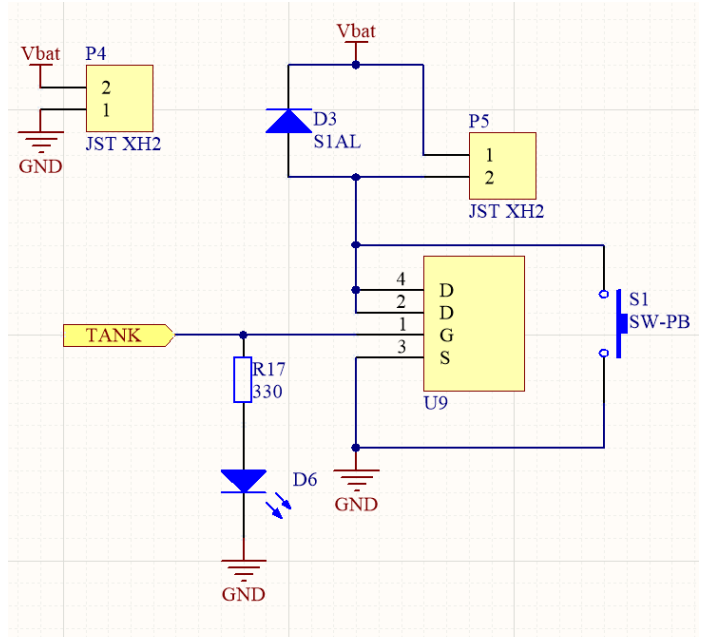

Figure 5.18: The solenoid driving circuitry for the positive pressure control system.

\subsubsection{Internal Sensor PCB}

The internal sensors and positive pressure control electronics all need to be mounted and electrically connected. A dedicated PCB is used to implement this. To minimise the length of the wires needed to link the external and internal sensor PCBs, the internal sensor PCB should mount onto the bottom of the sensor pod. The internal sensor PCB needs to allow for the wiring to exit the centre of the sensor pod and connect to the PCB.

To allow the internal sensor PCB to mount to the bottom of the sensor pod, it is circular in shape and has a diameter of $86 \mathrm{~mm}$. There is a slot in the centre of the PCB to allow for the wiring pass through, and two JST-PH connectors are located adjacent to the slot. To prevent the intrinsically safe signals from connecting to the un-protected signals, JST-XH connectors are used as they will not mate with the JST-PH connectors. Figure 5.19 shows the internal sensor PCB with connectors and pass through slot. 


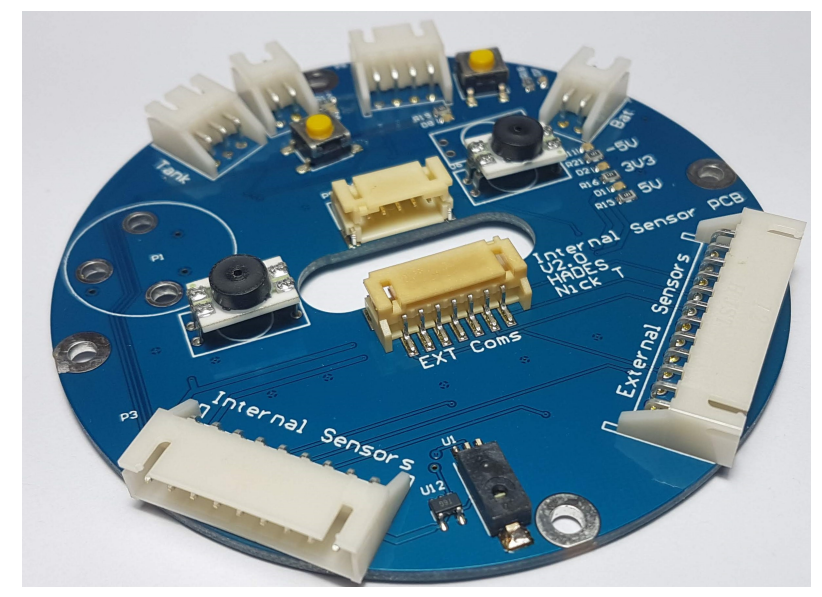

Figure 5.19: The internal sensing PCB, without the oxygen sensor.

\subsubsection{Sensor Systems Summary}

The operator needs to ascertain the gas levels of an underground mine to ensure that it is safe for human entry. The external sensors will be in contact with a potentially explosive atmosphere and therefore will need to meet the A/NZ60079.11 intrinsic safety standard. The operator also needs to be aware of the conditions inside the chassis to ensure the electronics don't overheat and the A/NZ60079.29 positive pressure standard is met. The external gas conditions are measured with five electrochemical cells and one NDIR cell. Together they sample seven of the most common gasses in underground mines, in the ranges that affect explosivity and human health. The gas sensors all have bespoke analog front ends to ensure the gas readings are accurate. The external humidity, barometric pressure and temperature are also measured to provide additional data about the mine.

The internal chassis sensors measure the temperature, air pressure, oxygen concentration and humidity of the internal atmosphere. The internal sensors enable the positive pressure system to maintain a pressure differential of $50 \mathrm{~Pa}$ between internal and external atmospheres. The internal sensor system also 
uses two MPU-9250 inertial measurement units to measure the pose and position of the robot. The internal and external sensors are mounted on two bespoke PCBs that mount to the sensor pod.

\subsection{Camera Systems}

\subsubsection{Requirements}

HADES will be working in dark conditions, often navigating through complex terrain. The camera systems on HADES will need to supply the operator with images so they can drive the robot and make informed decisions.

The operator needs to be able to see far enough in front of the robot to react to oncoming obstacles. The average human can react to a video feed in less than $1 \mathrm{~s}$ [55]. When combined with the worst case communication system latency (500 ms one way), the worst case total reaction time is $2 \mathrm{~s} \mathrm{[29].} \mathrm{Therefore,} \mathrm{when}$ the robot is travelling at cruising speed $\left(1.4 \mathrm{~ms}^{-1}\right)$ the operator needs to be able to see at least $2.8 \mathrm{~m}$ in front of the chassis.

Depth cameras have been proven to help operators better navigate obstacles and to reduce the key-hole effect [21]. To assist rescue efforts, the depth camera data can also be used in conjunction with pose and odometry sensor data to produce a 3D map of the mine.

Thermal cameras have been used successfully in search and rescue operations for locating survivors [68]. A thermal camera on HADES will have two purposes, to search for survivors and identify fire/heat hazards. To achieve this, the thermal camera needs to have a precision of at least $5{ }^{\circ} \mathrm{C}$ and an operating range from $0{ }^{\circ} \mathrm{C}$ to $200{ }^{\circ} \mathrm{C}$.

As well as looking forward, the operator needs to be able to see around the 
robot. To aid the operator to navigate complex terrains, the side looking cameras should be able to see the ground contact points of both Whegs (Section 4.1). The side looking cameras will also be used when deploying the communication nodes.

Underground mines are often in complete darkness after a disaster as lighting is damaged or switched off for safety. To gather any visual information a lighting system is required. The lighting system should allow all the cameras on HADES to render images at-least $3 \mathrm{~m}$ from the robot. As the communication link only has enough bandwidth for one video stream, all the video data should be stored on the NUC for retrieval later.

\subsubsection{Implementation}

\subsubsection{Depth Camera}

There are two common depth cameras used in robotic development; the Intel Realsense and Microsoft Kinect. Both these camera systems include integrated depth, visual and IR camera systems. At the time of development, there are two Realsense camera modules available, the R200 and the SR300. Table 5.6 shows a feature comparison between all three cameras.

Table 5.6: A comparison between the Microsoft Kinect, SR300 and R200 depth cameras.

\begin{tabular}{|l|l|l|l|l|l|}
\hline Camera & Resolution & Depth Range & Depth Resolution & Current & Volume \\
\hline Kinect & $640^{*} 480$ & $0.5-4.5 \mathrm{~m}$ & $640^{*} 480$ & $2.8 \mathrm{~A}$ & $788 \mathrm{~cm}^{3}$ \\
\hline SR300 & $1920^{*} 1080$ & $0.2-1.2 \mathrm{~m}$ & $1920^{*} 1080$ & $0.6 \mathrm{~A}$ & $15.8 \mathrm{~cm}^{3}$ \\
\hline R200 & $1920^{*} 1080$ & $0.5-5 \mathrm{~m}$ & $640^{*} 480$ & $0.5 \mathrm{~A}$ & $6.8 \mathrm{~cm}^{3}$ \\
\hline
\end{tabular}

Although the Kinect does have better depth sensing capabilities compared to Realsense cameras, its large size and high current draw discount it for use in 
HADES. The Realsense R200 camera has comparable depth sensing capabilities to the Kinect but with a higher resolution visual camera and a much smaller form factor. The SR300 Realsense has a smaller depth sensing range, but with a higher resolution camera, with a lower ISO capability. The lower the ISO of a camera, the better it will perform in low light conditions. Both the R200 and the SR300 were purchased to investigate the trade-off between depth data and low light performance.

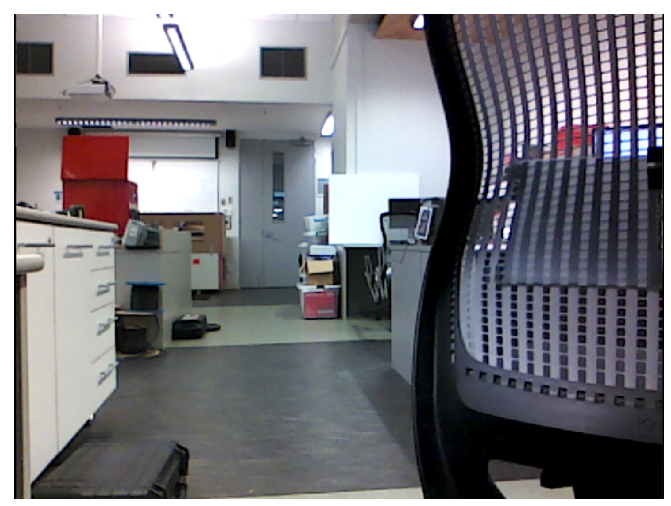

(a)

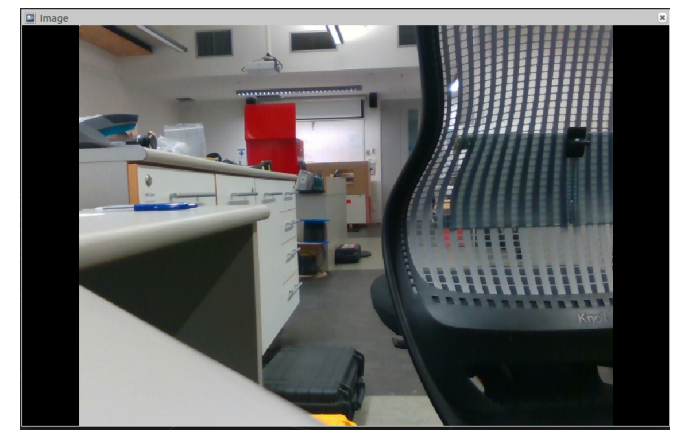

(b)

Figure 5.20: Images of a test scene with a R200 (left) and SR300 (right) Realsense Camera.

Figure 5.20 shows two test images taken of the same scene with the SR300 and R200 cameras; light levels at the centre of the image were $200 \mathrm{Lux}$ at $1.5 \mathrm{~m}$. The R200 image has much more graininess and appears to be lower quality. The SR300 image is clearer and brighter and it is easier to identify features. Figure 5.21 shows the two depth images from each camera. As the depth imaging system on the Realsense cameras is dependent on the lighting conditions, the same scene is used as in Figure 5.20.

As the visual images are going to be used more than the depth images for robot navigation, the SR300 camera was chosen. This camera is mounted on an aluminium strut (Section 3.1.4) across the front windscreen. This strut also supports the thermal camera and the LED lighting. Figure 5.22 shows the forward- 


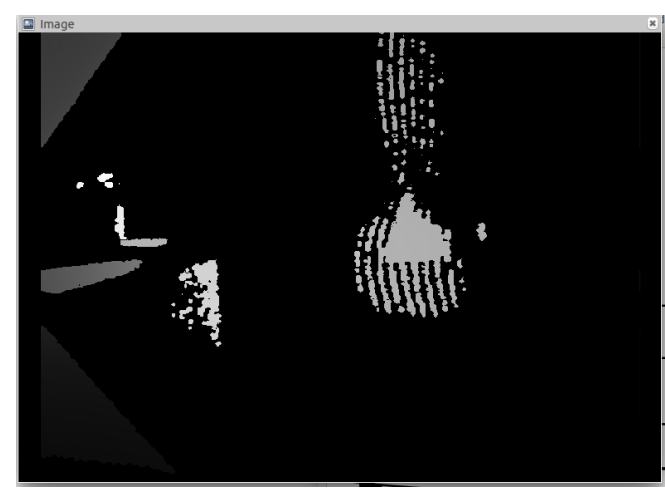

(a)

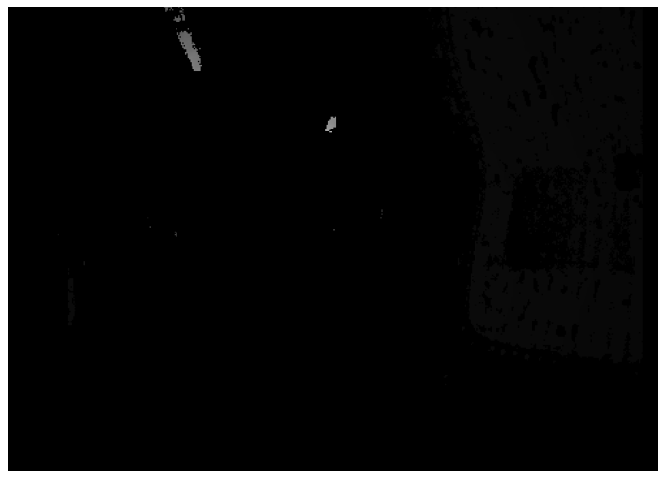

(b)

Figure 5.21: Depth images of a test scene with a R200 (left) and SR300 (right) Realsense Camera.

looking cameras mounted inside the HADES chassis.

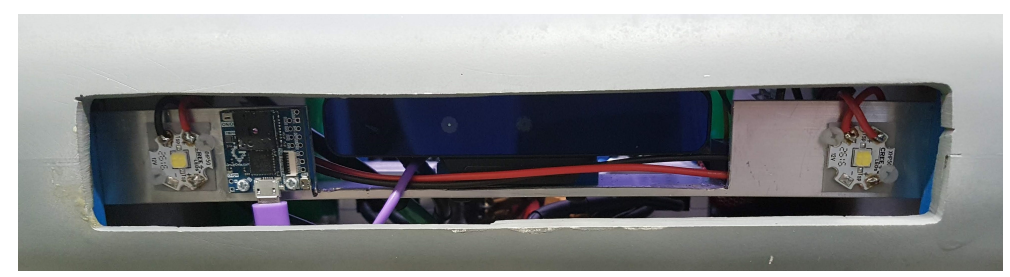

Figure 5.22: The front cameras and LEDs mounted inside HADES.

\subsubsection{Thermal Camera}

The Pure Thermal 1 is a USB thermal camera based on the FLIR Lepton thermal imaging system. The FLIR lepton module has a resolution of $60 \times 80$ pixels and thermal resolution of $50 \mathrm{~m}^{\circ} \mathrm{C}$. During initial testing in a dark hallway, it was found that a human form could be identified at $40 \mathrm{~m}$ in complete darkness (Figure 5.23).

The thermal camera is mounted to the same aluminium strut as the other front looking cameras. As this strut acts as a heat sink for the lighting (Sec- 


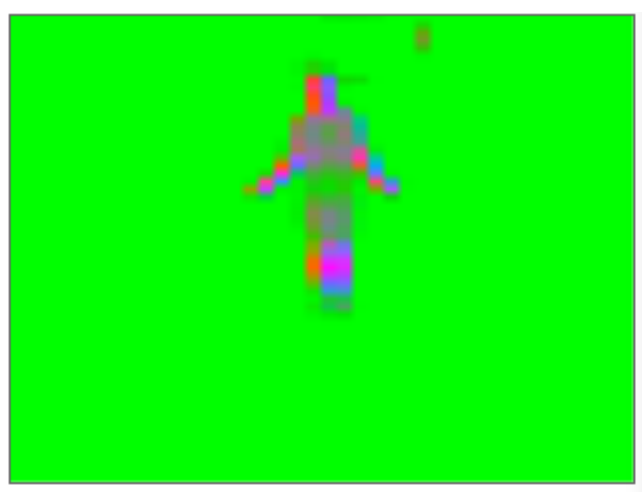

(a) A human at $5 \mathrm{~m}$

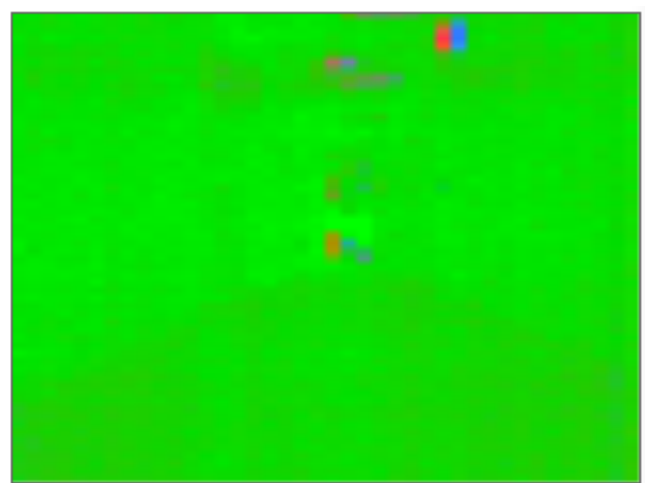

(b) A human at $40 \mathrm{~m}$

Figure 5.23: Thermal images of a human taken with a Pure Thermal 1 at 5 and $40 \mathrm{~m}$ in a dark hallway.

tion 5.3.2.4), the thermal camera is isolated with an ABS pad to prevent thermal interference.

\subsubsection{Side and Rear Looking Cameras}

To allow the side looking cameras to see the ground contact points and the communication node dispensers, they will need a viewing angle of $160^{\circ}$. To achieve this viewing angle, a fisheye lens is needed. Figure 5.24 shows an image from the fisheye camera mounted on the side of the chassis. Fisheye cameras have distorted edges and so to compensate for this, correction algorithms are used (discussed in Section 7.1.2).

The rear looking camera is a Microsoft LifeCam as it is low cost and has a relatively good low light performance. To reduce the space needed for the camera, the casing is removed. Figure 5.25 shows the camera with the casing removed. 


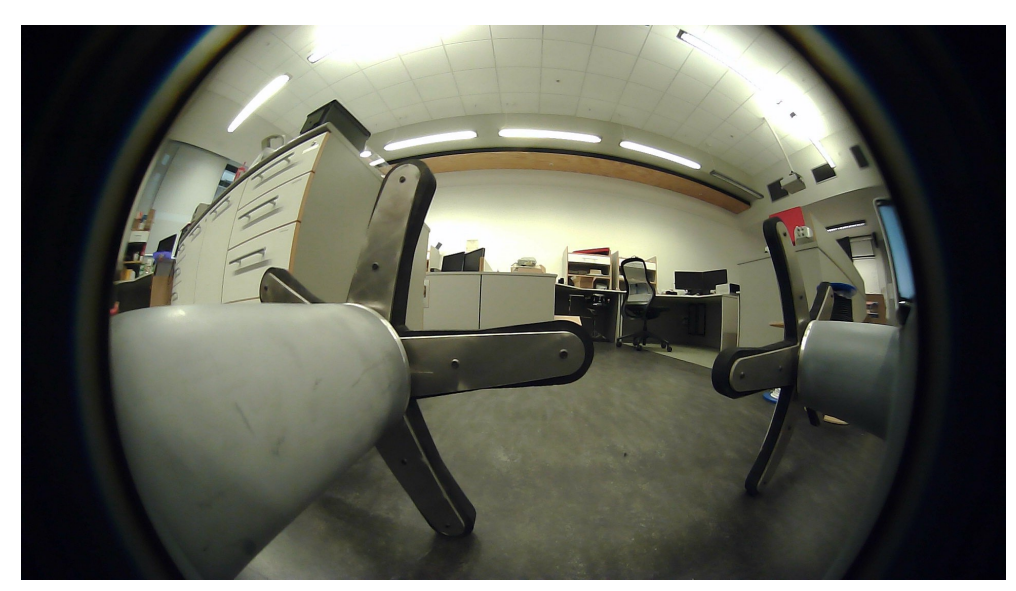

Figure 5.24: An image from the side looking fish eye camera.

\subsubsection{Lighting}

Underground mines typically are in complete darkness post disaster and so for the visual cameras to be used for navigation, lighting systems are needed. As well as providing sufficient illumination, the lighting system should be low power, low heat output and with the option of being operator controlled. LEDs have better efficiency and lower heat output compared to halogen, incandescent or fluorescent light sources and therefore are the obvious choice. However, care needs to be taken when selecting LEDs to ensure that the spectrum doesn't interfere with the Realsense depth camera and the thermal camera.

The intensity and colour temperature of the lighting is also important as this determines the maximum range and dust penetration of the lighting. Lower temperature lighting has been observed to penetrate through dust and water, while higher colour temperature provides better colour accuracy and illumination in complete darkness. To balance dust penetration and colour accuracy, a colour temperature between $4800 \mathrm{~K}$ and $5500 \mathrm{~K}$ is recommended [56]. During initial trials with the SR300 camera it was found that with $900 \mathrm{~lm}$ at a colour temperture of $5000 \mathrm{~K}$ a human form could be identified at $20 \mathrm{~m}$ at night. 


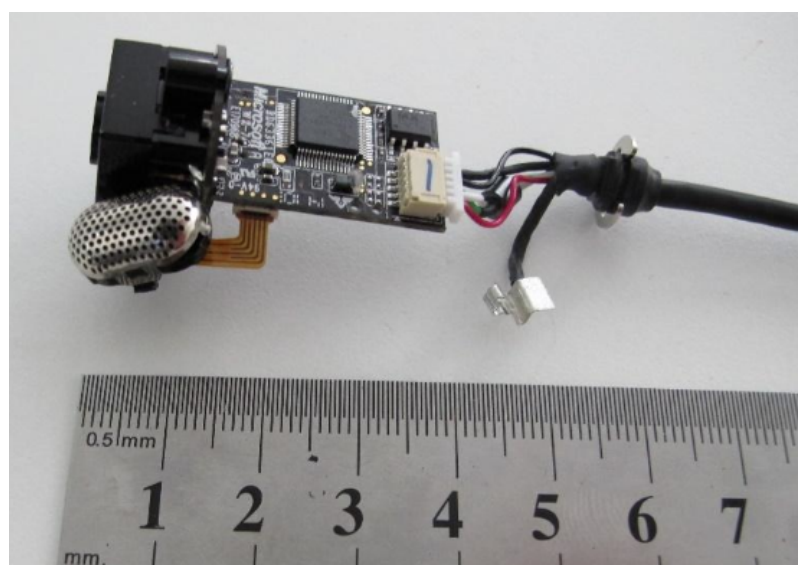

Figure 5.25: The Microsoft LifeCam with casing removed

To meet this illumination requirement, XHP50A LEDs are used. These have a total output of $1120 \mathrm{~lm}$ each and a colour temperature of $5000 \mathrm{~K}$. Five LEDs are mounted in HADES, two looking forward and one for each of the other cameras. The LEDs are driven with Meanwell LDD-700H constant current LED drivers. Each LED cluster is individually controllable via PWM. To prevent image flickering, the LDD modules have a switching frequency of $40 \mathrm{kHz}$. The LDD modules are mounted on the auxiliary system's PCB, Figure. 5.26 shows the schematic for the LDD modules.

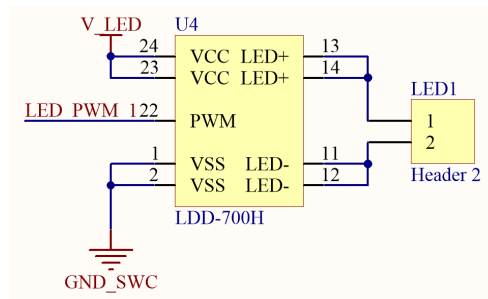

Figure 5.26: The schematic implementation of the LDD-700 constant current drivers.

Each LED can dissipate 7.2 W of heat and if they are left uncooled, the operational life of the LEDs will be significantly reduced. To mitigate this, the camera mounting strut shown in Figure 5.22 is used to heat sink the front LEDs. Ini- 
tial testing showed that after 2 hours of operation the temperature of this strut settled at $34{ }^{\circ} \mathrm{C}$, this is below the $50{ }^{\circ} \mathrm{C}$ maximum stated in the requirements section (Section 2.4).

The side and rear looking LEDs are automatically switched off when the camera isn't active by the camera management software (Section 7.1.2), smaller heat sinks can be used. To cool the side LEDs, Fin Star heat sinks are used and are mounted with 3D printed brackets. The rear LED is cooled by sinking the heat into the aluminium rear panel.

\subsubsection{Camera Systems Summary}

The operator needs a view around the robot to successfully navigate and ascertain the condition of the mine. As the mine is expected to be in complete darkness the camera system needs to be supplemented with a lighting system to increase the light levels of the images. To view the surroundings of the robot a five camera system is used. The camera system allows the operator visual images around the chassis, with depth and thermal images looking forward to assist with navigation and locating survivors. A low power LED lighting system of correct colour temperature to improve colour rendition and dust penetration, provides illumination around the robot. 


\section{Chapter 6}

\section{Electronics - Motor Drive and Power Management}

\subsection{Motor Drive and Control}

Brushless DC motors require external commutation for operation which is usually implemented with an Electronic Speed Controller (ESC). An ESC switches the electrical currents in the motor coils to create mechanical movement. The speed of switching and amount of current passed determines the output speed and torque of the motor. When a brushless motor is used with a reduction gearbox, the output torque $(\tau)$ and speed $(\omega)$ for a supplied voltage $(V)$ and current $(I)$ is given by,

$$
\tau=R_{i} K_{t} I
$$

and,

$$
\omega=\frac{K_{v} V}{R_{i}}
$$

where $K_{v}$ is the motor velocity constant, $K_{t}$ is the motor torque constant and $R_{i}$ is the gearbox reduction ratio. The drive systems in HADES all use a planetary 
gearbox with a reduction ratio of 64:1 (Section 4.2).

It is generally accepted that an ESC should be able to supply at least $150 \%$ of the maximum currents required by its motor [18, 32]. The Hobbystar 56112 motors are capable of drawing 40 A continuously with peak currents of $180 \mathrm{~A}$. Thus the ESC should be able to supply at least $60 \mathrm{~A}$ of continuous current with 270 A peaks.

To prevent HADES from rolling on steep slopes, the ESC should be able to brake the motor. To hold the mass of HADES steady with one Wheg in traction, the motor/gearbox system will need to provide $72 \mathrm{Nm}$ of holding torque. Using the 64:1 gearboxes and the Hobbystar 56112 motors $\left(K_{t}=0.075 \mathrm{NmA}^{-1}\right)$ the ESC will need to sustain at least $15 \mathrm{~A}$ of brake current (Eq. 6.1).

Some modern ESCs can be configured to match the connected motor; this improves the linearity of the torque response especially at low speeds [45]. Some configuration utilities also allow the ESC to limit the maximum current, speed and temperature of the motors protecting the motor and the ESC. For these reasons, an ESC that can be configured is safer and can deliver more torque in low-speed operation, thus being suited for use in HADES.

There are two common sensor-less commutation methods for driving brushless DC motors, Field Oriented Control (FOC) and trapezoidal commutation. In trapezoidal commutation mode, the ESC will switch the currents in the motor coils, while in FOC mode the current is modulated to form a sine wave. Figure 6.1 shows the different current waveforms between trapezoidal and FOC commutation.

FOC commutation is generally more efficient and can provide torque at all rotor positions, unlike trapezoidal commutation [45, 42]. However, FOC commutation needs to be accurately tuned to each motor and has a lower top speed than trapezoidal commutation. As the Hobbystar 56112 motors used will be running at $20 \%$ of their maximum speed, FOC control is preferred (Section 4.2). 


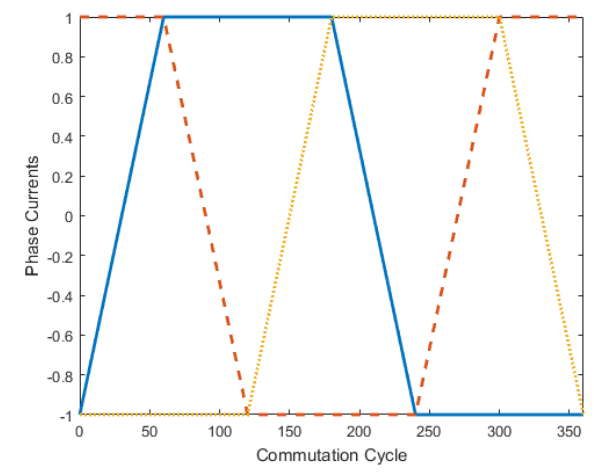

(a) The motor coil current waveform for trapezoidal commutation

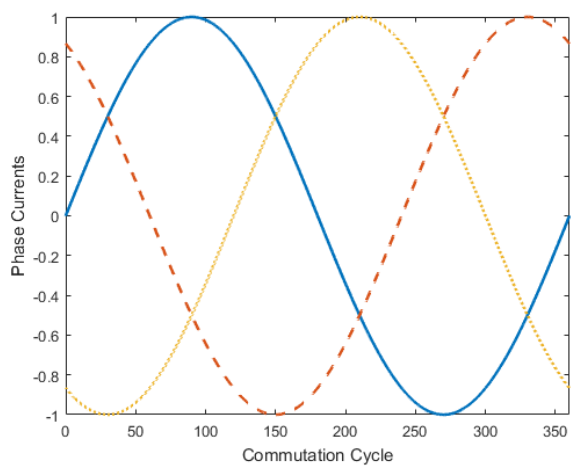

(b) The motor coil current waveform for FOC commutation

Figure 6.1: A comparison of trapezoidal and FOC commutation methods

Table 6.1 shows some possible options for ESC selection.

Table 6.1: A comparison of the different ESCs available for the HobbyStar 56112 BLDC motors.

\begin{tabular}{|l|l|l|l|l|l|l|}
\hline Name & I Cont & I Peak & V Max & Config & Interfaces & FOC \\
\hline EXRUN Max5 & $200 \mathrm{~A}$ & $1300 \mathrm{~A}$ & $30 \mathrm{~V}$ & No & PPM & No \\
\hline ToroBeast & $200 \mathrm{~A}$ & $800 \mathrm{~A}$ & $30 \mathrm{~V}$ & No & PPM, PWM & No \\
\hline FOCBOX & $70 \mathrm{~A}$ & $180 \mathrm{~A}$ & $60 \mathrm{~V}$ & Yes & $\begin{array}{l}\text { PPM, UART, } \\
\text { I2C, CAN, USB }\end{array}$ & Yes \\
\hline VESC & $70 \mathrm{~A}$ & $300 \mathrm{~A}$ & $80 \mathrm{~V}$ & Yes & $\begin{array}{l}\text { PPM, UART, } \\
\text { I2C, CAN, USB, } \\
\text { BTLE }\end{array}$ & Yes \\
\hline
\end{tabular}

Two ESCs that support FOC and are capable of driving the current required for the Hobbystar 56112 motors are the Vedder ESC (VESC) and the FOCBOX. Both these ESCs are developed from reference designs developed by Benjamin Vedder for use in electric skateboards. Two hobby ESCs are included in Table 6.1 for comparison. While these have significantly larger current driving 
capabilities, they lack the communication and control functionality needed for use in HADES. At the time of ESC selection the FOCBOX was still in beta testing, and therefore the VESC was chosen.

The VESC is an open source ESC, allowing for customisation of the hardware and the software. The VESC is based around the DRV8302 motor controller and an STM32 ARM F4 processor and can operate with or without rotary encoder feedback. When operating in sensor-less mode, the back EMF from the motor coils is used as position feedback for the commutation calculation. The DRV8302 also allows the VESC to use the motor coils as a buck converter, reducing the supply voltage and increasing the current; improving the linearity of the torque/speed curve and decreasing battery current at low speeds.

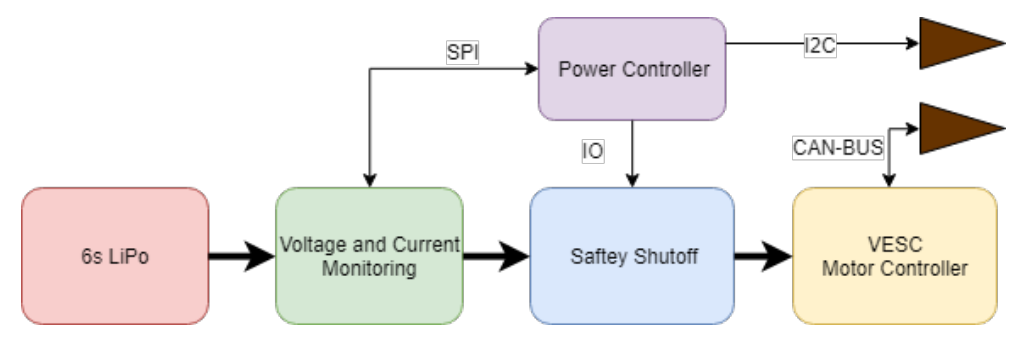

Figure 6.2: The power and control infrastructure for the connection of one of the VESCs.

Four VESCs are used in HADES, one for each of the drive motors. The VESCs are connected to a six-cell Lithium Polymer battery through a Battery Management System (BMS) (Section 6.2.2). The VESCs support control data over six different interfaces (Table 6.1), as four VESCs are used, a bussing control standard is needed to minimise connections to the main microcontroller.

I2C, SPI and CAN-BUS were investigated, CAN-BUS was chosen as it has the highest EMF rejection properties of the supported communication standards. CAN-BUS is a parallel bussing standard that requires two $120 \Omega$ termination resistors at endpoints of the network to maintain the correct network impedance. As all the VESCs are supplied with termination resistors, three of 
the VESCs were modified by removing their termination resistor. To interface between the main microcontroller and the CAN-BUS, a SN65232 transceiver is used. Figure 6.2 shows the wiring diagram for the connection of pne of the VESCs.

The firmware on the VESC can be configured with the BLDC Tool; a software interface for configuring the VESCs over the USB link. The firmware configuration is used to tune the controller to match the motor parameters and set limits for the currents, voltages and temperatures of the drive system.

The detection of the motor parameters is completed automatically by the VESCs during the setup phase of the VESC. During this time the motor is run at different speeds to detect the resistance, inductance, flux-linkage and other motor properties. Once these properties have been detected, the VESC atomically generates control system constants that will best suit the connected motor. The control system can be tuned to limit the maximum speed, current and temperature of the motor and ESC. Table 6.2 shows the limits used in HADES.

Table 6.2: The operational limits for the properties of the VESC that trigger a shutdown or soft back-off state.

\begin{tabular}{|l|l|l|}
\hline Property & Back-off & Shutdown \\
\hline Motor Current & $280 \mathrm{~A}$ & $350 \mathrm{~A}$ \\
\hline Motor Temperature & $50{ }^{\circ} \mathrm{C}$ & $70{ }^{\circ} \mathrm{C}$ \\
\hline Motor ERPM & 25000 & 30000 \\
\hline Battery Current & $150 \mathrm{~A}$ & $200 \mathrm{~A}$ \\
\hline Battery Brake Current & $20 \mathrm{~A}$ & $50 \mathrm{~A}$ \\
\hline Battery Voltage Lower & $21 \mathrm{~V}$ & $19 \mathrm{~V}$ \\
\hline Battery Voltage Upper & $25.5 \mathrm{~V}$ & $28 \mathrm{~V}$ \\
\hline ESC Temperature & $50{ }^{\circ} \mathrm{C}$ & $70{ }^{\circ} \mathrm{C}$ \\
\hline
\end{tabular}

The VESC can also limit the supply and regeneration currents for the connected battery, as well as the maximum and minimum battery voltages (Ta- 
ble 6.2). The values for these limits are set above the thresholds used by the battery management system discussed in Section 6.2.2. This means the VESC will detect, and act on any dangerous conditions that the battery management system fails to detect.

Table 6.2 lists two values for each condition; one is the shutdown state and one the back-off state. If the shutdown state is reached, the VESC immediately shuts the motor down and enters a low power state. When the back-off state is reached, the VESC reduces the output power of the drive system in order to bring the system back into a safe operating state. The main microcontroller can also control the soft back-off. This means that if one VESC is operating at reduced power, the other VESCs can also be commanded to reduce power to compensate. The implementation of the control software on the main microcontroller is covered in Chapter 8.

\subsubsection{Summary}

An ESC is needed to drive the Hobbystar 56112 brushless motors used in the HADES locomotion system. The ESC needs to be able to supply 60 A of continuous current with peaks of up to $270 \mathrm{~A}$. To meet the ESC requirements, the Vedder ESC (VESC) was chosen. The VESC allows the operator to control each of the drive motors independently, while minimising the control load on the main microcontroller. The VESCs are configured so that they limit the currents, voltages and temperatures of the connected motor and battery. If the limits are exceeded. the VESC will implement a soft back-off strategy, where the output power of the VESC is reduced. If one VESC reduces power, the other VESCs in the system will reduce power to compensate. 


\subsection{Power Supply and Management}

HADES needs to operate for at least 4 hours self-sufficiently to cover the entirety of our target underground mine as defined in Section 2.4. To meet this requirement, high capacity, energy dense batteries are required. The power delivery system for HADES needs to supply various components with their required voltages and currents and the power supplies need to be robust and reliable to support the varying and noisy loads. Most high-power density batteries require a battery management system to prevent damage to the battery and the connected electronics. The design and implementation of the power delivery system is covered in this section.

\subsubsection{Batteries}

Lithium-Polymer (Li-Po) and Nickel batteries are most desirable for compact mechatronic platforms due to their high charge density [30]. Nickel batteries are higher cost than Li-Po batteries (350 USD/kWh vs 125 USD/kWh), but have a higher number of charge/discharge cycles before the battery begins to degrade [30]. Li-Po batteries are more commonly available than Nickel batteries as they are commonly used in hobby drones and cars. Li-Po and Nickel batteries need to be charged in a flame proof, temperature controlled environment and hence they need to be removed from the HADES chassis for charging. As the batteries are can be removed, Li-Po Batteries are used as they are less than half the total price of a Nickel battery system and are easier to acquire.

The HADES batteries have two distinct power delivery tasks, power the locomotion systems and power the control and sensing electronics. Table 6.3 outlines the estimated current and voltage requirements for the control system and motor batteries. 
Table 6.3: Voltage and current requirements for the battery systems on HADES.

\begin{tabular}{|l|l|l|}
\hline & Locomotion Systems & Control System \\
\hline Continuous Current & $2 \mathrm{~A}$ & $1 \mathrm{~A}$ \\
\hline Peak Current & $200 \mathrm{~A}$ & $5 \mathrm{~A}$ \\
\hline Voltage & $20-26 \mathrm{~V}$ & $20-26 \mathrm{~V}$ \\
\hline
\end{tabular}

Li-Po cells have a nominal voltage of $3.7 \mathrm{~V}$, so to supply a $22 \mathrm{~V}$ system a sixcell (6S) Li-Po battery is needed. To supply a constant current of 2 A for 4 hours, a battery capacity of $8 \mathrm{Ah}$ is needed. The locomotion systems can draw up to 200 A for short periods of time (0.5-3 ms) during start-up. To allow for this, and to increase the safety factor, the capacity requirement is increased to a minimum of $10 \mathrm{Ah}$.

The maximum current a Li-Po battery can provide is defined by its $\mathrm{C}$ rating. Two $\mathrm{C}$ ratings are usually given, one for continuous current $\left(C_{c}\right)$ and one for peak current $\left(C_{p}\right)$. The $\mathrm{C}$ rating is related to the maximum current by,

$$
I_{\text {Max }}=C \cdot \text { Capacity }
$$

where $\mathrm{I}_{M a x}$ is the maximum current the battery can supply in A and Capacity is the maximum charge stored in the battery in Ah. For an 10 Ah hour battery to supply a constant $2 \mathrm{~A}$ it needs a minimum $C_{c}$ rating of 0.2 . To supply the peak current of $200 \mathrm{~A}$ the $C_{p}$ rating needs to be at least 20. The control system battery draws less continuous and peak current than the motor batteries, thus the same battery can be used to supply the motors and control system.

Li-Po batteries are explosive and subject to strict shipping regulations. For this reason, batteries could only be purchased from local suppliers. This limited the battery choice to a $6 \mathrm{~S}, 12$ Ah battery with a $C_{c}$ rating of 10 and a $C_{p}$ of 20 . This is acceptable as it exceeds all the requirements for both the motor and control system batteries, with the estimated operating time well in excess of 4 hours. 
The $12 \mathrm{~V}$ rail supplies a number of critical control components, such as, the NUC and the BMS relays (Section 6.2.2). To provide redundancy and reduce the load on the control system battery, an auxiliary battery is added. The auxiliary battery is a 3S, 8 Ah battery that has a voltage range between 11.1 and $12.4 \mathrm{~V}$. In normal operation the auxiliary battery is only used if the control system battery or the $12 \mathrm{~V}$ regulator are failing. The auxiliary battery can be connected directly to the $12 \mathrm{~V}$ rail to provide such redundancy. The implementation of the switching circuitry is covered in Section 6.2.3.

\subsubsection{Battery management}

Li-Po batteries can expand, heat up and even explode if unsafe operating conditions are reached. A battery failure could potentially damage the robot or even endanger rescue operations. To prevent a battery failure from occurring, a series of safety and monitoring devices are used.

Li-Po batteries have three main electrical failure modes, over current, under/over voltage and under/over temperature. There are also several mechanical failure modes; these are discussed in Section 3.1.6. Table 6.4 shows the limits of the safe operating conditions for the batteries used in HADES. If any of these conditions are breached, the battery could catastrophically fail or become damaged.

Table 6.4: The safe operating limits for the batteries used in HADES.

\begin{tabular}{|l|l|l|l|}
\hline Bat & Current & Voltage (min/max) & Temperature \\
\hline Motor and Control & $240 \mathrm{~A}$ & $18 / 27 \mathrm{~V}$ & $-10 / 35^{\circ} \mathrm{C}$ \\
\hline Auxiliary & $160 \mathrm{~A}$ & $9 / 13.5 \mathrm{~V}$ & $-20 / 40^{\circ} \mathrm{C}$ \\
\hline
\end{tabular}

To prevent these hazardous conditions, a battery management system (BMS) is used. A BMS monitors the temperature, cell voltages and current of the battery to determine its health. If the battery is unhealthy, the controller can then 
act to reduce the load or isolate the battery. There are two battery management system types used in HADES, one for the high current locomotion systems and one for the control and auxiliary batteries. The cell voltage and temperature measurement circuitry is similar in both systems.

The cell voltage of a Li-Po Battery can range between $1.7 \mathrm{~V}$ and $4.6 \mathrm{~V}$. However, the safe operating range is often regarded as being $3.0 \mathrm{~V}$ to $4.2 \mathrm{~V}$ [16]. The cells of a Li-Po battery need to remain balanced to prevent one cell from working harder than the others. However, as cells become damaged or degraded, the voltages of each cell may vary. The voltage of a cell is also dependant on the remaining charge in the cell. By monitoring the cell voltages, the health and remaining charge of the battery can be assessed.

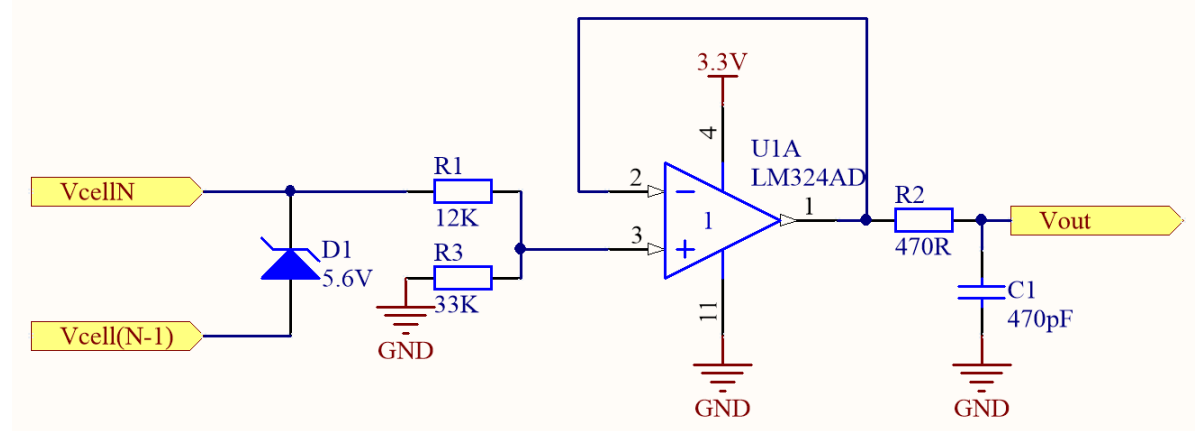

Figure 6.3: The circuity used to measure the voltage of a single cell.

To monitor the cell voltages, a resistor divider network is used to reduce the voltages before an ADC samples them. The voltage of each cell is divided using R1 and R3 (Figure. 6.3), where the values of the resistors are in the $10 \mathrm{k} \Omega$ to $1 \mathrm{M} \Omega$ range to minimise parasitic current draw from the battery. Table 6.5 shows the resistor values used for each cell. To protect the cell and the ADC, a Zener diode (D1) is used to clamp the maximum cell voltage.

To drive the high impedance input of the ADC, U1 is set up as a voltage follower to buffer voltage from the resistor divider. As the ADC is a largely capacitive load, R2 and C1 act as an impedance matching filter to prevent the 
Table 6.5: The resistor values used in the divider networks for cell voltage measurement.

\begin{tabular}{|l|l|l|l|l|}
\hline Cell Num & Max Voltage (V) & $\mathbf{R} 1 \mathbf{( k} \Omega)$ & $\mathbf{R} 2 \mathbf{( k} \Omega)$ & Vout $(\mathbf{V})$ \\
\hline 1 & 4.5 & 12 & 33 & 3.3 \\
\hline 2 & 9 & 62 & 36 & 3.3 \\
\hline 3 & 13.5 & 68 & 22 & 3.3 \\
\hline 4 & 18 & 160 & 36 & 3.3 \\
\hline 5 & 22.5 & 360 & 62 & 3.3 \\
\hline 6 & 27 & 130 & 18 & 3.3 \\
\hline
\end{tabular}

op-amp from oscillating. The cell voltages are sampled at $1 \mathrm{~Hz}$, thus the filter is designed to have a rise time of approximately $250 \mathrm{~ms}$. As high bandwidth measurements are not required, a low-cost LM324 Quad op-amp is used. Figure 6.3 shows the circuit used for measuring the voltage of a single cell, to measure all the cells in a battery, this circuit is repeated for each cell.

A Microchip MCP3208 eight channel ADC is used to sample the voltage outputs from the resistor divider network. The MCP3208 is a 12 bit ADC which provides a voltage resolution of $0.8 \mathrm{mV}$ when used with a $3.3 \mathrm{~V}$ reference voltage. This greatly exceeds the $10 \mathrm{mV}$ resolution that is generally accepted for cell voltage measurement [16]. For the control and motor batteries, six of the eight inputs of the ADC are used for cell voltage measurement; the remaining two are used for battery temperature and current measurement. In the case of the auxiliary battery BMS, five of the inputs are used to monitor the cell voltages, current and temperature of the battery. The remaining three ADC inputs are connected to temperature sensing devices to monitor the voltage regulators and distribution switches (Section 6.2.3).

To measure the temperature of the batteries, a PTC thermistor is used in a resistor divider configuration. An op-amp is used to buffer the signal before it is passed into the ADC. Like the cell voltage signals, the output of the op-amp has 


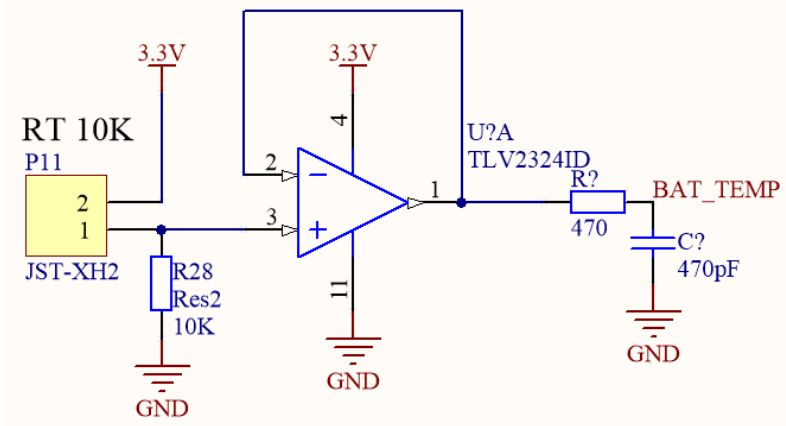

Figure 6.4: The temperature measurement circuitry for the battery system in HADES.

a low pass filter to prevent oscillation when driving the ADC. The thermistor is connected via $200 \mathrm{~mm}$ cables so it can sit directly against the battery. Figure 6.4 shows the temperature measurement circuit.

\subsubsection{Motor Battery Management System}

The $6 \mathrm{~S}$ batteries used for the ECS and motors can deliver up to 120 A continuously, with peak currents up to 300 A. The ESC can also regeneratively brake supplying up to $50 \mathrm{~A}$ back into the batteries. To accurately measure these currents, two measurement techniques were investigated; Hall Effect sensors and shunt resistors. Shunt resistors were quickly discounted as the power rating needed to pass the peak currents resulted in large packages and reduced resolution compared to Hall Effect sensors.

An Alegro ACS758-200B Hall Effect sensor is selected to measure the battery current for the motors. These sensors can measure \pm 200 A current and can provide an output voltage proportional to the current flow, centred around Vcc/2. When the sensors are powered with a $3.3 \mathrm{~V}$ supply, the output has a sensitivity of $6.6 \mathrm{mV} / \mathrm{A}$. When connected to the MCP3208 12 bit ADC, the current can be measured with a resolution of $120 \mathrm{~mA}$. The ADC reading $\left(N_{a d c}\right)$ can be related 
to the current $(I)$ by,

$$
I=\left(\frac{N_{a d c} V_{R e f}}{2^{n}}-\frac{V_{R e f}}{2}\right) \frac{1}{V_{\text {Sense }}}
$$

where $V_{\text {Ref }}$ is the ADC reference and sensor supply voltage, $n$ is the bit depth of the ADC and $V_{\text {sense }}$ is the sensitivity of the sensor. The current sensing circuitry for the motor BMS can be seen in Fig. 6.5.

The ESCs use MOSFETs to switch the currents in the motors. When MOSFETs fail they often fail to a short circuit [31]. If a failure was to occur, it is likely that both the motor and the battery would become damaged. To prevent this, the BMS system should be able to isolate the ESC from the battery.

To limit the short-circuit currents, a fuse could be used, however, if a fuse was to blow while on a rescue mission a robot could become stranded. Two alternative switching methods were investigated; relays and solid-state switches. Solid-state switches were found to be an order of magnitude more expensive and twice the size of a similarly rated relay. However, relays need a constant current to hold their state and relays can also weld shut if the voltage or current rating is exceeded.

Despite their shortcomings, relays are used for their lower cost and smaller footprint. Automotive grade CB1AH relays are chosen for their heightened vibration resistance. The relays are normally open and require a current of $100 \mathrm{~mA}$ at $12 \mathrm{~V}$ to close. The relays are rated to a constant current of $200 \mathrm{~A}$ and can disconnect up to $250 \mathrm{~A}$. To switch the relays, a low-side N-MOS switch is used. The gate of the MOSFET is connected to the power management microcontroller. To catch the back EMF from the relay, D1 is used as a flyback diode. Figure 6.5 shows the current measurement and switching circuitry used for the ECSs.

Two methods were investigated for distributing the power to the motors and ESCs; a single power bus and independent power bus method. The single power bus method has one power bus, which all the batteries and motors are 


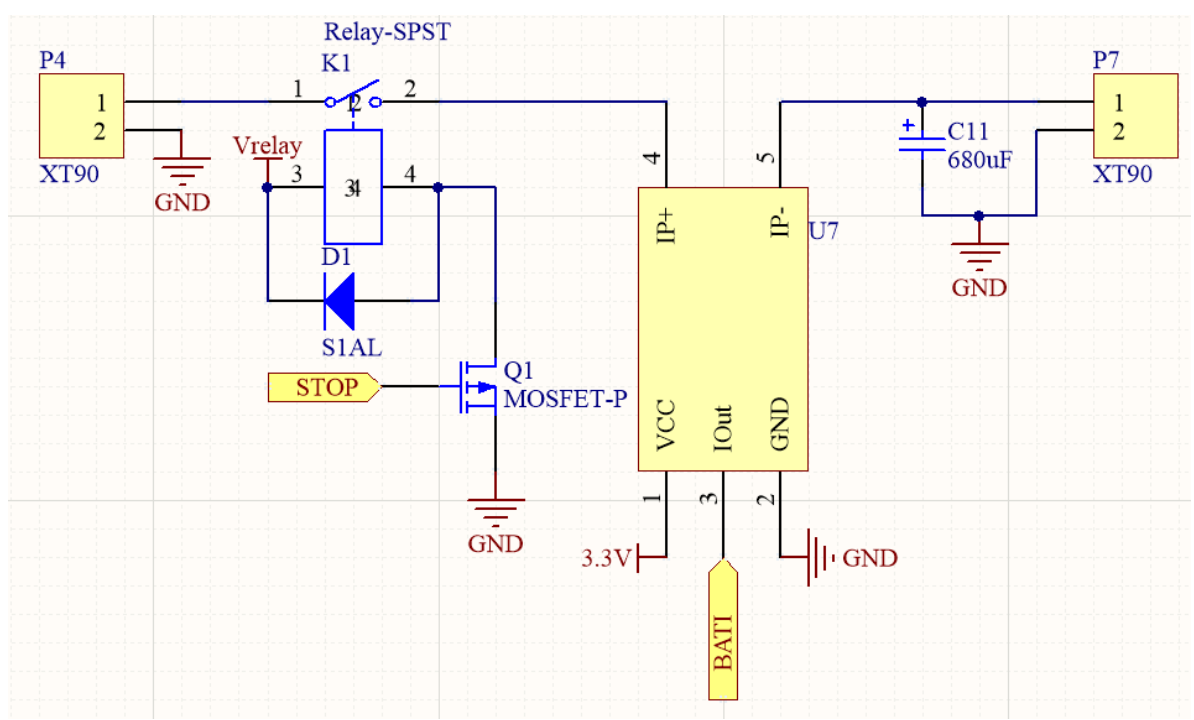

Figure 6.5: The current sense and switching system for the the motor battery BMS.

connected to in parallel. Each battery and ESC has a relay for isolating it from the power bus. The independent power bus system has a power rail for each motor and ESC, where each system is independent sharing a common ground. The two methods can be seen in Fig 6.6.

The single power bus method is more resilient to individual component faults as the rest of the system can compensate, but the power bus still allows a single failure to compromise the system. The A/NZ600798.11 standard states that Li-Po cells should not be connected in parallel to prevent cross charging. The single power method requires twice the number of relays needed for the independent power system, thus using more internal chassis volume and PCB space. Table 6.6 compares the two methods.

To meet the A/NZ60079.11 standard, the independent power bus method was chosen. To prevent accidental reverse connection, the batteries are connected to the power management PCB (Section 6.2.4) with XT90 connectors. The opposite gender connectors are used for connecting the BMS to the ESCs. 


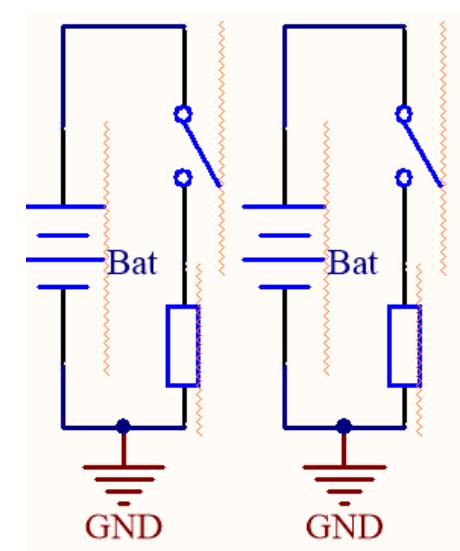

(a) The independent power bus distribution method.

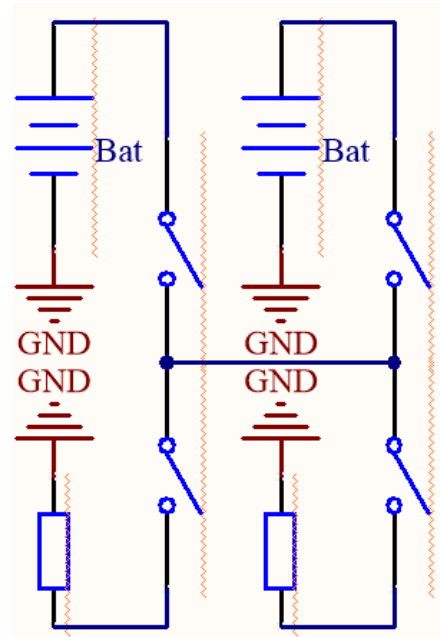

(b) The single power bus distribution method.

Figure 6.6: The two considered power distribution methods for the motor batteries in HADES.

Table 6.6: A comparison of the two power distribution methods for the motor batteries.

\begin{tabular}{|l|l|l|}
\hline & Independent Power Bus & Single Power Bus \\
\hline Relays needed & 4 & 8 \\
\hline Failures for complete failure & 4 & 1 \\
\hline Failure compensation & No & Yes \\
\hline A/NZ60079.11 approval & Yes & No \\
\hline
\end{tabular}

\subsubsection{Control and Auxiliary Battery Management Systems}

The control and auxiliary batteries supply significantly less current than the motor batteries, so a smaller range, higher resolution ACS712-30A Hall Effect sensor is used. These sensors have a $0 \mathrm{~A}$ to $30 \mathrm{~A}$ range and a proportional 0 to $3.3 \mathrm{~V}$ output. Using an external capacitor, the bandwidth of these sensors 
can be set. For the battery measurement, a $1 \mathrm{nF}$ capacitor is used, setting the bandwidth to $220 \mathrm{~Hz}$ [4]. Figure 6.7 shows the current sensor setup for the auxiliary and control batteries.

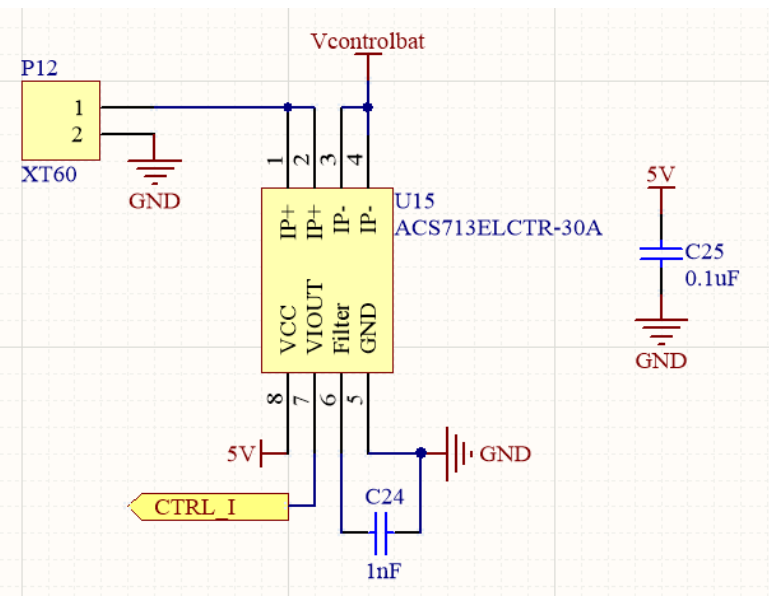

Figure 6.7: The Hall Effect sensor used for measuring the current from the control and auxiliary batteries.

Unlike the motor batteries, the control and auxiliary batteries should never be disconnected from the system as this will shut the system down. Fault conditions are instead managed by the power regulation circuitry. The regulation circuitry allows faulty sub-systems to be removed from the supply rail without compromising the complete distribution system. The auxiliary and control system batteries are connected to the power PCB with XT60 connectors. The auxiliary BMS system has an output XT60 connector for supplying power to any auxiliary components (communication node dispensers / other chassis addons).

\subsubsection{Regulated Supplies and Monitoring}

The components of HADES have differing power supply requirements, if any component loses power it will fail and so a robust and monitorable power de- 
livery system is needed. There are three main supply voltages needed for the electronics in HADES: $3.3,5$ and $12 \mathrm{~V}$. As all the sub-systems need to communicate using I2C, SPI or CAN-BUS, a common system ground is needed. As maximising battery life is a requirement, DC-DC converters should be used where the application permits.

To track battery usage and monitor regulator stability, the power controller should be able to monitor the supply rails. The power controller should be able to track the current draw of key system components and shut them off if necessary. As a fault in the power deliver system could cause a system wide failure, key power delivery components should be redundant.

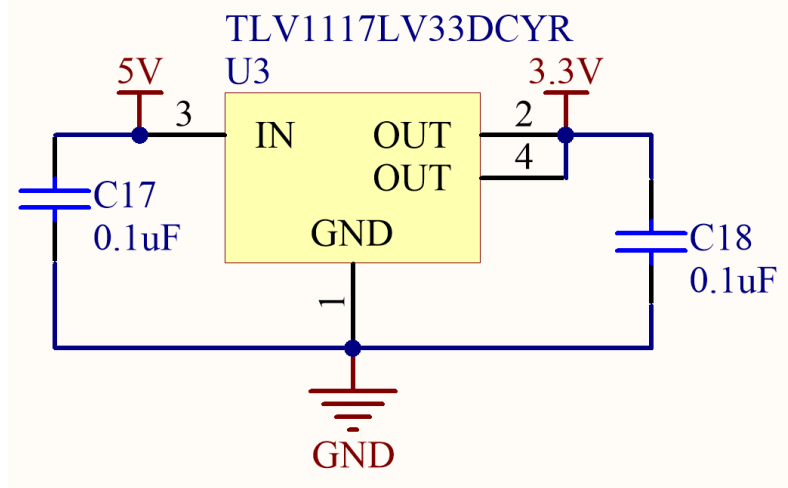

Figure 6.8: The TLV1117LV33 LDO regulator used for supplying 3.3 V to each of the sub-systems

The 3.3 $\mathrm{V}$ rail supplies all the digital and analogue electronics. The analogue electronics are sensitive to power supply noise. To isolate the noise between sub-systems and provide supply redundancy, each sub-system is powered with a $3.3 \mathrm{~V}$ linear regulator. A linear regulator is used as it rejects the switching noise from the $5 \mathrm{~V}$ switching regulator. Figure 6.8 shows the TLV1117LV33 LDO regulator used in each of the sub-systems.

As the $3.3 \mathrm{~V}$ regulation is handled by each sub-system, the power delivery system needs to only supply $5 \mathrm{~V}$ and $12 \mathrm{~V}$. Table 6.7 shows the estimated con- 
tinuous and peak current requirements for the $12 \mathrm{~V}$ and $5 \mathrm{~V}$ rails respectively. As the regulators will be supplied by the control and auxiliary batteries, they will need function over a voltage range of $10 \mathrm{~V}$ to $27 \mathrm{~V}$.

Table 6.7: The power requirements for the $5 \mathrm{~V}$ and $12 \mathrm{~V}$ supply Rails.

\begin{tabular}{|l|l|l|l|}
\hline & Component & Continuous Current & Peak Current \\
\hline 5 V Rail & Power PCB & $200 \mathrm{~mA}$ & $400 \mathrm{~mA}$ \\
\hline & Main PCB & $100 \mathrm{~mA}$ & $300 \mathrm{~mA}$ \\
\hline & Aux PCB & $100 \mathrm{~mA}$ & $200 \mathrm{~mA}$ \\
\hline & & & \\
\hline 12 V Rail & NUC & $1 \mathrm{~A}$ & $3.5 \mathrm{~A}$ \\
\hline & BMS relays & $400 \mathrm{~mA}$ & $400 \mathrm{~mA}$ \\
\hline & Sensor System & $100 \mathrm{~mA}$ & $500 \mathrm{~mA}$ \\
\hline
\end{tabular}

\subsubsection{5 V Supply}

The $5 \mathrm{~V}$ rail is supplied by an Recom 78B5-2.0 switching regulator, capable of supplying $2 \mathrm{~A}$. To provide supply redundancy, two regulators are used, one is powered from the control system battery and one is powered from the auxiliary system battery. The power controller can switch between the two regulators using the TPS2110A power management switch [73]. This switch can switch between supplies in $60 \mu \mathrm{s}$. To prevent brown-outs during this switching period, a reservoir capacitor is needed, which is calculated using Eq. 6.5.

$$
C=\frac{I \cdot t_{\text {switch }}}{V_{\text {droop }}}
$$

$V_{\text {droop }}$ is the allowable voltage droop, $I$ is the maximum current, $t_{\text {switch }}$ is the time taken to switch and $C$ is the required capacitance. The TLV1117LV33 regulators used for the $3.3 \mathrm{~V}$ supply will stop regulating at $3.9 \mathrm{~V}$, this allows for 
a maximum voltage drop of $1.1 \mathrm{~V}$ from the $5 \mathrm{~V}$ rail, and at maximum current, the $5 \mathrm{~V}$ rail will be supplying $2 \mathrm{~A}$. Using Eq. 6.5 the output capacitor should be greater than $180 \mu \mathrm{F}$. As this is a minimum requirement, a $680 \mu \mathrm{F}$ capacitor is used. The TPS2011A also limits the supply current with inbuilt current clamps. The current limit is set with R59 (Figure. 6.9), the value of which is calculated by $R_{\text {lim }}=250 / I_{\max }$ [73]. To limit the current to 2 an $82 \Omega$ resistor is used. Figure 6.9 shows the supply and switching circuity for the $5 \mathrm{~V}$ supply rail.

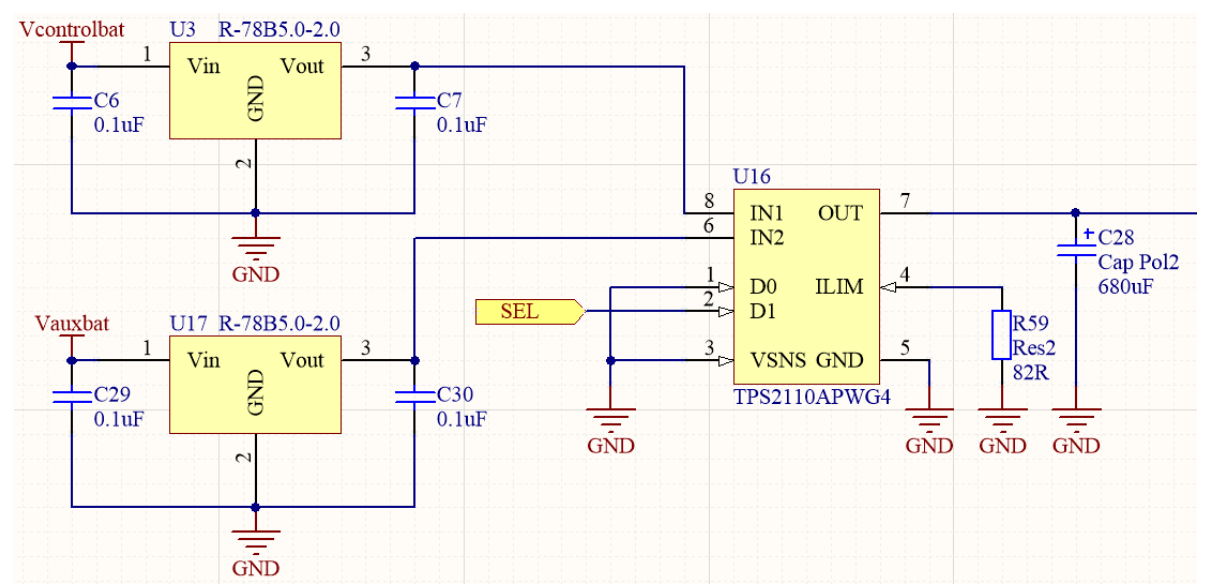

Figure 6.9: The $5 \mathrm{~V}$ regulators and TPS2110A power management switch used for the $5 \mathrm{~V}$ supply rail in HADES.

To track the stability of the $5 \mathrm{~V}$ rail, the voltage is monitored with a resistor divider and an ADC in the power controller. The resistor divider reduces the $5 \mathrm{~V}$ signal so it does not overload the $3.3 \mathrm{~V}$ ADC. The current output of the regulator is monitored with the ACS723-05A Hall Effect sensor. Both the current and voltage are measured by the power controller and can be used for calculating the output power of the $5 \mathrm{~V}$ regulator. If the output power exceeds a predefined threshold, the software system can issue shutdown commands to the sub-systems to reduce the load (Section 8.3). Figure 6.10 shows the monitoring circuity for the $5 \mathrm{~V}$ regulation stage. 


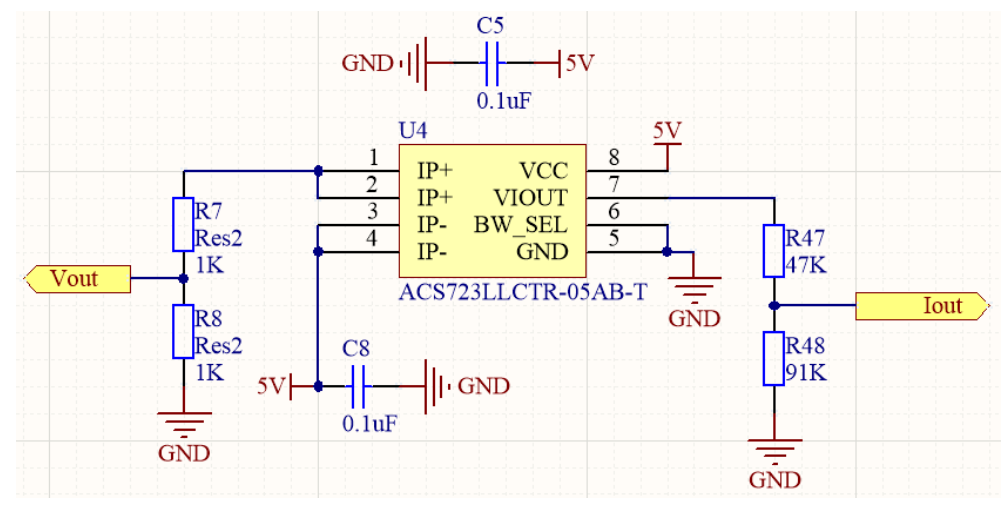

Figure 6.10: The resistor divider and hall effect sensor used for monitoring the output from the $5 \mathrm{~V}$ regulators

\subsubsection{12V Supply}

The primary $12 \mathrm{~V}$ supply is generated using a LM2678S-12 switching regulator capable of supplying $5 \mathrm{~A}$. This supply is used primarily for supplying power to the NUC, audio system and sensor system. The NUC is a critical component and is also the highest power control component. For this reason, the NUC needs to have a redundant supply and an independent monitoring system. The NUC has been shown to function at voltages down to $10.8 \mathrm{~V}$, and so it can be powered directly from the $3 S$ auxiliary battery, provided the battery voltage is above $10.8 \mathrm{~V}$. The minimum operating voltage of the audio system and the external sensors is $8 \mathrm{~V}$, thus they will be unaffected by the voltage drop.

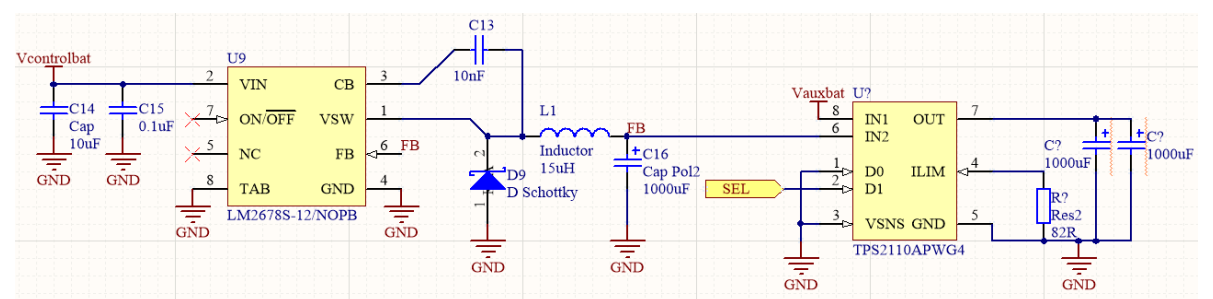

Figure 6.11: The LM2678S regulator and TPS2011 power management switch used for the $12 \mathrm{~V}$ supply rail. 
A TPS2011 power management switch is used to switch between the $12 \mathrm{~V}$ regulator and the auxiliary battery. Using Eq. 6.5, the output capacitor is increased to $2000 \mu \mathrm{F}$, to allow for the increased current draw. The limiting resistor is also updated to $51 \Omega$ to set the current limit to 4.9 A. Figure 6.11 shows the LM2678 regulator and power management switch.

The $12 \mathrm{~V}$ rail is monitored with a similar resistor divider configuration to the $5 \mathrm{~V}$ rail. An ACS723-10A Hall Effect sensor is used to monitor the current supplied by the regulator. The NUC does not have inbuilt power switch capability, and therefore an external switch is needed to disconnect it from the $12 \mathrm{~V}$ rail. To prevent from creating a floating ground, a high-side switch is needed.

A TPS2556 high-side power management switch is used, the current limit of switch is set with a resistor. The value of the resistor is given by,

$$
R=\left(\frac{99038}{I_{\max }}\right)^{\frac{1}{0.947}}
$$

where $R$ is the resistance and $I_{\max }$ is the maximum current [74]. The NUC should draw no more than $3.5 \mathrm{~A}$, so the current limit is set with a $34 \mathrm{k} \Omega$ resistor. When the current limit is exceeded, the TPS2556 will enter the constant current mode and pull the fault line low. Figure 6.12 shows the high-side switch and the Hall Effect sensor used for monitoring the power supply of the NUC.

\subsubsection{Other Supply Rails}

The external sensors require additional $-5 \mathrm{~V}$ and $4.25 \mathrm{~V}$ supplies that are not used in other sub-systems. As the supplies used for the sensor pod need to meet the intrinsic safety standard, the sensor pod should have independent $5 \mathrm{~V}$ and $3.3 \mathrm{~V}$ rails. When travelling through extremely explosive atmospheres, the sensor pod should be isolated from power. As some of the sensors will still produce current without a power supply, the external sensor pod should remain grounded, thus a high-side switch is needed. 


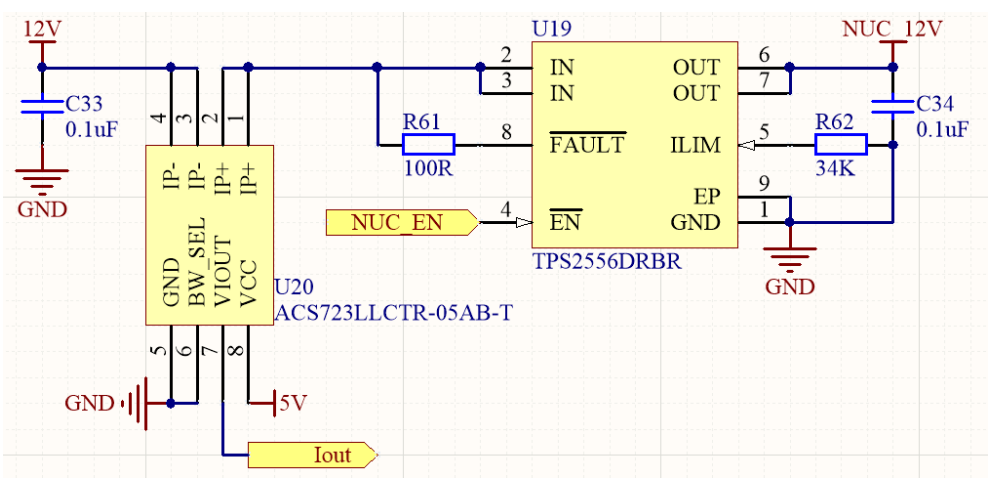

Figure 6.12: The high side current switch and hall effect sensor used for monitoring/controlling the power supply of the NUC.

The external sensor pod has sensitive analogue electronics for detecting the external gas concentrations. These electronics need a noise-free power supply to provide accurate readings (Section 5.2.3). Linear regulators have a much higher noise rejection ratio than switching regulators and they also do not produce any switching noise and hence should be used for supplying the external sensor pod. All the power supplied to the external sensor pod needs to pass though intrinsic safety barriers as discussed in Section 5.2.2.

The battery system on HADES does not produce any negative voltage supplies, so one needs to be generated for the $-5 \mathrm{~V}$ rail. The $12 \mathrm{~V}$ supply is inverted using the LM2776 switched charge inverter [75]. To remove the switching noise, the $-12 \mathrm{~V}$ signal is regulated by a LM7905 linear regulator. Figure 6.13 shows the charge inverter and the linear regulator used for the $-5 \mathrm{~V}$ supply rail.

The explosive gas sensor (Section 5.2.1) requires a $4.25 \mathrm{~V}$ supply for correct operation. As a fixed $4.25 \mathrm{~V}$ regulator cannot be purchased, the $4.25 \mathrm{~V}$ rail is generated with a LM319 adjustable linear regulator. Before the external sensor pod is connected, the output voltage of the regulator will have to be set manually using R25 (Figure. 6.14).

To isolate the $12 \mathrm{~V}$ supply for the external sensor pod regulators, a TPP2556 


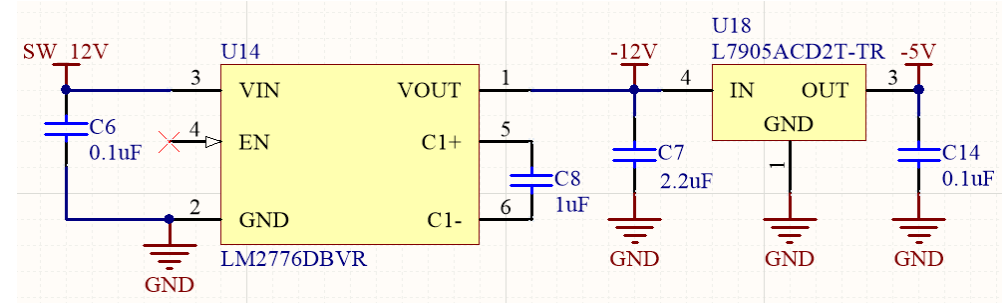

Figure 6.13: The charge inverter and the linear regulator used for the $-5 \mathrm{~V}$ supply.

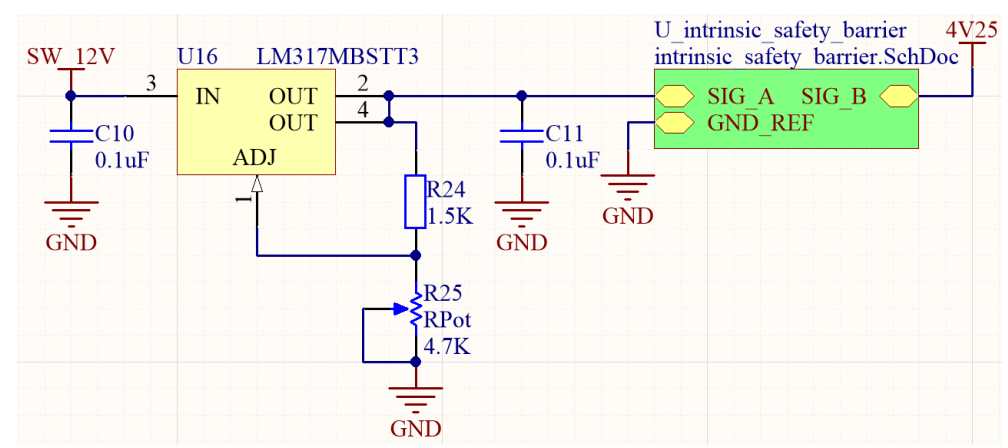

Figure 6.14: The $4.25 \mathrm{~V}$ regulator and intrinsic safety barrier for the explosive gas sensor.

high-side power management switch is used. The current limiting feature is used to manage the total output from the regulation system and is set to $600 \mathrm{~mA}$ using a $221 \mathrm{k} \Omega$ resistor (Eq. 6.6). The fault detect line of the power management switch is connected to the main microcontroller to help detect and manage faults in the sensor system. Figure 6.15 shows the power management switch for the external sensor pod.

\subsubsection{Power PCB}

The power PCB distributes the power to all the system components and acts as a mounting point for all the regulation and monitoring circuity. The high current supplies for the motors will interfere with the battery management sys- 


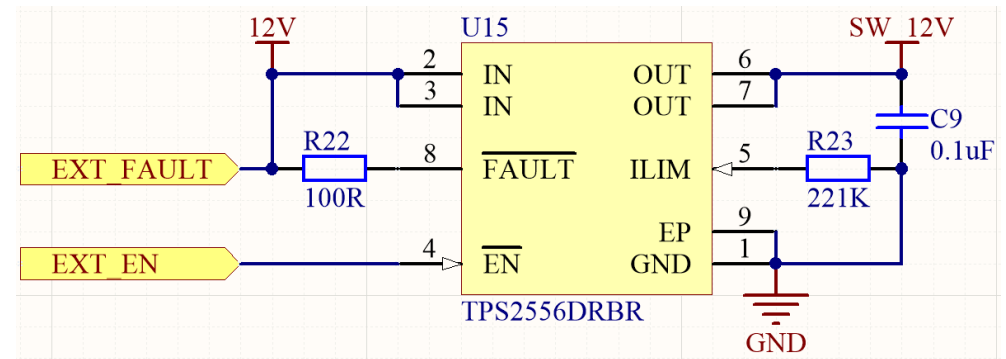

Figure 6.15: The power management switch for the external sensor pod.

tems if care is not taken to isolate them. The PCB shape is defined by the free space on the bottom hatch, limiting the PCB to a surface area of $150 \mathrm{~cm}^{2}$, thus limiting the separation between the high-power and analogue signals. To avoid interfering with components on the top hatch, the height of the components on the PCB is limited to $35 \mathrm{~mm}$. The power PCB can be seen in Figure. 6.16

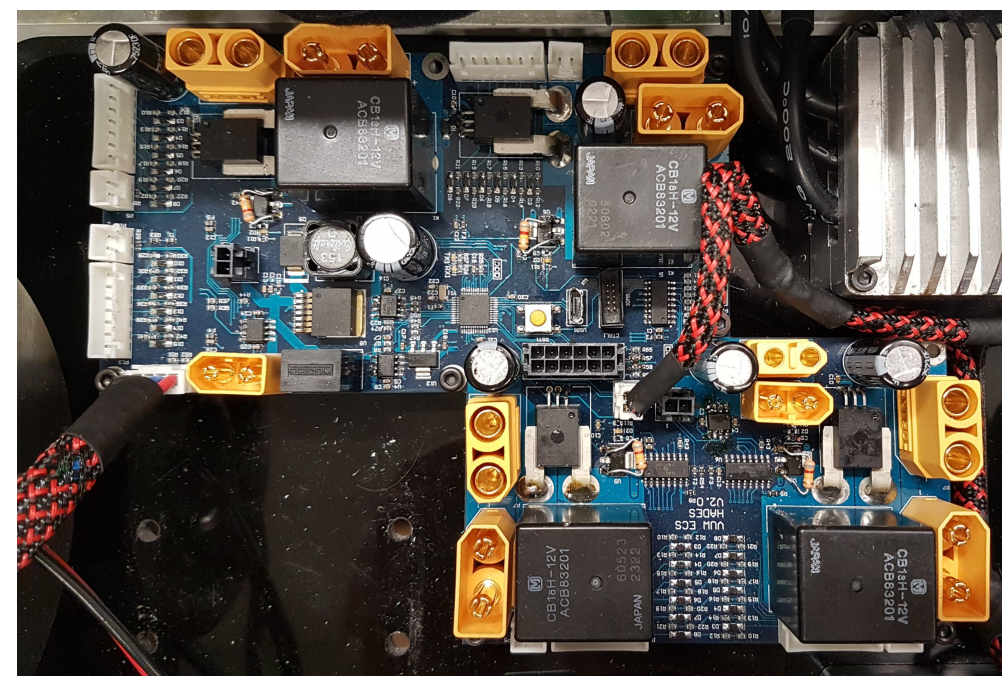

Figure 6.16: The completed power PCB mounted to the bottom hatch of HADES. 


\subsubsection{High Power Routing}

The high current needed for the motors needs special routing consideration as the high currents could cause the traces to increase in temperature or melt. The high currents will also produce EMF noise that could interfere with the operation of the microcontroller. The width of a trace $(W)$ for a given current $(I)$, copper thickness $(d)$ and temperature increase $(\Delta t)$ is given by,

$$
A=\left(\frac{I}{(k \Delta t)^{b}}\right)^{\frac{1}{c}}
$$

and,

$$
W=\frac{A}{1.378 d}
$$

where $k, b$ and $c$ are constants related to the fitting of the IPC-2221 curve [3]. The chassis temperature should not exceed $50{ }^{\circ} \mathrm{C}$, therefore at a $30^{\circ} \mathrm{C}$ ambient temperature, the maximum allowable temperature increase $(\Delta t)$ is $20{ }^{\circ} \mathrm{C} .1 \mathrm{ozft}^{-2}$ copper used for the PCB as it allows for smaller copper features needed for the analogue electronics. As all the high power traces are on external layers to improve thermal dissipation $k=0.024, b=0.44$ and $c=0.725$ from the IPC-2221 standard [3].

The majority of the high currents are passed using planes rather than traces as they can dissipate much more thermal energy. The power planes are duplicated on the top and bottom layers to increase the current carrying capability. Using Eq. 6.7 and 6.8 the minimum width of the planes was calculated to be $5 \mathrm{~mm}$. Unlike the low power components, the planes connect directly to the leads of the high power components. Figure 6.17 shows the power planes used to route the high power signals for the front left motor.

To isolate the high current stages from the power controller, they are located at the peripheries of the power PCB. The power planes used for routing the high power signals are routed so they maintain at least $5 \mathrm{~mm}$ of clearance to all other traces. Figure 6.16 shows the completed power PCB. 


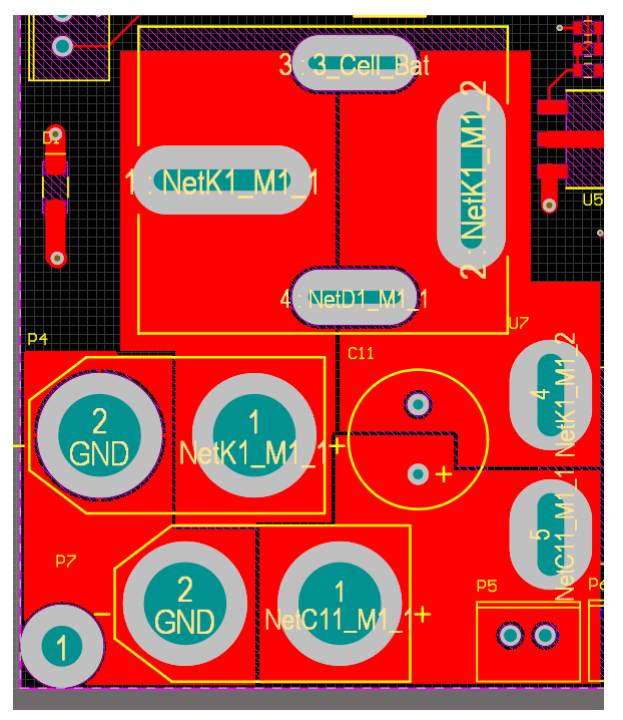

Figure 6.17: The power planes used to route the high current traces for the front left motor and ESC.

\subsubsection{Summary}

HADES is powered by six Li-Po batteries, each monitored by an independent battery management system (BMS). The BMS measures the currents, temperature and cell voltages of each battery to monitor their health and remaining charge. The motor BMS systems have a shut-off relay to isolate the drive system from the battery in fault conditions. The other system components are powered with two supply rails $(5 \mathrm{~V}$ and $12 \mathrm{~V}$ ). The regulation systems for each of the supply rails is doubly redundant, with the power controller switching between the two regulators. TO allow the power can easily be distributed to all the system components, the power management and distribution electronics are centrally located on the power management PCB. 


\section{Chapter 7}

\section{Software - ROS Control}

In normal operation, the Intel NUC is the highest level controller in HADES. The NUC needs to coordinate with the communication system, the hardware system and the five USB cameras used for video feedback to the operator. As well as the software on the NUC in HADES, software is needed on the base station to provide a control interface and display robot telemetry.

\subsection{Robot Operating System}

Coordinating the many aspects of a mechatronic system can be a complex task. To simplify this, a Robotic Development Environment (RDE) is often used to provide an abstraction layer between the hardware system and the high-level controllers. RDEs often contain complex libraries that allow the programmer to quickly implement many of the basic functions needed for robotic development.

ROS (Robot Operating System) is an RDE that runs on the Ubuntu operating system and is designed to act as a middleware between the controllers and the 
sensors/actuators [62, 24]. ROS is a large scale open source project originally developed at Stanford University but is now developed by many robotic labs around the world. These many contributors have produced packages that cover most common aspects of robotic functionality (computer vision, 3d mapping, navigation etc.).

ROS uses the TCI/IP network protocol to communicate between different nodes, a single processor can run multiple nodes, or they can be distributed over many processors with a TCI/IP network link. Each node is an independent process, inter-node communication is primarily completed with messages. A message is a data type that is comprised of generic (int, float, string) and ROS data types (map, point cloud, image). Nodes publish messages on a topic, other nodes can then subscribe to the topic to receive the information. When a node publishes a message, there is no feedback to confirm that the message has been received.

An ROS service is used when a request-response format is needed. Like a message, the service-call is comprised of basic and ROS data types. However, a service call must detail both the request and response formats. Services are less common than message calls as they are only used for an on-demand connection, while messages are used for a continuous data stream. A node or topic can also have parameters (i.e. video output resolution); parameters are registered with the parameter server. Any node can access the parameter server to modify the parameters of any other node or topic. The parameter server can also be accessed with the rqt_reconfigure graphical tool.

ROS nodes, messages and other datasets can be grouped together in a package to allow for easy distribution. A package typically will implement the code for one functional block of a robot (i.e. locomotion control), multiple packages are then used to implement the full functionality of a robot. ROS is not programming language specific. However, there is better support for $\mathrm{C}++$ and Python. While either of these could be employed, there is significantly greater 
institutional experience with $\mathrm{C}++$, and hence this was used as the developmental programming language.

Table 7.1: The names and functions of the ROS packages used in HADES.

\begin{tabular}{|l|l|}
\hline Package & Functionality \\
\hline HADES Video & Camera Drivers, Video multiplexer and compression \\
\hline HADES Locomotion & Kinematics and Drive control \\
\hline HADES Fault System & Fault Management and Reporting \\
\hline HADES Msgs & Message Definitions \\
\hline HADES Main & Global Launch Files \\
\hline HADES GUI & Graphical User Interface (GUI) for control \\
\hline
\end{tabular}

The ROS control architecture has been divided into six packages to complete all the functions required for the successful operation of HADES. Each package contains the required nodes, launch files and auxiliary data needed for one software function. To ensure the message definitions are constant throughout the system, all the custom message definitions are stored in the hades_msgs package. Table 7.1 lists the package names and functionality.

There are 21 custom messages for HADES, these are defined in their respective .msg files. When ROS is compiled the msg file is compiled, to a $\mathrm{C}++$ header file. These headers can then be shared between the different control packages. Listing 7.1 shows the definition for the motor control message (motor_ctrl.msg).

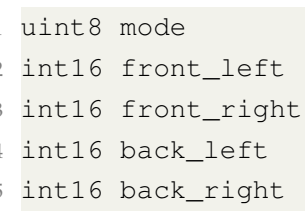

Listing 7.1: The message definition for the motor_ctrl.msg message. 


\subsubsection{Fault Codes}

HADES is teleoperated which means that HADES may operate in an area that is inaccessible for humans to undertake robot maintenance/repairs. The implication of this is that HADES must have a robust fault handling system that can manage, report and potentially solve faults without operator intervention. The fault system should be standardised throughout all levels of the HADES control architecture.

To report faults in a standard fashion, a fault is reported with three 8-bit codes. The first is the ID number of the system that reported the fault, the second is the error code, and the third is any extra information. In the hardware system, fault codes are transmitted over the I2C bus, the main microcontroller then publishes them to ROS. A fault handler attempts to solve faults that can be automatically remedied. Two fault handlers are implemented, one on the main microcontroller (Section 8.2) and one as a ROS node on the NUC.

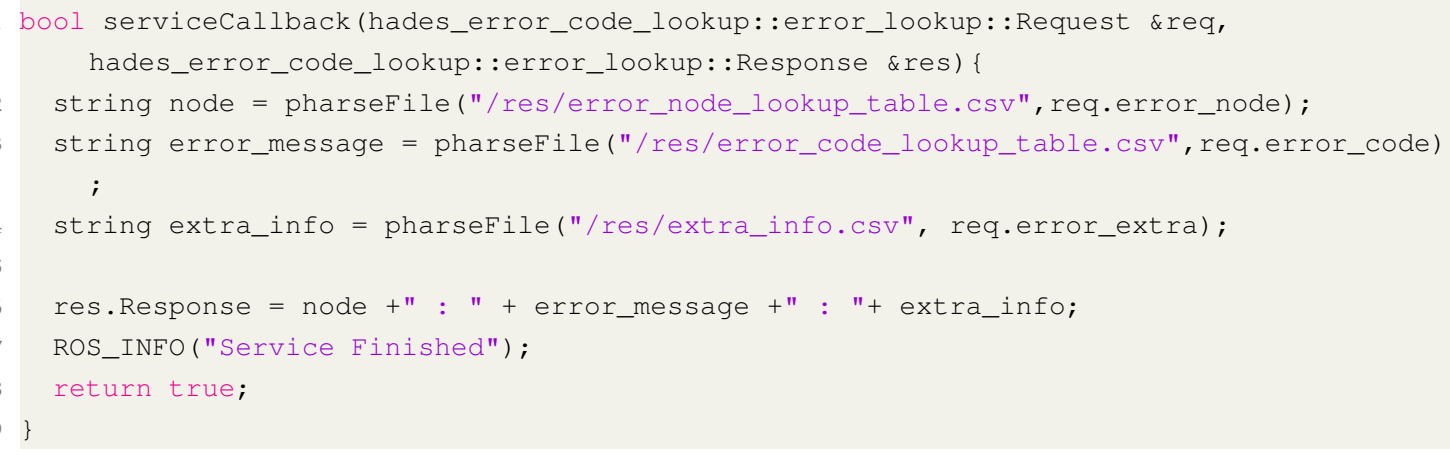

Listing 7.2: The service callback for converting a fault code into a human readable string

For the operator to interpret the error codes they must be converted into text-based strings. The string interpretations of the codes are stored in lookup tables which are stored as Comma Separated Variable (CSV) files. A ROS 


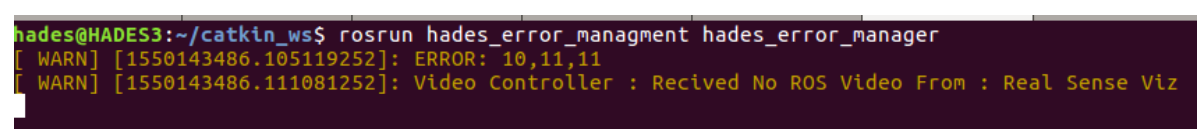

Figure 7.1: An example error code call from the video controller that has received no data from the Realsense camera.

service is used to convert the numerical codes to strings. The fault_code lookup service searches the CSV file for the appropriate line and returns the string. Listing 7.2 shows the service callback used to generate the string descriptions from the fault codes. The service callback uses the pharseFile() method to search the CSV files. The pharseFile() iterates through each line of the CSV until the correct code is found. When the line number of the code is known, the second cell on the line (which holds the string) description is returned.

Figure 7.1 shows an example error code report, where the video node has received no data from the front camera. To handle this error the ROS based fault handler will restart the front camera node (Section 7.1.2), the operator will also be notified via the communications link.

\subsubsection{Video Controller}

Video feedback is needed for the operator(s) to successfully navigate HADES though the underground mine. As discussed in Section 5.3, there are five cameras located around the chassis for different views of the surroundings, each of these cameras are connected to the NUC with a USB link. Camera drivers are needed to interface with the cameras and receive the video streams.

It is expected that the communication system will only have enough bandwidth to support the transmission of one compressed video stream, thus a video multiplexer and compression algorithm is needed. In addition to transmitting one stream, the video system should save full resolution streams from each camera to the local SSD. Saving the video data allows for later analysis 
after a successful entry. To save power, the video controller also needs to automatically switch the illumination LEDs (which are located around the chassis) on and off depending on which camera is selected.

To communicate with the SR300 Real Sense camera, the realsense2_camera node is used. This node produces three image streams; depth, infrared (IR) and visual. The realsense2_camera node also allows the operator to control the exposure, white balance and other camera properties. The realsense2_camera node also performs the image transform to register the IR, depth and visual camera feeds to a single frame.

To interface with the remaining cameras, the $u v c \_n o d e$ is used. The $u v c \_n o d e$ a node that uses the UVC camera driver to interface with most generic webcams. Like the realsense2_camera node, the $u v c \_n o d e$ also allows the operator to change the camera parameters with the parameter server.

Four instances of $u v c \_n o d e$ are used, one for each of the side and rear looking cameras and one for the forward looking thermal camera. Each camera appears in Ubuntu as a /dev/videoN device. Upon start-up Ubuntu assigns different cameras to different video devices, this causes the camera feeds to be randomly assigned to different topics (e.g. the thermal camera feed could be published on the uvc/image_raw topic). To prevent this from happening, each camera's serial number is locked to a particular video device by modifying a the /etc/udev/rules.d/10-local.rules file.

The side looking cameras have $180^{\circ}$ fish-eye lenses and so the image is very distorted around the edges. To correct for this distortion, the ROS image proc package is used. This package takes the camera distortion matrix and uses openCV to remove the fish-eye effect from the lenses. Two image proc nodes are used, one for each side camera. Both the raw and corrected camera streams are published to the video multiplexer. When the output from the image proc node output is not used by the multiplexer, the image correction is paused to reduce CPU load. Figure 7.2 shows the raw and corrected side camera images. 


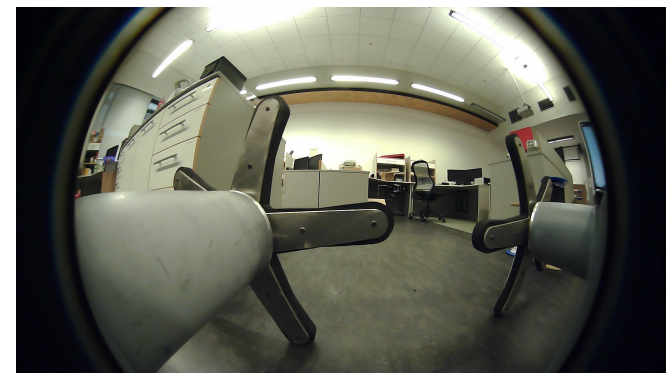

(a) Raw side camera image

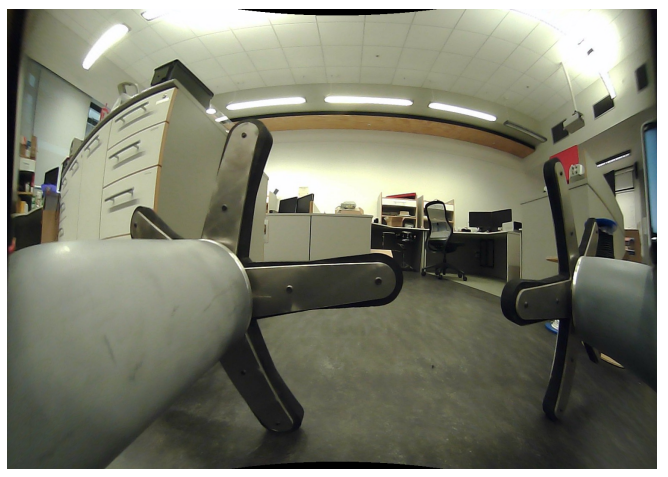

(b) Corrected side camera image

Figure 7.2: A side by side comparison of the raw and corrected images from the side cameras.

To select between the video streams, a bespoke video multiplexer was written. The video multiplexer subscribes to the video_ctrl message published by the base station. Based on the information from the base station, the correct camera stream is selected and re-published. The video_ctrl message also contains the settings for the video compression and frame rate.

To minimise CPU utilisation when the multiplexer is not using a video stream, the multiplexer unsubscribes from the incoming topic. A watchdog timer is used to monitor the incoming video stream if no frames are received for $2 \mathrm{~s}$, a fault code is generated. Listing 7.3 shows the callback for the video_ctrl message.

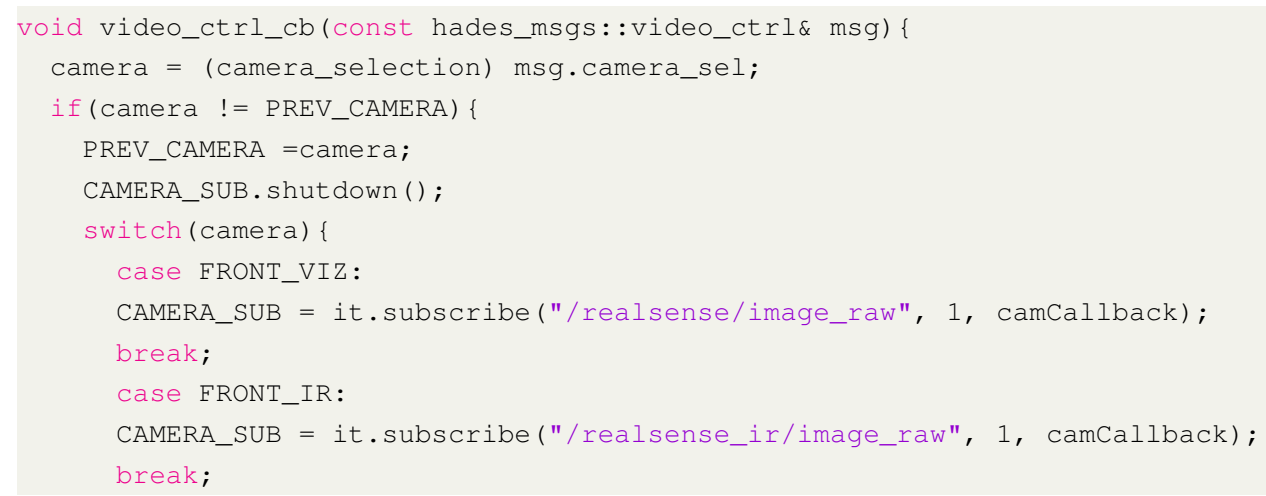




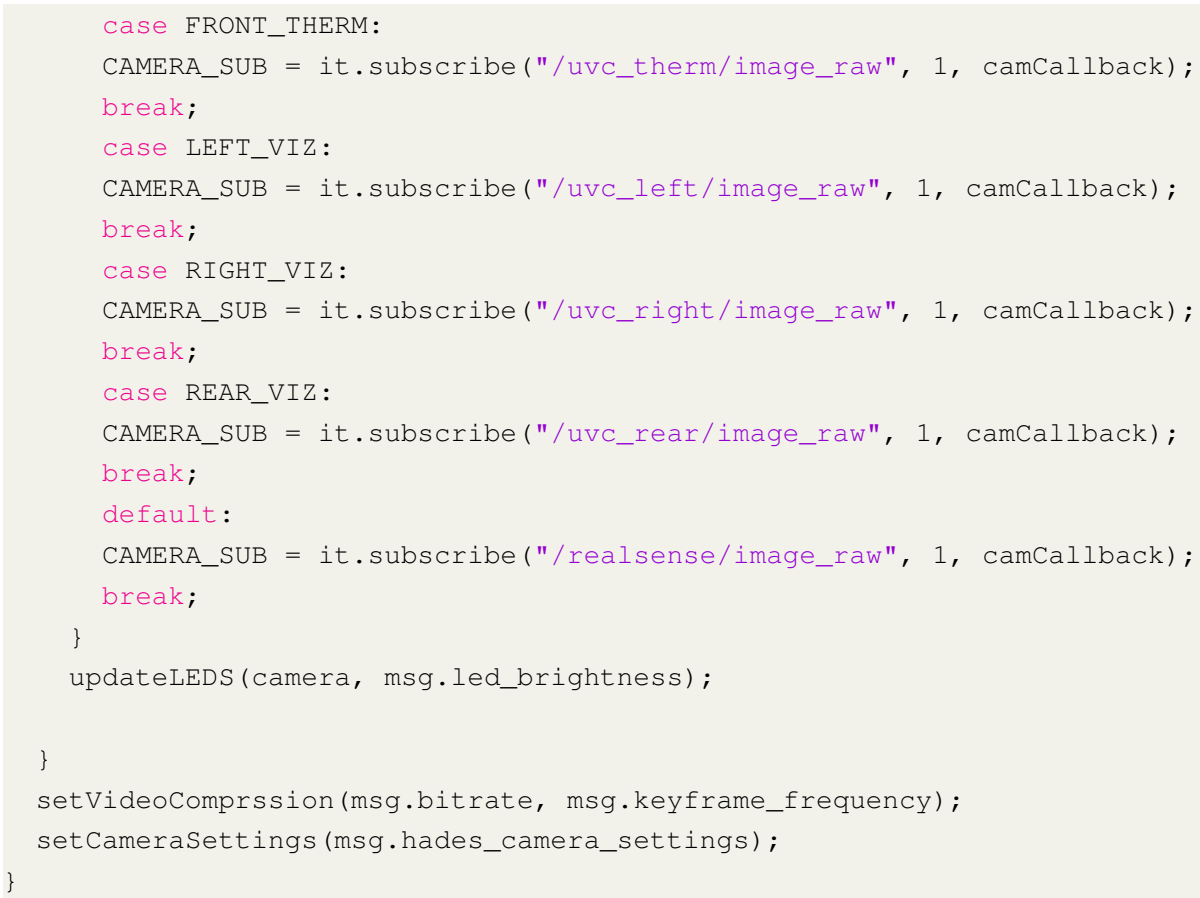

Listing 7.3: The ROS callback for the video_ctrl message.

In Listing 7.3 the video_ctrl message is received from the base station. If the camera varies from the currently selected camera, the subscriber is stopped (CAMERA_SUB.shutdown()). Based on the new camera selection, the subscriber is then restated using the it.subscribe() function. The led_ctrl message is then published using the updateLEDS() to set the intensity levels on the illumination LEDs around the chassis based on the selected camera. Finally the video compression and camera settings are updated using the setVideoComprssion() and setCameraSettings() functions.

ROS nativity uses the image_transport message type for the passing of images/video between nodes. The image transport intrinsically supports lossy (JPEG) and lossless (PNG) image compression methods. JPEG and PNG are designed for compressing individual images, not a video stream and so they will be using more bandwidth than a dedicated video compression method. 
To use a video compression method, the theora_image transport plugin is used. This allows the video stream to be compressed with the Theora image compression algorithm. The hades_video node publishes the theora_image_transport to the communication system.

To configure the compression settings, the ROS parameter server is used. Using the setParam() method, the hades_video node can change the parameters of the theora_image_transport, thus changing the compression settings. The frame rate and image size is changed in the same way. Listing 7.4 shows the code for changing the compression settings of the theora_image_transport.

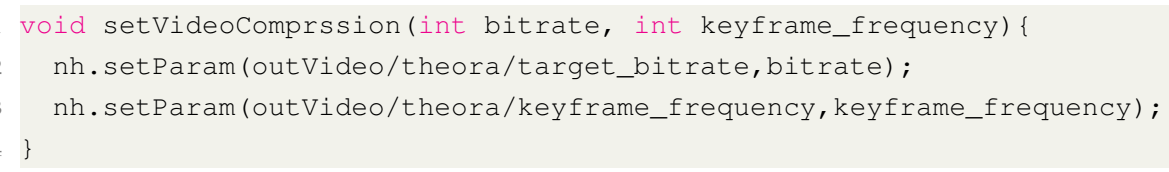

Listing 7.4: The function for setting the compression settings of the theora_image_transport using the parameter server.

The Theora video compression algorithm has two input parameters; the target bit-rate and the keyframe frequency. The target bit-rate adjusts the expected target bit-rate of the compressed video. The key-frame frequency parameter, adjusts how frequently the Theora compression publishes a keyframe.

The video system of HADES contains nine nodes; these interface with the cameras, select the correct image stream and compress the image. A block diagram of these nodes can be seen in Figure 7.3. The video is saved to the SSD on the NUC with the ros_bag package. This saves all the active ROS topics to a single time-stamped file. 


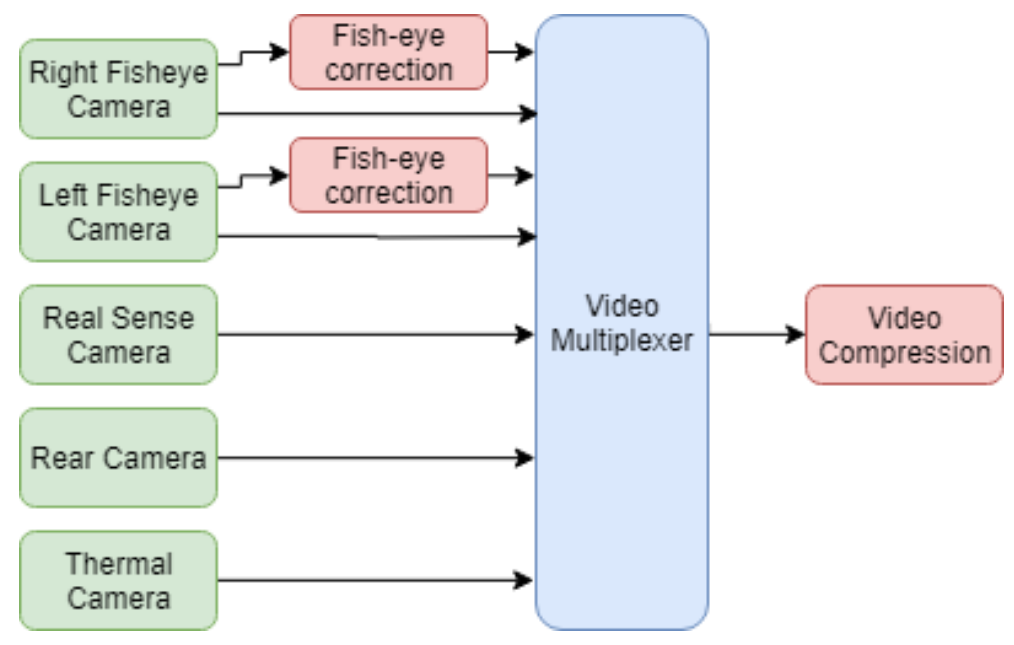

Figure 7.3: The block diagram for the video system in HADES.

\subsubsection{Motor Control}

The operator needs accurate control of the locomotion motors to successfully navigate HADES though an underground mine. The Electronic Speed Controllers (ESC) support four different control methods (Section 6.1); the operator needs to be able to switch between these depending on the terrain. As well as switching control methods, the operator needs to be able to make accurate low speed adjustments and travel at high speeds, and so a soft gearbox is implemented. When operating at low speed, the operator can select a lower gear and have a greater control precision. When operating at higher speeds and control accuracy is not needed, the operator can select a higher gear. As HADES can operate inverted, the control outputs can be inverted using a button on the X-box controller.

The control signal for the motors is received from the X-box controller (Section 7.1.3.1). The signal from the controller is noisy and highly sensitive to operator input. To smooth the operator input and reduce the noise, a moving average filter is implemented. The length of the filter is set to 10 which equates to a FIR low pass filter with a cut-off frequency of $7.5 \mathrm{~Hz}$ [20]. The joystick 
values range from - 1 to 1 , these are used to produce output values for each locomotion wheel. Equations 7.1 and 7.2 show equations for the left and right side wheels respectively.

$$
\begin{aligned}
& L_{s}=G *\left(A_{f}-A_{t}\right), \\
& R_{s}=G *\left(A_{f}+A_{t}\right),
\end{aligned}
$$

where $G$ is the selected gear box gain, $A_{f}$ is the amplitude of the forward motion and $A_{t}$ is the amplitude of the turning motion. As a safety function, a brake button should override the other motor control instructions, stopping the motors. When the brake button is pressed, the hades_locomotion node publishes the brake motor command. As discussed in Section 6.1 a break current of $15 \mathrm{~A}$ is needed to hold HADES stationary. To minimise the bandwidth utilisation of the communication link, the output signals are decimated from $200 \mathrm{~Hz}$ to a maximum sample rate of $10 \mathrm{~Hz}$. This limits the bandwidth utilisation for the motor_ctrl (9 bytes) msg to $90 \mathrm{Bps}$, while still maintaining good control of the robot.

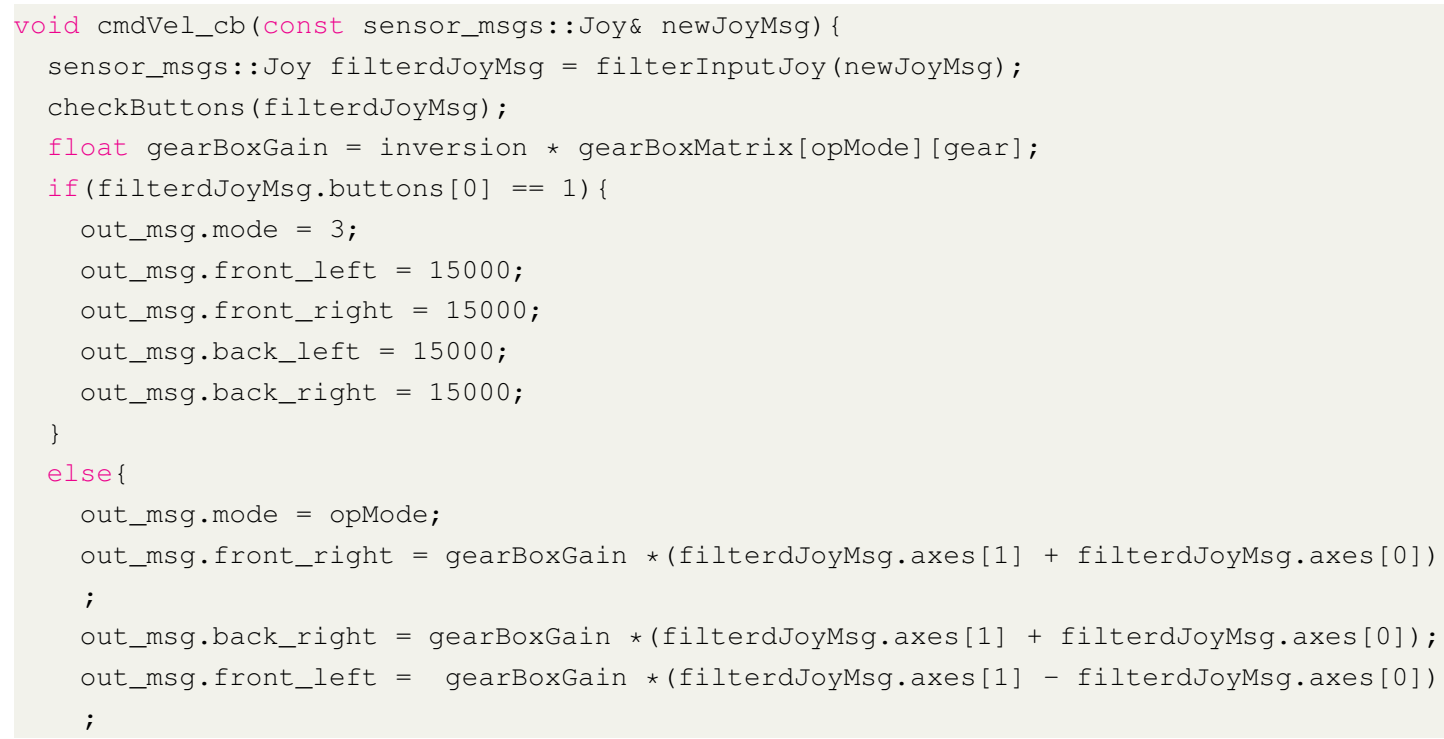




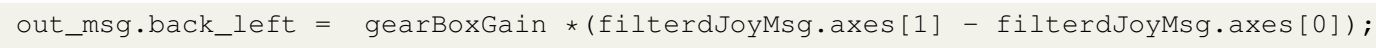

Listing 7.5: The ROS callback for an incoming joy message from the X-box controller.

Listing 7.5 shows the callback for the incoming message from the joystick. In this callback, the low-pass filter, kinematic equations and soft-gearbox code can be seen. The low pass filtration is handled by the filterInputJoy() function, which returns a filtered message. The code then looks for a positive edge on the inversion, operational mode and gear change buttons using the checkButtons() method. Based on the state of the buttons, the correct gearbox gain (gearBoxGain) is selected from a lookup table.

Using the gearbox gain and equations 6.1 and 6.3, the outputs for the motors are produced and stored in the out_msg. The out_msg is published in a separate loop running at $10 \mathrm{~Hz}$. Figure 7.4 shows the data flow diagram for the hades_locomotion node.

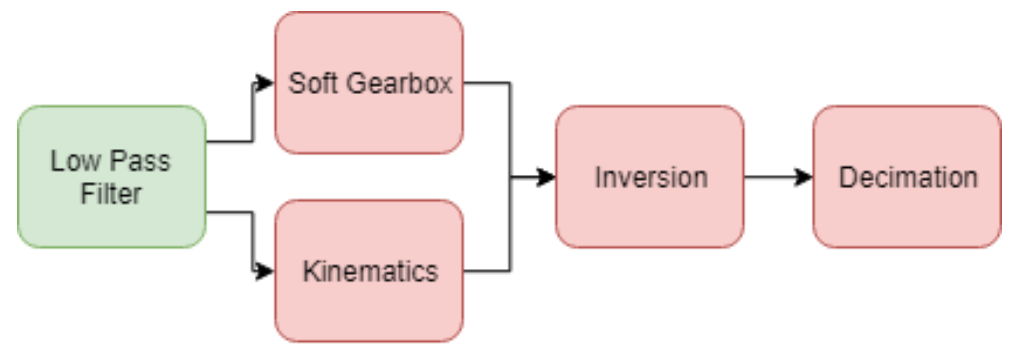

Figure 7.4: The data flow diagram for the hades_locomotion package

\subsubsection{X-box controller}

The operator needs a physical interface for controlling the HADES. The interface needs to allow the operator the accurately navigate HADES though com- 
plex terrain and provide sufficient buttons for common robot functionality. As it is possible that a person might operate HADES with minimal training, the physical interface should be intuitive and easy to learn.

The physical interface chosen is the Microsoft X-box controller. The X-box controller follows a common format for game controllers and has been proven to be intuitive for unfamiliar users $[44,14]$. The X-box controller has 12 buttons, two dual axis joysticks and two analogue triggers. Figure 7.5 shows the mapping of each control surface to the respective control parameter for the locomotion control.

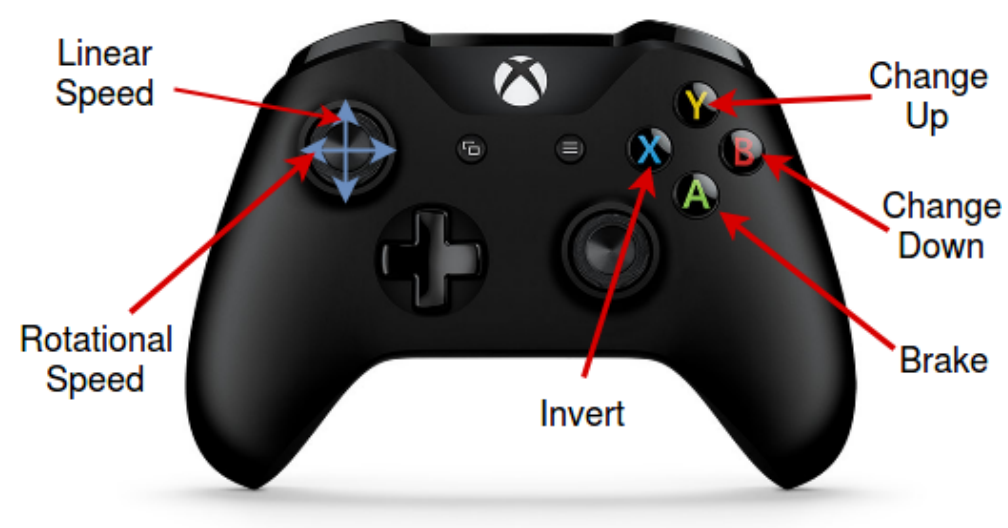

Figure 7.5: The mapping of the different control interfaces to each locomotion control.

To interface with the X-box controller, the ROS joy_node is used. The joy node contains the drivers for the controller and produces a joy message. To prevent vibration of the controller from triggering movement, the joy_node is configured to increase the dead-zone of the joysticks. The joy_node also decimates the joystick signal to $100 \mathrm{~Hz}$, to allow the low pass filter to function accurately. 


\subsubsection{Summary}

The ROS control system running on the NUC provides overarching control of HADES and the interface to the operator. Each ROS node performs a key robot function and allows HADES to be controlled by an unskilled operator. A significant error checking system was implemented, that reports and attempts to remedy faults in the NUC and hardware system. To allow a video stream to be used for teleoperation, a bespoke video multiplexer was developed. The bandwidth of the video for the communication system was reduced by the implementation of the Theora image compression algorithm. A locomotion control node is used to receive inputs from an X-box controller and produce control outputs for the drive system. The locomotion control implements a soft-gearbox that allows the operator to move slowly and precisely or at higher speeds. The ROS control system allows an operator to navigate HADES through an underground mine environment intuitively with access all of HADES' sensor systems.

\subsection{Graphical User Interface}

The operator needs to view the camera, and sensor data feeds from the robot, as well as access a configuration utility for setting robot parameters. To achieve this a Graphical User Interface (GUI) is required. The GUI should allow the operator to view the video and telemetry streams from the robot and reconfigure the robot parameters. It also needs to be intuitive and easy to use for a nonexperienced operator. The GUI was developed as an extension of the original thesis goals. Thus, it is only an exploration into the potential form and function and is not completed and as detailed in Section 10.2, is an interesting project itself.

To implement the prototype GUI, the QT graphical user interface library is used. The QT library allows for rapid prototyping of complex GUI systems as 
it contains most of the functionality needed. If the functionality is not included in the base library, a large number of user-contributed widgets are available. To integrate into the ROS environment the qt_ros package is used. The qt_ros provides functionality for connecting to the ROS master and parameter servers, as well as generating a boilerplate program to expand on.

The prototype GUI interface is split into four sub classes, the connection_window class, the main_window class, the configuration_window class and the qnode class. The connection_window class maintains the connection to the ROS servers and presents the real time log information from the running ROS nodes. Figure 7.6 shows the GUI presented by the connection_window class.

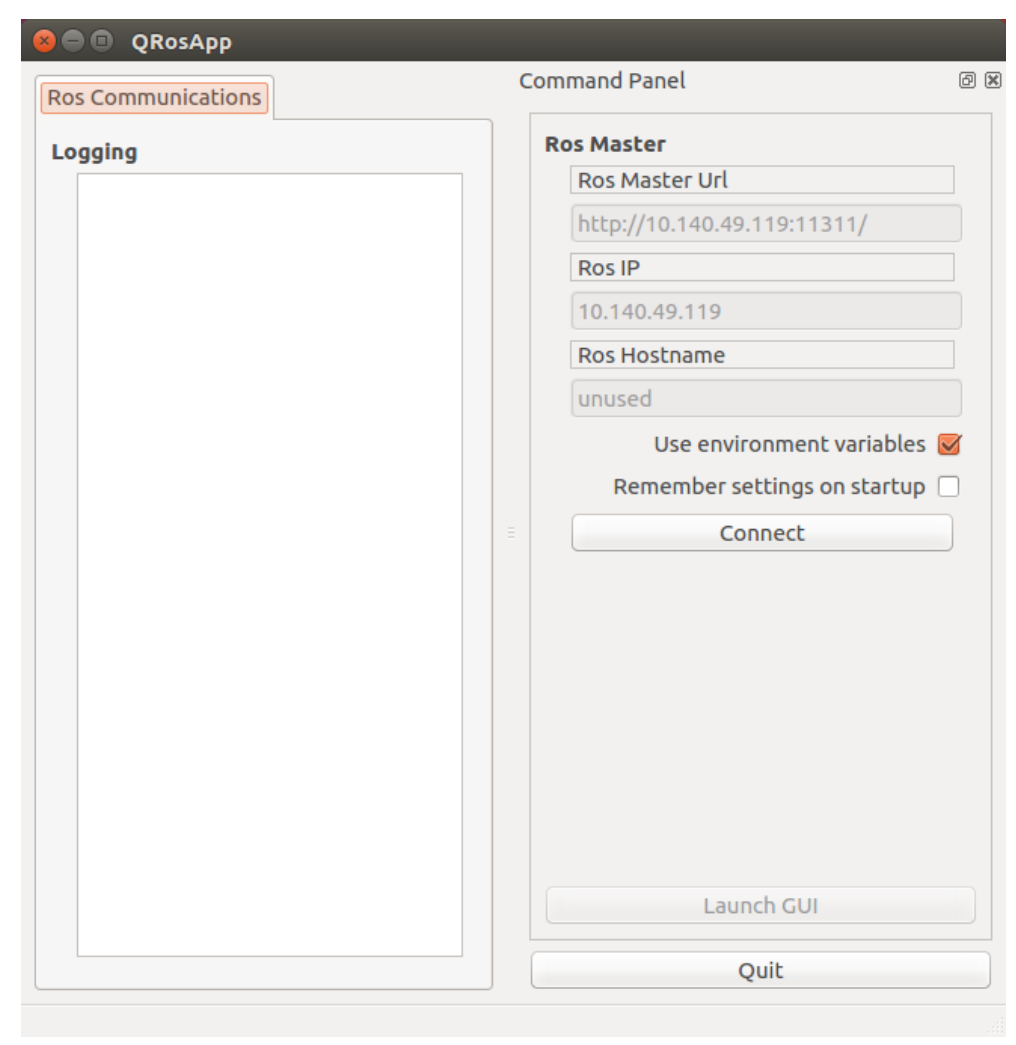

Figure 7.6: The GUI presented by the connection_window allowing the operator to connect to the ROS servers and view the real-time log information. 
The GUI allows the operator to specify the IP address of the ROS master and the IP address of the current node. When the user clicks the connect button, the GUI creates an instance of the qnode class and passes the user-entered parameters. The qnode class handles all the back end communication between ROS and QT. On creation, the qnode will try and contact the ROS master server; if it can do so, it registers all the GUI's subscribers and publishers. Once the registration is complete the main_window class can be launched with the Launch GUI button.

To pass data between classes, the QT environment does not use messages and topics like the ROS environment, instead signals and slots are used. A signal is a data type that is emitted by a class, the signal is then received by another class in a slot. Signals and slots are used to pass signals between the front and back ends of the GUI, the main_window class and qnode class respectively. The qnode converts the signals to/from ROS messages for the front end. Listing 7.6 shows the function for receiving the motor_data message from HADES and emitting a signal for the main_window class.

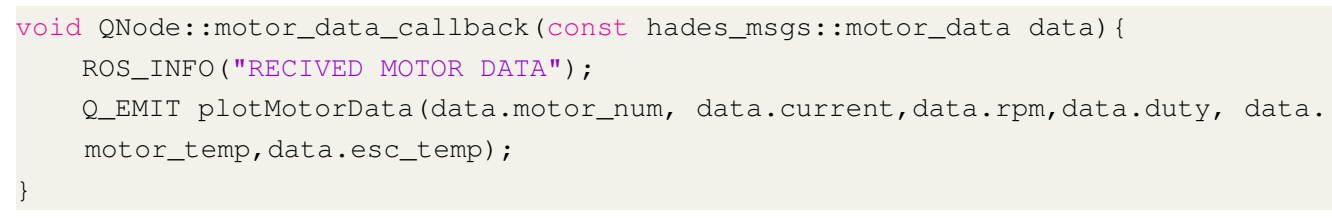

Listing 7.6: The ROS callback for the motor_data message and the function for emitting the data to the main_window class.

Listing 7.6 is the callback for the motor_data received event. In this callback the message is unpacked and emitted to the main_window class using the $Q$ EMIT macro.

The main_window class is the main operating window for HADES, the GUI for this can be seen in Figure. 7.7. The video stream of HADES' cameras is critical for navigating the robot. For this reason, it is permanently displayed on 
the left half of the GUI. Below the video stream, there are drop down boxes for selecting the camera feed and the video compression settings.

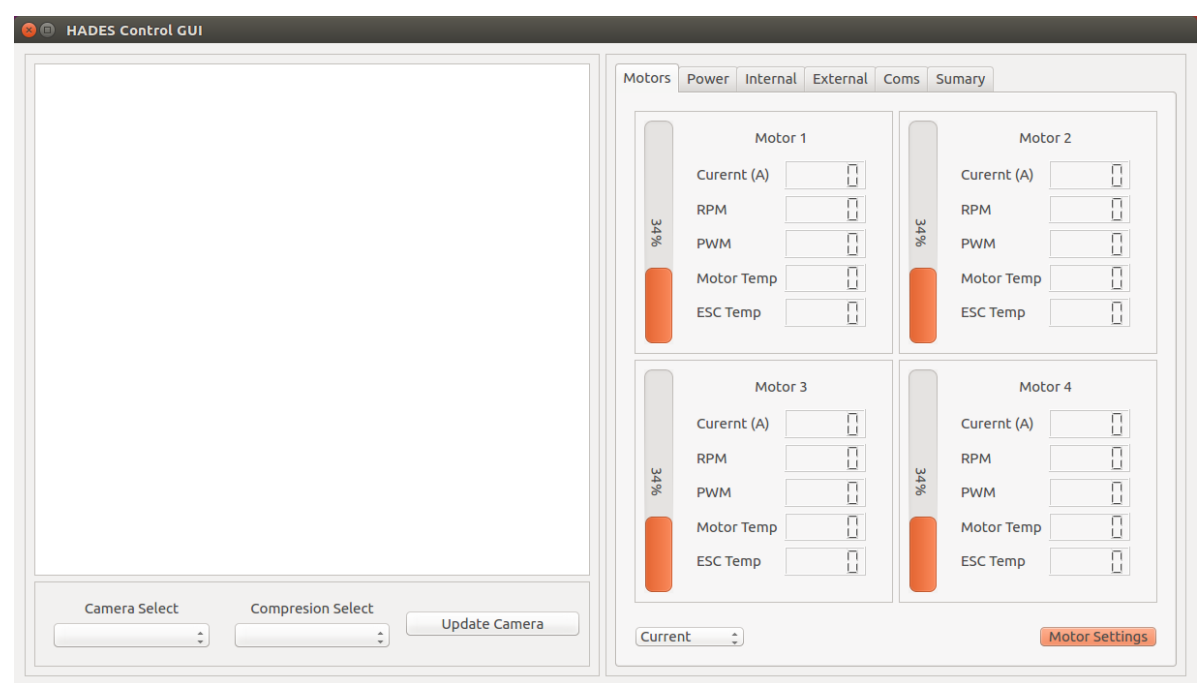

Figure 7.7: The GUI interface for the main window class.

The right side of the GUI displays the robot telemetry. Six tabs are used to divide the information, one for each HADES' functional systems. The display tab for the motor information can be seen in Fig. 7.7. To allow the operator to quickly assess the operating conditions of the motors, one aspect of the motor data is displayed on the bar-graphs. For a more in-depth assessment, the remaining data is displayed on digital readouts beside the bar-graph. The operator can select the data stream they want to display on the bar graphs with a drop-down box.

To configure the less frequently accessed parameters of HADES, the configuration_window class is used. Figure 7.8 shows the GUI presented by the configuration_window class for the motor settings.

The GUI is designed so that multiple instances of it can be run in the same ROS network which allows for multiple operators to manage different aspects of the robot if necessary. The GUI will update across all instances to reflect the 


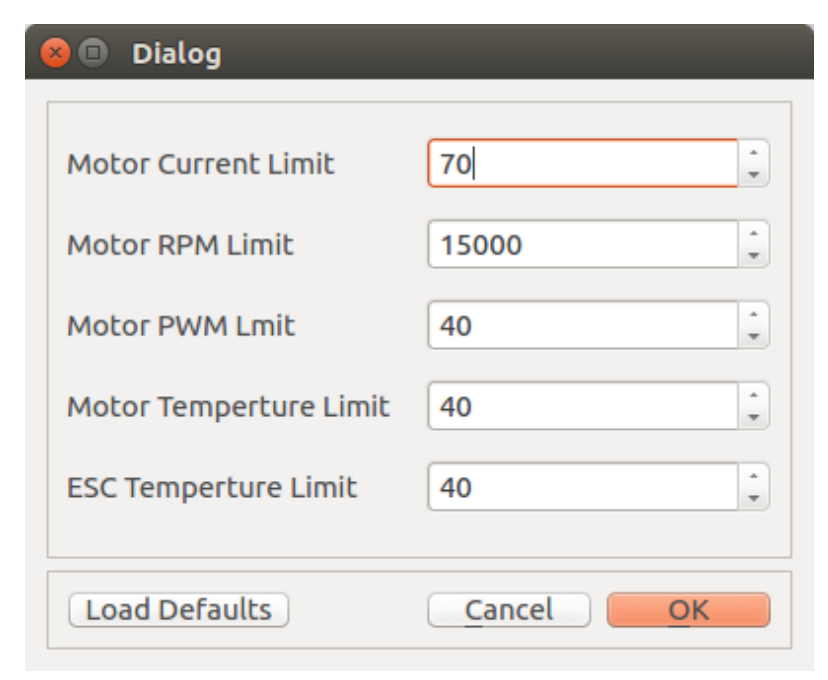

Figure 7.8: The configuration settings for updating the motor drive settings.

change that one operator makes.

\subsubsection{Summary}

The prototype GUI has started the exploration into the user interface that will allow an unskilled operator to successfully and safely navigate HADES though an underground mine. By defining different levels of operation interaction, the GUI can remain simple for an unskilled operator, but a skilled operator can still access to the advanced settings if necessary. The QT user interface library has been integrated into the ROS environment, and several prototype display windows have been implemented. The GUI was developed to enable initial testing of HADES, subsequent development to expand the GUI to all areas of robot function is needed. The continued development must include a user study to gauge the usability of the GUI. Due to time constraints, and as a user study requires ethics approval, it is considered out of the scope of this project. 


\section{Chapter 8}

\section{Embedded Software}

The sensors and actuators in HADES are all controlled with microcontrollers. Each microcontroller is designed to function as an independent sub-system, while executing an independent process. To complete the tasking of each of the sub-systems, four of the microcontrollers require bespoke embedded software. The embedded software design needs to ensure that the sub-systems remain independent and can reliably complete their tasking. The embedded software must also support complex error checking and redundancy of critical systems.

Figure 8.1 shows the HADES hardware system diagram. Each of the microcontrollers with bespoke software (except for the watchdog controller) use the Arduino $\mathrm{C}++$ build and runtime environment. The Arduino build system increases prototyping speed as there are many pre-written libraries for interfacing microcontroller and external peripherals.

The embedded code is spread between nine microcontrollers. To ensure all the sub-systems function cohesively, a system-wide state machine is implemented. The state machine enables each of the subsystems to be aware of the current function mode of the robot - including the state of the sibling microcontrollers. This ensures coherency of command exchange and communication be- 


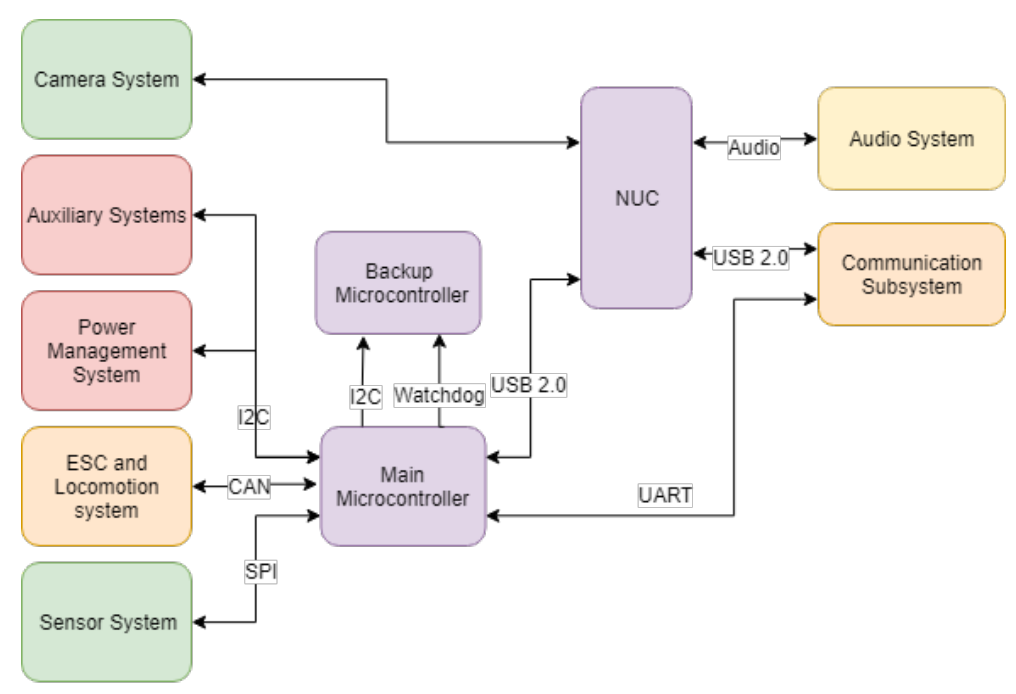

Figure 8.1: The communication diagram for the hardware system in HADES.

tween the microcontrollers. The system-wide state diagram is shown in Fig 8.2.

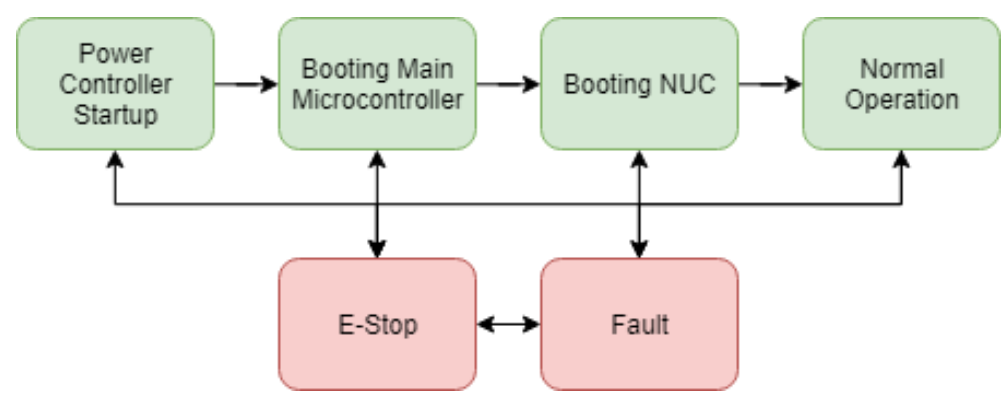

Figure 8.2: The state diagram for the embedded software system.

During startup the power controller (Section 8.3) governs the start up states. The sates insure that the system does not try to implement a process that uses an unpowered system component. After start up, the main microcontroller assumes control of the system and provides an interface to the operator though the NUC and communication system. At any point during start up, or normal operation the system can enter a fault or emergency stop condition. A fault is a condition that is not going to damage the robot, or surrounding personnel (i.e. I2C coms bad checksum). An emergency stop is a condition where the robot 
needs to be put immediately into a safe state.

This chapter covers the design and implementation of the bespoke embedded software that runs on the power management controller, the main microcontroller, the auxiliary systems controller and the watchdog controller.

\subsection{Low level Communication}

As discussed in the overview, the embedded code is split between four microcontrollers; the main controller, power controller, the auxiliary system controller and the watchdog controller. Each of these controllers communicate over I2C and two shared digital IO lines. The I2C standard supports the physical and data layers of a protocol stack, therefore only the application layer needs to be developed. The application layer needs to describe a common command set and the packet structure for each command. As the I2C standard does not inherently have error checking, the application layer will need to integrate a bespoke error checking scheme.

I2C uses two basic message types for communicating between the master and the slaves; a data message and a request message. The data messages are used for passing data whereas the request message is for when the master requests information from the slave. The bespoke application layer describes the generic data message format, shown in Table 8.1, where the maximum length of an I2C packet is 32 bytes. To ensure the data received is accurate, a checksum generated with the CRC-8 algorithm is appended to every data message.

Table 8.1: The standard packet for communication on the I2C bus.

$$
\begin{array}{|l|l|l|}
\hline \text { CMD (1 byte) } & \text { DATA(30 bytes max }) & \text { CRC (1 byte }) \\
\hline
\end{array}
$$

The CRC-8 algorithm (Cyclic Redundancy Check) generates an 8-bit checksum by dividing the polynomials of the data array [41]. The type and size of 
polynomial division depends on the size of the data array and the type of CRC8 algorithm used. As completing the polynomial division for every data array is computationally expensive, a lookup table is used. The lookup table is generated using the Dow CRC-8 algorithm [41, 69]. Both the sender and the receiver calculate the CRC for the packet. The two CRC values are compared and if they are different, the message is considered invalid and a new message is requested.

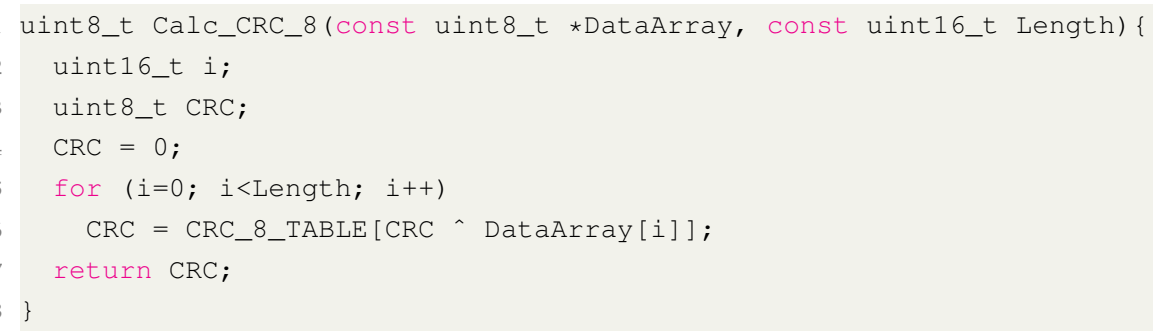

Listing 8.1: The CRC-8 calculation method using the lookup table

Listing 8.1 shows the function for calculating the CRC for DataArray of length (length) using the lookup table CRC_8_TABLE. The CRC is initially set to 0 and the index of the first CRC code in the lookup table is generated by CRC XOR DataArray[0]. The first CRC code is then used in the same manner to produce the remaining CRC codes for each position of the data array until a final CRC is reached. This operation can be expressed mathematically by Eqn. 8.1.

$$
C R C=T[T[T[0 \oplus D[1]] \oplus D[2]] \ldots \oplus D[N-1]]
$$

where $C R C$ is the final CRC, $T[i]$ is the CRC lookup table at position $i$ and $D[]$ is the data array of length 0 to $N-1$.

The two message types are processed on a slave with two interrupt driven methods receivedEvent() and requestEvent(). The receivedEvent() method handles the processing of an incoming data packet. A switch-case statement is used to determine the correct action based on the command byte of the packet. List- 
ing 8.2 shows an abbreviated version of the receivedEvent() method, highlighting the process for setting the BMS relays on the power PCB (Section 6.2.2).

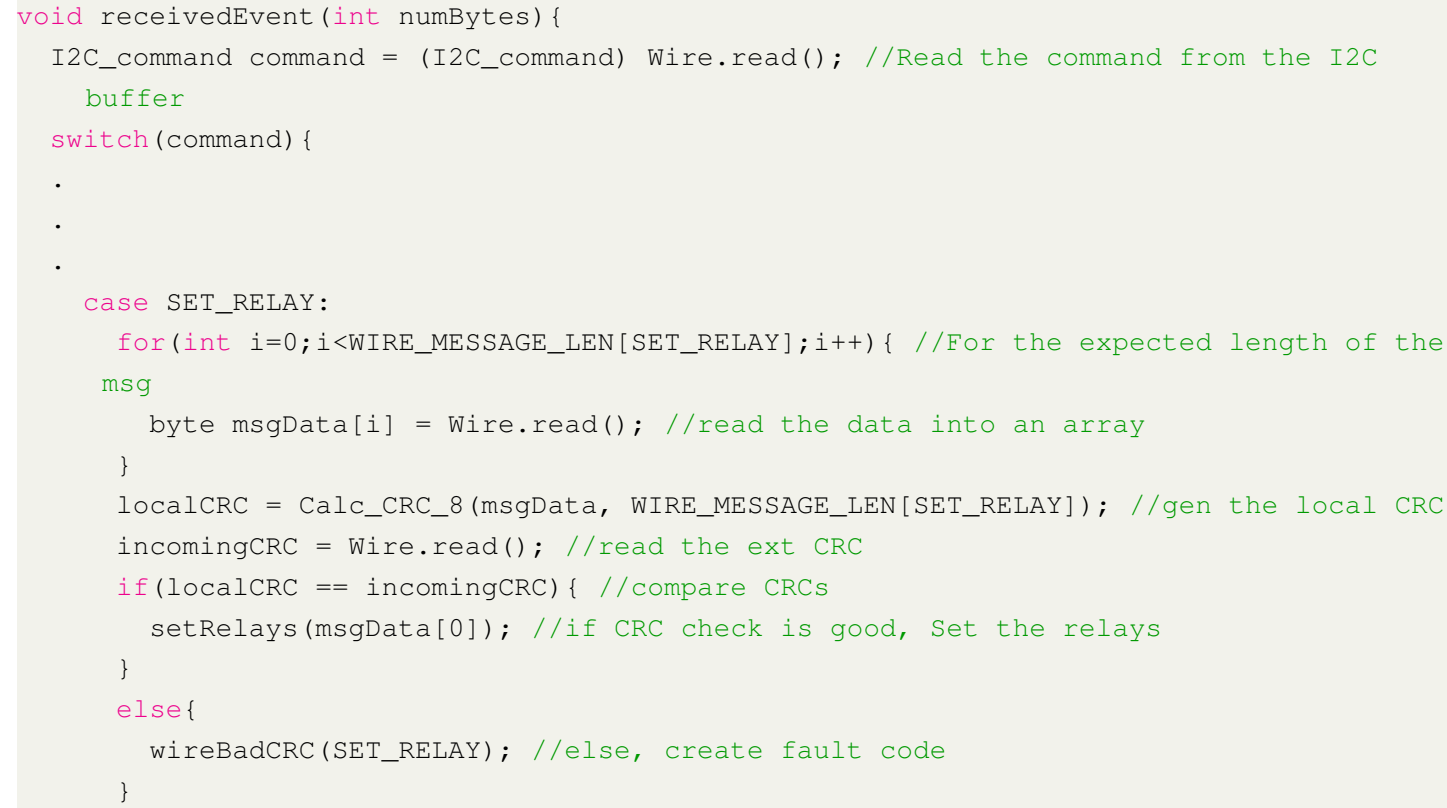

Listing 8.2: A snippet of the receivedEvent() method, highlighting the process for setting the BMS relays

Listing 8.2 is the start of the receivedEvent method, used for receiving data on the power controller. The code starts by reading the command byte from the I2C buffer using the Arduino Wire library. The command byte is parsed to an I2C_command enumerator for use in the switch case statement. The received command is used to determine the length of the data stored in the I2C buffer which is then read into the data array msgData. The local CRC value is then generated from the data array. If the local and received CRC values match, the data is processed. If the values do not match, a fault code is generated and a new message is requested. 
As the Wire.read() method returns an unsigned char, complex data types have to be constructed with an array of datatype unsigned char. This is done in code with a union; where two variables share the same memory address. Listing 8.3 shows the union used for converting an unsigned char array to a bat_data structure, a structure used to store the voltages, current and temperature of a system battery.

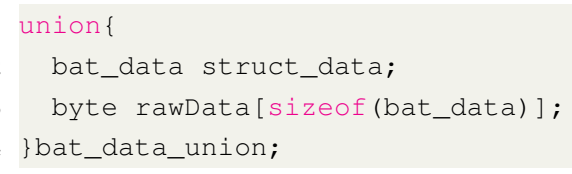

Listing 8.3: The data union between the bat_data struct and an unsigned char array.

The two digital lines shared between all the hardware sub-systems are used to communicate fault and emergency stop conditions quickly. The two lines are connected to bi-directional IO pins on every microcontroller. During normal operation, the IO lines are pulled high by all the microcontrollers. When a microcontroller generates a fault or emergency stop condition, the IO line is pulled low. The IO lines are serviced by Interrupt Service Routines (ISR) on each microcontroller. Listing 8.4 shows the code for fault line on the main microcontroller.

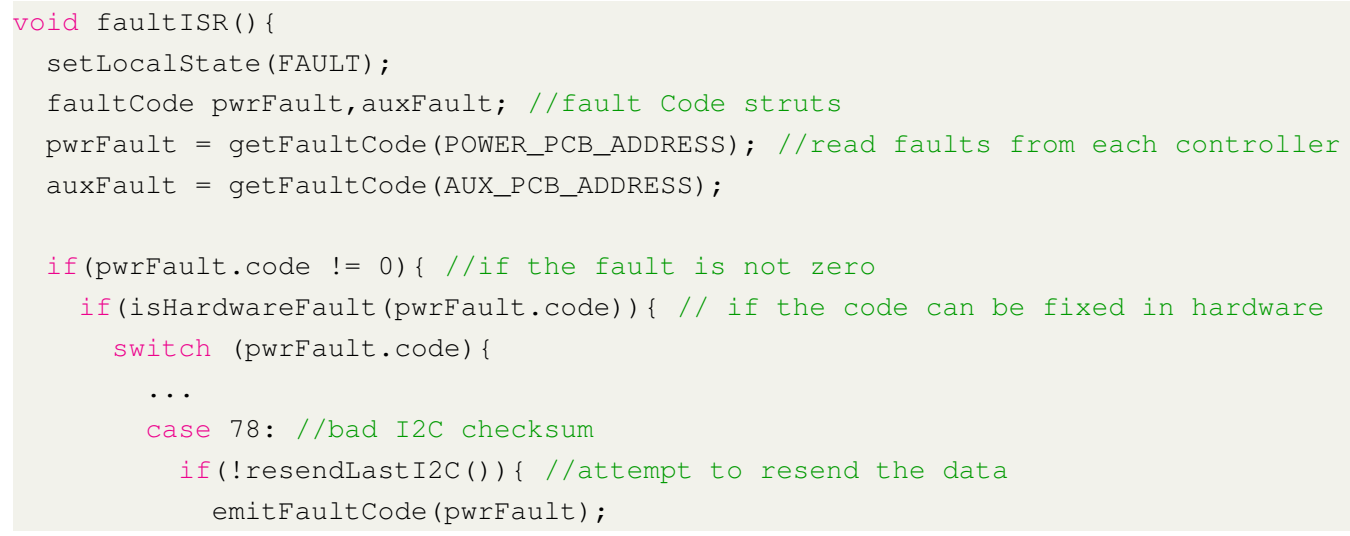




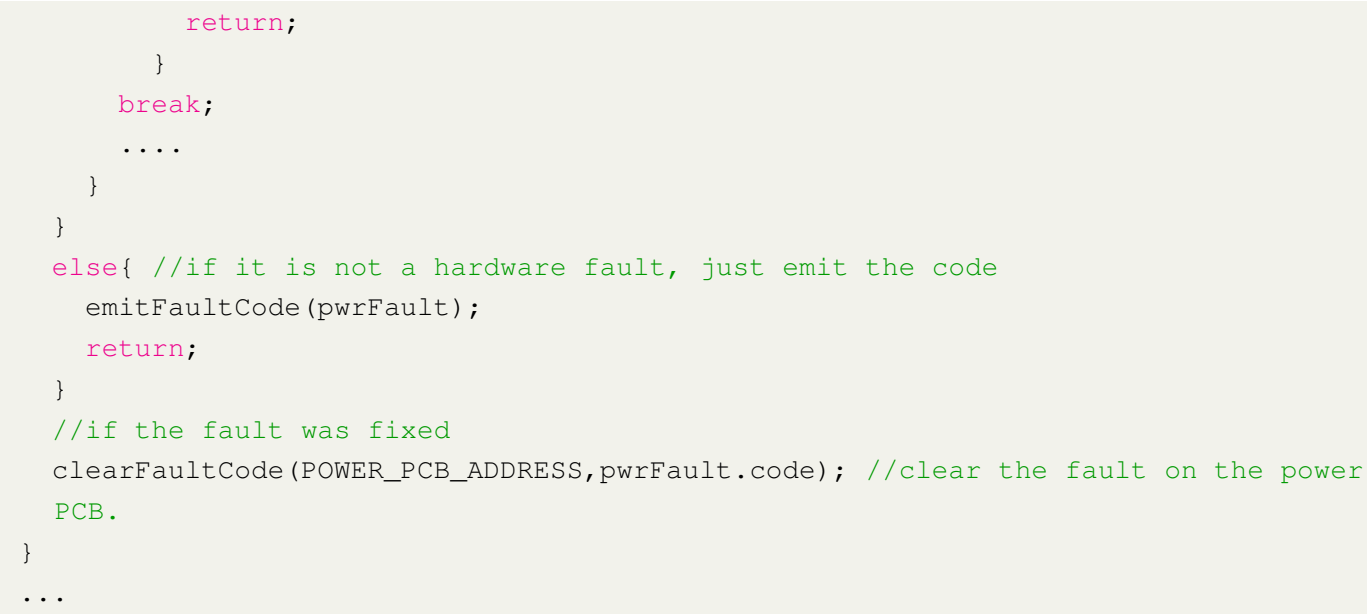

Listing 8.4: A snippet of the ISR that services the fault line in the main microcontroller.

In the ISR for the fault line on the main microcontroller, the fault code is first requested from each of the slaves. The format of the fault codes is extended from the fault code system used in the ROS control system (Section 7.1.1), where a fault code is represented by three bytes of information. The fault code system is designed so that fault codes between 127 and 192 can be handled at the hardware level by the main microcontroller. If the fault is remedied by the main microcontroller, the fault code is cleared on the slave microcontroller. If the fault is unable to be fixed and the NUC is running, the fault code is published to ROS, otherwise it is sent to the communication system over the back-up serial link.

\subsection{Main Microcontroller}

The main microcontroller is the hub of the hardware system and the link to ROS on-board the NUC. In usual operation, it coordinates the operations of the other controllers and gathers environmental data from sensors internal and ex- 
ternal to the chassis. If the NUC fails or is shut down, the main microcontroller becomes the highest level controller in the HADES system.

The main microcontroller uses Zilch (an Arduino library) to schedule and manage different tasks. Using Zilch allows for multiple threads to be run simultaneously allowing different tasks to be paused and resumed without affecting the total function of the controller. As the threads can require access to key software/hardware resources simultaneously, the use of these resources must be carefully managed. As an example, listing 8.7 shows the resource checking for the SPI bus where if two threads attempt to access the SPI bus simultaneously, the data will become corrupted.

\subsubsection{ROS Integration}

The main microcontroller is the link between the hardware system and the ROS controller. The main microcontroller is connected to the NUC with a USB 2.0 link. To allow the TCP/IP ROS messages to pass over the USB link, a ROS serial node is used. The serial node serialises and deserialises the ROS TCP/IP data for transmission over the USB link. Upon start-up, the main microcontroller needs to register with the serial node to ensure the correct topics are transmitted.

The main microcontroller subscribes and publishes messages on 15 different topics. Table 8.2 shows the received and published message types from/to the main microcontroller. To subscribe to the data coming from ROS to the microcontroller, a call-back system is used. When data is received, the code triggers an interrupt that services the new data meaning that the serial port does not have to be continually polled. Listing 8.5 shows the start of the ROS call back for the motor control data. In the callback, the motor_ctrl is received from the serial node. Based on the expected command mode in the message, the drive motors are set using instances of the VESC_CAN class (Section 8.2.2.1). To min- 
Table 8.2: The ROS messages subscribed and published by the main microcontroller

\begin{tabular}{|l|l|}
\hline Subsribed & Published \\
\hline sensor_ctrl.msg & sensor_data_int.msg \\
\hline bms_settings.msg & sensor_data_ext.msg \\
\hline rail_settings.msg & bat_data.msg \\
\hline motor_setting.msg & rail_data.msg \\
\hline motor_ctrl.msg & error_data.msg \\
\hline thread_settings.msg & imu_data.msg \\
\hline led_ctrl.msg & odom_data.msg \\
\hline coms_ctrl.msg & \\
\hline
\end{tabular}

imise bandwidth, the motor_ctrl uses 16-bit integers to store the control data. The control data can be used to represent a 32-bit float for current and duty cycle control or a 16-bit integer for RPM control.

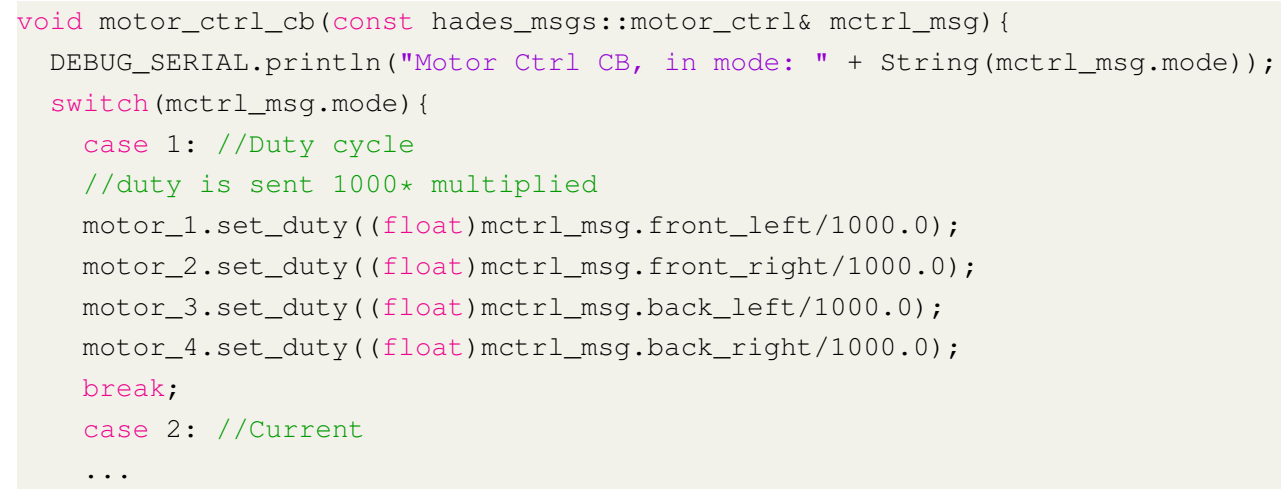

Listing 8.5: The ROS callback for the motor control data.

To publish data from the micro-controller to ROS, specific Zilch threads are used for each topic. By allotting a thread for each publisher, the bandwidth utilisation of the USB link can be minimised. Depending on the data type, dif- 
ferent publishing rates are used and can be re-configured during operation with a rate_ctrl message. Listing 8.6 shows the Zilch thread for publishing the output data from the IMU filters.

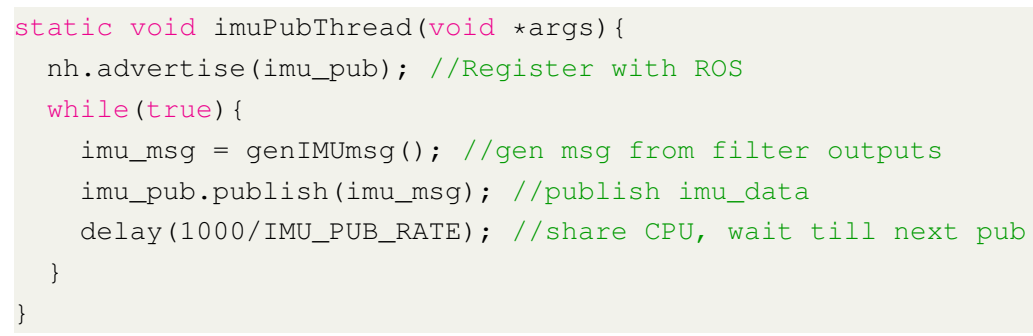

Listing 8.6: The scheduling thread that generates and publishes the IMU data message.

The thread initialises the ROS IMU publisher with the serial node and enters normal operation. In normal operation, the imu_msg is generated by the genIMUmsg() function, and it is published. Zilch will context switch to a different thread when a delay() or yield() is called. The delay in milliseconds for the IMU thread is calculated from the global variable IMU PUB RATE.

\subsubsection{Inertial Measurement Unit and Sensor Fusion}

Two MPU9250 nine-axis Inertial Measurement Units (IMU) are used in HADES to monitor the pose and translational movement of the rear arm and main chassis. As discussed in Section 5.2.3.1, to accurately track the pose and prevent aliasing from the vibration of the Whegs, the IMU needs to be sampled at a rate greater than $100 \mathrm{~Hz}$. The raw data from the IMU needs to be passed to a sensor fusion algorithm to remove noise and increase accuracy.

The IMUs are connected to the main microcontroller with SPI; this means they can be sampled at a maximum rate of $10 \mathrm{kHz}$. During initial testing, a 
sample rate of $150 \mathrm{~Hz}$ was found to provide a good compromise between data accuracy and CPU utilisation. A thread of the Zilch scheduling operating system was used to maintain the sample rate at $150 \mathrm{~Hz}$.

To track the pose of the robot, three different algorithms were investigated: the Kalman Filter, the Madgwick algorithm and the Mahony algorithm [47,61]. A Kalman filter is a generic sensor fusion algorithm while the Madgwick and Mahony algorithms are dedicated pose estimation algorithms. The Kalman and Madgwick filters are both 9-axis (or more) filters, while the Mahony filter is only 6-axis. This means the Mahony filter cannot include the magnetometer reading in the pose estimation. The Kalman filter has been shown to be significantly less efficient than the Madgwick algorithm for 9-axis sensor fusion, thus, the Madgwick algorithm was chosen [47].

The Madgwick algorithm is an optimised gradient descent algorithm that uses Euler angles to represent the pose. The Madgwick algorithm is already implemented as an Arduino class and two instances of this class are used to track the pose of the rear arm and the main chassis respectively. The pose filters are updated with the new IMU data at the original IMU sample rate $(150 \mathrm{~Hz})$.

The translational displacement of the chassis is tracked with a Kalman filter. The Kalman filter combines the pitch/yaw/roll data from the Madgwick algorithm and a double integration of the raw accelerometer data. The double integration of the acceleration data produces a noisy signal that is prone to drift. Thus, the translational estimation is likely to become increasingly inaccurate, however, as the robot is tele-operated the translational data only assists the operator, it is not mission critical.

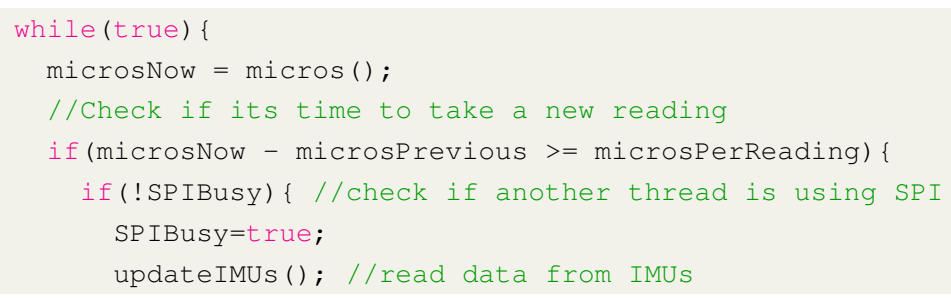




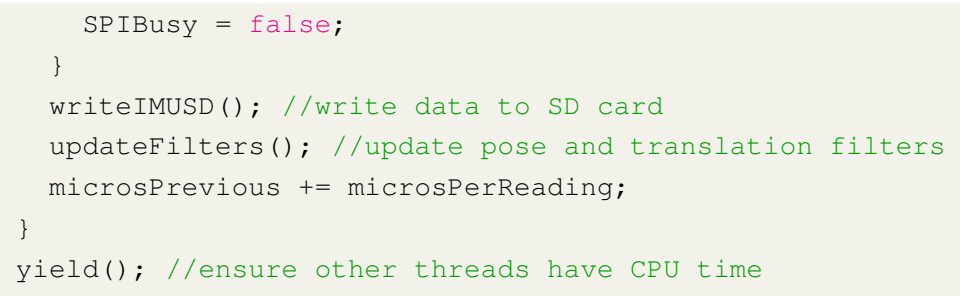

Listing 8.7: A snippet of the scheduling thread that reads the IMU and updates the filter values.

Listing 8.7 shows the Zilch thread used for reading the IMUs and updating the filters. The thread runs as an infinite loop, using the micros() function to trigger when a sample is taken. The IMUs are connected to the main microcontroller with SPI (Section 5.2.3), the global variable SPIBusy is used to prevent multiple threads from accessing the SPI bus. The updateIMUs() function reads the data from the IMUs and stores it in local memory. The updateFilters() method then updates the pose and translation filters from the local memory. To ensure that no data is lost, the raw IMU data is saved to the hardware system $\mathrm{SD}$ card at the original sample rate with writeIMUSD() method. To minimise the bandwidth use of the USB link while still providing a useful data stream, the pose and translation data is published to ROS at a default rate of $5 \mathrm{~Hz}$, using a separate thread.

\subsubsection{Motor Control}

Four Vedder ESCs (VESCs) are used to control each of the locomotion motors and are connected to the main microcontroller with a CAN-Bus interface (Section 6.1). To control and request data from the VESCs over the CAN-Bus, a software interface is needed. The VESCs follow a predefined API for communicating and so the software interface needs to conform to this API. The software interface also needs to allow for communication to multiple VESCs. 
When used without encoders, the VESC API supports four modes of control: duty cycle, motor current, RPM and brake current. Duty cycle control is open loop control, the remaining three options are closed-loop control with each VESC running an independent control system. When used in closed-loop control, the VESC alters the duty cycle of the drive transistors to try and meet the target RPM/current. As defined in Section 7.1.3 the operator can switch between different operational modes depending on the terrain.

The VESC monitors the conditions of the motor, battery and drive transistors. If any of the readings exceed pre-defined safe working thresholds, the VESC will implement a backoff strategy where motor power is reduced until safe working conditions are achieved. The measured values, as well as any soft-back percentage, can be requested from the VESC.

An Arduino class simplifies CAN communication to the VESCs by combining all the necessary functions and properties into a single object. A VESC_CAN object is created for each motor and assigned an ID relative to its position on the robot: front left (1), front right (2), back left (3) and back right(4). This allows independent initialisation and control of the motors, reducing the risk of a single fault affecting the complete drive system. The header file for the VESC_CAN class can be seen in Listing 8.8.

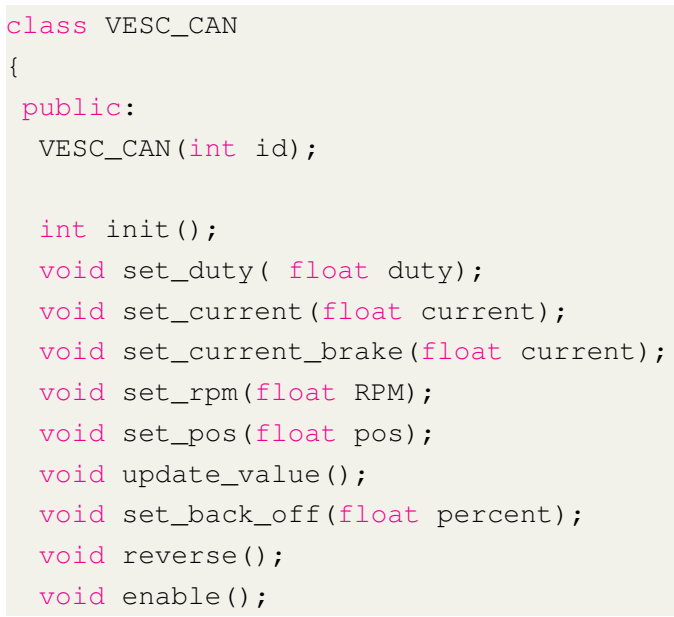




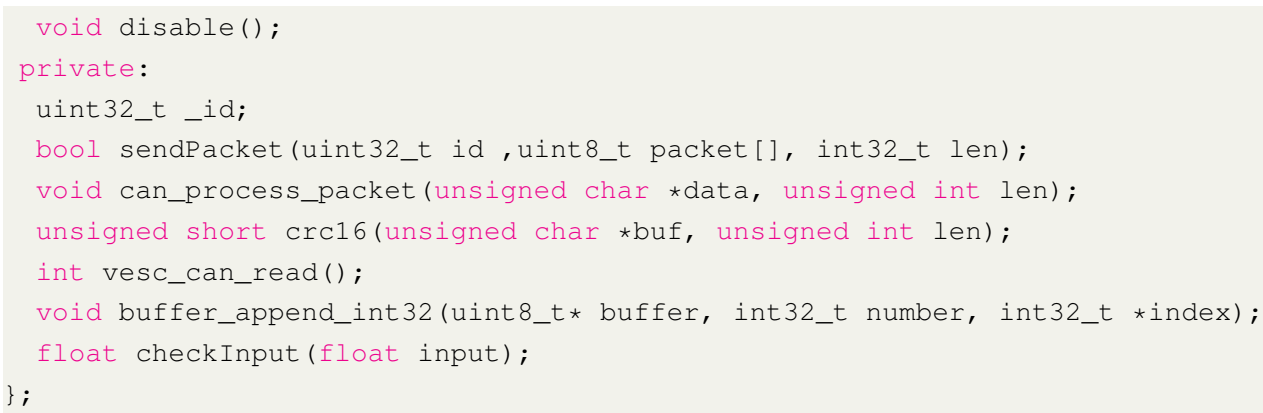

Listing 8.8: A snippet of the header file for the VESC CAN class.

The VESC uses the extended CAN packet for control and data retrieval. An extended CAN packet increases the payload from 48 bytes to 64 bytes, it also extends the address variable size from 11 bits to 29 bits [66]. The larger address size is needed as the VESC uses the address bits for the address and the command. The command is an enumerated data type that dictates the action the VESC will take (i.e. constant RPM control). The address variable is packaged so that the command is the upper 20 bits and the address is the lower 9 bits.

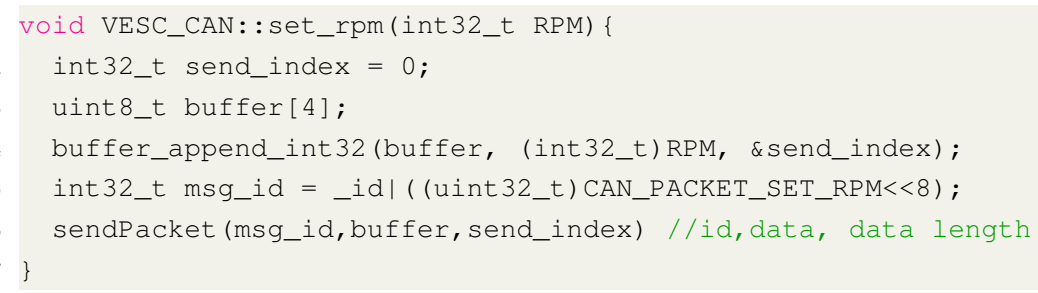

Listing 8.9: The set RPM method for controlling the target RPM of a VESC.

Listing 8.9 shows the function for setting the target RPM of the VESC. In this function the 32-bit integer is packaged into the 4-byte data array (buffer) with the buffer_append_int32 function. The id (_id) of the target VESC is bit-shifted and bit-wise OR'd with the set RPM command (CAN_PACKET_SET_RPM) to create the message ID (msgid) variable. The packet is then sent using the sendPacket() function. 
In the case of a single VESC implementing a soft-back off, the other motors in the system need to compensate to maintain controllability and straight line travel. This is completed by reducing the output power of the other three motors to match the reduced VESC. If more than one VESC is running reduced power, the VESC with the lowest power is used to set the output power of the other VESCs.

The VESC maintains a counter to track the number of commutation cycles completed which can be used to produce wheel odomentry. The distance $(d)$ travelled by each wheel can be given by,

$$
d=\frac{C / M_{p}}{G_{r}} \cdot 2 \pi r
$$

where $C$ is the number of commutation cycles completed, $M_{p}$ is the number of motor polls, $G_{r}$ is the gearbox reduction and $r$ is the radius of the wheel. The Hobbystar 56112 motors have 8 polls and are used with a 64:1 gearbox (Section 4.2), the Whegs (Section 4.1) have a radius of $245 \mathrm{~mm}$. The odometry data is published to ROS at a default rate of $10 \mathrm{~Hz}$. The wheel odometry data is likely to be inaccurate as HADES relies on skid steering for changing directions. To increase the accuracy and reliability of the odometry, sensor fusion could be used to combine the IMU readings with the wheel odomentry.

The commutation counts and other key data points are requested from each VESC at a rate of $50 \mathrm{~Hz}$ per VESC. Each request is timed so that the packets will not collide on the CAN-Bus network. The data is sent from the VESC in a predefined structure shown in Listing 8.10.

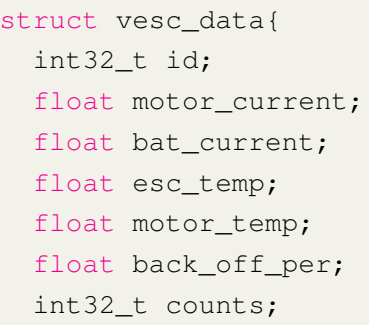


; ;

Listing 8.10: The data strucutre that is requested from the VESC.

\subsubsection{External and Internal Sensors}

HADES carries a comprehensive set of environmental sensors both internally and externally. These sensors are used to ascertain the condition of the mine and provide telemetry data to the operator. All of the environmental sensors on HADES produce analogue outputs that are sampled by ADCs. The sampled ADC readings need to be converted into usable metrics. The pressure sensors that are used for the positive pressure explosion protection system are triply redundant, to acquire data from these a sensor voting system is needed.

The code for the sensors is split into two Arduino classes InternalSensors and ExternalSensors for the internal and external sensors respectively. Each of the classes contains the functions and headers needed for managing and acquiring data from the sensor systems. Listing 8.11 shows the header file for public methods of the InternalSensors class.

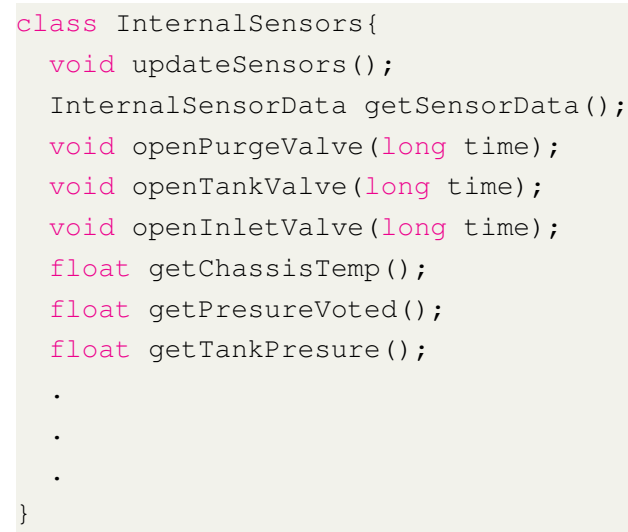

Listing 8.11: The start of the InternalSensors class declaration. 
The Alphasense A4 series sensors used to monitor the concentration of $\mathrm{CO}$, $\mathrm{H}_{2} \mathrm{~S}$ and $\mathrm{SO}_{2}$ around the chassis are temperature dependent sensors. To offset this temperature dependence, the sensors have two electrodes; the working electrode and auxiliary electrode. The working electrode responds to gas concentration and temperature change, while the auxiliary electrode only responds to temperature change. This means the auxiliary electrode can be used to off-set null the working electrode. The relationship between the ADC voltage inputs ( $V_{\text {Working }}$ and $\left.V_{\text {Auxiliary }}\right)$, and the gas concentration $\left(G_{c}\right)$ is given by,

$$
G_{c}=s\left(\frac{V_{\text {Working }}}{G}-n_{t} \frac{V_{\text {Auxiliary }}}{G}\right)
$$

where $s$ is the sensitivity of the sensor and $G$ is the gain of the transimpedance amplifiers. $n_{t}$ is a scale factor that is sensor and temperature dependent, the values of $n_{t}$ for different temperatures can be found in the sensor datasheet.

The City Technology ME2-O2 and IRcell-CO2 both have linear voltage to gas concentration outputs. The relationships between the ADC voltage and gas concentration are shown in rows one and two of Table 8.3 respectively. The City Technology 4P-50 explosive gas pellistor measures a number of different explosive gasses, so it cannot provide concentrations of independent gasses. The 4P-50 indicates how explosive the atmosphere surrounding the sensor is, expressed as a percentage of the Lower Explosive Limit (LEL). The relationship between the ADC voltage and the sensor metric can be seen in row three of Table 8.3.

The temperature, pressure and humidity sensors also have linear relationships between the ADC voltage and the sensor metric. These relationships can be seen in rows 4 - 6 of Table 8.3. Many of the gas sensors require calibration for correct operation, however, as many of the gasses to be measured are toxic, correct calibration is considered outside of the scope of the project. Calibration is normally performed by an approved contractor, they will measure the sensors response to know concentrations of it's target gas and adjust the constants of the curve fit equations to fit. 
Table 8.3: The relationships between the ADC voltages and the sensor target metric

\begin{tabular}{|l|l|}
\hline Sensor & Equation \\
\hline Oxygen - ME2-02 & $\%_{O_{2}}=100 \times \frac{V_{\text {Oxygen }} \times 0.21}{2.0}[34]$ \\
\hline Carbon Dioxide -IRcell - C02 & $\%_{C O_{2}}=0.32 \times V_{C O 2}+0.4[22]$ \\
\hline Combustible 4P-50 & $\%_{L E L}=\frac{0.037}{5.0} V_{\text {Combust }}+1.65[23]$ \\
\hline Temperature - TMP36GRT & $T\left({ }^{\circ} C\right)=100 \times V_{\text {Temperature }}+50[10]$ \\
\hline Humidity - HIH5030 & $\%_{\text {Humidity }}=\frac{0.0062 \times V_{\text {Humidity }}}{3.3}-25.81[35]$ \\
\hline Pressure - NPB-DPNN030PA & $P(k P a)=\frac{0.004 \times V_{\text {Pressure }}}{3.3}+10[36]$ \\
\hline
\end{tabular}

\subsubsection{Sensor Voting}

The pressure sensors in the chassis are critical for maintaining the positive pressure differential needed to meet the A/NZ60079.29 standard [12]. The sensor hardware is triply redundant and has numerous failure indicators. To prevent a sensor failure from compromising the positive pressure explosion protection system, a sensor voting algorithm is needed. The voting algorithm needs to identify faulty/failing sensors and remove them from the signal chain. The voting system also needs to monitor the failure indicators.

To check that the hardware is functioning correctly, an independent reference voltage is passed into the ADC. The ADC reading of the reference pin should fall within pre-determined thresholds. If the reading is outside of these thresholds, this indicates that there is a problem with the sensor hardware.

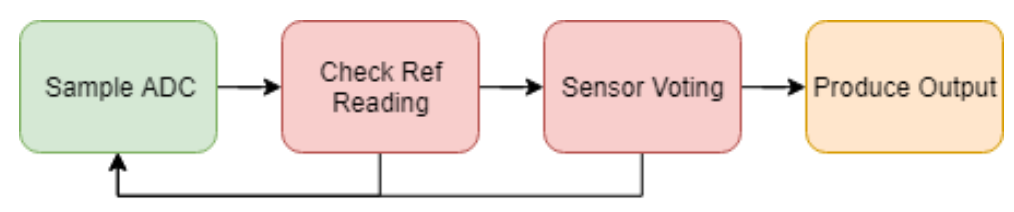

Figure 8.3: The data flow diagram for reading and voting the data from the pressure sensors. 
Figure 8.3 shows the data flow for reading the pressure sensors. The sensor system initially checks the reference voltages. If the reference voltage check is ok, the system then using a sensor voting algorithm to produce an output. If the reference voltage check, or voting algorithm fails, the ADC is re-sampled.

Initially, the voting algorithm takes an average of all the sensor readings. Each sensor reading is then compared to the initial average. The NPB pressure sensors are expected to be accurate within $2 \%$ of one another. If an individual reading is more than $\pm 2 \%$ away from the initial average, the reading is ignored. The average is then re-calculated using only the readings that fall within the $2 \%$ error band. Listing 8.12 shows the function used for completing the sensor voting.

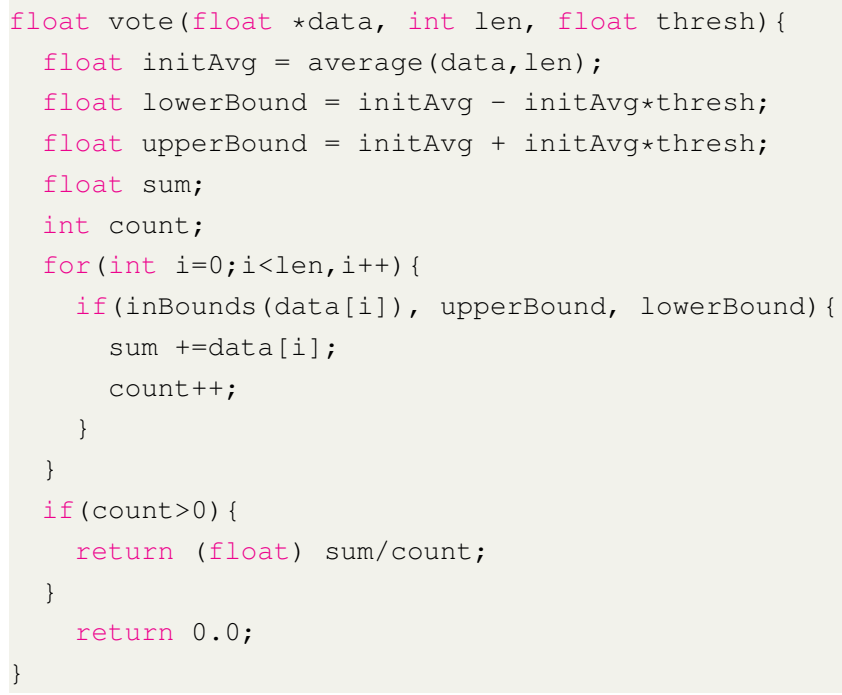

Listing 8.12: The function for voting on the pressure sensor outputs.

The voting algorithm can fail if none of the readings fall within the $2 \%$ error band. In this case the voting method will return 0 and emit a fault code. The software system will then re-sample the ADC and re-complete the sensor voting algorithm with a $4 \%$ error band. 


\subsubsection{Positive Pressure Control System}

The positive pressure explosion prevention system is one of the critical explosion protection methods in HADES. To meet the A/NZ60079.29 standard, the chassis must maintain an inert positive pressure differential of at least $50 \mathrm{~Pa}$. In order to be considered inert, the atmosphere inside the chassis must also contain less than $1 \%$ oxygen.

The chassis is initially filled from an external source of nitrogen. Once in operation, the internal pressure is maintained with an onboard tank. The sensor systems measure the pressure, temperature and oxygen concentration inside and outside the chassis. Three solenoids are employed to control the gas flow, from the internal tank and in/out of the chassis. The implementation of the positive pressure system hardware is covered in Section 3.3 and the sensor systems are covered in Section 5.2.3.2.

The internal and external gas pressure sensors have a $3 \mathrm{~s}$ response time, while the solenoids can respond in $50 \mathrm{~ms}$. As the sensors are slow compared to the actuators, a traditional linear control system will not work. To control the gas pressure in the chassis, a look ahead control system is used. The control system calculates the amount of gas needed to reach the target pressure differential, then activates the purge or tank solenoid to allow the estimated amount of gas to pass. The measured pressure differential $\left(\Delta P_{a}\right)$ is given by,

$$
\Delta P_{a}=P_{c}-P_{e x t},
$$

where $P_{\text {ext }}$ is the external chassis pressure and $P_{c}$ is the internal chassis pressure. To prevent measurement errors and extraneous readings, the pressure measurements pass through a sensor voting and condition system. The error $\left(\Delta P_{E}\right)$ between the actual pressure differential and the target pressure differential $\left(\Delta P_{t}\right)$ is given by,

$$
\Delta P_{E}=\Delta P_{t}-\Delta P_{a}
$$


The ideal gas law (Eq. 8.6) gives the number of the moles of gas to be added (n),

$$
n=\frac{\Delta P_{E} V}{R T},
$$

where, $\mathrm{V}$ is the volume of the chassis, $\mathrm{R}$ is Boltzmann constant $\left(1.380 \times 10^{-23}\right.$ $\mathrm{m}^{2} \mathrm{~kg} \mathrm{~s}^{-2} \mathrm{~K}^{-1}$ ) and $\mathrm{T}$ is the temperature of the chassis. The volume of the chassis is $0.048 \mathrm{~m}^{3}$. The temperature of the chassis is measured using the internal sensor system. The incoming gas will cool the chassis as it expands from the tank. However, this temperature change is ignored as the solenoid is only open for very short amounts of time. Using the tank-chassis pressure differential and the valve flow coefficient $\left(K_{v}\right)$ of the solenoid, the gas flow rate in standard $\mathrm{gs}^{-1}$ can be given by,

$$
M=\frac{5}{18} \cdot \frac{K_{v} \rho_{g}}{0.0019 \sqrt{\rho_{g} T / P_{c}\left(P_{t}-P_{c}\right)}},
$$

where $T$ is the temperature of the chassis and $\rho_{g}$ is the standard density of the gas [46]. The tank and outlet solenoids have a $K_{v}$ of 0.017 and nitrogen gas has a density of $1.2506 \mathrm{~kg} / \mathrm{m}^{3}$. The flow rate and amount of gas needed can be used to calculate the activation period of the solenoid $(t)$ with Eq. 8.8.

$$
t=\frac{M \cdot n}{m_{n}}
$$

where $m_{n}$ is the molar mass of nitrogen gas $(28 \mathrm{u})$. If the chassis is overpressure, the purge solenoid is opened and if the chassis is under pressure, the tank solenoid is opened. The Zilch thread for controlling the positive pressure system is shown in Listing 8.13.

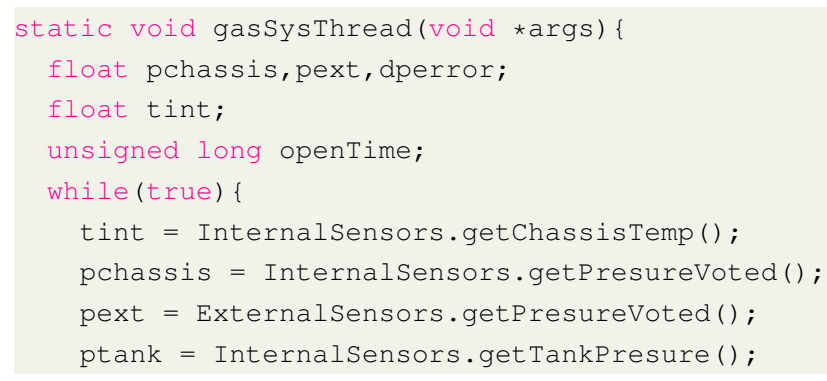




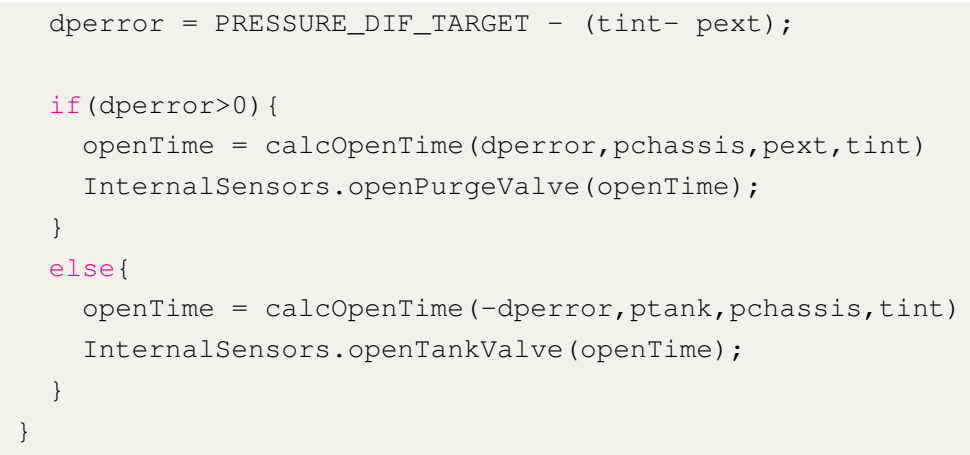

Listing 8.13: The Zilch thread for controlling the positive pressure regulation system.

The pressure system thread reads the system pressures and temperatures from the two sensor system classes. The pressure differential error (dperror) is then calculated using Eq. 8.4 and Eq. 8.5. If the system is over pressure, the valve open time is calculated using the chassis pressure and the external pressure. If the system is under pressure, the valve open time is calculated using the tank pressure and the chassis pressure.

As Zilch can context switch during delays, some delay times can be inaccurate. Too accurately time the solenoid open time, a single shot 16-bit timer is used. To implement the timer the, IntervalTimer Arduino Library is used. Listing 8.14 shows the code for opening the solenoid and starting the timer. When the timer finishes, an interrupt is triggered, calling an ISR that closes the solenoid. To prevent over pressurisation, the timer period is bounded to a maximum of $2 \mathrm{~s}$. To prevent the control system from measuring the gas pressure while the values are open, the solenoid activation methods are blocking.

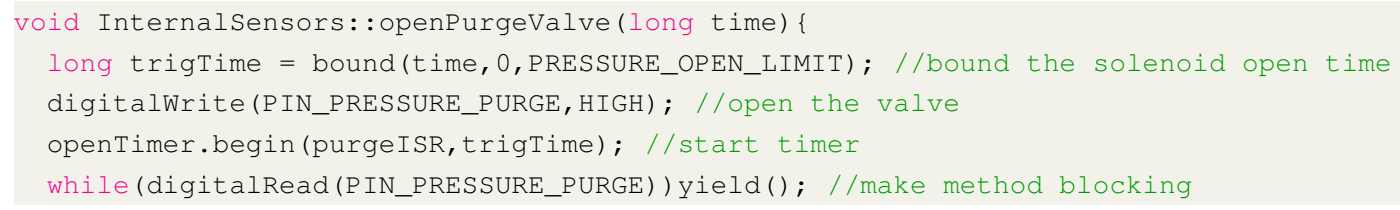


opentimer.end ();

$7\}$

Listing 8.14: The method used for controlling the activation time of purge solenoid.

\subsubsection{Watchdog Microcontroller}

The watchdog microcontroller monitors the condition of the main microcontroller and can intervene if it detects a fault. The watchdog microcontroller is connected to the main microcontroller with three digital IO lines and the I2C bus. The software watchdog on the main microcontroller is reset every $500 \mathrm{~ms}$ in normal operation. If the watchdog is not reset for 2 seconds it will timeout; when this timeout occurs, the WD pin is pulled low. The watchdog controller monitors the WD line and assumes control of the system on a negative edge.

When a fault occurs, the watchdog controller pulls the E-STOP line low to put the robot into a safe state, it then attempts to hardware reset the main microcontroller using the $\overline{R S T}$ line. While the main microcontroller is rebooting, the watchdog controller assumes the role of I2C master. This allows the watchdog controller to maintain system control. As the watchdog microcontroller only has $4 \mathrm{kB}$ of memory, the $\mathrm{I} 2 \mathrm{C}$ command set is minimised to critical safety features [28]. The reduced instruction set allows the watchdog controller to control system components, but does not have the instructions for collecting and managing data. After the main microcontroller has reset, the rel line is asserted to release control from the watchdog controller. 


\subsubsection{Summary}

The embedded code on the main microcontroller interfaces with the sensor systems and drive motors with five different Arduino classes. The Arduino classes keep the code modular and prevents a fault from cascading throughout the complete code base. Each of the key code functions are run with a Zilch thread which allows these functions to be paused and resumed throughout program execution. Running each function in a separate thread also keeps the functions independent and prevents a fault in one functional block from affecting the complete system.

\subsection{Power Controller}

The power controller monitors and controls the power supply, regulation and distribution inside HADES. Accurate monitoring and control is necessary as bad power levels can damage system components and lead to system-wide failures.

The power controller is the first of the controllers to receive power, thus it governs the start-up process for the remainder of the system components. A controlled start-up is necessary as it allows each of the system components to be checked before the start of subsequent components. This prevents a fault from cascading through the system, damaging multiple components. Figure 8.4 shows the decision tree for the start-up of the power controller.

Upon power-up of the power controller, initial validation of the control system batteries is completed. If the control batteries fall within safe operating parameters, the main microcontroller and auxiliary microcontroller will be notified via an interrupt on the fault line. If the batteries do not pass their validation tests, the power controller will enter a sleep mode, awaiting user interaction. 


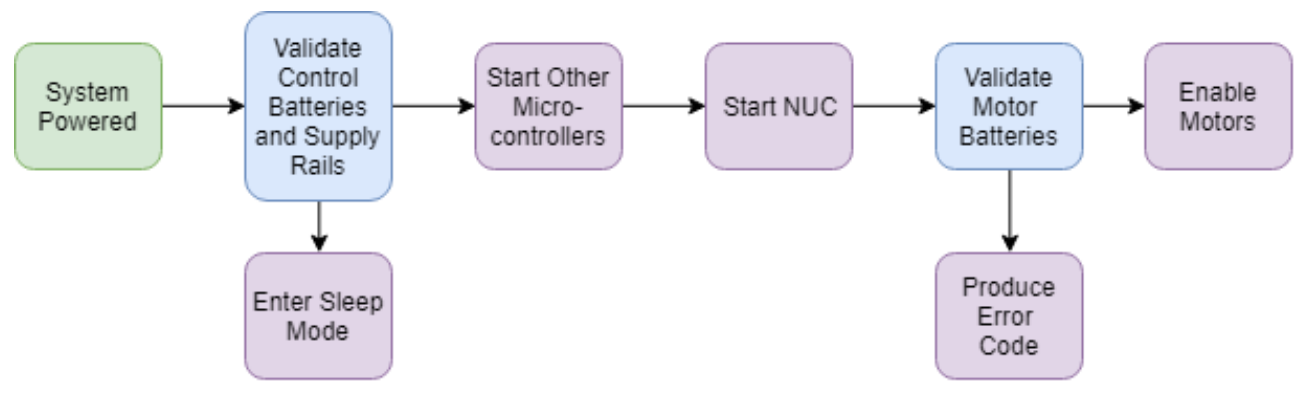

Figure 8.4: The start-up sequence for the power controller.

Once the main microcontroller has successfully booted, the power controller will supply power to the NUC, allowing it to start. Finally, in the start-up sequence, the controller will check the status of the motor batteries and determine if they are within safe operating parameters and if so the ECSs and motors are enabled. Following the enabling of the motor batteries, the controller will enter normal operation mode.

During normal operation, the power controller monitors the condition of the batteries and supply rails. If there is a fault, the power controller will take action to safely isolate the fault and inform the operator by pulling the fault line low for the main microcontroller to service. Two Arduino classes are used in the power controller to simplify the code base, one for the battery management system and one for the supply rails.

\subsubsection{Battery Management System}

The lithium polymer batteries used in HADES can become critically damaged, or even explode if unsafe operating conditions are reached (Section 6.2.2). To prevent this from occurring, a battery management system is implemented. The battery management needs to monitor the cell voltages, battery current and temperature for unsafe conditions. If unsafe conditions are reached, the BMS system should isolate the battery from its load. 
The BMS software is implemented as an Arduino class. Six BMS objects are used, one for each of the batteries. The BMS object holds all the data for its assigned battery as well as the functions for updating the data. Listing 8.15 shows the $\mathrm{C}++$ header file for the public functions in the BMS class.

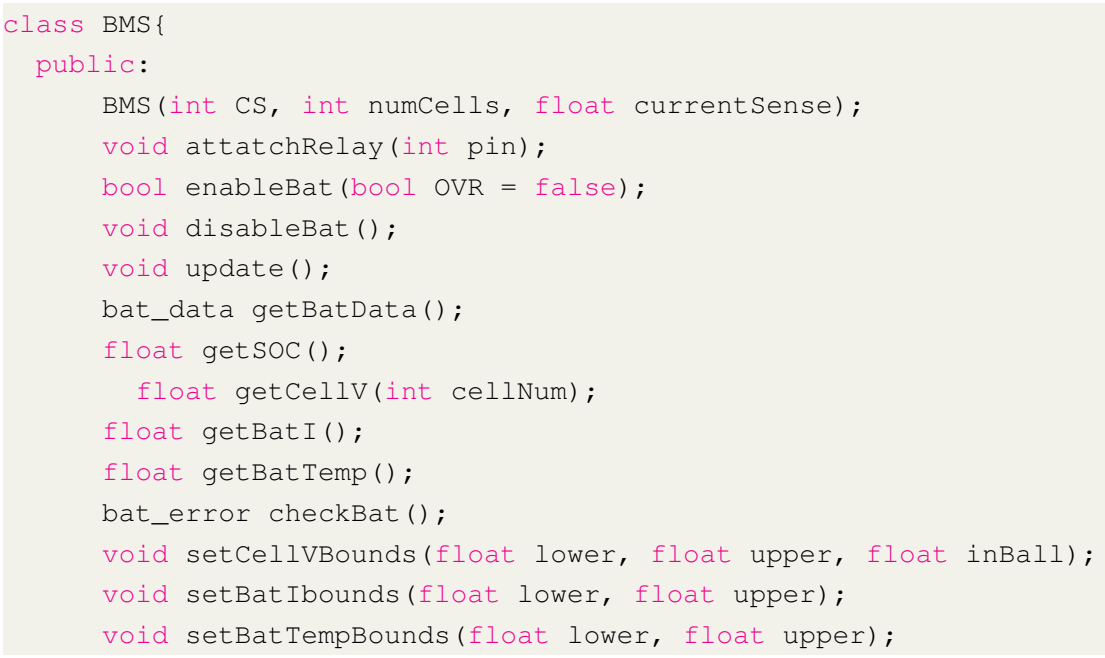

Listing 8.15: The public methods of the BMS class used to monitor the batteries in HADES.

During safe operation, the cell voltages in a battery should all be balanced, however, as the battery becomes unhealthy, the cell voltages will begin to deviate. Monitoring the deviation of the cell voltages and ensuring they remain within safe working conditions will increase the battery life. During safe working conditions, the cells in a Li-Po battery should not deviate by more than $0.15 \mathrm{~V}$. To check the deviation of each cell, the minimum and maximum values are found, then the difference is taken.

Monitoring the State of Charge (SOC) of the batteries allows the operator to gain an understanding of the remaining battery life and therefore remaining operation time. There are many methods for monitoring SOC, the most common and simplest is Coulomb counting [19]. The SOC equation for Coulomb 
counting is given as,

$$
S O C(t)=S O C(t-1)+\frac{I(t)}{Q_{n}} \Delta t
$$

where $S O C$ is the state of charge, $I$ is the current draw from the battery, $t$ is the time and $Q_{n}$ is the charge of a Coulomb. To accurately track the SOC, the Coulomb counting calculation needs to be run as fast as possible, when running on the SAMD21G18 microcontroller a sample rate of $854 \mathrm{~Hz}$ is achieved.

If the battery does become unsafe, a fault code is generated which is then reported to the main microcontroller with the I2C interface. If the battery has a shut-off relay, the relay will be disconnected. If there is no shut off functionality, the power controller will attempt to reduce the load by shutting down system components. If the operator deems it safe to continue operating, the battery isolation can be overridden.

\subsubsection{Voltage Rail Monitoring}

HADES has universal 12 and $5 \mathrm{~V}$ rails that supply the majority of the system components (Section 6.2.3). Each of the rails has hardware for monitoring the current and voltage of the regulator outputs. The supply rail is powered by dual redundant regulators, which can be selected with a power management switch. A software system is needed to monitor the voltage rails and switch between regulators if fault conditions are reached.

The supply rail software is implemented with an Arduino class. Two classes are implemented, one for each of the global supply rails $(12 \mathrm{~V}$ and $5 \mathrm{~V})$. The HADES_RAIL class holds all the functions and data needed to monitor and control a supply rail in HADES. Listing 8.16 shows the declaration of the public functions for the HADES RAIL class. 


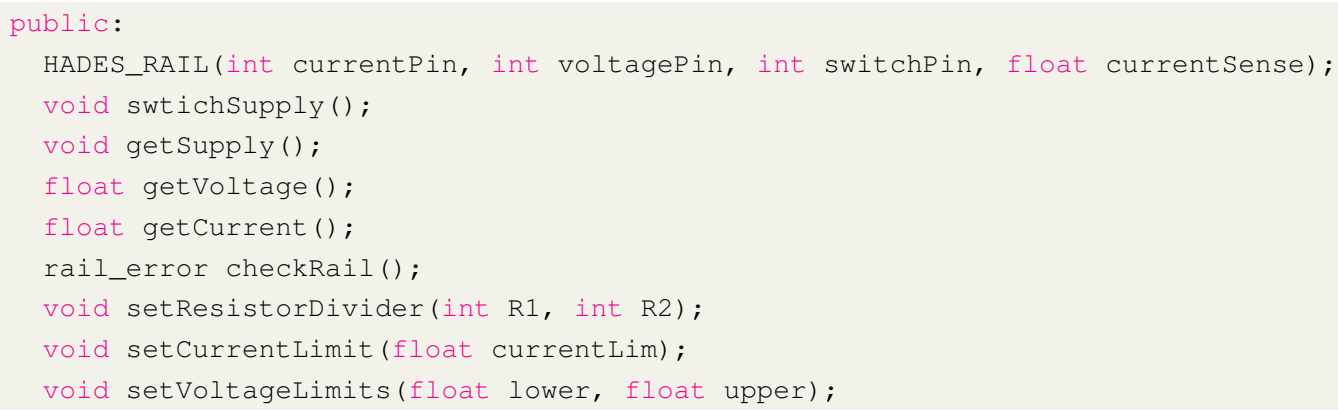

Listing 8.16: The header of the $\mathrm{C}++$ class for monitoring the supply rails.

\subsubsection{Summary}

The code on the power controller monitors the condition of the batteries used in HADES. Each battery management system has a dedicated software class to store and monitor the data from the battery's sensors. If the battery breaches safe operating conditions, the BMS software will automatically act to reduce the load on the battery. The supply rails are also monitored by embedded code on the power controller. The power controller will automatically switch between the supply rails sources if the rail becomes unstable. The power controller can also issue instructions to other sub-systems to reduce their load if the regulator is overloaded. 


\section{Chapter 9}

\section{Evaluation and Discussion}

Access to a working underground mine will not be granted until HADES has passed the compliance testing for the safety standards identified in Section 2.3. Therefore, to evaluate HADES, a series of laboratory and field tests were undertaken; the results of these tests are presented in this chapter.

\subsection{Locomotion}

The locomotion system needs to be able to move HADES though the terrain inside a post-disaster underground mine. Section 2.1 identified that the expected terrain could be categorised into five broad categories; compact dirt/gravel, loose gravel/dirt, train tracks, small boulders (50-200 mm diameter) and large boulders (>200 mm diameter). Four of the five terrain categories were tested on flat ground and whilst ascending and descending various inclines. Train tracks could not be tested as a suitable test location could not be found.

To succeed in locomotion tests, HADES should still be able to navigate as the operator intends, even if one or more of the locomotion systems are operating 
at reduced power or have failed. Additionally, the operator needs to be able to control HADES in the confines of an underground mine. The locomotion test results of the four terrain categories tested are detailed below.

\subsubsection{Compact Dirt/Gravel and Small Boulders}

The terrain in the mine entry shaft is likely to consist of gravel and small boulders (Section 2.1). HADES must therefore be able to navigate this terrain with relative ease at the expected cruising speed. To test this, HADES was tested on a surface with compact gravel and small boulders. The trails were conducted on flat ground and on a $20^{\circ}$ incline. A test was considered a success if HADES was able to travel at $1.4 \mathrm{~ms}^{-1}$ (cruising speed) over the terrain.

Table 9.1: Compact gravel and small boulder locomotion success rates.

\begin{tabular}{|l|l|l|}
\hline & Compact Gravel & Small Boulders \\
\hline Flat ground & $100 \%$ & $100 \%$ \\
\hline Descending & $100 \%$ & $100 \%$ \\
\hline Ascending & $100 \%$ & $100 \%$ \\
\hline
\end{tabular}

Table 9.1 shows the results from the gravel and small boulder trails. HADES was able to navigate both terrain types, for all three topographies successfully. During the small boulder tests, boulders would occasionally scrape against the bottom of the chassis. However, the locomotion systems had enough power to pull the chassis over these.

The current supplied to the ESCs and the motor currents are both recorded. Figure 9.1 shows the motor and battery currents plotted together for flat ground travel. This figure highlights the buck/boost feature of the ESC (Section 6.1) as the battery currents are significantly lower than the motor currents. 


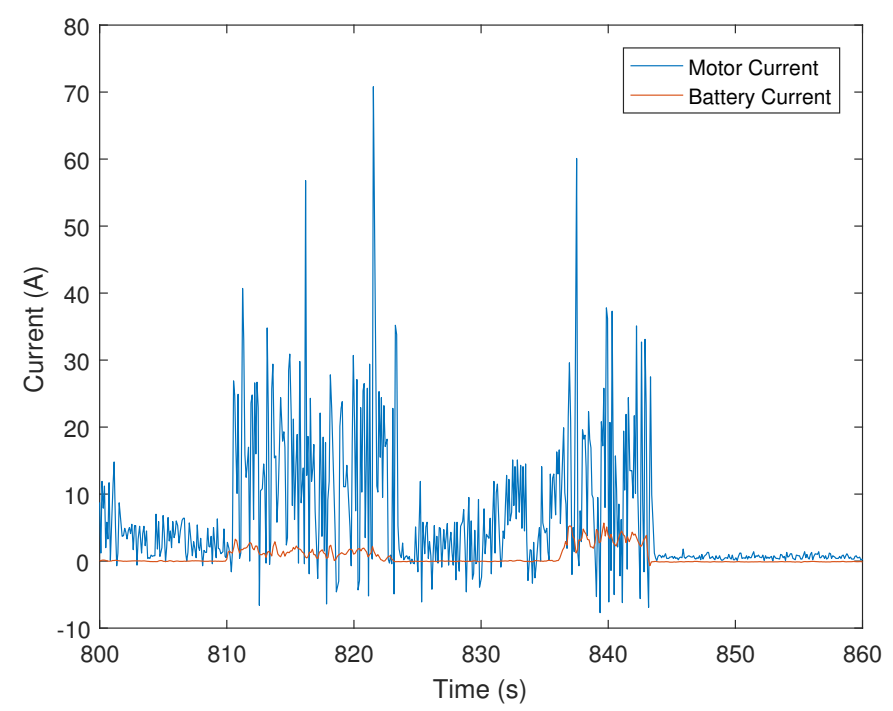

Figure 9.1: A plot of motor current vs. battery current for flat ground locomotion.

\subsubsection{Loose Gravel}

The loose gravel tests were completed on pieces of $15 \mathrm{~mm}$ diameter gravel, forming a gravel bank which varied from between $0.2 \mathrm{~m}$ and $3 \mathrm{~m}$ deep. HADES was tested travelling along flat ground and while ascending and descending a $15^{\circ}$ incline. If HADES was able to travel at $0.7 \mathrm{~ms}^{-1}$ (half cruising speed) over the terrain, the test was considered a success; each terrain was trailed ten times. Table 9.2 shows a summary of the results from each trail.

Table 9.2: The success rates of the loose gravel locomotion tests.

\begin{tabular}{|l|l|}
\hline Terrain & Success Rate \\
\hline Flat Ground & $100 \%$ \\
\hline Ascending & $0 \%$ \\
\hline Descending & $100 \%$ \\
\hline
\end{tabular}

HADES was able to traverse over loose gravel when the terrain was flat. 
During these trails, the Whegs would sink into the gravel about $50 \mathrm{~mm}$. However, the drive systems had enough power the pull Whegs out of the gravel and continue moving.

When ascending a gravel incline, the Whegs would also sink into the gravel. When travelling up a slope, this caused the front of the chassis to bottom out. When the front of the chassis bottomed out, the Whegs would lose traction and dig further into the gravel, causing the remainder of the chassis to bottom out. Once HADES bottomed out, the Whegs would spin freely and not gain any traction, preventing movement. Figure 9.2 shows HADES bottomed out when climbing a loose gravel surface.

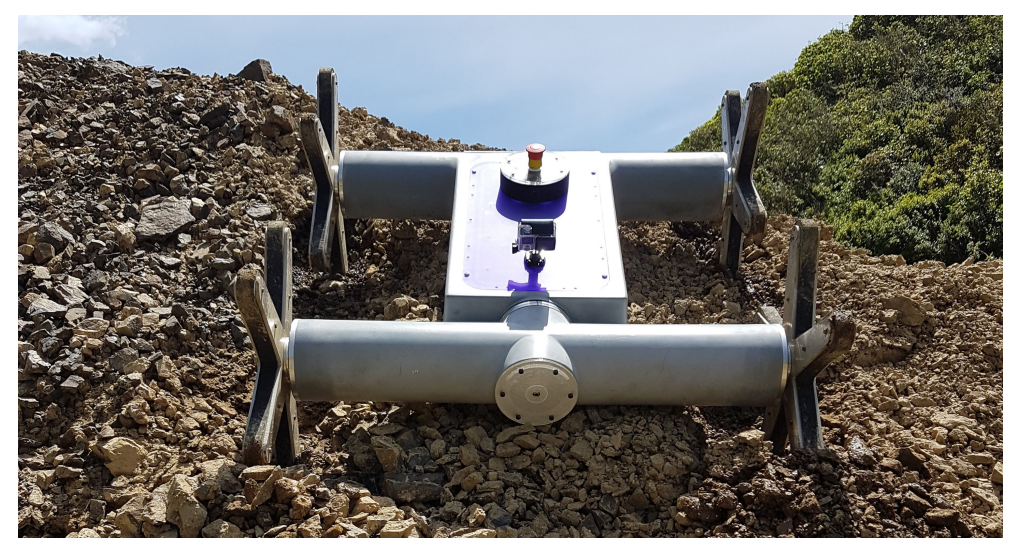

Figure 9.2: HADES bottomed out when travelling up a loose gravel slope due to the Whegs digging into the gravel.

Although HADES could not ascend the inclines, the drive systems always had enough power to reverse out of the hole caused by the spinning Whegs. HADES was able to descend all the loose gravel inclines during testing. During $40 \%$ of the descent trails, the Whegs still sunk into the gravel causing the chassis to bottom out. However, in such instances, the drive systems were able to pull HADES along. 


\subsubsection{Large Boulders}

The large boulder test was completed on rocks between $150 \mathrm{~mm}$ and $500 \mathrm{~mm}$ in diameter, some of these rocks could be easily moved. The tests involved traversing HADES though this terrain ten times; a success was if HADES could make it over the terrain without physical intervention. Different paths were taken each time to ensure that differing terrain was covered.

All but two of the tests were successful, where HADES managed to navigate through the boulders. One of the tests failed when HADES encountered a boulder that was approximately $400 \mathrm{~mm}$ in diameter and could not get a purchase to climb. While an alternative route was found around the large boulder, if the terrain largely comprised of boulders greater than $400 \mathrm{~mm}$ HADES would have extreme difficulty navigating.

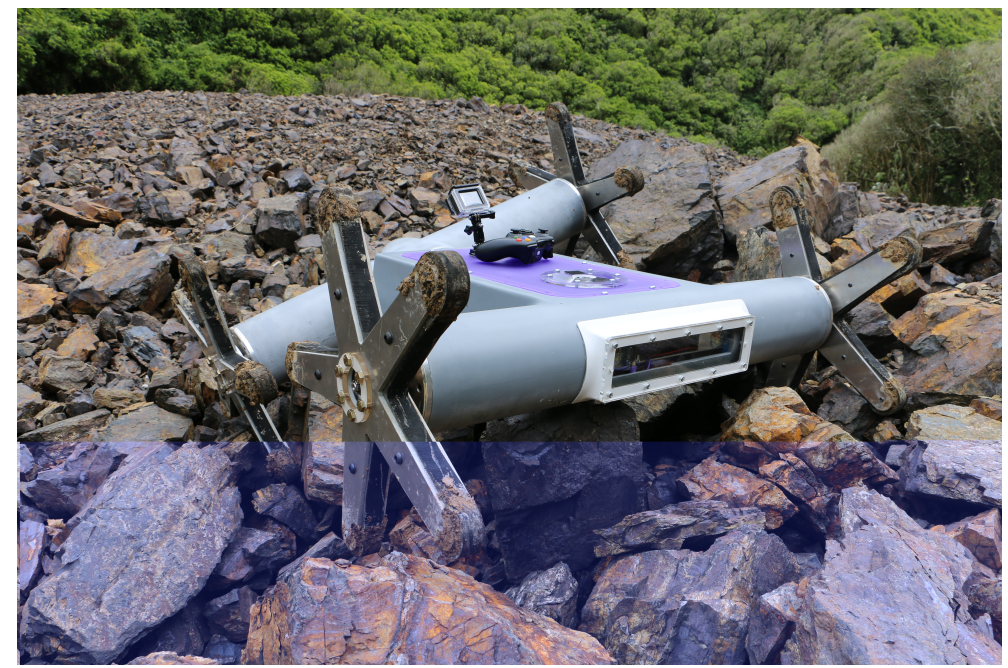

Figure 9.3: HADES travelling over a large bolder field with the two front Whegs not in contact with the ground.

The second test failed when HADES was cresting an obstacle and bottomed out. With the chassis held off the ground, the Whegs did not have sufficient 
traction to move the chassis. During all the tests HADES bottomed out frequently, this was not an issue as the rear arm pivot allowed at least one Wheg to be in contact with the ground, and HADES was still able to move over the terrain with that one Wheg, until another Wheg could contact the ground. Figure 9.3 shows HADES bottoming out when travelling over large boulders. Figure 9.3 also highlights the rear arm pivoting to allow the rear-right Wheg to contact the ground when the front Whegs cannot.

\subsubsection{Reduced Locomotion Power}

HADES should still be able to navigate with failed/damaged locomotion components. The locomotion systems have two major failure modes, loss of power causing free-run and a mechanical fault causing seizure of the output shaft. These two failure modes are tested by either disconnecting the ESC from power using the battery management system (Section 6.2.2) or braking the motor using the ESC.

To test the reduced locomotion power performance, the rear locomotion systems were either disconnected or locked. The robot was then driven in both directions to simulate a fault in the front locomotion system. During these tests, the rear arm pivot was locked. Twelve tests were carried out in each direction; with one or two locomotion systems compromised, the wheels unpowered or locked, and either ascending or descending sloped ground or along flat ground. All the reduced locomotion system tests were performed on a compacted gravel surface.

The purpose of these tests was to ascertain if the compromised locomotion systems could still move HADES. A test was considered a success if HADES was able to travel along the surface at $0.35 \mathrm{~ms}^{-1}$ (a quarter of the expected cruising speed). Tables 9.3 and 9.4 show the test results, for the flat ground and incline reduced locomotion trails, respectively. 
Table 9.3: The success/ failure table for different scenarios of reduced locomotion testing on flat ground.

\begin{tabular}{|l|l|l|}
\hline Test Type & Forward Travel & Reverse Travel \\
\hline 1 wheel locked & Success & Success \\
\hline 2 wheels locked & Failure & Success \\
\hline 1 wheel unpowered & Success & Success \\
\hline 2 wheels unpowered & Success & Success \\
\hline
\end{tabular}

HADES was able to travel over flat ground for seven out of the eight reduced locomotion tests and was successful for ten out of the sixteen incline tests. The primary failure mode of the locomotion tests was the lack of traction for the drive wheels. When there was sufficient load from the disabled locomotion systems, the driven Whegs would lose traction.

Table 9.4: The success / failure table for different scenarios of reduced locomotion testing on sloped ground.

\begin{tabular}{|l|l|l|l|l|}
\hline Test Type & \multicolumn{2}{|l|}{ Forward Travel } & Reverse Travel \\
\hline & Ascending & Descending & Ascending & Descending \\
\hline 1 wheel locked & Success & Success & Success & Success \\
\hline 2 wheels locked & Failure & Failure & Failure & Success \\
\hline 1 wheel unpowered & Success & Success & Failure & Success \\
\hline 2 wheels unpowered & Failure & Failure & Success & Success \\
\hline
\end{tabular}

During the tests where one of the drives systems was disconnected or locked, HADES would turn in an arc towards the side of the faulty system due to the lack of drive power on one side. The operator was able to compensate for this drift with the $\mathrm{Xbox}{ }^{\mathrm{TM}}$ controller (Section 7.1.3.1).

When two of the wheels were locked or disconnected, HADES could travel backwards, but not forwards. This was because when HADES was travelling backwards, the drive wheels are pushing the disabled wheels off the ground 
and not dragging them into the ground.

\subsubsection{Manoeuvrability}

HADES needs to be operated remotely in the confines of an underground mine. To simulate this, a series of tests were undertaken to determine the manoeuvrability of HADES in a confined space. The tests were to ascertain the turning circle of HADES, as well as the controllability of HADES at different speeds.

To test the turning circle, HADES was made to perform a full skid turn on different surfaces. A full skid turn is when the motors on each side of the robot are rotated in opposite directions. HADES was made to complete five full revolutions on each surface, then the diameter of the space needed as well as any lateral displacement was measured.

Table 9.5: The turning circle test results for HADES on different terrains.

\begin{tabular}{|l|l|l|}
\hline Terrain Type & Turing Circle & Lateral Displacement \\
\hline Compact Dirt & $850 \mathrm{~mm}$ & $<100 \mathrm{~mm}$ \\
\hline Loose Gravel & $900 \mathrm{~mm}$ & $<100 \mathrm{~mm}$ \\
\hline Small Boulders & $970 \mathrm{~mm}$ & $150 \mathrm{~mm}$ \\
\hline Large Boulders & $1500 \mathrm{~mm}$ & $400 \mathrm{~mm}$ \\
\hline
\end{tabular}

Table 9.5 shows the results for the turning circle test. HADES was able to complete $3 / 4$ of the turning tests within a $1000 \mathrm{~mm}$ diameter circle with minimal lateral displacement. To complete a turn on the large boulders, HADES required a much larger area. This was due to the Whegs catching on the boulders when turning and forcing HADES away from the centre of rotation. Instead of skid turning on large boulders, the operator should perform multi-point turns as these do not cause unwanted lateral displacement.

To meet the overarching requirements (Section 2.4), HADES needs to be able 
to travel at $1.4 \mathrm{~ms}^{-1}$ and be controllable. To test this, HADES was run in a straight line at different speeds until HADES reached $1.4 \mathrm{~ms}^{-1}$ and the smoothness of running and controllability were recorded.

To run HADES in a straight line, all four motors were set to run at equal speed with closed loop control mode (Section 6.1). The amplitude of the vertical oscillations of the main body and the deviation from straight line travel with no operator were recorded, these can be seen in Table 9.6.

Table 9.6: The amplitude of the vertical oscillations and the displacement from straight line travel for different travel speeds.

\begin{tabular}{|l|l|l|}
\hline $\begin{array}{c}\text { Speed } \\
\mathbf{( m s}^{-1} \mathbf{)}\end{array}$ & $\begin{array}{c}|c| \\
\text { Amplitude of } \\
\text { Vertical Oscillations } \\
(\mathbf{m m})\end{array}$ & $\begin{array}{c}\text { Displacement from } \\
\text { straight line travel } \\
\mathbf{( m )}\end{array}$ \\
\hline 0.25 & 50 & 0 \\
\hline 0.7 & 80 & 0.2 \\
\hline 1.0 & 120 & 0.6 \\
\hline 1.4 & 150 & 1.5 \\
\hline
\end{tabular}

The straight line tests were completed at four different speeds: $0.25,0.7,1.0$ and $1.4 \mathrm{~ms}^{-1}$. The amplitude of the oscillations increased as the travel speed of HADES increased. This was due to the Whegs hitting the ground faster and more frequently, causing bigger and more frequent changes in vertical acceleration. A speeds above $1 \mathrm{~ms}^{-1}$ the vertical oscillations where large enough to vibrate HADES off-course, this is most prevalent at $1.4 \mathrm{~ms}^{-1}$ where HADES travelled $1.5 \mathrm{~m}$ off-course over a $5 \mathrm{~m}$ travel distance. However, when completing the majority of the locomotion trails the operator could compensate for this sideways drift. 


\subsubsection{Locomotion - Discussion and Summary}

In all of the tests performed, the drive system was able to supply enough torque to drive the Whegs the systems were also easily capable of reaching the required speeds. The Whegs were able to complete $67 \%$ of the locomotion trails successfully, however, some design changes could easily improve this.

The majority of the failed tests occurred due to lack of traction. Two main factors caused the loss of traction were not enough grip on the Wheg and the chassis bottoming out. During the controllability trials, the Whegs caused large vertical oscillations at higher speeds.

To improve the ground clearance, the diameter of the Wheg should be increased, however, this will also increase the amplitude of the vertical oscillations. To mitigate this trade-off, the six-spoke rear Whegs should be implemented (Section 4.1). The six-spoke Whegs would allow a larger diameter Wheg to be used without increasing the unwanted vibration.

Increasing the duty cycle of the Whegs would also minimise the unwanted motion, a duty cycle increase would also reduce the probability of the Whegs sinking into soft terrain, as observed in the loose gravel test. However, this would decrease the Wheg's ability to climb over large obstacles. Instead of increasing the duty cycle, the width of the Whegs could be increased. This would increase the contact area with the ground, increasing traction and decreasing the likelihood of a Wheg sinking into soft terrain. Additionally, to improve the grip, a textured tread could be cut into the tip of each spoke, allowing the tip to grip larger obstacles. 


\subsection{Power Consumption}

To meet the run-time requirement, HADES needs to be able to operate for 4 hours on a single battery charge. To test this, the current from the control system and motor batteries was measured with the onboard Hall-effect sensors. Figure 9.4 shows the current consumption from the motor batteries when travelling at $1.4 \mathrm{~ms}^{-1}$.

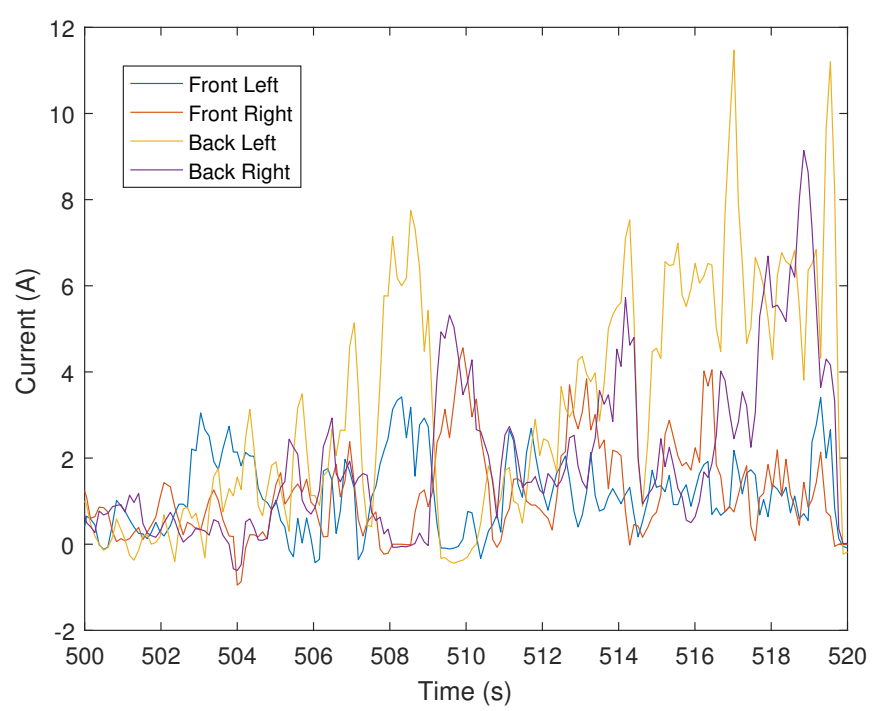

Figure 9.4: The battery current supplied to each driven system when travelling at $1.4 \mathrm{~ms}^{-1}$

The average current drawn from the motor batteries is $1.9 \mathrm{~A}$. At this draw the 12 Ah batteries will be able to supply HADES for 6-hours of operation, exceeding the specification by $50 \%$. The control system battery supplies an almost constant 0.7 A to the control electronics. The largest current variation is due to the switching of the illumination LEDs and BMS relays. Enabling a relay increases the battery draw by $60 \mathrm{~mA}$, and the LED draw can vary between 0 $500 \mathrm{~mA}$ depending on the brightness and system selected. To ascertain battery life, the worst case scenario is used, in which a constant $1 \mathrm{~A}$ is being drawn from 
the control system battery. When drawing this current from a 12 Ah battery, it is expected that the control system will be able to run for 12 hours.

Although the estimated run-time exceeds the specification, there are additional loads that need to be considered. The motor current graph, in Fig. 9.4 above, is for when HADES was traversing flat ground. The current needed for climbing rough terrain will be considerably higher. However, it is likely that when climbing rough terrain HADES will not be in continuous operation, as rough terrain needs a more considered approach. The currents were measured when the batteries were near maximum charge. As the batteries discharge their voltage will decrease, increasing current draw for the same power output.

However, during the locomotion testing, HADES was operated intermittently (75\% duty) cycle for 3 hours. Additionally, during this time the control system was left running. By measuring the cell voltages, an approximation of battery use can be made. After three hours of run-time, the voltage measurements indicated an approximate remaining capacity of $65 \%$ for the motor batteries and $70 \%$ for the control battery. This means that for this use case, the motor batteries could last for up to 9 hours of operation.

\subsection{Chassis Evaluation}

Significant stress was placed upon the chassis when traversing the loose gravel and large boulder fields. However, the chassis withstood all of the locomotion trials. During these trials, the chassis bottomed out numerous times causing it to scrape along the ground. These impacts damaged the bottom of the chassis, as shown in Figure 9.5. However, the damage on the bottom of the chassis is purely cosmetic, no identifiable damage extended past the gel-coat layer of the fibreglass. The motor and rear arm pivots did not distort or break during testing, and inspection after the tests indicated no damage had occurred. The 


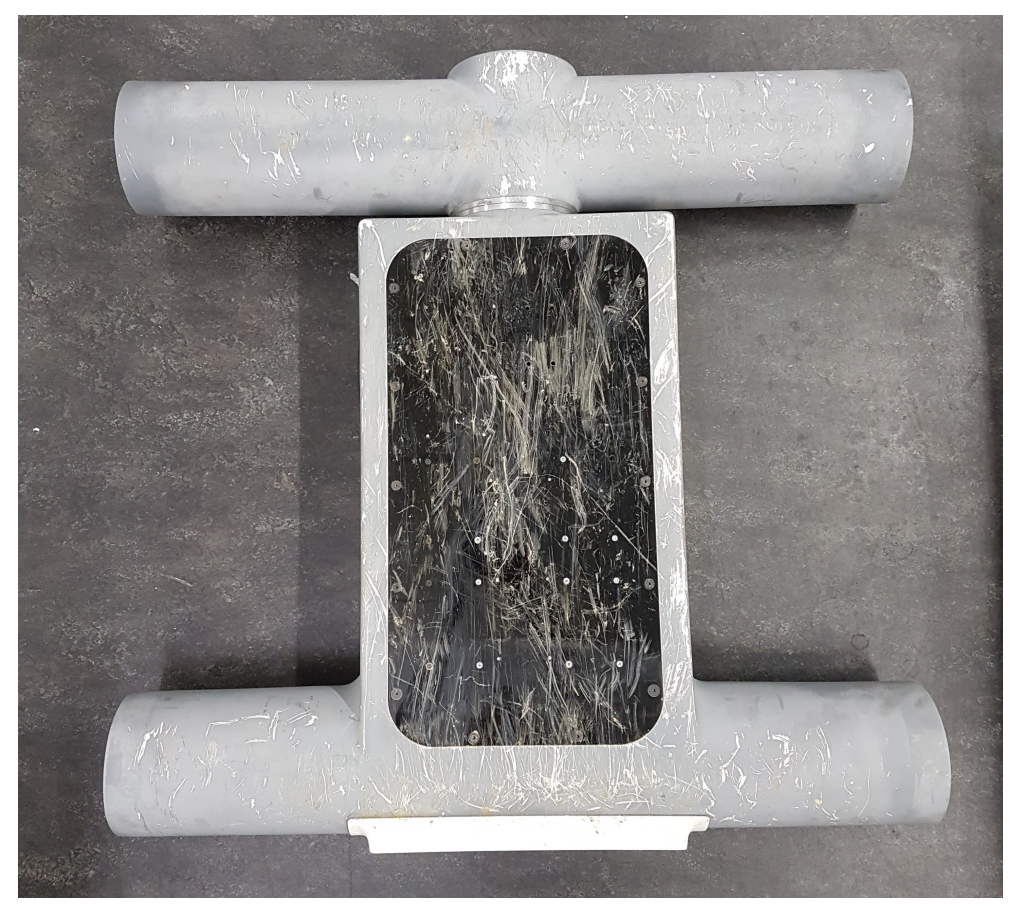

Figure 9.5: The scrapes on the bottom of the HADES chassis from the locomotion tests.

surface damage that occurred to the chassis during testing was mostly from large rocks.

HADES is expected to be able to climb over steel train tracks. After a disaster, these train tracks could potentially be broken and have sharp protruding edges. As steel is much harder than the rocks used for the locomotion testing, the chassis could potentially be damaged if HADES is required to traverse the broken steel. To increase the surface hardness of HADES, a tougher gel-cost could be used. Automotive paint could also be applied to provide additional scratch resistance. These two features would toughen the outer skin of HADES with minimal extra weight, but would however, significantly increase the cost. 


\subsubsection{Rear Arm Pivot}

The arm pivot proved useful when navigating the terrain tested in the locomotion tests (Section 9.1). It allowed the rear Whegs to gain traction when they would otherwise be off the ground. After the trials, the rear arm pivot was disassembled to check for galling and wear (Section 3.2).

Significant galling was found on the plates of the rear arm (Figure 9.6). This is caused by the radial loads of the rear arm causing the faces to be nonparallel. When the faces are nonparallel, the load is placed on a much smaller area around the outside of the face. The smaller load area means that the surface pressure is much higher than expected, causing galling. One of the bolts holding the pivot to the chassis also worked loose, this caused further damage to the pivot plates.

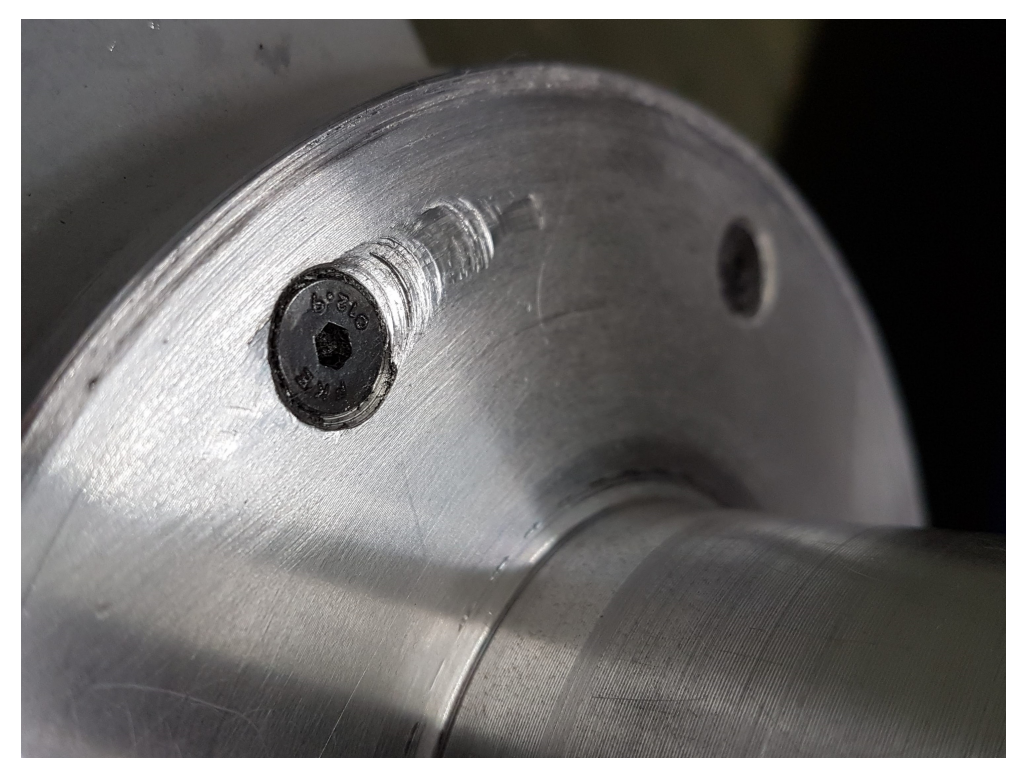

Figure 9.6: The damage to the running faces of the rear arm pivot assembly.

The barrel of the pivot assembly was not damaged during the testing. However there was significant wear around the steel belt pulley flange. The aluminium face that was in contact with the pulley flange had worn away by 
$0.26 \mathrm{~mm}$. This caused the pivot to gain axial play, further exacerbating the galling on the faces.

The rate of wear and galling was significantly higher than predicted for the aluminium pivot assembly. For future revisions, a bronze and steel pivot is recommended (Section 9.3.1.1), as it mitigates the problems found in the aluminium design. Locktite ${ }^{\mathrm{TM}}$ should be added to all the mounting bolts to prevent them from working loose.

\subsubsection{Brass and Steel Rear Arm Pivot}

Steel and Bronze are harder and have a much lower coefficient of friction when compared to aluminium and so are much less likely to gall under high loads [70]. Bronze can be impregnated with oil at manufacture to form Oilite and so can form self lubricating bushings that require very little maintenance [26]. By redesigning the pivot assembly from steel and bronze, it will have a much longer operational life and will require considerably less maintenance.

The main dimensions of the steel and brass pivot assembly would be kept the same as the aluminium design. The main body of the two steel pivot parts could be made from $3 \mathrm{~mm}$ AISI 1018 steel, a thinner wall thickness than the aluminium pivot as steel 1018 steel is approximately 2-3 times stronger than 2014 grade aluminium [70]. Reducing the wall thickness of the pivot assembly also keeps the weight down, as steel is typically 2.5 times denser than aluminium.

The running surfaces of the pivot could all rotate on Oilite bushings. Three bushings would be used, two to support the rotational pivot and one to separate the rear and forward flanges. The forward Oilite bushing would have a flange that rides against the steel flange on the belt pulley, spreading the pressure from the axial load and preventing a steel on steel running surface. All three of the bushings and the steel parts can be seen a cross section view of the bronze and steel pivot assembly, shown in Figure 9.7. To seal the assembly, the 


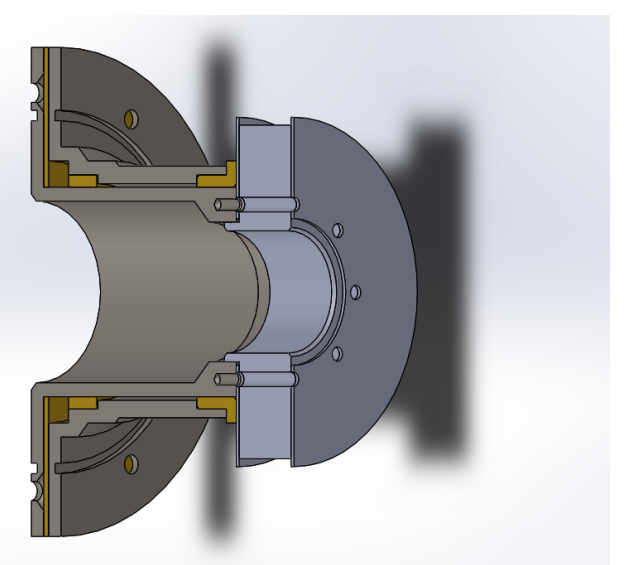

Figure 9.7: A cross section of the steel and bronze rear arm pivot.

same O-rings and low pressure lip seal would used as in the aluminium design. The steel and bronze assembly would increase the wight of HADES by $300 \mathrm{~g}$, but would considerably extend the operating life and reliability of the pivot assembly.

\subsubsection{Ingress Protection}

HADES needs to meet the IP68 standard to protect the internal components from water and dust ingress. HADES must also be buoyant to allow it to navigate a flooded mine. Upon taking delivery of the chassis from the manufacturer, several manufacturing faults were identified that could cause a leak in the chassis.

The motor mounting points had several large crease marks in the sealing surface from the moulding process. The creases would allow water to wick behind the O-ring seal and into the chassis. Secondarily, some of the mounting bolt holes for the motor and rear arm pivots had not been fibreglassed over. This would also water to flow through the thread of the bolt and into the chassis. One of the irregular sealing surfaces can be seen in Figure 9.8. 


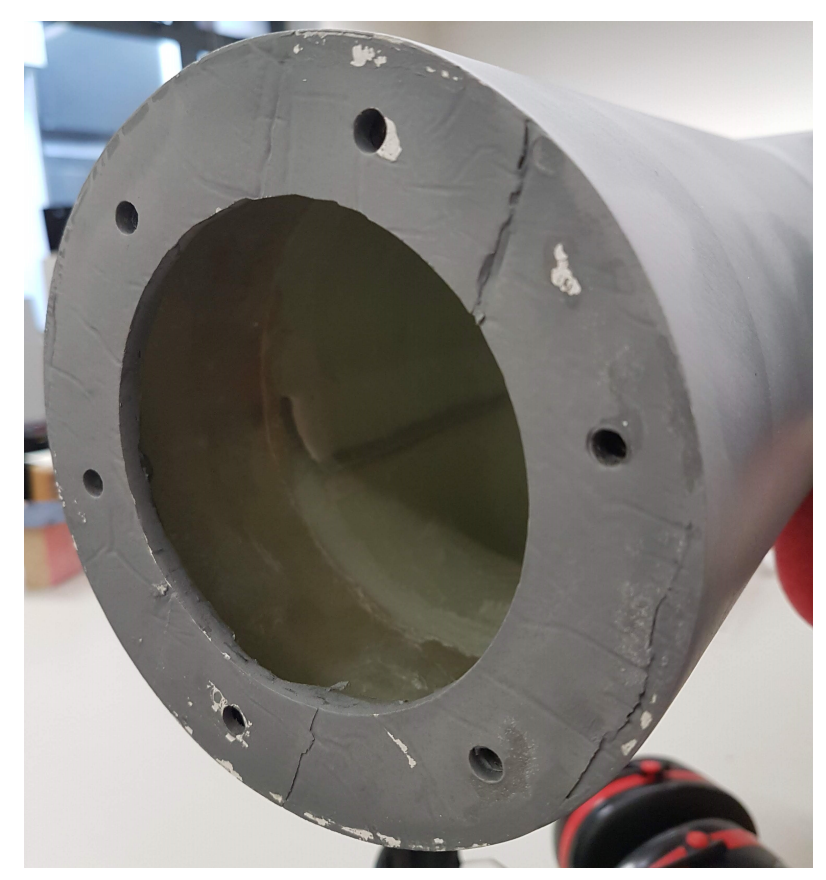

Figure 9.8: One of the motor mounts with an irregular sealing surface caused by the manufacturing process.

To allow for testing with these manufacturing faults, the O-ring was replaced with a liquid gasket compound. The gasket compound conforms much more than the O-ring can, allowing for a potentially better seal. The gasket compound is also used to seal over the heads of the mount bolts to prevent water ingress through the threads. To prevent water damage to the internal components during the water-ingress and buoyancy trials, they are replaced with steel weights of similar mass.

The buoyancy and water-ingress tests were performed in a swimming pool with a water temperature $25^{\circ} \mathrm{C}$. HADES was able to float in the pool with steel weights added to increase the chassis weight to $40 \mathrm{~kg}$; $8 \mathrm{~kg}$ more than the actual weight. Figure 9.9 shows HADES floating in the buoyancy tests.

HADES was floated for 10 mins before the chassis was opened and inspected for water ingress. No obvious leaks were found. To meet the IP68 


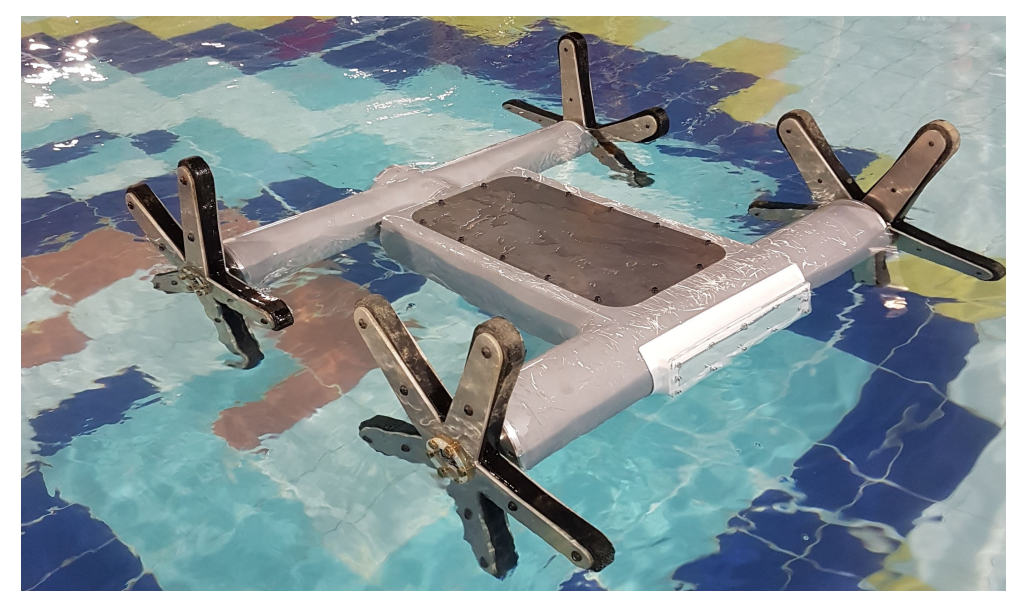

Figure 9.9: HADES floating at the surface for the buoyancy tests.

standard, HADES must be able to withstand submersion in water up to $1 \mathrm{~m}$ deep. As HADES is buoyant this should never occur; however submersion is still possible if HADES falls into water or becomes trapped under falling debris. To test for water submersion, HADES is held $0.8 \mathrm{~m}$ (the maximum depth of the pool) underwater for 5 mins. Figure 9.10 shows HADES being held underwater for the submersion testing.

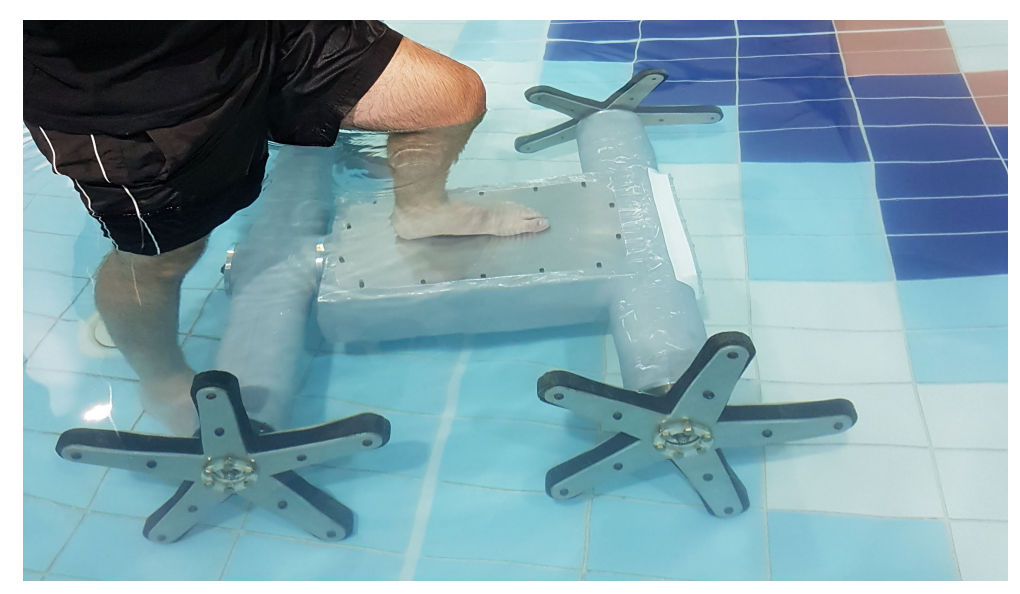

Figure 9.10: HADES being held underwater for the submersion testing of the IP68 standard.

After about $120 \mathrm{~s}$ of submersion, a stream of bubbles could be seen coming 
from the front left motor mount, indicating a leak. The bubbles were appearing from between the drive system and the chassis. There were no other indications of leaks. After 5 mins HADES was removed from the water and the top access hatch was opened for inspection. There was approximately $800 \mathrm{ml}$ of water inside the chassis, indicating a leak with a flow rate of approximately $160 \mathrm{ml} / \mathrm{min}$.

This means that HADES does not meet the IP68 standard. It is expected that the reason for failure was manufacturing faults as the leak appeared to originate from the motor mounts. It is important to note that as the internal components were not inside the chassis at the time of testing; the positive pressure system was not installed. The positive pressure system could potentially reduce the flow rate of the leak as the positive air pressure could prevent water ingress. As another revision of the chassis is needed to install the communication node dispenser mounts, the manufacturing faults could be remedied by an improved manufacturing process.

\subsubsection{Thermal Management}

Fibreglass is a thermal insulator, this prevents the internal components from dissipating heat through the chassis. To prevent damage to the internal component, the chassis temperature should never be greater than $50{ }^{\circ} \mathrm{C}$. HADES has numerous systems for monitoring the temperatures of key system components. After 40 mins of continuous locomotion testing with all the systems active (excluding the pressure system), the temperature of these components was logged. Table 9.7 shows the temperature of the key system components.

The hottest temperature in the system was the CPU in the Intel NUC, this reached a maximum of $76^{\circ} \mathrm{C}$. This is significantly higher than the maximum specification. To validate this, the NUC was run in open air $\left(25^{\circ} \mathrm{C}\right)$ tests for $30 \mathrm{~min}$ and during these tests the NUC CPU reached $73{ }^{\circ} \mathrm{C}$. As the air tem- 
Table 9.7: Key system temperatures of HADES after 30 mins of continuous operation.

\begin{tabular}{|l|l|l|l|}
\hline Sensor & Temperature $\left({ }^{\circ} \mathbf{C}\right)$ & Sensor & Temperature $\left({ }^{\circ} \mathbf{C}\right)$ \\
\hline Chassis Air & 28 & Main PCB & 27 \\
\hline Average ESC & 34 & Power PCB & 33 \\
\hline Intel NUC chassis & 42 & Intel NUC CPU & 76 \\
\hline
\end{tabular}

perature in the chassis after 30 mins was $28{ }^{\circ} \mathrm{C}$, it is expected that the NUC is designed to run at this temperature.

To validate the temperature sensing system, a thermal camera was used to inspect the chassis after 30 mins of testing. Figure 9.11a shows the thermal image of the opened chassis after 30 mins of operation. The hottest identifiable component was the power inductor for the $12 \mathrm{~V}$ regulator (Figure 9.11b). Based on the thermal imagery this was operating at $40{ }^{\circ} \mathrm{C}$, which is below the specification.

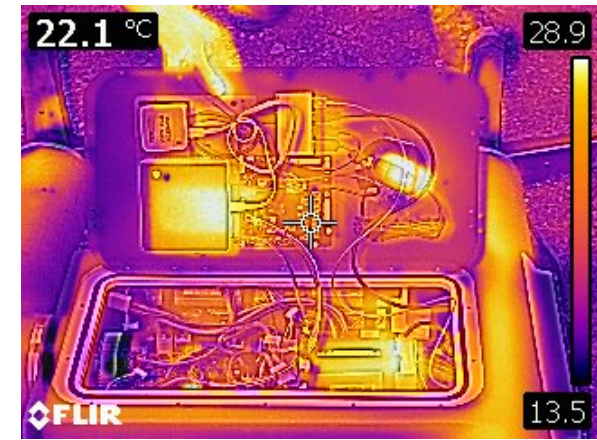

(a) A thermal image of the two main access panels of HADES opened for inspection.

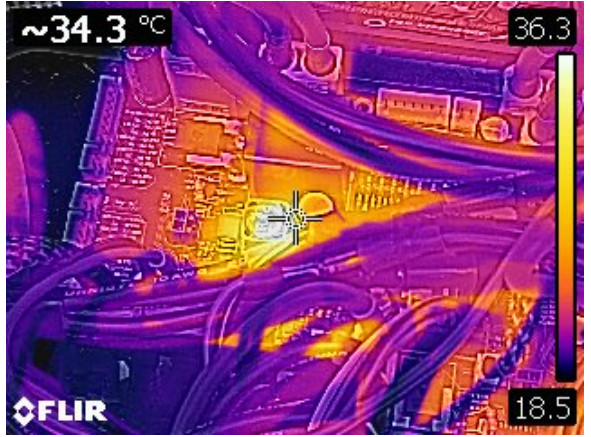

(b) A close up thermal image of the power inductor for the $12 \mathrm{~V}$ regulator.

Figure 9.11: Thermal images of the chassis internals after 30 mins of continuous operation.

During the thermal tests, all of the system temperatures measured remained 
below the required maximum of $50{ }^{\circ} \mathrm{C}$. The tests were completed with the acrylic test plates which are considerably less thermally conductive than the proposed aluminium plates. This suggests that aluminium heat panels might not be necessary, reducing the weight and cost of HADES. An aluminium plate would still need to be used for supporting the rear arm actuation system, as this could potentially break the acrylic plate when under load.

The Intel NUC's CPU produced the highest temperature gradient. If HADES' internal temperature needed to be reduced, the CPU's heat-sink could be replaced. A custom heat-sink design could connect to the external chassis via a heat-transfer device, such as a heat pipe. The external chassis would then itself become the heat-sink, and heat-sink fins could be added if additional surface area is required for increased cooling capacity. A heat-transfer device could also be used to cool the ESCs if acrylic panels are used to replace the proposed aluminium panels.

\subsection{Summary}

HADES was able to navigate through the majority of the locomotion tests successfully. The tests that HADES failed were due to the Whegs not having enough traction or the chassis bottoming out. At higher speeds, the Whegs also caused a large amount of vertical vibration. However, as discussed in Section 4.1 a large diameter Wheg and a six-spoke Wheg have already been designed and could not be manufactured due to the lack of material. These will increase the ground clearance and reduce the vertical vibrations.

During the locomotion testing, HADES was able to function with reduced locomotion power; the drive systems always had enough power and did not overheat. Based on the current usage during the locomotion testing, HADES should be able to travel at cursing speed $\left(1.4 \mathrm{~ms}^{-1}\right)$ for up to 6 hours, this ex- 
ceeds the specification by 2 hours. The rear arm pivot allowed the rear Whegs to gain traction when the front Whegs were off the ground. However, significant galling occurred which could be mitigated by implementing the proposed bronze and steel design. The chassis was able to float and protect against water ingress while on the surface of the water. However, when submerged the chassis leaked which means the IP68 standard was not met. The leaks were due to manufacturing faults which could be remedied by an improved manufacturing process when the node dispenser is integrated.

HADES reported operating well under the $50{ }^{\circ} \mathrm{C}$ thermal limits which was confirmed by thermal camera IR imaging. This was completed with acrylic plates, with the option to improve the thermal performance of HADES by utilising heat pipes and aluminium heat panels. 


\section{Chapter 10}

\section{Conclusion and Future Work}

This chapter summarises the development of the HADES (V2) robot. It also outlines future work that could be conducted to further improve the capabilities of HADES, and concludes with a summary of HADES' capabilities and the milestones that were achieved in this work.

\subsection{Review}

A new robot platform, inspired by previous designs, and especially HADES V1 has been produced to a prototype stage and has been named HADES V2 due to the similarity in chassis shape. A lightweight and robust fibreglass chassis was designed, manufactured and populated for HADES V2. The chassis houses and mounts the internal and external mechanical and electronic components of HADES' subsystems. A pivot system was implemented that allows the rear arm to adapt to the terrain HADES encounters. A system which consists of a motor, gearbox and pulley, was developed to allow the rear arm pivot to be actuated if necessary, which further improves HADES' terrain-traversing capabilities. 
A bespoke steel reinforcement was bonded into the chassis to support the loads from the pivot and drive systems. A re-mountable windscreen system was developed that allows the windscreen material to be easily replaced if it becomes damaged. All of the subsystems are sealed to the chassis with O-rings and lip-seals to prevent the ingress of dust and water. The sealing also allows the use of a static positive pressure gas system that is designed to meet the A/NZ60079.11 standard, allowing HADES to operate in an explosive atmosphere.

For a simple, low cost and reliable locomotion method, a spoked-wheel design was implemented. The spoked-wheels (Whegs) allow HADES to climb over complex and uneven terrain. The Whegs are driven with a 64:1 gearbox and brushless DC motor. To support the high-torque loads from the Whegs and to seal the rotating drive shafts, a bearing block and gearbox mounting system was developed. A bespoke taper lock system was designed to allow the Whegs to be easily mounted to the drive shafts.

Various sensors for navigation and assessment of a post-disaster mine were implemented into an environmental sensor suite for HADES. This can sense seven of the eight common gasses in an underground mine and can measure the atmospheric pressure and temperature in the mine. Each of the gas sensors has a bespoke analogue front end to ensure correct operation of the sensor and to integrate with the other subsystems. The external sensors are protected with an intrinsic safety barrier to meet the A/NZ60079.11 standard. The internal sensor system measures the internal chassis pressure, temperature, oxygen concentration and humidity.

The sensor system includes two IMUs that, when combined with multiple embedded filters, can provide pose and translation information to the operator. A camera system was implemented that uses depth, IR and thermal imaging to assist the operator in navigating through the underground mine, looking for survivors and assessing the condition of the mine for later rescue efforts. An 
LED lighting system was implemented to allow the camera system to obtain high-quality imagery.

The high-level controller was split into multiple independent nodes. Each of these nodes has a critical system function. A node manages the video system and allows the operator to select between the different camera inputs with a bespoke multiplexer. The video node uses Theora image compression to transmit the video stream to the communication system. The hades_error_management node implements basic error handling for the ROS control and the embedded system. The node achieves this by using a set of standardised error codes. HADES can be operated with an $\mathrm{Xbox}^{\mathrm{TM}}$ controller that has the most common robot functions mapped, allowing easy and intuitive operator control. To enable the operator to view telemetry from the robot and update the robot parameters a proof-of-concept GUI was developed that can be accessed using a laptop.

HADES is powered from six Li-Po batteries. A bespoke battery management system protects each of the batteries. The power from the batteries is routed through a series of regulators with dual redundancy and distributed to the system through power management switches. When travelling at a constant cruising speed of $1.4 \mathrm{~ms}^{-1}$ HADES will be able to operate for 6 hours and cover a distance of up to $25 \mathrm{~km}$. In a real-life use case, the operating life of HADES is likely to be closer to 9 hours due to the intermittent use of the drive systems.

\subsection{Future Work}

As discussed in Chapter 2, underground mines are wet, muddy places. Therefore, HADES should meet the IP68 ingress protection standard. However, during testing, HADES failed to meet this standard. It is suspected that this was 
due to defects in the manufacturing process. Possible solutions include repairing the manufacturing defects after manufacture by applying sealant to the defect joints in the chassis. Alternatively, the chassis manufacturing process could be improved to ensure water tightness in a future revision of the chassis.

Section 3.5 proposed an initial design for a sensor pod with 3D printed prototypes. To fully seal and function correctly, the 3D printed parts would need to be machined. The sensor pod design is optimised to be 3D printed in ABS plastic which would not directly translate onto a machining process. Therefore, the design would need to be adapted so that it is suitable for the manufacturing process and material used.

Section 3.1.5 proposed that once the design was completed, the acrylic access panels would be machined from aluminium plate to improve thermal dissipation. However, during testing with acrylic panels, the chassis did not exceed the $50^{\circ} \mathrm{C}$ maximum temperature specification. This indicates that the aluminium plates might not be necessary which would reduce the weight and cost of the chassis. Further investigation would be required to ensure that an acrylic plate has the strength, and thermal properties to ensure the chassis can function as intended.

For the initial testing of HADES, control and telemetry data were sent over an 802.11 WiFi network. WiFi is not suited for deployment in an underground mine due to its limited range and poor propagation. The communication system and node dispensing system developed in parallel to this project would need to be integrated into the chassis and software system. As the fibreglass manufacturer still has the moulds for the chassis, additional features such as the communication system node dispenser could be easily added. If a chassis is produced for mounting the communication nodes, the defects in the manufacturing process that caused water tightness issues could also be resolved.

The Whegs developed in Section 4.1 performed well over most of the terrain types. However, at high speeds, the Whegs caused large vertical movement 
oscillations. The Whegs also sunk into the soft gravel terrain type. Further investigation into the width, tip shape and diameter of the Whegs is required to resolve these issues.

Chapter 7 introduced a bespoke error handling system that spreads across the embedded and ROS control systems in HADES. While the system handles the majority of faults discovered in testing, further investigation is needed to ensure that the software system is robust and can handle the majority of faults. To further increase the reliability and error handling ability of the software system, an investigation into ROS2 should be undertaken. ROS2 aims to improve upon the reliability and security of ROS and is designed for use in industrial robots where safety is a significant concern. The software written for HADES should integrate into ROS2 with minimal effort.

The communication system only has enough bandwidth for a single video stream. This means the operator has to select between the video streams manually to thoroughly survey the mine. An image recognition system running on HADES could identify salient points and alert the operator. This could prevent the operator from driving past a critical feature inside the mine.

HADES has been implemented to allow an unskilled operator to navigate through an underground mine. This means that several crucial software systems perform a self-saving feature, where they would arrest any fault before it can cause damage (e.g. the battery management system). Future systems could be implemented to expand the self-saving functionality. The vision and gas detection systems being a notable example to explore in the first instance.

A prototype GUI was developed for controlling and viewing the status of HADES' elementary functions. Further develoapment of this GUI is required to extend it to cover all aspects of robot function. Additionally, user studies should be undertaken to ensure the GUI is fit for purpose.

The aluminium rear arm pivot performed poorly in the locomotion tests. A 
bronze and steel design was proposed. If implemented, it would significantly extend the operational life of the pivot assembly. This design will need to be explored further, with the bronze and steel pivot design implemented and tested.

\subsection{Summary}

HADES is able to successfully navigate through the terrain expected in an underground mine environment. The chassis is sealed against water jets and is buoyant allowing it to survive in an underground mine (although the chassis is not yet fully IP68 compliant). HADES is designed to meet the A/NZ60079.11 and A/NZ60079.29 standards, allowing HADES to operate safely in an explosive atmosphere.

HADES implements a complex set of atmospheric and visual sensors to aid the operator in navigating through the underground mine. An array of electrochemical and NDIR sensors allow the operator to ascertain the toxicity and explosively of the underground mine for a potential human rescue attempt. The electronics system design is such that each of the subsystems could fail and not affect the complete function of HADES. The embedded software system extends the independent system design and provides an interface for the operator to control all aspects of HADES.

HADES can operate for 6 hours on a single battery charge with an anticipated operating range of $25 \mathrm{~km}$. The LiPo batteries are monitored and protected by a bespoke battery management system. Measuring $780 \times 800 \times 400 \mathrm{~mm}$, it can move through small environments and will provide useful information to rescue teams, potentially saving lives. To conclude, the primary objectives of the thesis and the design specifications for HADES have mostly all been met or exceeded. 


\section{Bibliography}

[1] Hazards of Nitrogen Asphyxiation. Safety bulletin, U.S. Chemical Safety and Hazard Investigation Board, Wshington DC, USA, 2003.

[2] Ventilation in Underground Mines and Tunnels. Standard, Worksafe New Zealand, Wellington, New Zealand, Feb. 2014.

[3] ADAM, J. New correlations between electrical current and temperature rise in pcb traces. In Semiconductor Thermal Measurement and Management Symposium, 2004. Twentieth Annual IEEE (2004), IEEE, pp. 292-299.

[4] Alegro Microsystems. Fully Integrated, Hall Effect-Based Linear Current Sensor IC with 2.1 kVRMS Isolation and a Low-Resistance Current Conductor, 12 2018. Rev. 17.

[5] Alphasense. SO2-A4 Sulfur Dioxide Sensor, 8 2006. Rev. 3.

[6] Alphasense. H2S-A4 Hydrogen Sulfide Sensor, 8 2008. Rev. 3.

[7] Alphasense. CO-A4 Carbon Monoxide Sensor, 8 2010. Rev. 1.

[8] Alphasense Technologies. Designing a Potentiostatic Circuit, 32009. Rev. 0.3.

[9] Analog Devices. Micropower RRIO Operational Amplifiers, 9 2011. Rev. 5.0. 
[10] AnAlog Devices. Low voltage, precision centigrade temperature sensors, 5 2015. Rev. 9.0.

[11] Explosive atmospheres - Equipment protection by intrinsic safety 'i'. Standard, Standards New Zealand, Wellington, New Zealand, Sept. 2011.

[12] Explosive atmospheres - Part 29.2: Gas detectors - Selection, installation, use and maintenance of detectors for flammable gases and oxygen. Standard, Standards New Zealand, Wellington, New Zealand, Apr. 2016.

[13] BABIARZ, P. S. Intrinsic safety circuit design.

[14] Bassily, D., Georgoulas, C., Guettler, J., Linner, T., And Bock, T. Intuitive and adaptive robotic arm manipulation using the leap motion controller. In ISR/robotik 2014; 41st international symposium on robotics; proceedings of (2014), VDE, pp. 1-7.

[15] BLACK, J. R. Electromigration failure modes in aluminum metallization for semiconductor devices. Proceedings of the IEEE 57, 9 (1969), 1587-1594.

[16] BuchmanN, I. How to prolong lithium-based batteries. Batteries in a Portable World 15 (2003), 2-3.

[17] Burke, J. L., Murphy, R. R., Coovert, M. D., And Riddle, D. L. Moonlight in miami: Field study of human-robot interaction in the context of an urban search and rescue disaster response training exercise. HumanComputer Interaction 19, 1-2 (2004), 85-116.

[18] Cao, W., Mecrow, B. C., Atkinson, G. J., Bennett, J. W., And AtKinSON, D. J. Overview of electric motor technologies used for more electric aircraft (mea). IEEE transactions on industrial electronics 59, 9 (2012), 35233531.

[19] CHANG, W.-Y. The state of charge estimating methods for battery: A review. ISRN Applied Mathematics 2013 (2013). 
[20] CHEN, H., AND CHEN, S. A moving average based filtering system with its application to real-time qrs detection. In Computers in Cardiology, 2003 (2003), IEEE, pp. 585-588.

[21] CHEN, J. Y., HAAS, E. C., AND BARNES, M. J. Human performance issues and user interface design for teleoperated robots. IEEE Transactions on Systems, Man, and Cybernetics, Part C (Applications and Reviews) 37, 6 (2007), 1231-1245.

[22] City Technology. IRceL CO2 Carbon Dioxide Sensor, 3 2017. Rev. 1.

[23] City Technology LtD. 4P-50 CiTipel, 12 2004. Rev. 3.

[24] CORKE, P. Integrating ros and matlab [ros topics]. IEEE Robotics $\mathcal{E} A u-$ tomation Magazine 22, 2 (2015), 18-20.

[25] Creitaru, A., AND Grigore, N. Tribological considerations regarding the functional domain determination of the conical fit assembly. machine design 3, 3 (2011), 199-204.

[26] Curcio, V. Chrysler: the life and times of an automotive genius. Oxford University Press on Demand, 2001.

[27] DAVIS, J. R. Surface engineering for corrosion and wear resistance. ASM international, 2001.

[28] Eisenbarth, T., Gong, Z., Güneysu, T., Heyse, S., Indesteege, S., Kerckhof, S., Koeune, F., Nad, T., Plos, T., Regazzoni, F., et Al. Compact implementation and performance evaluation of block ciphers in attiny devices. In International Conference on Cryptology in Africa (2012), Springer, pp. 172-187.

[29] FAllow, N. Wireless Communication for a Mine Scout Robot. Master's thesis, Victoria University of Wellington, Wellington, NZ, 2017. 
[30] Ferrari, M., Morandin, M., And Bolognani, S. Mild hybrid motorcycles: Choice of the energy storage system. In 2012 IEEE International Energy Conference and Exhibition (ENERGYCON) (2012), IEEE, pp. 997-1002.

[31] FIEL, A., AND WU, T. Mosfet failure modes in the zero-voltage-switched full-bridge switching mode power supply applications. In Applied Power Electronics Conference and Exposition, 2001. APEC 2001. Sixteenth Annual IEEE (2001), vol. 2, IEEE, pp. 1247-1252.

[32] GAbriel, D. L., Meyer, J., AND Du Plessis, F. Brushless dc motor characterisation and selection for a fixed wing uav. In AFRICON, 2011 (2011), IEEE, pp. 1-6.

[33] Garretson, J., Hobart, C., and Salton, J. Gemini-scout mine rescue robot. In AUVSI Unmanned Systems North America Conference (2011), pp. $1385-1399$.

[34] Henan Hanwei Electronics CO. ME2-O2 Gas Sensors Manual, 12018. Rev. 1.0.

[35] HoneyWell. Low Voltage Humdidty sensors, 3 2010. Rev. 1.0.

[36] Honeywell. Basic Board MountPressure Sensors, 10 2014. Rev. 1.0.

[37] IEC 60079 Series Explosive Atmosphere Standards. Standard, International Electrotechnical Commission, Washington DC, USA, Mar. 2015.

[38] KASPRZYCZAK, L., SZWEJKOWSKI, P., AND CADER, M. Robotics in mining exemplified by mobile inspection platform. Mining-Informatics, $\mathrm{Au-}$ tomation and Electrical Engineering 54 (2016).

[39] KASPRZYCZAK, L., TRENCZEK, S., AND CADER, M. Robot for monitoring hazardous environments as a mechatronic product. Journal of Automation Mobile Robotics and Intelligent Systems 6 (2012), 57-64. 
[40] Kasprzyczak, L., TrenczeK, S., and Cader, M. Pneumatic robot for monitoring hazardous environments of coal mines. In Solid State Phenomena (2013), vol. 198, Trans Tech Publ, pp. 120-125.

[41] KoOpman, P., And CHAKRAVARTY, T. Cyclic redundancy code (crc) polynomial selection for embedded networks. In Dependable systems and networks, 2004 international conference on (2004), IEEE, pp. 145-154.

[42] KOU, J. A Study of Sensorless Commutation Methods for Brushless DC Motors. Master's thesis, University of Alberta, Alberta, USA, 2017.

[43] Kuenzer, C., And Stracher, G. B. Geomorphology of coal seam fires. Geomorphology 138, 1 (2012), 209-222.

[44] Kukliński, K., Fischer, K., Marhenke, I., Kirstein, F., Maria, V., Sølvason, D., KRÜGer, N., And SAVArimuthu, T. R. Teleoperation for learning by demonstration: Data glove versus object manipulation for intuitive robot control. In Ultra Modern Telecommunications and Control Systems and Workshops (ICUMT), 2014 6th International Congress on (2014), IEEE, pp. 346-351.

[45] LeE, S., Lemley, T., AND KeOHANE, G. A comparison study of the commutation methods for the three-phase permanent magnet brushless dc motor. Pennsylvania State Univ. Berks Campus (2014), 3-5.

[46] LiVesey, R. G. Flow of gases through tubes and orifices, 1998.

[47] Madgwick, S. O., Harrison, A. J., And Vaidyanathan, R. Estimation of imu and marg orientation using a gradient descent algorithm. In Rehabilitation Robotics (ICORR), 2011 IEEE International Conference on (2011), IEEE, pp. 1-7.

[48] Molyneaux, L. Development of an underground mine scout robot. 
[49] Molyneaux, L., Carnegie, D. A., And Chitty, C. Hades: An underground mine disaster scouting robot. In Safety, Security, and Rescue Robotics (SSRR), 2015 IEEE International Symposium on (2015), IEEE, pp. 1-6.

[50] Murphy, R. R. A decade of rescue robots. In 2012 IEEE/RSJ International Conference on Intelligent Robots and Systems (2012), IEEE, pp. 5448-5449.

[51] Murphy, R. R., Kravitz, J., Stover, S. L., And Shoureshi, R. Mobile robots in mine rescue and recovery. IEEE Robotics $\mathcal{E}$ Automation Magazine 16, 2 (2009).

[52] Novak, P., BABJAK, J., Kot, T., Bobovskỳ, Z., Olivka, P., MoczulSKI, W., TimofiejczuK, A., AdAmczyK, M., GuzMan, B. G., ArmadA, A. G., ET AL. Telerescuer-reconnaissance mobile robot for underground coal mines.

[53] Novák, P., BAbjAK, J., Kot, T., And Moczulski, W. Control system of the mobile robot telerescuer. In Applied Mechanics and Materials (2015), vol. 772, Trans Tech Publ, pp. 466-470.

[54] NovÁK, P., Kot, T., BABjAK, J., KonečNỲ, Z., Moczulski, W., AND RODRIGUEZ LÓPEZ, Á. Implementation of explosion safety regulations in design of a mobile robot for coal mines. Applied Sciences 8, 11 (2018), 2300.

[55] Orosy-Fildes, C., AND Allan, R. W. Psychology of computer use: Xii. videogame play: Human reaction time to visual stimuli. Perceptual and motor skills 69, 1 (1989), 243-247.

[56] PAl, N., SAdhu, P. K., Gupta, R. P., AND PRAsAd, U. Review of led based cap lamps for underground coalmines to improve energy efficiency as compared to other light sources. In 2010 The 2nd International Conference on Computer and Automation Engineering (ICCAE) (2010), vol. 5, IEEE, pp. 675-677.

[57] PANCKhurst, C., Bell, S., AND Henry, D. Royal commission on the pike river coal mine tragedy. Wellington: New Zealand Government (2012). 
[58] PARKER, J. Multiple sensors, voting methods, and target value analysis.

[59] Pettersson, M., And Ölvander, J. Drive train optimization for industrial robots. IEEE Transactions on Robotics 25, 6 (2009), 1419-1424.

[60] Philip Hoermandinger, G. K. Design of a Wireless Node Deployment System for the Hades Robot. Master's thesis, Victoria University of Wellington, Wellington, NZ, 2018.

[61] Pounds, P., Hamel, T., And Mahony, R. Attitude control of rigid body dynamics from biased imu measurements. In Decision and Control, 2007 46th IEEE Conference on (2007), IEEE, pp. 4620-4625.

[62] Quigley, M., Conley, K., Gerkey, B., FAust, J., FoOte, T., Leibs, J., WHEELER, R., AND NG, A. Y. Ros: an open-source robot operating system. In ICRA workshop on open source software (2009), vol. 3, Kobe, Japan, p. 5 .

[63] Ralston, J. C., And Hainsworth, D. W. The numbat: A remotely controlled mine emergency response vehicle. In Field and Service Robotics (1998), Springer, pp. 53-59.

[64] Reddy, A. H., Kalyan, B., And Murthy, C. S. Mine rescue robot system-a review. Procedia Earth and Planetary Science 11 (2015), 457-462.

[65] Renesas Electronics Corporation. Micropower Voltage Reference, 3 2018. Rev. 5.0.

[66] REUSS, H.-C. Extended frame format-a new option of the can protocol. Product Concept \& Application Laboratory Hamburg, FR Germany, HAI/AN 92002 (1992).

[67] Rong, X., SONG, R., SONG, X., AND LI, Y. Mechanism and explosionproof design for a coal mine detection robot. Procedia Engineering 15 (2011), 100-104. 
[68] Rudol, P., AND DOHERTY, P. Human body detection and geolocalization for uav search and rescue missions using color and thermal imagery. In Aerospace Conference, 2008 IEEE (2008), IEEE, pp. 1-8.

[69] SEMicONDUCTORS, D. Understanding and using cyclic redundancy checks with dallas semiconductor ibutton products. Tech. rep., Technical Report App Note 27, Dallas Semiconductors, 1996. http:/ /www. dalsemi. com/DocControl/PDFs/appindex. html.

[70] Shigley, J. E. Mechanical engineering design.

[71] TAN, A., ZHANG, T., AND WU, S. Pressure and density of air in mines.

[72] TE Connectivity Measurement Specialties. COMPACT INDUSTRIAL PRESSURE TRANSDUCER, 7 2018. Rev. 2.0.

[73] Texas Instruments. AUtOSWITCHING POWER MUX, 3 2010. Rev. 7.

[74] Texas Instruments. Precision Adjustable Current-Limited PowerDistribution Switches, 12 2016. Rev. 3.

[75] TeXAs Instruments. Switched Capacitor Inverter, 2 2017. Rev. 1.

[76] Ulu, C., El-Kahlout, Y., I, H., O, C., E.U., G., And D.M., B. Design of an inspection robot for search and rescue operations in mines. vol. 3.

[77] VAnguaRD, A. Bombtec defender rov. Retrieved March 1 (2007), 2007.

[78] WU, J. Development of a robotic wireless network for underground mine rescue.

[79] Zientara, M., JakubczyK, D., Kolwas, K., And Kolwas, M. Temperature dependence of the evaporation coefficient of water in air and nitrogen under atmospheric pressure: Study in water droplets. The Journal of Physical Chemistry A 112, 23 (2008), 5152-5158. 